\title{
Aziridine opening via a phenonium ion enables synthesis of complex phenethylamine derivatives
}

\author{
Hannah M. Holst, Jack T. Floreancig, Casey B. Ritts, and Nicholas J. Race* \\ Department of Chemistry, University of Minnesota Twin Cities, Minneapolis, MN 55455
}

SUPPORTING INFORMATION

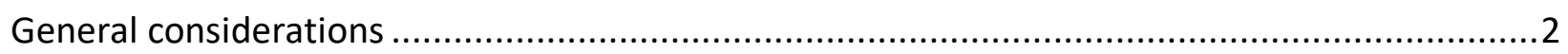

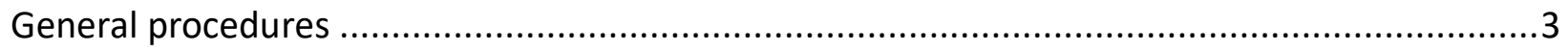

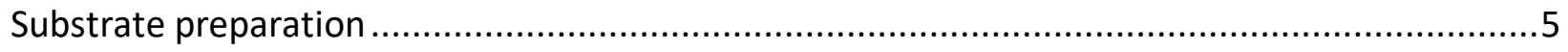

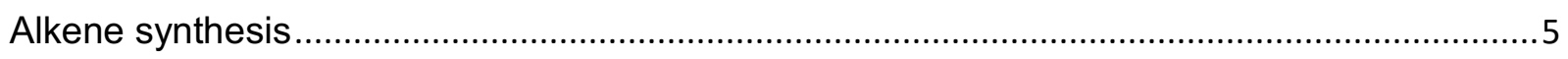

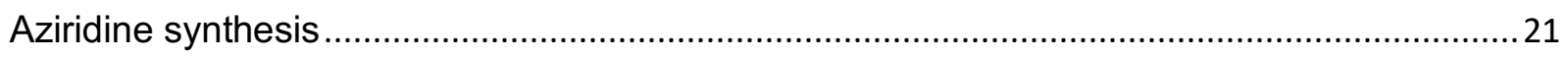

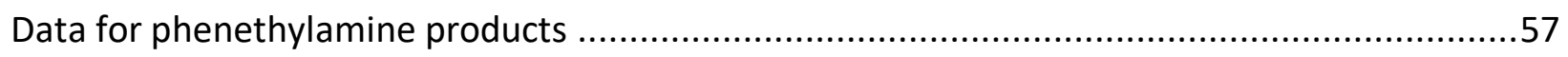

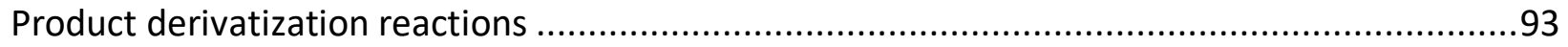

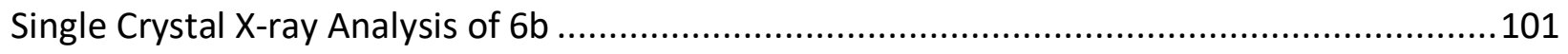

Computational methods, discussion, and results ..................................................... 102 


\section{General considerations}

All reactions were performed using oven-dried or flame-dried glassware equipped with a magnetic stir bar under an atmosphere of argon unless otherwise noted. All reagents were purchased from commercial suppliers and used without further purification. Dichloromethane, tetrahydrofuran, toluene, and acetonitrile were dried by passage through a column of anhydrous alumina using equipment from Pure Process Technology. ${ }^{1} \mathrm{H}$ NMR spectra were obtained in $\mathrm{CDCl}_{3}$ or $\mathrm{C}_{6} \mathrm{D}_{6}$ at $400 \mathrm{MHz}$ or $500 \mathrm{MHz}$. Chemical shifts are reported in ppm and referenced to the $\mathrm{CHCl}_{3}$ singlet at 7.26 ppm. ${ }^{13} \mathrm{C}\left\{{ }^{1} \mathrm{H}\right\}$ NMR spectra were obtained in $\mathrm{CDCl}_{3}$ or $\mathrm{CD}_{2} \mathrm{Cl}_{2}$ at $101 \mathrm{MHz}$ or $126 \mathrm{MHz}$ and referenced to the center peak of the $\mathrm{CDCl}_{3}$ triplet at $77.00 \mathrm{ppm}$. The abbreviations s, d, t, app. h, $\mathrm{dd}$, ddd, $\mathrm{dt}$, and $\mathrm{m}$ stand for the resonance multiplicities singlet, doublet, triplet, apparent heptet, doublet of doublets, doublet of doublet of doublets, doublet of triplets, and multiplet, respectively. Thin-layer chromatography was performed with EMD silica gel $60 \mathrm{~F}_{254}$ plates eluting with solvents indicated, visualized by a $254 \mathrm{~nm}$ UV lamp and stained with potassium permanganate $\left(\mathrm{KMnO}_{4}\right)$ or phosphomolybdic acid (PMA). Flash chromatography was performed using EM reagent silica 60 (230-400 mesh). Infrared (IR) spectra were performed as a film on $\mathrm{NaCl}$ plates on a Nexus $670 \mathrm{FT}-\mathrm{IR}$ and are reported in $\mathrm{cm}^{-1}$. HRMS data were obtained on a Bruker BioTOF II ESI/TOF-MS and Agilent $7200 \mathrm{GC} /$ QTOF-MS. X-ray crystallographic data collection and structure solution were conducted at the X-Ray Crystallographic Laboratory, 192 Kolthoff Hall, Department of Chemistry, University of Minnesota. All calculations were performed using Pentium computers using the current SHELXTL suite of programs. The Bruker-AXS D8 Venture diffractometer was purchased through a grant from NSF/MRI (\#1229400) and the University of Minnesota. Column chromatography was performed using a Teledyne Isco CombiFlash Rf purification system utilizing normal phase pre-column load cartridges and gold high performance columns. 


\section{General procedures}

\section{General Procedure A: for the synthesis of allylbenzene derivatives}

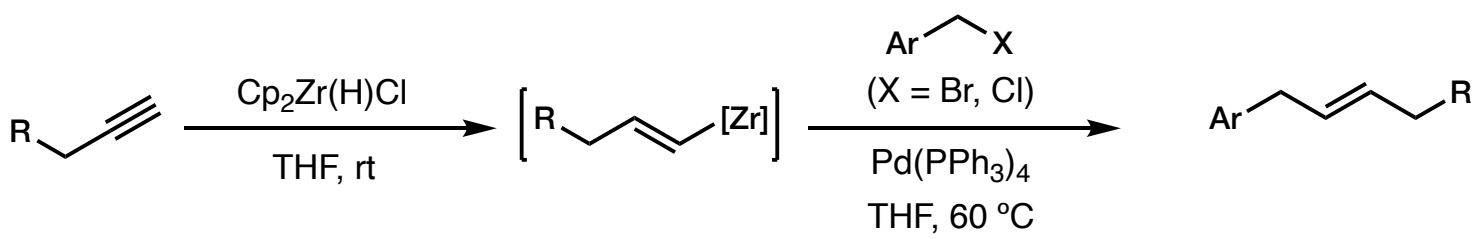

To a solution of Schwartz reagent $(110 \mathrm{mmol} \%)$ in THF $(5 \mathrm{~mL} / \mathrm{mmol}$ alkyne $)$ was added alkyne (100 mol\%) dropwise over five minutes. NB. For large scale reactions $(>10 \mathrm{mmol})$ the addition was performed at $0{ }^{\circ} \mathrm{C}$ due to the mild exotherm that occurs during the addition. The reaction mixture was then stirred at room temperature for one hour, after which time the mixture became clear. The reaction was diluted with THF $(2 \mathrm{~mL} / \mathrm{mmol}$ alkyne) followed by sequential addition of $\mathrm{Pd}\left(\mathrm{PPh}_{3}\right)_{4}(3 \mathrm{~mol} \%)$ and benzyl halide derivative (100 mol\%). The resulting mixture was heated at $60{ }^{\circ} \mathrm{C}$ via a heating block and monitored by TLC analysis. After the reaction was complete, it was cooled to room temperature and concentrated in vacuo. The residue was taken up in hexaneEtOAc $\left(9: 1,10 \mathrm{~mL} / \mathrm{mmol}\right.$ alkyne) and filtered through Celite ${ }^{\circledR}$. The solvent was removed in vacuo and the residue purified by column chromatography to afford the corresponding allylbenzene derivative.

Unless otherwise reported herein, alkenes employed in the synthesis of aziridines have been previously reported. ${ }^{1}$

\section{General Procedure B: for the synthesis of $\mathbf{N}$-Ts aziridines}

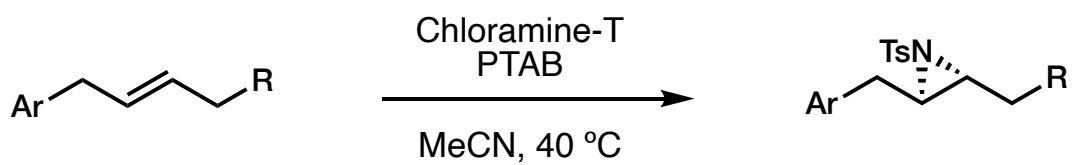

To a solution of alkene (100 mol\%) in MeCN (4 mL/mmol alkene) were added chloramine-T trihydrate $(110 \mathrm{~mol} \%)$ and phenyltrimethylammonium tribromide $(10 \mathrm{~mol} \%)$. The reaction mixture was heated to $40^{\circ} \mathrm{C}$ via a heating block and monitored by TLC analysis. When the reaction was complete, it was cooled to room temperature and concentrated in vacuo. The residue was taken up in hexane-EtOAc $(1: 1,20 \mathrm{~mL} / \mathrm{mmol}$ alkene $)$ and filtered through silica gel. The solvent was removed in vacuo and the residue purified by column chromatography to afford the corresponding $N$-Ts aziridine.

1 Xu, S.; Holst, H. M.; McGuire, S. B.; Race, N. J. J. Am. Chem. Soc. 2020, 142, 8090-8096 
General Procedure C: for the synthesis of $\mathbf{N}$-Ac aziridines

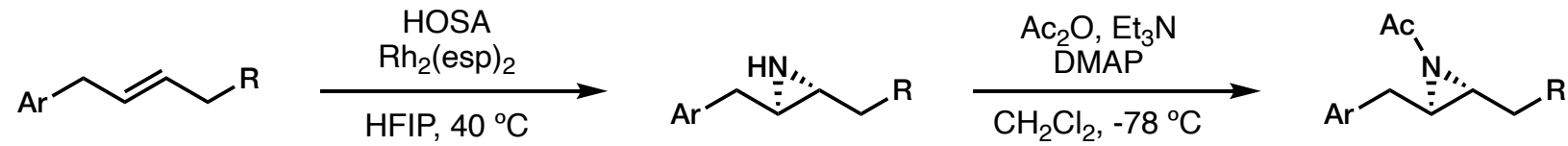

To a solution of alkene (100 mol\%) in HFIP $(2.5 \mathrm{~mL} / \mathrm{mmol})$ was added bis[rhodium( $\alpha, \alpha, \alpha^{\prime}, \alpha^{\prime}-$ tetramethyl-1,3-benzenedipropionic acid)] (2 mol\%), pyridine (120 mol\%), and hydroxylamine-Osulfonic acid $(120 \mathrm{~mol} \%)$. The mixture was heated at $40{ }^{\circ} \mathrm{C}$ via an aluminum heating block until complete consumption of starting material was observed by TLC analysis. Upon completion, the reaction was quenched with saturated aqueous $\mathrm{Na}_{2} \mathrm{CO}_{3}(2 \mathrm{~mL} / \mathrm{mmol}$ alkene) and extracted with dichloromethane ( $3 \times 5 \mathrm{~mL} / \mathrm{mmol}$ alkene). The organic layers were combined and washed with brine ( $3 \times 5 \mathrm{~mL} / \mathrm{mmol}$ alkene), dried with $\mathrm{MgSO}_{4}$, and concentrated in vacuo. The crude material was used without further purification. ${ }^{2}$

To a solution of crude material in $\mathrm{CH}_{2} \mathrm{Cl}_{2}(2.5 \mathrm{~mL} / \mathrm{mmol})$ was added triethylamine $(200 \mathrm{~mol} \%)$ and 4-dimethylaminopyridine $(3 \mathrm{~mol} \%)$. The mixture was cooled to $-78^{\circ} \mathrm{C}$ and acetic anhydride $(110$ mol\%) was added dropwise over five minutes. The reaction was monitored by TLC analysis. Upon completion, the reaction was quenched with water $\left(5 \mathrm{~mL} / \mathrm{mmol}\right.$ alkene) and extracted with $\mathrm{Et}_{2} \mathrm{O}$ ( $3 \times 5 \mathrm{~mL} / \mathrm{mmol}$ alkene). The organic layers were combined and washed with water $(1 \times 5$ $\mathrm{mL} / \mathrm{mmol}$ alkene), saturated aqueous $\mathrm{NaHCO}_{3}(1 \times 5 \mathrm{~mL} / \mathrm{mmol}$ alkene $)$, and brine $(1 \times 5 \mathrm{~mL} / \mathrm{mmol}$ alkene), dried $\left(\mathrm{MgSO}_{4}\right)$, and concentrated in vacuo. The residue was then purified by column chromatography to afford the corresponding acetyl protected aziridine.

\section{General Procedure D: for the formation of $\beta$-phenethylamines from aziridines}

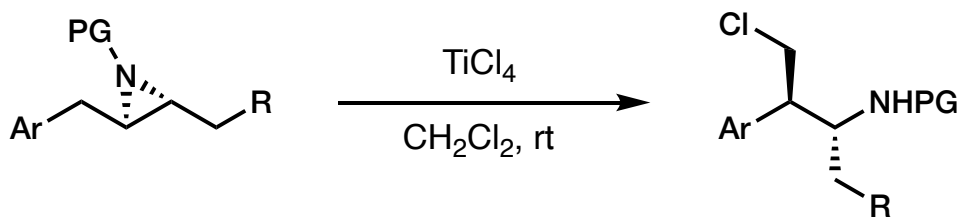

To an oven-dried reaction tube, charged with a stir bar, was added the aziridine derivative (100 mol\%). The tube was sealed with a septum and purged with argon after which anhydrous $\mathrm{CH}_{2} \mathrm{Cl}_{2}$ (0.1 $\mathrm{M}$ total reaction concentration) was added. A freshly prepared solution of $\mathrm{TiCl}_{4}(0.5 \mathrm{M}$ in $\mathrm{CH}_{2} \mathrm{Cl}_{2}, 50 \mathrm{~mol} \%$ ) was added dropwise via syringe over five minutes. The reaction was stirred at room temperature for 10 minutes and quenched with saturated aqueous $\mathrm{NaHCO}_{3}(1 \mathrm{~mL} / \mathrm{mmol}$ aziridine). The solution was filtered through Celite $\AA$, washed with water $(2 \times 10 \mathrm{~mL} / \mathrm{mmol}$ aziridine $)$ and brine $\left(10 \mathrm{~mL} / \mathrm{mmol}\right.$ aziridine), dried with $\mathrm{MgSO}_{4}$, and concentrated in vacuo. The residue was purified by column chromatography to afford the corresponding pure phenethylamine.

\footnotetext{
${ }^{2}$ Ma, Z.; Zhou, Z.; Kürti, L.; Angew. Chem., Int. Ed. 2017, 56, 9886-9890
} 


\section{Substrate preparation}

\section{Alkene synthesis}<smiles>CCC/C=C/Cc1ccc(C)c(C)c1</smiles>

\section{(E)-4-(Hex-2-en-1-yl)-1,2-dimethylbenzene S1}

General Procedure A: 1-Pentyne $(1.1 \mathrm{~mL}, 11.6 \mathrm{mmol})$ and 3,4-dimethylbenzyl bromide $(2.30 \mathrm{~g}$, $11.6 \mathrm{mmol}$ ) were employed. Purification of the residue by column chromatography $(100 \%$ hexanes) afforded the title compound $(1.90 \mathrm{~g}, 87 \%)$ as a colorless oil.

${ }^{1} \mathrm{H}$ NMR $\left(500 \mathrm{MHz}, \mathrm{CDCl}_{3}\right) \delta 7.05(\mathrm{~d}, J=7.6 \mathrm{~Hz}, 1 \mathrm{H}), 6.96(\mathrm{~d}, J=1.9 \mathrm{~Hz}, 1 \mathrm{H}), 6.92$ (dd, $J=7.6$, $1.9 \mathrm{~Hz}, 1 \mathrm{H}), 5.58-5.47(\mathrm{~m}, 2 \mathrm{H}), 3.27(\mathrm{~d}, J=6.1 \mathrm{~Hz}, 2 \mathrm{H}), 2.24(\mathrm{~s}, 3 \mathrm{H}), 2.23(\mathrm{~s}, 3 \mathrm{H}), 2.00$ (tdd, $J=$ 7.4, 6.2, $0.8 \mathrm{~Hz}, 2 \mathrm{H}), 1.40$ (h, $J=7.8 \mathrm{~Hz}, 2 \mathrm{H}), 0.90$ (t, $J=7.4 \mathrm{~Hz}, 3 \mathrm{H})$;

${ }^{13} \mathrm{C}$ NMR $\left(126 \mathrm{MHz}, \mathrm{CDCl}_{3}\right) \delta 138.5,136.4,133.9,131.5,129.8,129.6,129.3,125.8,38.6,34.60$, 22.6, 19.7, 19.3, 13.7;

IR (NaCl, thin film) 2958, 2924, 1504, 1455, 1381, $1021 \mathrm{~cm}^{-1}$;

HRMS $\left(\mathrm{El}^{+}\right) \mathrm{m} / \mathrm{z}$ calcd for $\mathrm{C}_{14} \mathrm{H}_{20}{ }^{+}(\mathrm{M})^{+}:$188.1565, found 188.1555 . 

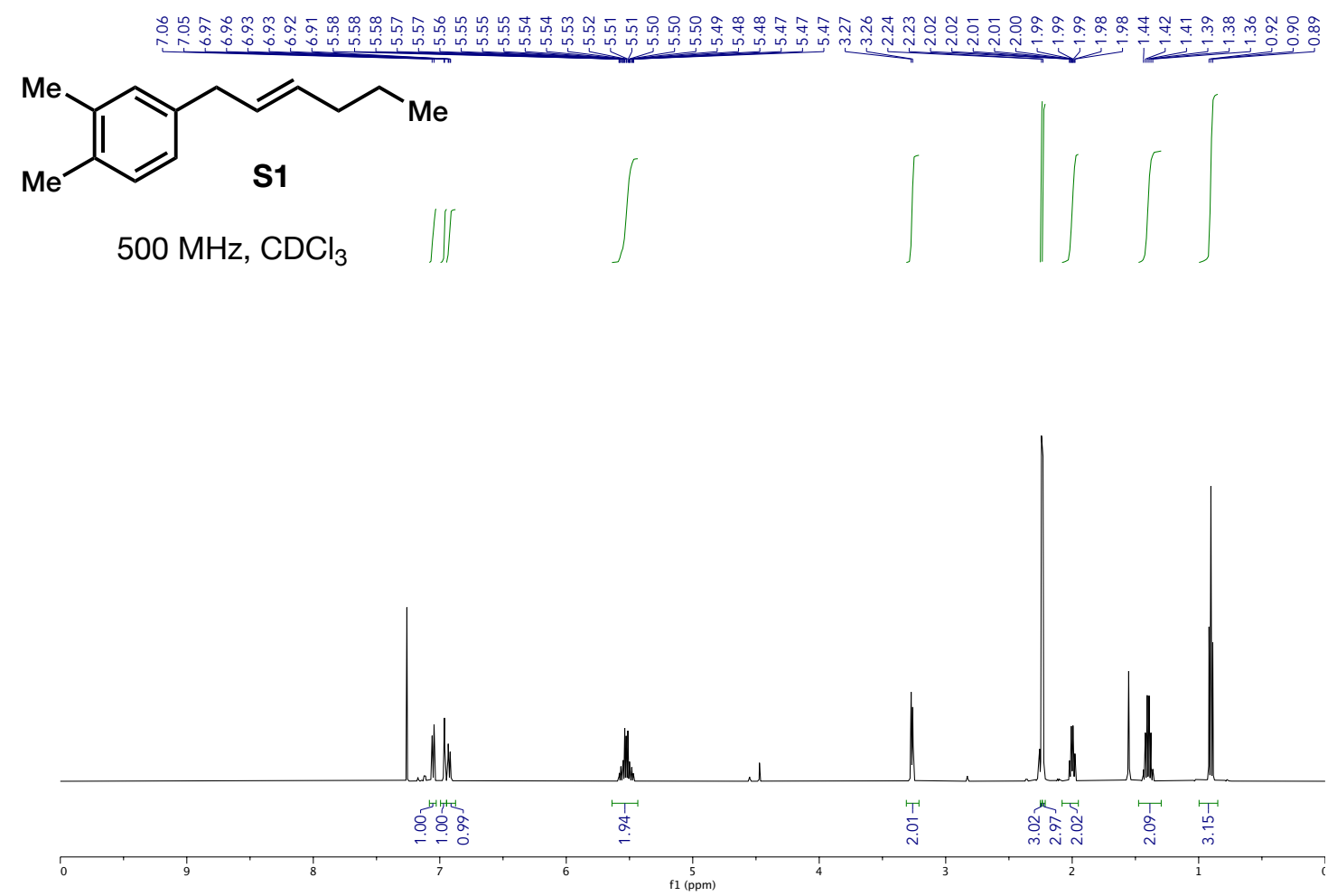<smiles>CCC/C=C/Cc1ccc(C)c(C)c1</smiles>
$126 \mathrm{MHz}, \mathrm{CDCl}_{3}$

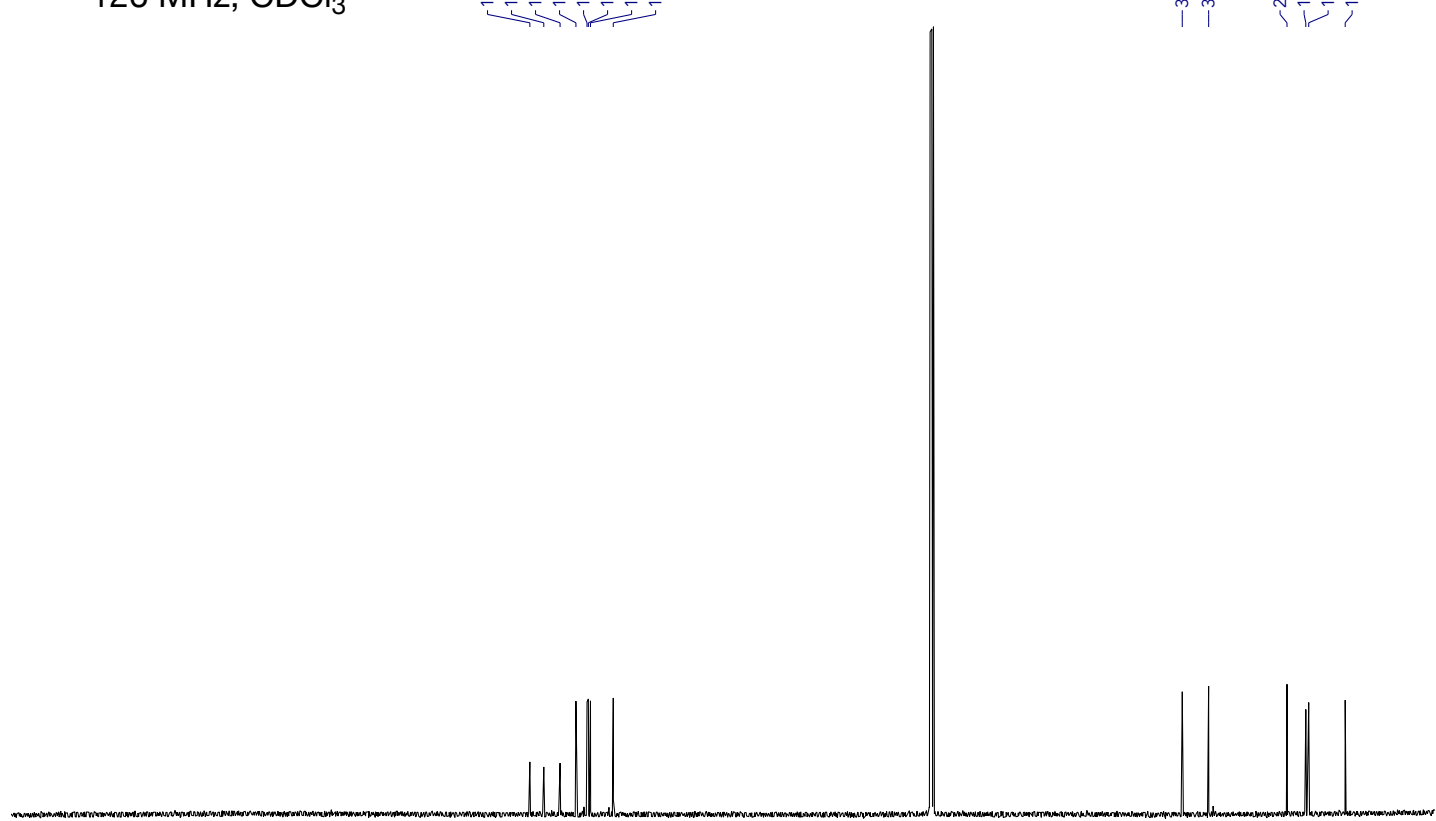

200

180

160

140

120

100

80

60

40

${ }_{20}^{1}+c$ 
<smiles>CCC/C=C/Cc1ccc(OC)c(Br)c1</smiles>

\section{(E)-2-Bromo-4-(hex-2-en-1-yl)-1-methoxybenzene S2}

General Procedure A: 1-Pentyne $(0.91 \mathrm{~mL}, 9.24 \mathrm{mmol})$ and 2-bromo-4-(bromomethyl)-1methoxybenzene $(2.59 \mathrm{~g}, 9.24 \mathrm{mmol})$ were employed. Purification of the residue by column chromatography (20:1, hexanes:EtOAc) afforded the title compound $(2.12 \mathrm{~g}, 85 \%)$ as a colorless oil.

${ }^{1} \mathrm{H}$ NMR $\left(500 \mathrm{MHz}, \mathrm{CDCl}_{3}\right) \delta 7.39(\mathrm{~d}, J=2.1 \mathrm{~Hz}, 1 \mathrm{H}), 7.10(\mathrm{dd}, J=8.4,2.2 \mathrm{~Hz}, 1 \mathrm{H}), 6.84(\mathrm{~d}, J=$ $8.4 \mathrm{~Hz}, 1 \mathrm{H}), 5.59-5.47(\mathrm{~m}, 2 \mathrm{H}), 3.89(\mathrm{~s}, 3 \mathrm{H}), 3.27(\mathrm{~d}, J=4.8 \mathrm{~Hz}, 2 \mathrm{H}), 2.09-1.97(\mathrm{~m}, 2 \mathrm{H}), 1.49-$ $1.37(\mathrm{~m}, 2 \mathrm{H}), 0.93(\mathrm{t}, J=7.4 \mathrm{~Hz}, 3 \mathrm{H})$.

${ }^{13} \mathrm{C}$ NMR $\left(126 \mathrm{MHz}, \mathrm{CDCl}_{3}\right) \delta 154.1,134.8,133.2,132.3,128.5,128.3,111.9,111.5,56.3,37.8$, $34.6,22.6,13.7$;

IR ( $\mathrm{NaCl}$, thin film) 3004, 2927, 1603, 1496, $1438 \mathrm{~cm}^{-1}$;

HRMS (El') $m / z$ calcd for $\mathrm{C}_{13} \mathrm{H}_{17} \mathrm{BrO}^{+}(\mathrm{M})^{+}:$268.0457, found 268.0454. 

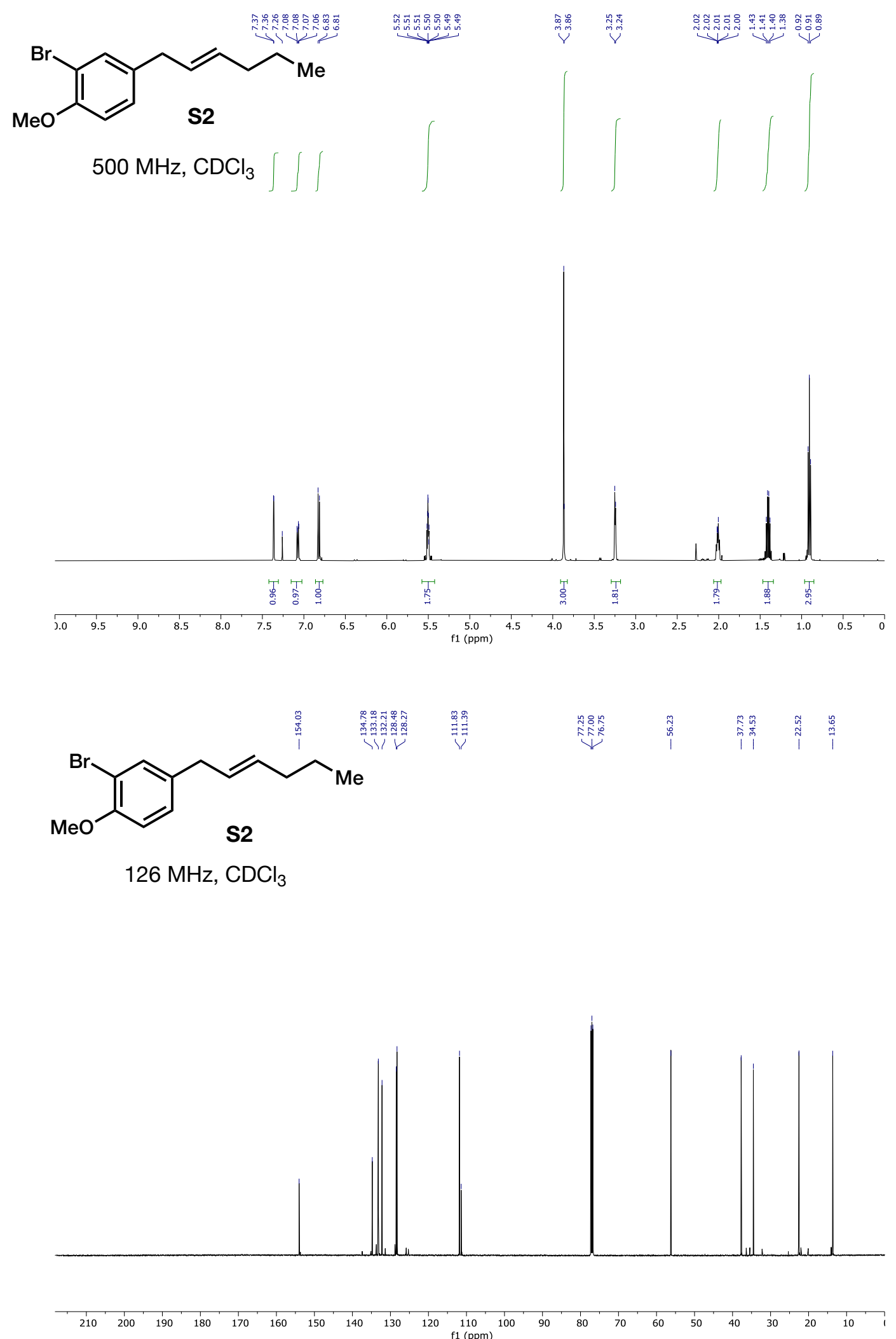
<smiles>CCC/C=C/Cc1ccc(N2CCOCC2)cc1</smiles>

\section{(E)-4-(4-(Hex-2-en-1-yl)phenyl)morpholine S3}

To a solution of $(E)$-1-bromo-4-(hex-2-en-1-yl)benzene $(0.96 \mathrm{~g}, 4.0 \mathrm{mmol})$ in toluene $(13 \mathrm{~mL})$ was added potassium tert-butoxide $(1.35 \mathrm{~g}, 12.0 \mathrm{mmol})$, morpholine $(0.69 \mathrm{~mL}, 8.0 \mathrm{mmol}), \mathrm{Pd}_{2}(\mathrm{dba})_{3}$ $(0.19 \mathrm{~g}, 0.2 \mathrm{mmol})$, and 2,2'-bis-(diphenylphosphino)-1,1'-binaphthyl (0.25 g, $0.4 \mathrm{mmol})$. The mixture was heated to $80{ }^{\circ} \mathrm{C}$ overnight via an aluminum heating block and then concentrated in vacuo. Purification of the residue by column chromatography $\left(5: 1\right.$, hexanes:EtOAc with $\left.1 \% \mathrm{Et}_{3} \mathrm{~N}\right)$ afforded the title compound $(0.58 \mathrm{~g}, 60 \%)$ as a brown oil. ${ }^{3}$

${ }^{1} \mathrm{H}$ NMR $\left(500 \mathrm{MHz}, \mathrm{CDCl}_{3}\right) \delta 7.10(\mathrm{~d}, \mathrm{~J}=8.6 \mathrm{~Hz}, 2 \mathrm{H}), 6.86(\mathrm{~d}, \mathrm{~J}=8.2 \mathrm{~Hz}, 2 \mathrm{H}), 5.58-5.45(\mathrm{~m}, 2 \mathrm{H})$, 3.90-3.84 (m, 4H), 3.26 (d, J = 6.3 Hz, 2H), 3.16-3.10 (m, 4H), 2.04-1.96 (m, 2H), 1.45-1.34 (m, $2 \mathrm{H}), 0.90(\mathrm{t}, \mathrm{J}=7.4 \mathrm{~Hz}, 3 \mathrm{H})$;

${ }^{13} \mathrm{C}$ NMR $\left(126 \mathrm{MHz}, \mathrm{CDCl}_{3}\right) \delta 149.5,132.8,131.5,129.3,129.1,116.0,67.0,49.8,38.1,34.6$, 22.6, 13.7;

IR $\left(\mathrm{NaCl}\right.$, thin film) 2958, 2924, 1514, $1261 \mathrm{~cm}^{-1}$;

HRMS (ESI-TOF ${ }^{+}$) $\mathrm{m} / \mathrm{z}$ calcd for $\mathrm{C}_{16} \mathrm{H}_{23} \mathrm{NOH}(\mathrm{M}+\mathrm{H})^{+}:$: 246.1852, found 246.1862.

3 Larsen, S. B.; Bang-Andersen, B.; Johansen, T. N.; Jørgensen, M. Tetrahedron 2008, 64, 2938-2950. 

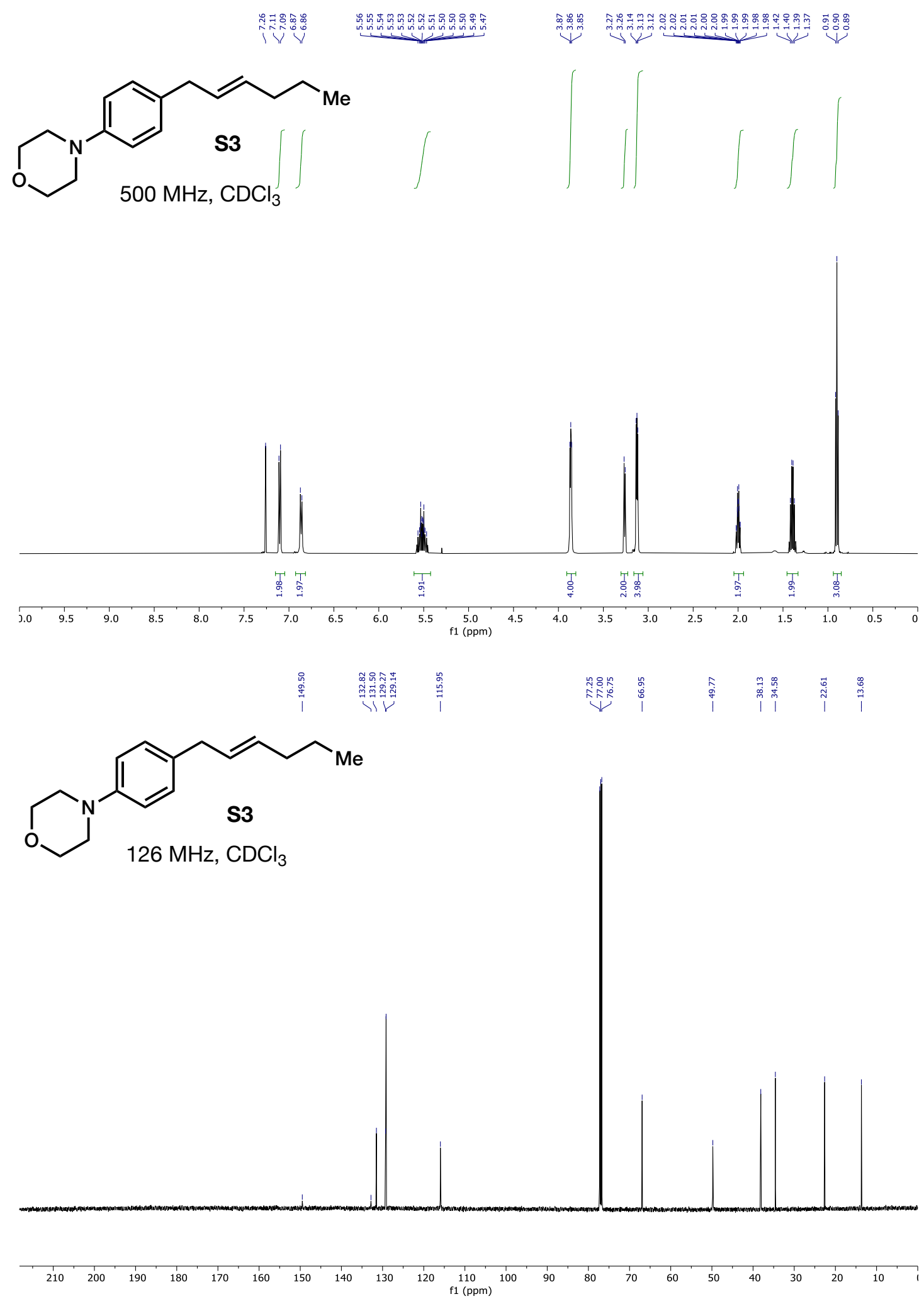


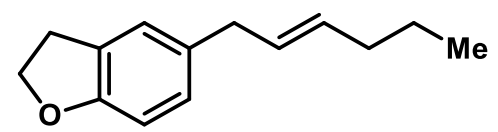

\section{(E)-5-(hex-2-en-1-yI)-2,3-dihydrobenzofuran S4}

General Procedure A: 1-Pentyne (0.95 mL, $9.60 \mathrm{mmol})$ and 5-(bromomethyl)-2,3dihydrobenzofuran $(2.05 \mathrm{~g}, 9.60 \mathrm{mmol})$ were employed. Purification of the residue by column chromatography (10:1, hexanes:EtOAc) afforded the title compound (1.15 g, 59\%) as a colorless oil.

${ }^{1} \mathrm{H}$ NMR $\left(500 \mathrm{MHz}, \mathrm{CDCl}_{3}\right) \delta 7.04(\mathrm{~d}, J=1.9 \mathrm{~Hz}, 1 \mathrm{H}), 6.94(\mathrm{dd}, J=8.2,1.9 \mathrm{~Hz}, 1 \mathrm{H}), 6.73(\mathrm{~d}, J=$ $8.1 \mathrm{~Hz}, 1 \mathrm{H}), 5.62-5.46(\mathrm{~m}, 2 \mathrm{H}), 4.57(\mathrm{t}, J=8.7 \mathrm{~Hz}, 2 \mathrm{H}), 3.28(\mathrm{~d}, J=6.2 \mathrm{~Hz}, 2 \mathrm{H}), 3.20(\mathrm{t}, J=8.6$ $\mathrm{Hz}, 2 \mathrm{H}$ ), 2.07-1.99 (m, 2H), 1.43 (q, $J=7.4 \mathrm{~Hz}, 2 \mathrm{H}), 0.93$ (t, $J=7.4 \mathrm{~Hz}, 3 \mathrm{H})$;

${ }^{13} \mathrm{C}$ NMR $\left(126 \mathrm{MHz}, \mathrm{CDCl}_{3}\right) \delta 158.3,133.1,131.4,129.6,127.8,126.9,124.9,108.9,71.1,38.5$, 34.6, 29.8, 22.6, 13.7;

IR ( $\mathrm{NaCl}$, thin film) 3014, 2838, 1438, 1360, $1242 \mathrm{~cm}^{-1}$;

HRMS $\left(\mathrm{El}^{+}\right) \mathrm{m} / \mathrm{z}$ calcd for $\mathrm{C}_{14} \mathrm{H}_{18} \mathrm{O}^{+}(\mathrm{M})^{+}:$202.1358, found 202.1353. 

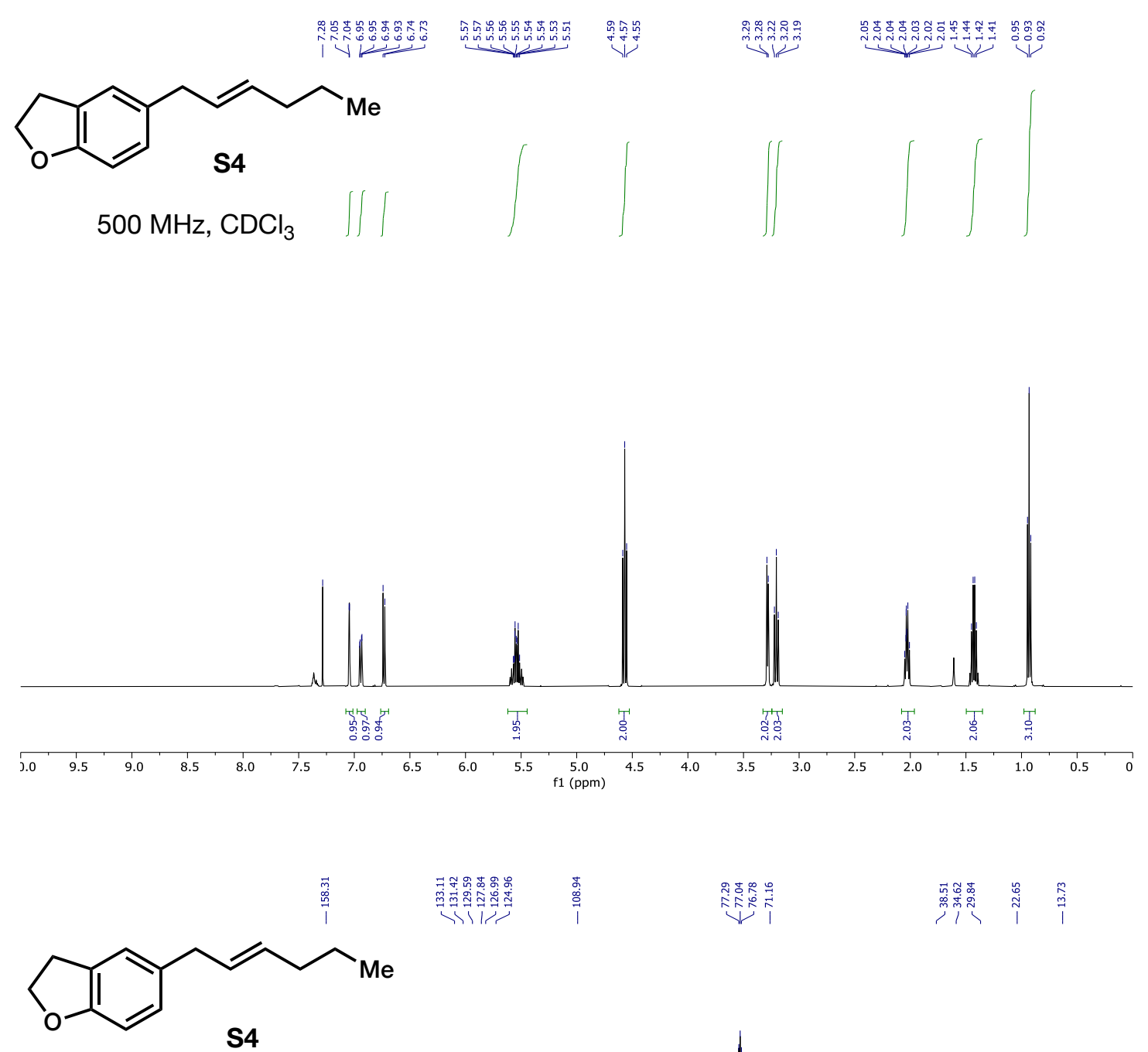

$126 \mathrm{MHz}, \mathrm{CDCl}_{3}$

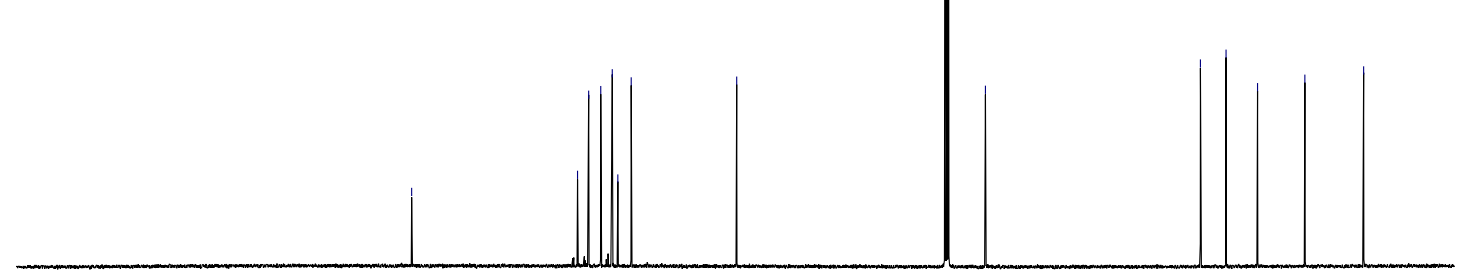




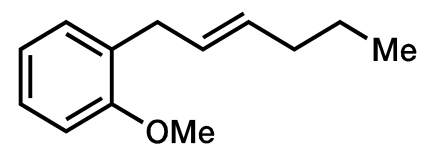

(E)-1-(Hex-2-en-1-yl)-2-methoxybenzene S5

General Procedure A: 1-Pentyne (1.1 mL, $10.9 \mathrm{mmol})$ and 2-methoxybenzyl bromide (2.20 g, 10.9 $\mathrm{mmol}$ ) were employed. Purification of the residue by column chromatography $(100 \%$ hexanes) afforded the title compound $(1.63 \mathrm{~g}, 78 \%)$ as a colorless oil.

${ }^{1} \mathrm{H}$ NMR $\left(500 \mathrm{MHz}, \mathrm{CDCl}_{3}\right) \delta 7.19(\mathrm{td}, J=7.7,1.8 \mathrm{~Hz}, 1 \mathrm{H}), 7.15(\mathrm{dd}, J=7.4,1.8 \mathrm{~Hz}, 1 \mathrm{H}), 6.90$ $(\mathrm{td}, J=7.4,1.1 \mathrm{~Hz}, 1 \mathrm{H}), 6.85(\mathrm{dd}, J=8.1,1.1 \mathrm{~Hz}, 1 \mathrm{H}), 5.59(\mathrm{dtt}, J=15.7,6.5,1.3 \mathrm{~Hz}, 1 \mathrm{H}), 5.48$ (dtt, $J=14.9,6.7,1.3 \mathrm{~Hz}, 1 \mathrm{H}$ ), $3.83(\mathrm{~s}, 3 \mathrm{H}), 3.33(\mathrm{~d}, J=6.6 \mathrm{~Hz}, 2 \mathrm{H}), 2.00$ (tdd, $J=7.7,6.1,1.2$ $\mathrm{Hz}, 2 \mathrm{H}$ ), 1.39 (h, J=7.3 Hz, 2H), 0.90 (t, J=7.4 Hz, 3H);

${ }^{13} \mathrm{C}$ NMR $\left(126 \mathrm{MHz}, \mathrm{CDCl}_{3}\right) \delta 157.2,131.5,129.6,129.5,128.2,127.0,120.4,110.2,55.3,34.7$, 32.9, 22.6, 13.7;

IR ( $\mathrm{NaCl}$, thin film) 2959, 2932, 1600, 1482, 1463, $1243 \mathrm{~cm}^{-1}$;

HRMS $\left(\mathrm{El}^{+}\right) \mathrm{m} / \mathrm{z}$ calcd for $\mathrm{C}_{13} \mathrm{H}_{18} \mathrm{O}^{+}(\mathrm{M})^{+}:$190.1358, found 190.1347 . 


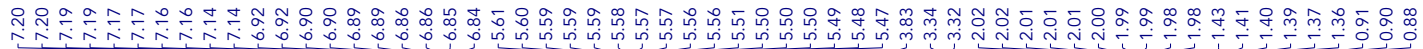

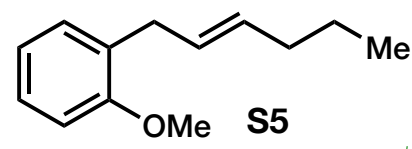

$500 \mathrm{MHz}, \mathrm{CDCl}_{3}$
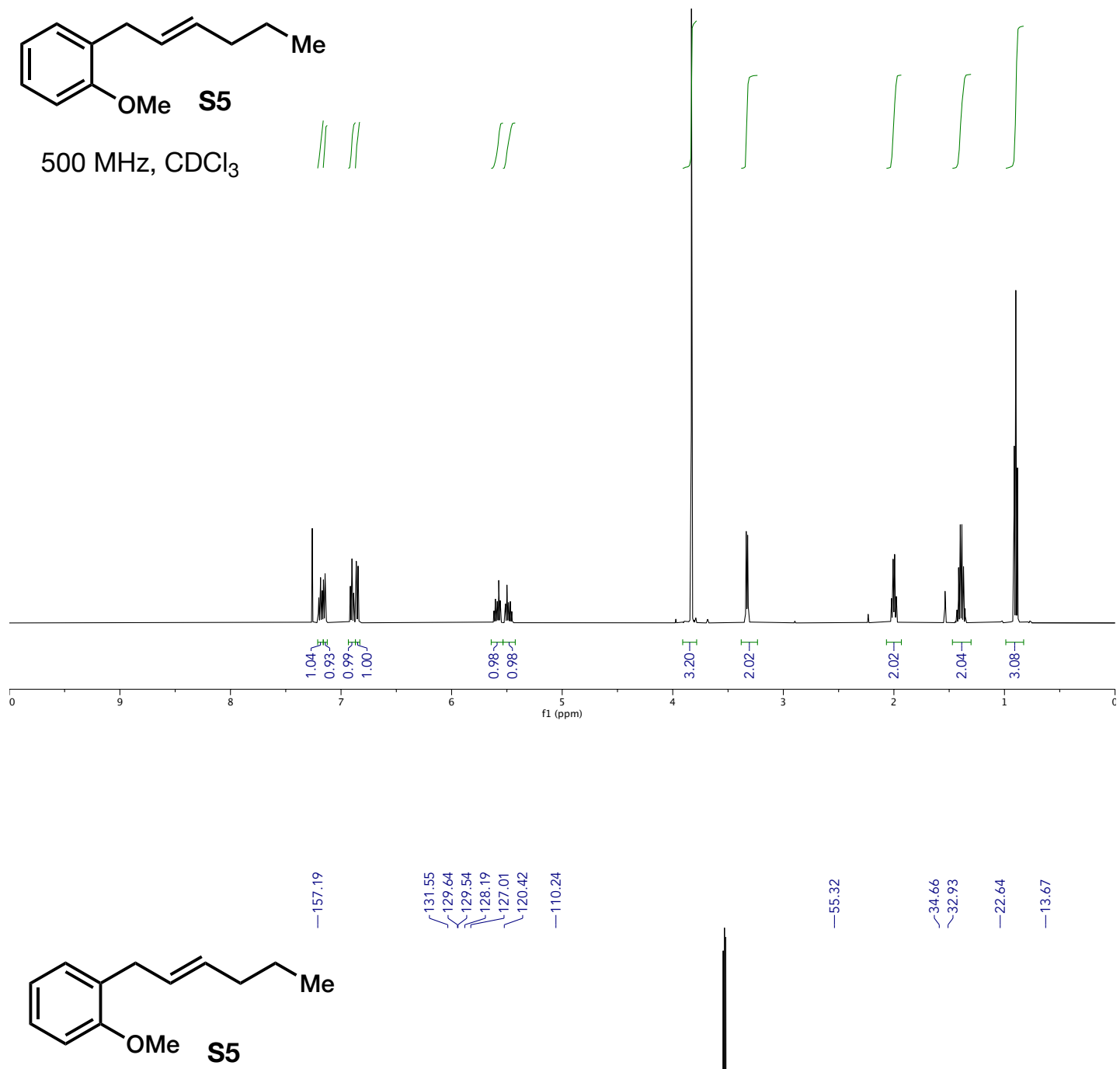

$126 \mathrm{MHz}, \mathrm{CDCl}_{3}$

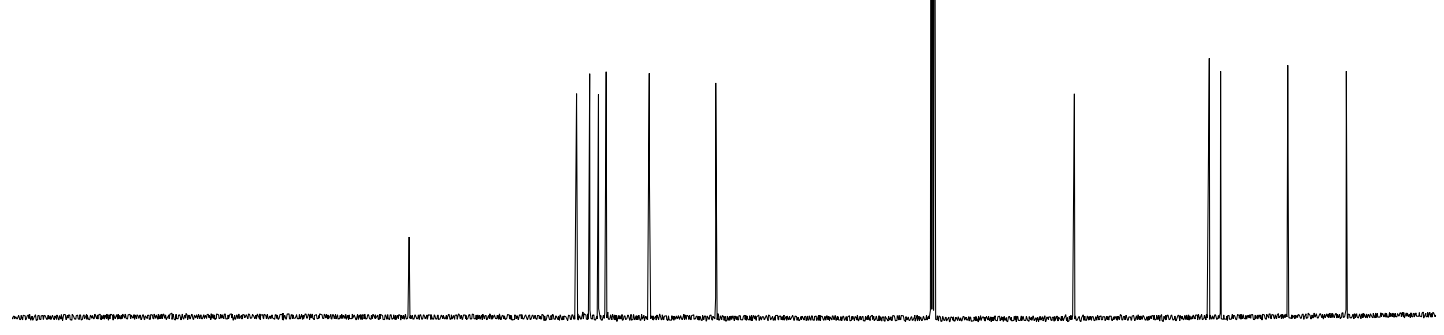

S-14 
<smiles>COc1ccc(C/C=C/CC(C)C)cc1</smiles>

\section{(E)-1-Methoxy-4-(5-methylhex-2-en-1-yl)benzene S6}

General Procedure A: 1-Pentyne $(1.20 \mathrm{~mL}, 12.8 \mathrm{mmol})$ and 4-methoxybenzyl bromide $(2.57 \mathrm{~g}$, $12.8 \mathrm{mmol})$ were employed. Purification of the residue by column chromatography (10:1, hexanes:EtOAc) afforded the title compound $(1.36 \mathrm{~g}, 52 \%)$ as a colorless oil.

${ }^{1} \mathrm{H}$ NMR $\left(500 \mathrm{MHz}, \mathrm{CDCl}_{3}\right) \delta 7.13(\mathrm{~d}, J=8.7 \mathrm{~Hz}, 2 \mathrm{H}), 6.87$ (d, $\left.J=8.6 \mathrm{~Hz}, 2 \mathrm{H}\right), 5.61-5.45(\mathrm{~m}, 2 \mathrm{H})$, $3.82(\mathrm{~s}, 3 \mathrm{H}), 3.31(\mathrm{~d}, J=6.3 \mathrm{~Hz}, 2 \mathrm{H}), 1.98-1.90(\mathrm{~m}, 2 \mathrm{H}), 1.65(\mathrm{dt}, J=13.3,6.7 \mathrm{~Hz}, 1 \mathrm{H}), 0.92(\mathrm{~d}$, $J=6.7 \mathrm{~Hz}, 6 \mathrm{H})$;

${ }^{13} \mathrm{C}$ NMR $\left(126 \mathrm{MHz}, \mathrm{CDCl}_{3}\right) \delta 157.8,133.2,130.5,130.3,129.3,113.8,55.3,41.9,38.2,28.48$, 22.3;

IR ( $\mathrm{NaCl}$, thin film) 3029, 2997, 1584, 1511, $1440 \mathrm{~cm}^{-1}$;

HRMS $\left(\mathrm{El}^{+}\right) \mathrm{m} / \mathrm{z}$ calcd for $\mathrm{C}_{14} \mathrm{H}_{20} \mathrm{O}^{+}(\mathrm{M})^{+}:$: 204.1514, found 204.1491 . 

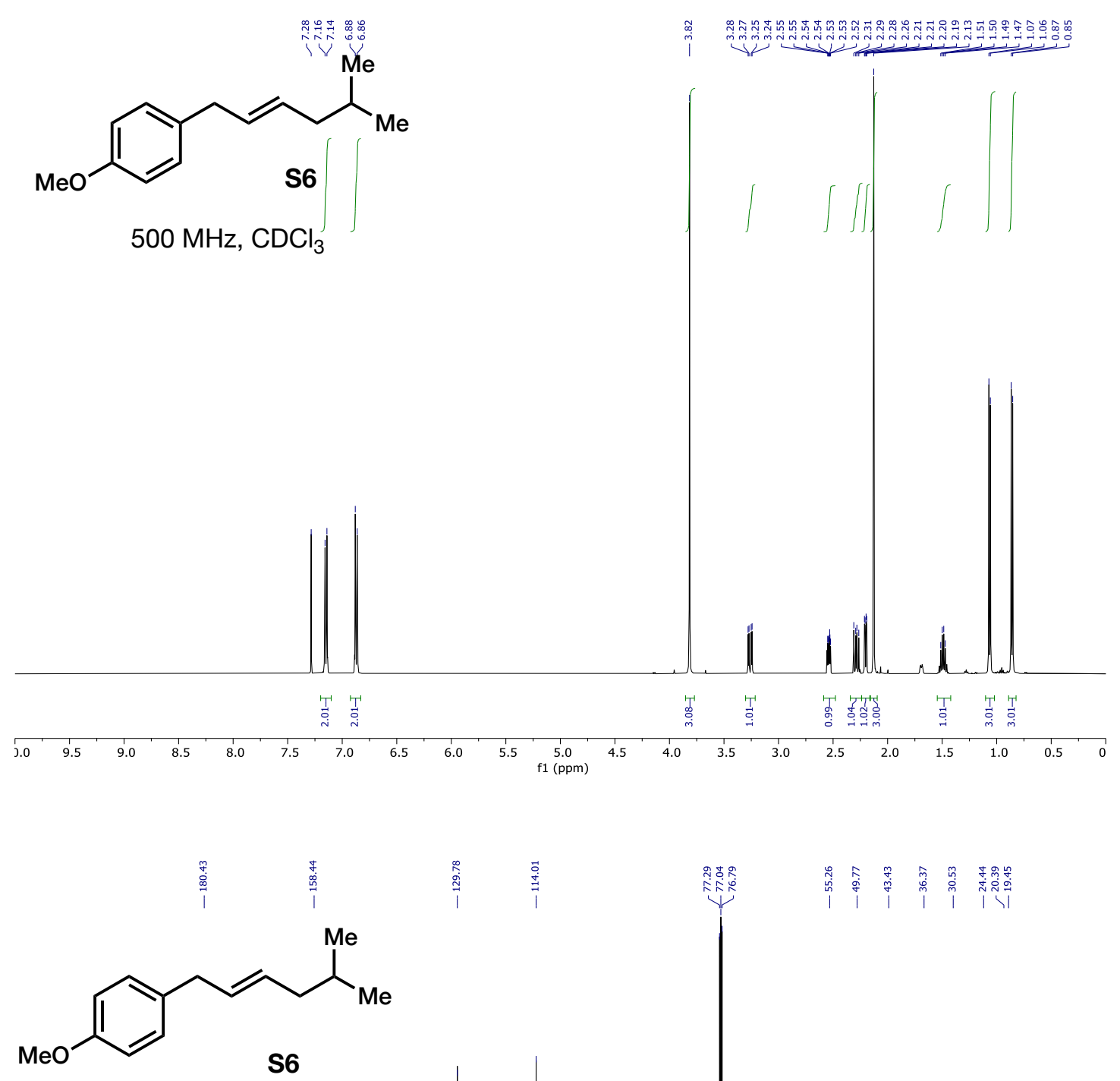

$126 \mathrm{MHz}, \mathrm{CDCl}_{3}$

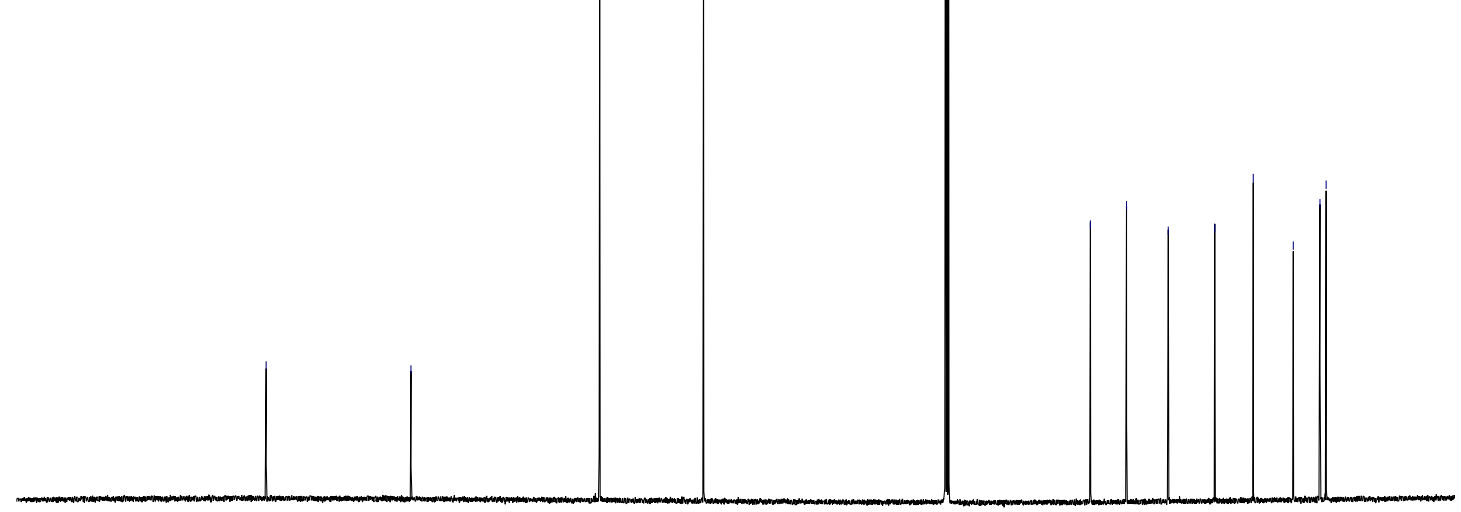

\begin{tabular}{llllllllllllllllllllll}
1 & 1 \\
\hline 210 & 200 & 190 & 180 & 170 & 160 & 150 & 140 & 130 & 120 & $\begin{array}{c}1 \\
\mathrm{f} 1\end{array}(\mathrm{ppm})$ & 100 & 90 & 80 & 70 & 60 & 50 & 40 & 30 & 20 & 10 & 1
\end{tabular} 
<smiles>COc1ccc(CC=CC(C)C)cc1</smiles>

\section{(E)-1-Methoxy-4-(4-methylpent-2-en-1-yl)benzene S7}

General Procedure A: 3-Methylbut-1-yne $(1.5 \mathrm{~mL}, 14.9 \mathrm{mmol})$ and 4-methoxybenzyl bromide $(3.00 \mathrm{~g}, 14.9 \mathrm{mmol})$ were employed. Purification of the residue by column chromatography $(20: 1$, hexanes-EtOAc) afforded the title compound $(2.48 \mathrm{~g}, 87 \%)$ as a colorless oil.

${ }^{1} \mathrm{H}$ NMR $\left(500 \mathrm{MHz}, \mathrm{CDCl}_{3}\right) \delta 7.13(\mathrm{~d}, \mathrm{~J}=8.6 \mathrm{~Hz}, 2 \mathrm{H}), 6.90-6.82(\mathrm{~m}, 2 \mathrm{H}), 5.58-5.46(\mathrm{~m}, 2 \mathrm{H}), 3.82$ (s, 3H), $3.29(\mathrm{~d}, \mathrm{~J}=5.2 \mathrm{~Hz}, 2 \mathrm{H}), 2.37-2.25(\mathrm{~m}, 1 \mathrm{H}), 1.02(\mathrm{~d}, \mathrm{~J}=6.8 \mathrm{~Hz}, 6 \mathrm{H})$;

${ }^{13} \mathrm{C} \mathrm{NMR}\left(126 \mathrm{MHz}_{\mathrm{CDCl}}\right.$ ) $\delta$ 157.8, 138.9, 133.2, 129.3, 126.1, 113.7, 55.3, 38.1, 30.9, 22.6;

IR ( $\mathrm{NaCl}$, thin film) 2870, 2835, 1609, 1511, $1464 \mathrm{~cm}^{-1}$;

HRMS $\left(\mathrm{EI}^{+}\right) \mathrm{m} / \mathrm{z}$ calcd for $\mathrm{C}_{13} \mathrm{H}_{18} \mathrm{O}^{+}(\mathrm{M})^{+}:$190.1352, found 190.1350 . 

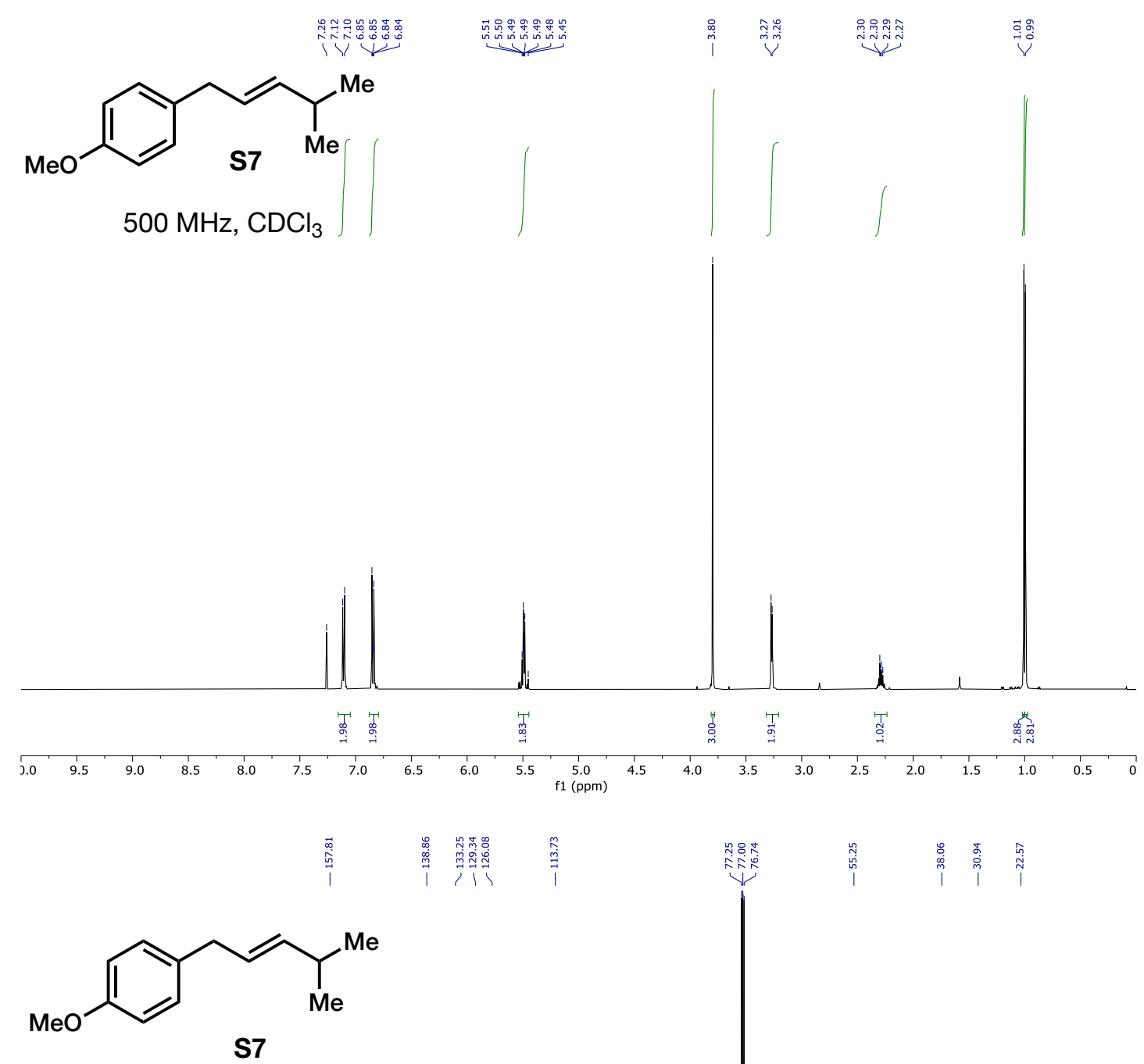

$126 \mathrm{MHz}, \mathrm{CDCl}_{3}$

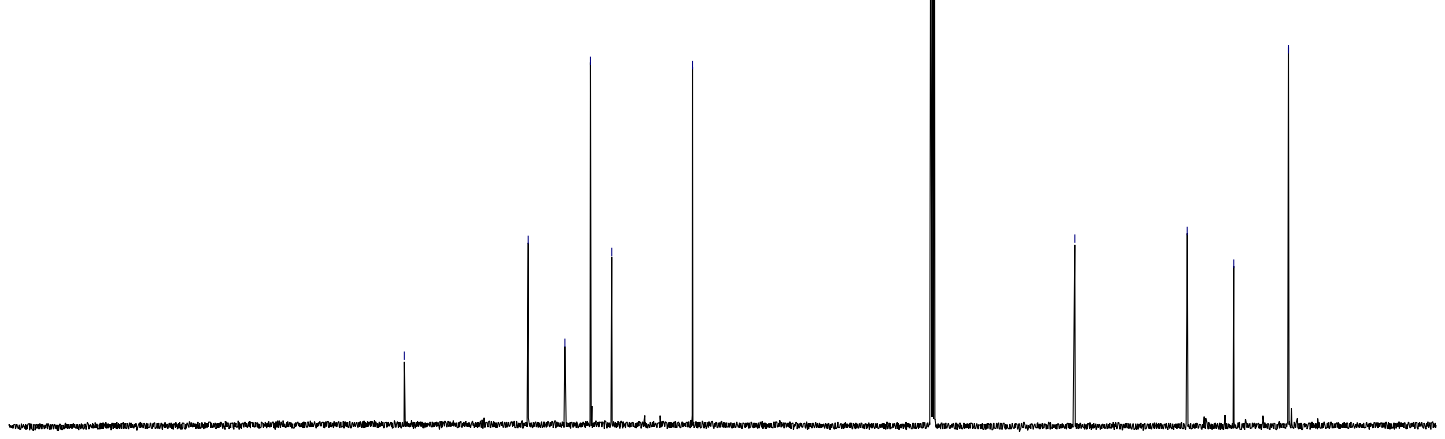


<smiles></smiles>

(E)-4-(4-(4-Methoxyphenyl)but-2-en-1-yl)-1-tosylpiperidine S8

General Procedure A: 4-(Prop-2-yn-1-yl)-1-tosylpiperidine $(2.55 \mathrm{~g}, \quad 9.2 \mathrm{mmol})$ and 4-methoxybenzyl bromide $(1.85 \mathrm{~g}, 9.2 \mathrm{mmol})$ were employed. Purification of the residue by column chromatography (10-25\%, ethyl acetate in hexanes) afforded the title compound (1.53 $\mathrm{g}$, $42 \%)$ as a yellow oil.

${ }^{1} \mathrm{H}$ NMR $\left(500 \mathrm{MHz}, \mathrm{CDCl}_{3}\right) \delta 7.64(\mathrm{~d}, J=8.3 \mathrm{~Hz}, 2 \mathrm{H}), 7.31(\mathrm{~d}, J=8.6 \mathrm{~Hz}, 2 \mathrm{H}), 7.05(\mathrm{~d}, J=8.6$, $\mathrm{Hz}, 2 \mathrm{H}), 6.82(\mathrm{~d}, J=8.6 \mathrm{~Hz}, 2 \mathrm{H}), 5.53(\mathrm{dtt}, J=14.8,6.7,1.3 \mathrm{~Hz}, 1 \mathrm{H}), 5.48(\mathrm{dtt}, J=15.5,7.1,1.4$ $\mathrm{Hz}, 1 \mathrm{H}), 3.78(\mathrm{~s}, 3 \mathrm{H}), 3.75(\mathrm{dd}, J=11.6,2.7 \mathrm{~Hz}, 2 \mathrm{H}), 3.24(\mathrm{~d}, J=6.7 \mathrm{~Hz}, 2 \mathrm{H}), 2.43(\mathrm{~s}, 3 \mathrm{H}), 2.21$ (td, $J=11.6,2.5 \mathrm{~Hz}, 2 \mathrm{H}$ ), 1.93 (app. t, $J=6.8 \mathrm{~Hz}, 2 \mathrm{H}), 1.72-1.67(\mathrm{~m}, 2 \mathrm{H}), 1.33-1.19(\mathrm{~m}, 3 \mathrm{H})$;

${ }^{13} \mathrm{C}$ NMR $\left(126 \mathrm{MHz}, \mathrm{CDCl}_{3}\right) \delta 157.9,143.3,133.2,132.7,131.5,129.5,129.3,128.5,127.7$, 113.8, 55.2, 46.4, 39.0, 38.1, 35.5, 31.2, 21.5;

IR ( $\mathrm{NaCl}$, thin film) 2913, 2836, 1609, 1511, $1245 \mathrm{~cm}^{-1}$;

HRMS $\left(\mathrm{Cl}^{+}\right) \mathrm{m} / \mathrm{z}$ calcd for $\mathrm{C}_{23} \mathrm{H}_{29} \mathrm{O}_{3} \mathrm{NSNH}_{4}\left(\mathrm{M}+\mathrm{NH}_{4}\right)^{+}:$417.2206, found 417.2198. 


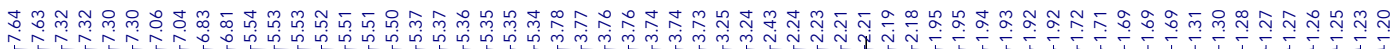

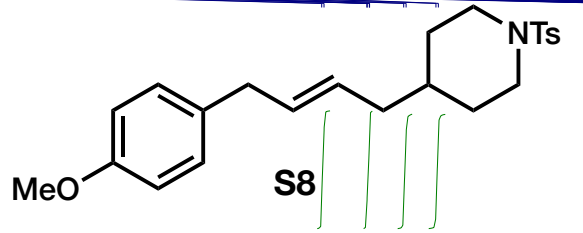

$500 \mathrm{MHz}, \mathrm{CDCl}_{3}$
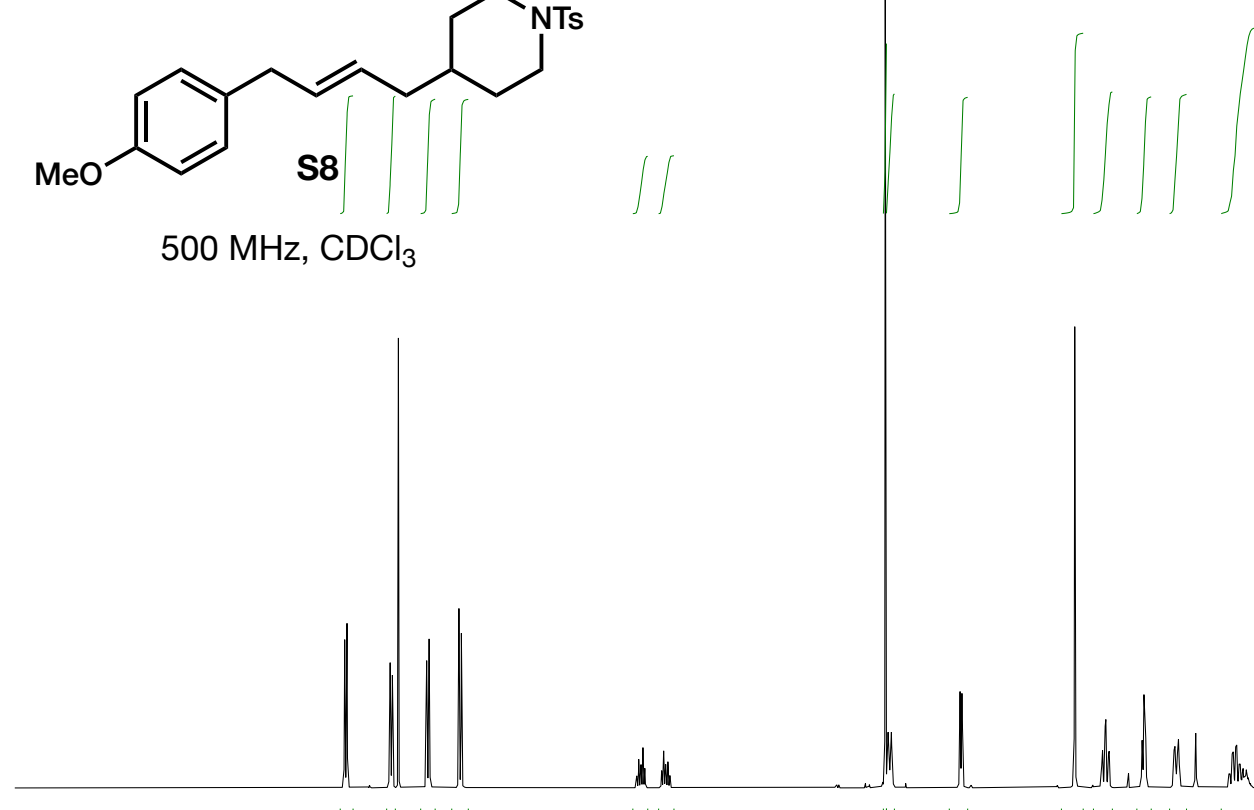

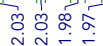

u

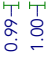

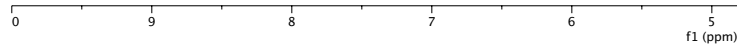

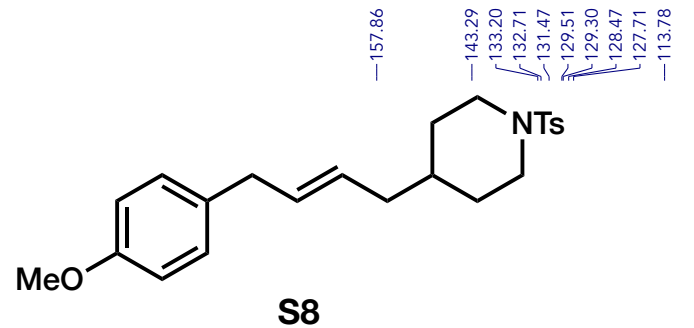

岂

$126 \mathrm{MHz}, \mathrm{CDCl}_{3}$

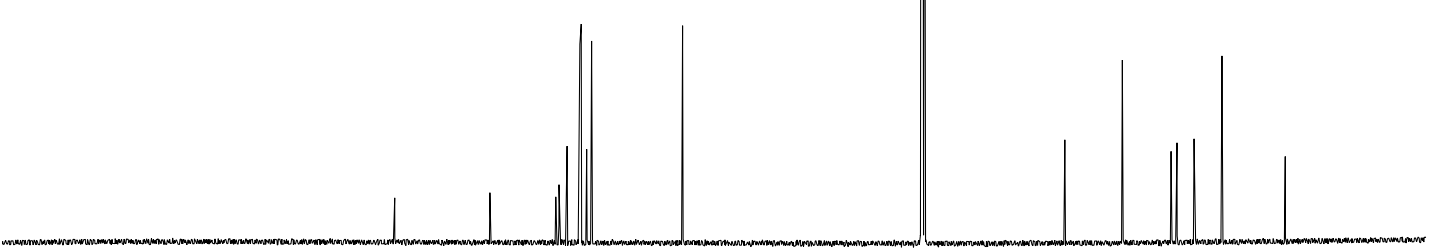

200

180

160

140

$120 \quad \mathrm{f} 1(\mathrm{ppm}) \quad 100$

80

60 


\section{Aziridine synthesis}<smiles>CCCC1C(Cc2ccc(OC)cc2)N1C(C)=O</smiles>

1-((2R* $\left.\left.3 R^{*}\right)-2-(4-M e t h o x y b e n z y l)-3-p r o p y l a z i r i d i n-1-y l\right) e t h a n-1-o n e$ 5a-Ac

General Procedure C: (E)-1-(Hex-2-en-1-yl)-4-methoxybenzene (835 mg, $4.39 \mathrm{mmol}$ ) was employed. Purification of the residue by column chromatography $(0-20 \%$, ethyl acetate in hexanes) afforded the title compound (674 $\mathrm{mg}, 62 \%)$ as an orange oil.

${ }^{1} \mathrm{H}$ NMR $\left(500 \mathrm{MHz}, \mathrm{CDCl}_{3}\right) \delta 7.14(\mathrm{~d}, J=8.6 \mathrm{~Hz}, 2 \mathrm{H}), 6.85(\mathrm{~d}, J=8.6 \mathrm{~Hz}, 2 \mathrm{H}), 3.80(\mathrm{~s}, 3 \mathrm{H}), 3.06$ (dd, $J=12.8,3.43 \mathrm{~Hz}, 1 \mathrm{H}$ ), 2.50-2.44 (m, 2H), 2.38 (ddd, $J=7.7,4.9,2.5 \mathrm{~Hz}, 1 \mathrm{H}), 2.05(\mathrm{~s}, 3 \mathrm{H}$ ), $1.82-1.75(\mathrm{~m}, 1 \mathrm{H}), 1.49-1.30(\mathrm{~m}, 2 \mathrm{H}), 1.18-1.10(\mathrm{~m}, 1 \mathrm{H}), 0.88(\mathrm{t}, J=7.4 \mathrm{~Hz}, 3 \mathrm{H})$;

${ }^{13} \mathrm{C} \mathrm{NMR}\left(126 \mathrm{MHz} \mathrm{CDCl}_{3}\right) \delta 180.8,158.4,129.9,129.7,113.9,55.2,44.0,43.6,36.8,33.3,24.4$, 20.5, 13.7;

IR ( $\mathrm{NaCl}$, thin film) 2959, 2931, 1684, 1513, $1248 \mathrm{~cm}^{-1}$;

HRMS (ESI-TOF) $\mathrm{m} / \mathrm{z}$ calcd for $\mathrm{C}_{15} \mathrm{H}_{21} \mathrm{O}_{2} \mathrm{NNa}(\mathrm{M}+\mathrm{Na})^{+}: 270.1465$, found 270.1464 . 


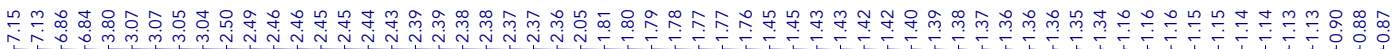
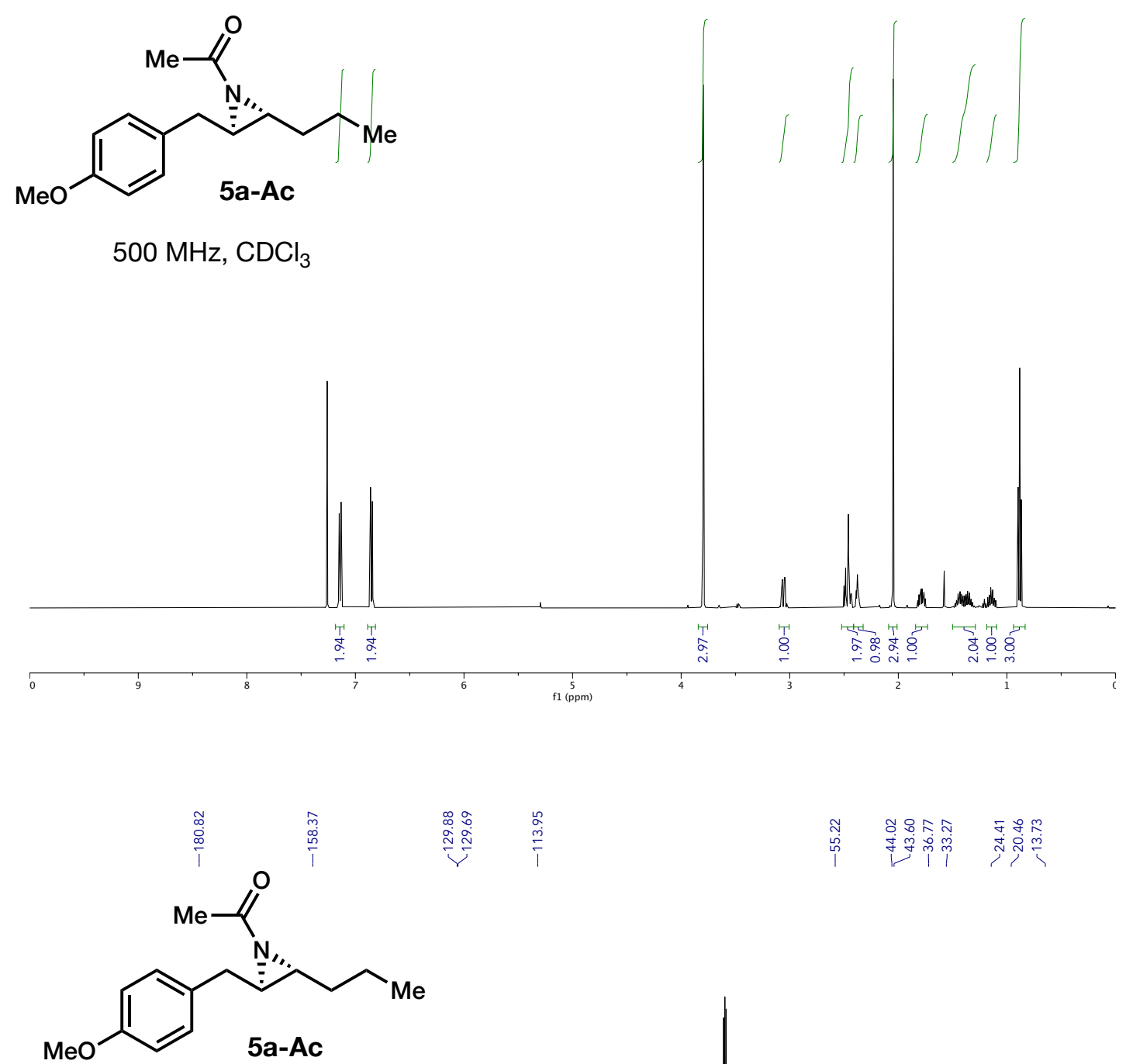

$126 \mathrm{MHz}, \mathrm{CDCl}_{3}$

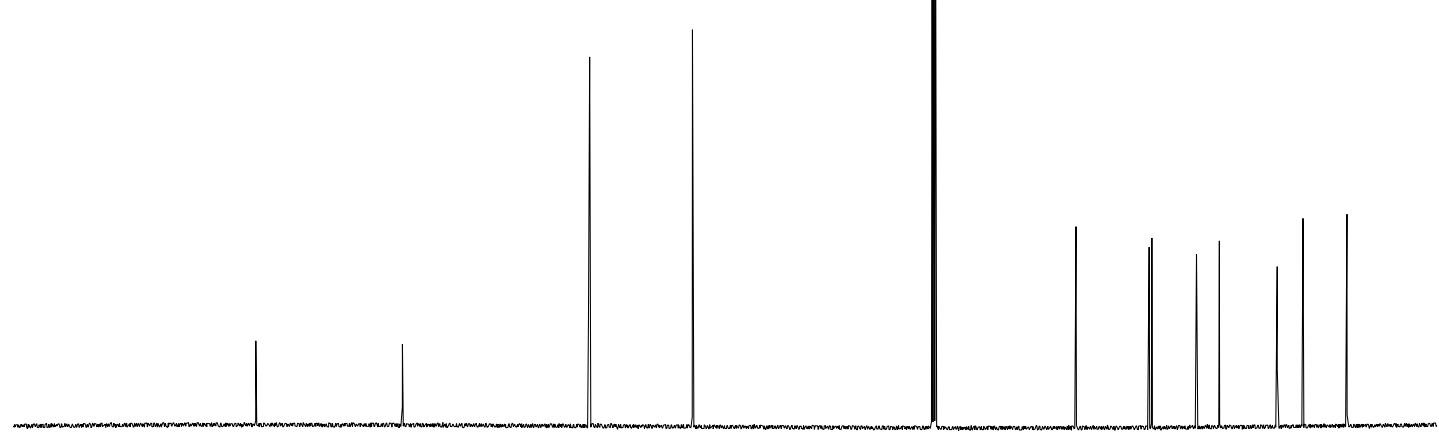




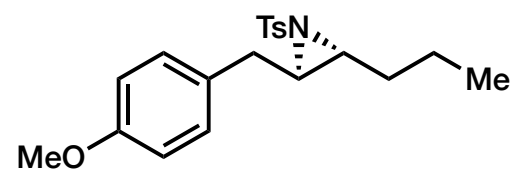

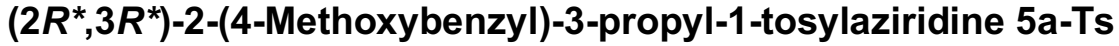

General Procedure B: (E)-1-(Hex-2-en-1-yl)-4-methoxybenzene (800 mg, $4.20 \mathrm{mmol}$ ) was employed. Purification of the residue by column chromatography $(0-20 \%$, ethyl acetate in hexanes) afforded the title compound $(1.33 \mathrm{~g}, 88 \%)$ as a pale yellow oil. This material contains approximately $6 \%$ of the corresponding cis-aziridine.

${ }^{1} \mathrm{H}$ NMR $\left(400 \mathrm{MHz}, \mathrm{C}_{6} \mathrm{D}_{6}\right) \delta 7.92(\mathrm{~d}, J=8.3 \mathrm{~Hz}, 2 \mathrm{H}), 6.82(\mathrm{~d}, J=8.4 \mathrm{~Hz}, 2 \mathrm{H}), 6.74(\mathrm{~d}, J=8.0 \mathrm{~Hz}$, $2 \mathrm{H}), 6.45 \mathrm{f}(\mathrm{d}, J=8.6 \mathrm{~Hz}, 2 \mathrm{H}), 3.30(\mathrm{~s}, 3 \mathrm{H}), 2.96-2.9 \mathrm{o}(\mathrm{m}, 2 \mathrm{H}), 2.82$ (app. td, $J=6.5,4.3 \mathrm{~Hz}, 1 \mathrm{H})$, 2.65 (app. td, $J=6.6,4.3 \mathrm{~Hz}, 1 \mathrm{H}), 1.88(\mathrm{~s}, 3 \mathrm{H}), 1.81-1.71(\mathrm{~m}, 1 \mathrm{H}), 1.62-1.53(\mathrm{~m}, 1 \mathrm{H}), 1.28-1.06$ $(\mathrm{m}, 2 \mathrm{H}), 0.67(\mathrm{t}, J=7.4 \mathrm{~Hz}, 3 \mathrm{H})$;

${ }^{13} \mathrm{C}$ NMR $\left(101 \mathrm{MHz}, \mathrm{C}_{6} \mathrm{D}_{6}\right) \delta 159.0,143.3,139.3,130.2,130.0,129.5,127.9,114.3,54.8,50.2$, 49.5, 35.8, 32.1, 21.2, 21.1, 13.8;

IR ( $\mathrm{NaCl}$, thin film) 2872, 2836, 1584, $1248,1178 \mathrm{~cm}^{-1}$;

HRMS $\left(\mathrm{Cl}^{+}\right) \mathrm{m} / \mathrm{z}$ calcd for $\mathrm{C}_{20} \mathrm{H}_{25} \mathrm{O}_{3} \mathrm{NSNH}_{4}\left(\mathrm{M}+\mathrm{NH}_{4}\right)^{+}: 377.1893$, found 377.1883 . 



$101 \mathrm{MHz}, \mathrm{C}_{6} \mathrm{D}_{6}$

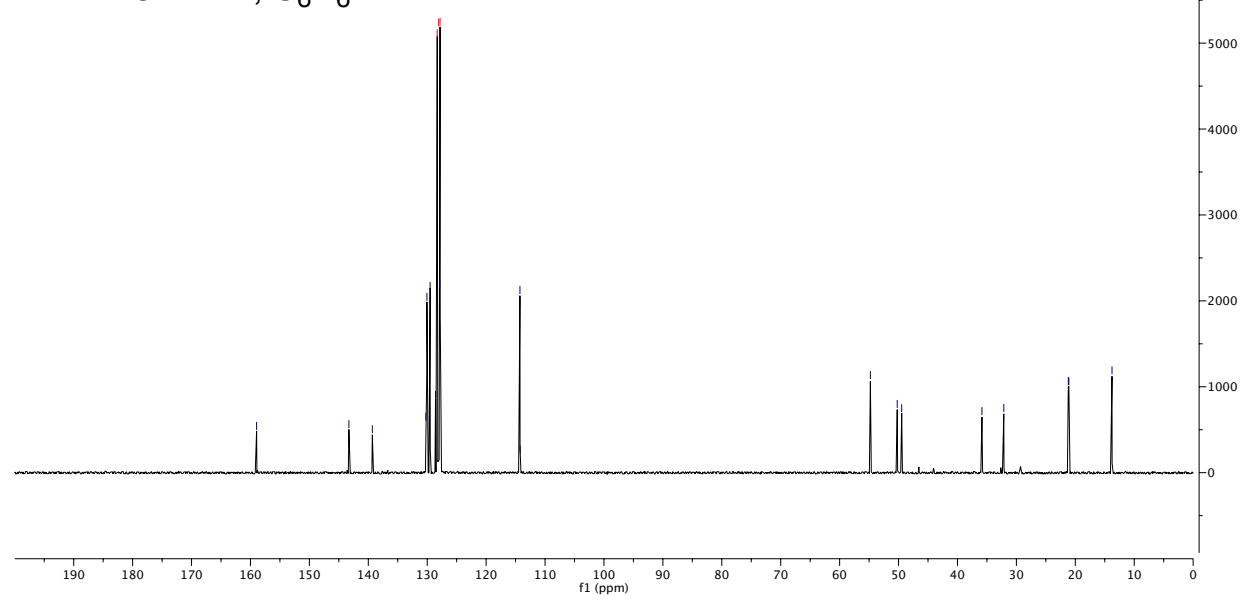




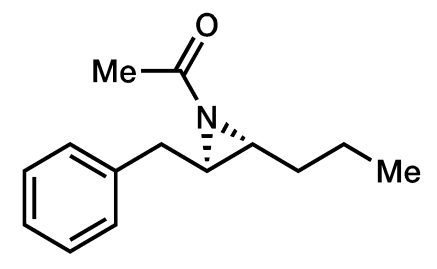

1-((2R*, $\left.\left.3 R^{*}\right)-2-B e n z y l-3-p r o p y l a z i r i d i n-1-y l\right) e t h a n-1-o n e ~ 5 b-A c$

General Procedure C: (E)-Hex-2-en-1-ylbenzene $(2.10 \mathrm{~g}, 13.08 \mathrm{mmol})$ was employed. Purification of the residue by column chromatography $(0-25 \%$, ethyl acetate in hexanes) afforded the title compound $(2.02 \mathrm{~g}, 71 \%)$ as a dark orange oil.

${ }^{1} \mathrm{H}$ NMR $\left(500 \mathrm{MHz}, \mathrm{CDCl}_{3}\right) \delta$ 7.34-7.30 (m, 2H), 7.24-7.21 (m, 3H), $3.13(\mathrm{dd}, \mathrm{J}=13.1,3.9 \mathrm{~Hz}$, $1 \mathrm{H}), 2.55-2.46(\mathrm{~m}, 2 \mathrm{H}), 2.40$ (ddd, $J=7.6,4.9,2.6 \mathrm{~Hz}, 1 \mathrm{H}), 2.05(\mathrm{~s}, 3 \mathrm{H}), 1.83-1.75(\mathrm{~m}, 1 \mathrm{H}), 1.48-$ $1.30(\mathrm{~m}, 2 \mathrm{H}), 1.19-1.10(\mathrm{~m}, 1 \mathrm{H}), 0.87(\mathrm{t}, J=7.4 \mathrm{~Hz}, 3 \mathrm{H})$;

${ }^{13} \mathrm{C}$ NMR $\left(126 \mathrm{MHz} \mathrm{CDCl}_{3}\right) \delta 180.8,137.9,128.7,128.6,126.7,43.8,43.7,37.7,33.3,24.4,20.4$, 13.7

IR ( $\mathrm{NaCl}$, thin film) 2961, 2931, 1686, 1455, 1368, $1185 \mathrm{~cm}^{-1}$;

HRMS (ESI-TOF) $\mathrm{m} / \mathrm{z}$ calcd for $\mathrm{C}_{14} \mathrm{H}_{19} \mathrm{ONNa}(\mathrm{M}+\mathrm{Na})^{+}:$240.1364, found 240.1361. 


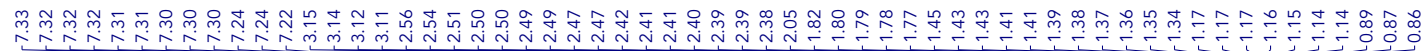
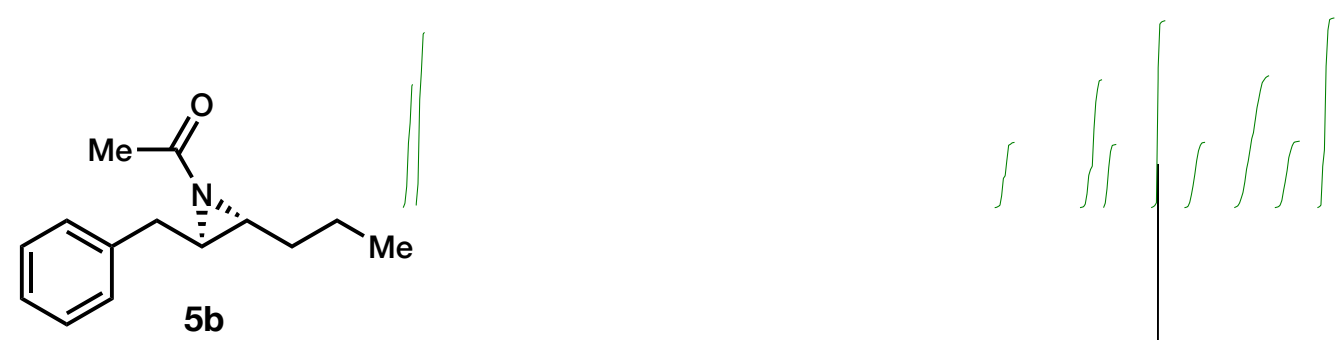

$500 \mathrm{MHz}, \mathrm{CDCl}_{3}$
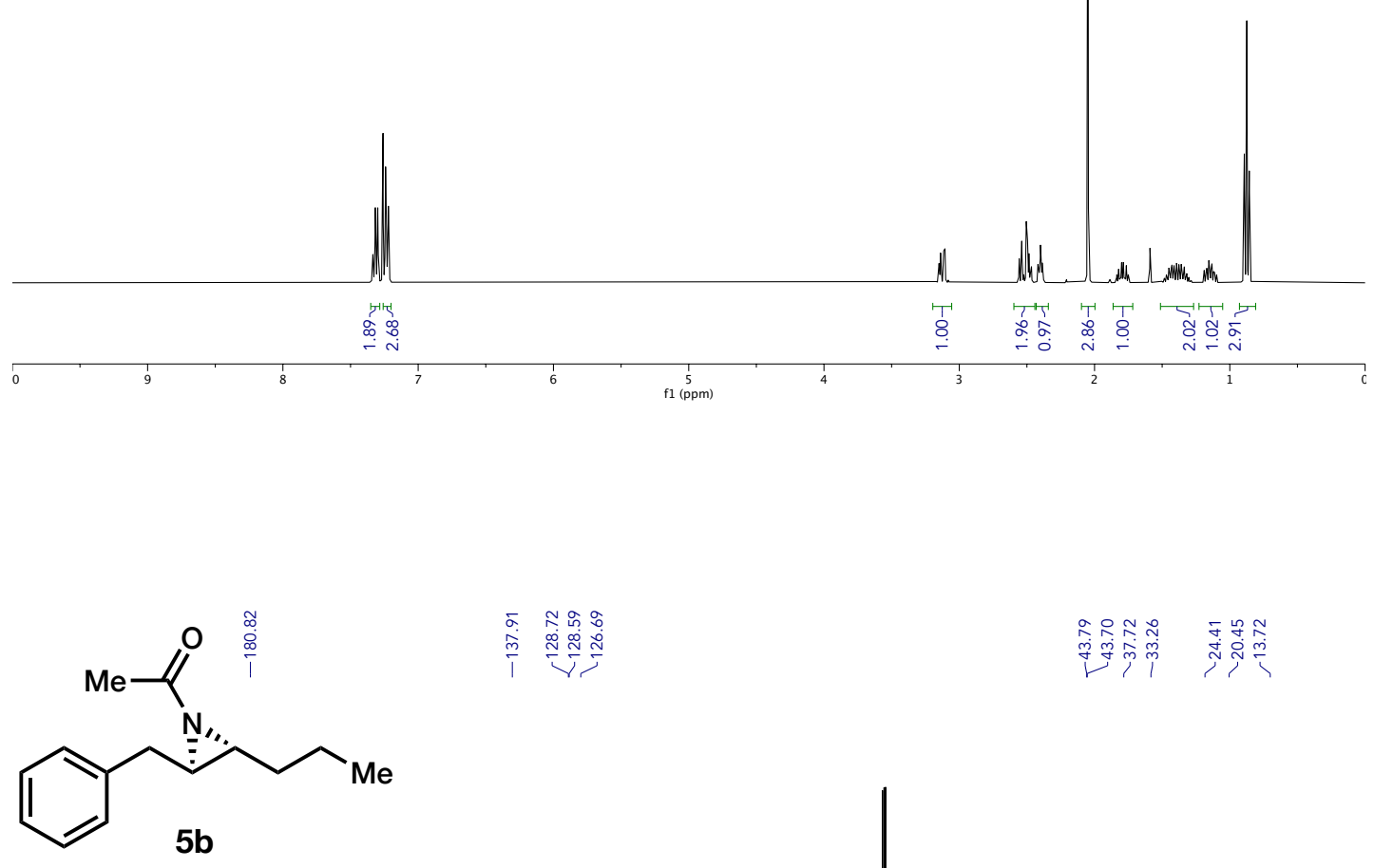

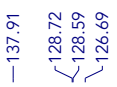

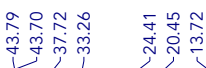

$126 \mathrm{MHz}, \mathrm{CDCl}_{3}$
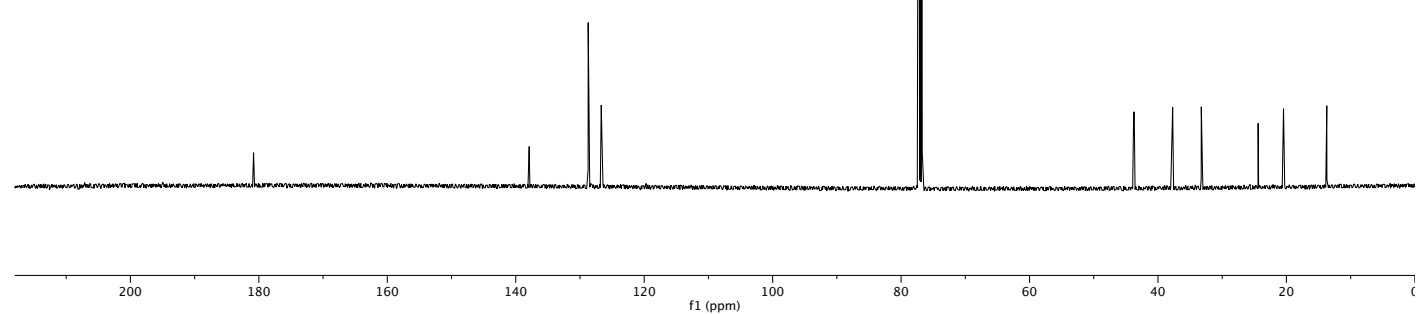
<smiles>CCCC1C(Cc2ccc(C)cc2)N1C(C)=O</smiles>

1-((2R*,3R*)-2-(4-Methylbenzyl)-3-propylaziridin-1-yl)ethan-1-one 5c

General Procedure C: (E)-1-(Hex-2-en-1-yl)-4-methylbenzene (1.49 g, $8.55 \mathrm{mmol})$ was employed. Purification of the residue by column chromatography $(5-40 \%$, ethyl acetate in hexanes) afforded the title compound (1.28 g, 65\%) as an orange oil.

${ }^{1} \mathrm{H}$ NMR $\left(500 \mathrm{MHz}, \mathrm{CDCl}_{3}\right) \delta 7.11(\mathrm{~s}, 4 \mathrm{H}), 3.11-3.06(\mathrm{~m}, 1 \mathrm{H}), 2.50-2.45(\mathrm{~m}, 2 \mathrm{H}), 2.39$ (ddd, $J=$ 7.5, 5.0, 2.4 Hz, 1H), $2.33(\mathrm{~s}, 3 \mathrm{H}), 2.05(\mathrm{~s}, 3 \mathrm{H}), 1.81-1.74(\mathrm{~m}, 1 \mathrm{H}), 1.48-1.32(\mathrm{~m}, 2 \mathrm{H}), 1.18-1.11$ $(\mathrm{m}, 1 \mathrm{H}), 0.88(\mathrm{t}, J=7.4 \mathrm{~Hz}, 3 \mathrm{H})$;

${ }^{13} \mathrm{C}$ NMR $\left(126 \mathrm{MHz} \mathrm{CDCl}_{3}\right) \delta 180.8,136.2,134.7,129.2,128.6,43.9,43.7,37.2,33.3,24.4,21.0$, 20.5, 13.7;

IR ( $\mathrm{NaCl}$, thin film) 2960, 2929, 1687, 1444, 1366, $1316 \mathrm{~cm}^{-1}$;

HRMS (ESI-TOF) $\mathrm{m} / \mathrm{z}$ calcd for $\mathrm{C}_{15} \mathrm{H}_{21} \mathrm{ONNa}(\mathrm{M}+\mathrm{Na})^{+}: 254.1521$, found 254.1526. 


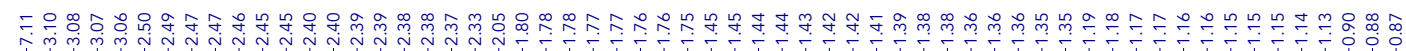
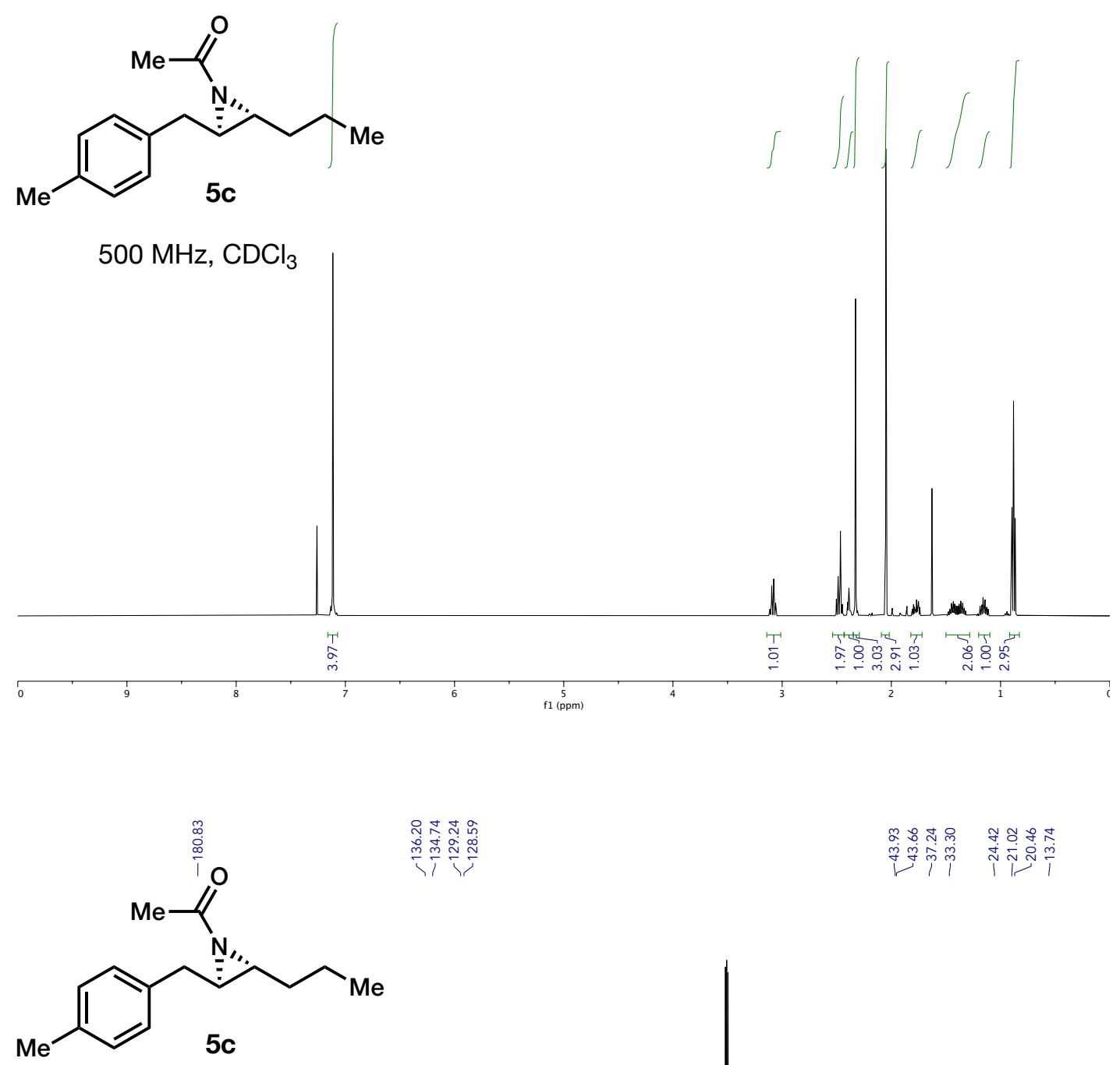

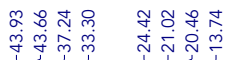

产

मां।

$126 \mathrm{MHz}, \mathrm{CDCl}_{3}$

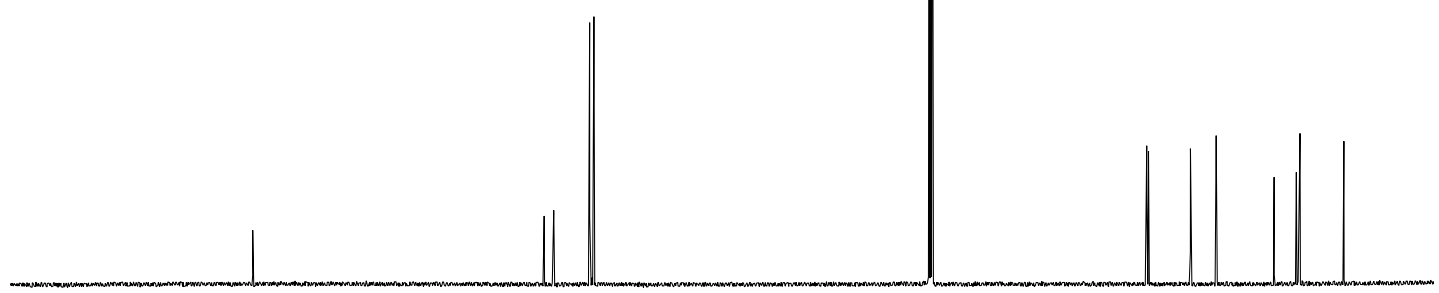

$200 \quad 180$

160 140

$120 \quad \mathrm{f} 1(\mathrm{ppm}) \quad 100$

80

60 
<smiles>CCCC1C(Cc2ccc(C)c(C)c2)N1C(C)=O</smiles>

\section{1-((2R*,3R*)-2-(3,4-Dimethylbenzyl)-3-propylaziridin-1-yl)ethan-1-one 5d}

General Procedure C: (E)-4-(Hex-2-en-1-yl)-1,2-dimethylbenzene (1.90 g, $10.09 \mathrm{mmol})$ was employed. Purification of the residue by column chromatography $(0-35 \%$, ethyl acetate in hexanes) afforded the title compound (449 $\mathrm{mg}, 18 \%$ ) as an orange oil.

${ }^{1} \mathrm{H}$ NMR $\left(500 \mathrm{MHz}, \mathrm{CDCl}_{3}\right) \delta 7.07(\mathrm{~d}, J=7.6 \mathrm{~Hz}, 1 \mathrm{H}), 6.99(\mathrm{~d}, J=1.9 \mathrm{~Hz}, 1 \mathrm{H}), 6.95(\mathrm{dd}, J=7.7$, $1.9 \mathrm{~Hz}, 1 \mathrm{H}), 3.10-3.03(\mathrm{~m}, 1 \mathrm{H}), 2.48-2.38(\mathrm{~m}, 3 \mathrm{H}), 2.25(\mathrm{~s}, 3 \mathrm{H}), 2.24(\mathrm{~s}, 3 \mathrm{H}), 2.06(\mathrm{~s}, 3 \mathrm{H}), 1.80-$ $1.74(\mathrm{~m}, 1 \mathrm{H}), 1.49-1.33(\mathrm{~m}, 2 \mathrm{H}), 1.20-1.13(\mathrm{~m}, 1 \mathrm{H}), 0.89(\mathrm{t}, J=7.4 \mathrm{~Hz}, 3 \mathrm{H})$;

${ }^{13} \mathrm{C}$ NMR $\left(126 \mathrm{MHz}, \mathrm{CDCl}_{3}\right) \delta 180.8,136.7,135.2,134.8,130.0,129.8,126.0,44.0,43.7,37.2$, $33.3,24.4,20.5,19.7,19.3,13.7$;

IR ( $\mathrm{NaCl}$, thin film) 2959, 2929, 1687, 1446, 1366, $1318 \mathrm{~cm}^{-1}$;

HRMS (ESI-TOF) $\mathrm{m} / \mathrm{z}$ calcd for $\mathrm{C}_{16} \mathrm{H}_{23} \mathrm{ONNa}(\mathrm{M}+\mathrm{Na})^{+}:$268.1677, found 268.1677. 

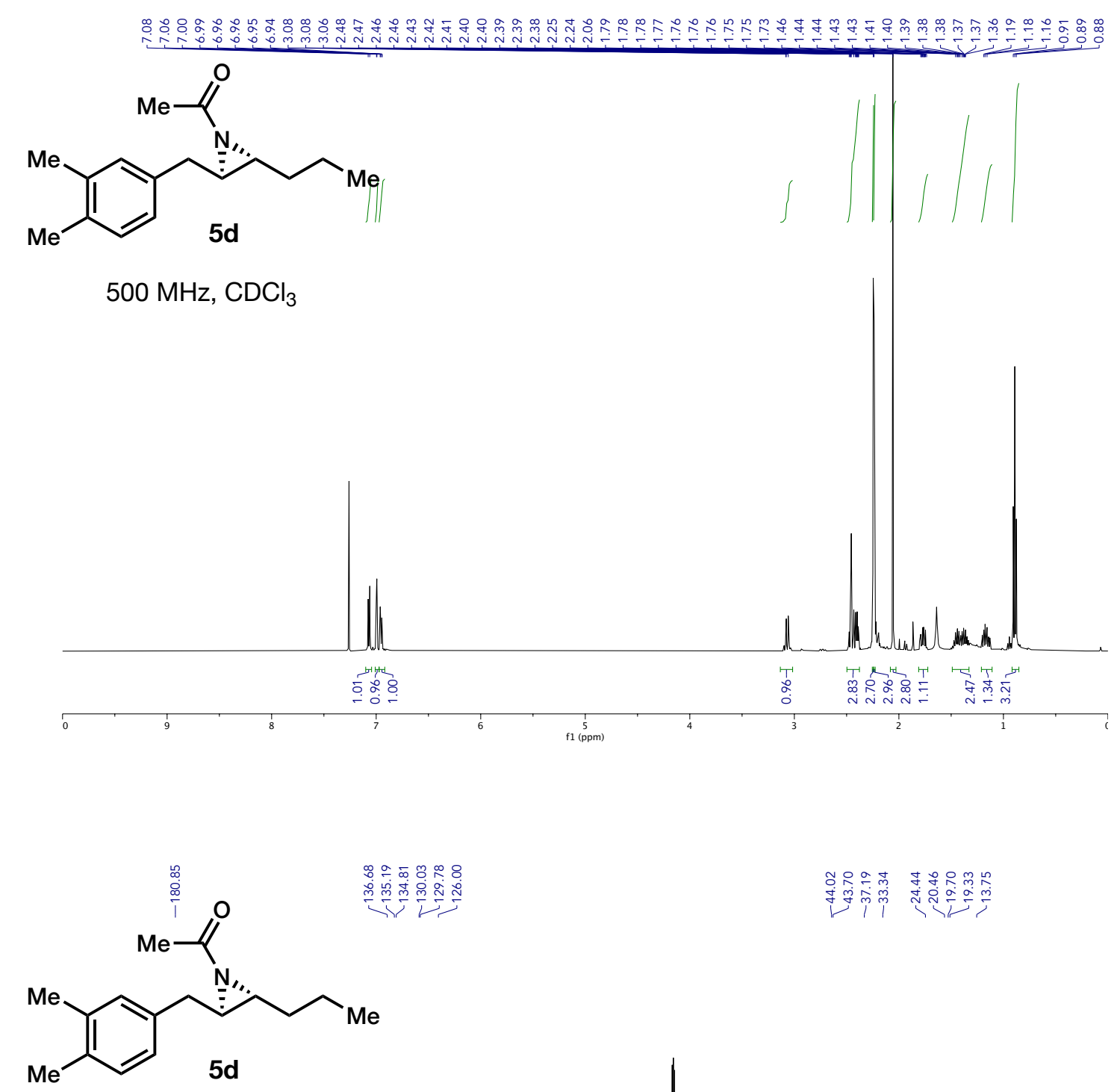

$126 \mathrm{MHz}, \mathrm{CDCl}_{3}$

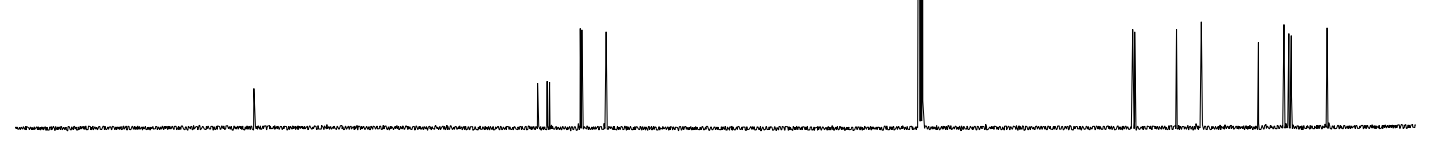

200 180

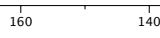

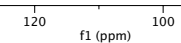

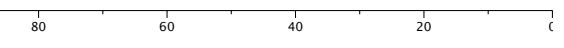


<smiles>CCCC1[C@H](Cc2ccc(OC)c(OC)c2)N1C(C)=O</smiles>

\section{1-((2R*, $\left.\left.3 R^{*}\right)-2-(3,4-D i m e t h o x y b e n z y l)-3-p r o p y l a z i r i d i n-1-y l\right) e t h a n-1-o n e ~ 5 e$}

General Procedure C: (E)-4-(Hex-2-en-1-yl)-1,2-dimethoxybenzene (597 mg, $2.71 \mathrm{mmol})$ was employed. Purification of the residue by column chromatography $(0-35 \%$, ethyl acetate in hexanes) afforded the title compound (340 $\mathrm{mg}, 45 \%)$ as a pale yellow oil.

${ }^{1} \mathrm{H}$ NMR $\left(500 \mathrm{MHz}, \mathrm{CDCl}_{3}\right) \delta 6.81(\mathrm{~d}, J=8.1 \mathrm{~Hz}, 1 \mathrm{H}), 6.78-6.75(\mathrm{~m}, 2 \mathrm{H}), 3.88(\mathrm{~s}, 3 \mathrm{H}), 3.87(\mathrm{~s}$, $3 \mathrm{H}$ ), 3.03 (dd, $J=14.0,4.8 \mathrm{~Hz}, 1 \mathrm{H}), 2.54-2.45(\mathrm{~m}, 2 \mathrm{H}), 2.39$ (ddd, $J=7.7,4.9,2.7 \mathrm{~Hz}, 1 \mathrm{H}), 2.06$ $(\mathrm{s}, 3 \mathrm{H}), 1.84-1.78(\mathrm{~m}, 1 \mathrm{H}), 1.50-1.33(\mathrm{~m}, 2 \mathrm{H}), 1.17-1.09(\mathrm{~m}, 1 \mathrm{H}), 0.89(\mathrm{t}, J=7.4 \mathrm{~Hz}, 3 \mathrm{H})$;

${ }^{13} \mathrm{C}$ NMR $\left(126 \mathrm{MHz}, \mathrm{CDCl}_{3}\right) \delta 180.9,148.9,147.8,130.4,120.7,112.0,111.3,55.9,55.8,43.9$, 43.6, 37.2, 33.3, 24.4, 20.5, 13.8;

IR (NaCl, thin film) 2959, 2993, 1682, 1464, 1367, $1320 \mathrm{~cm}^{-1}$;

HRMS (ESI-TOF) $m / z$ calcd for $\mathrm{C}_{16} \mathrm{H}_{23} \mathrm{O}_{3} \mathrm{NNa}(\mathrm{M}+\mathrm{Na})^{+}: 300.1576$, found 300.1570 . 

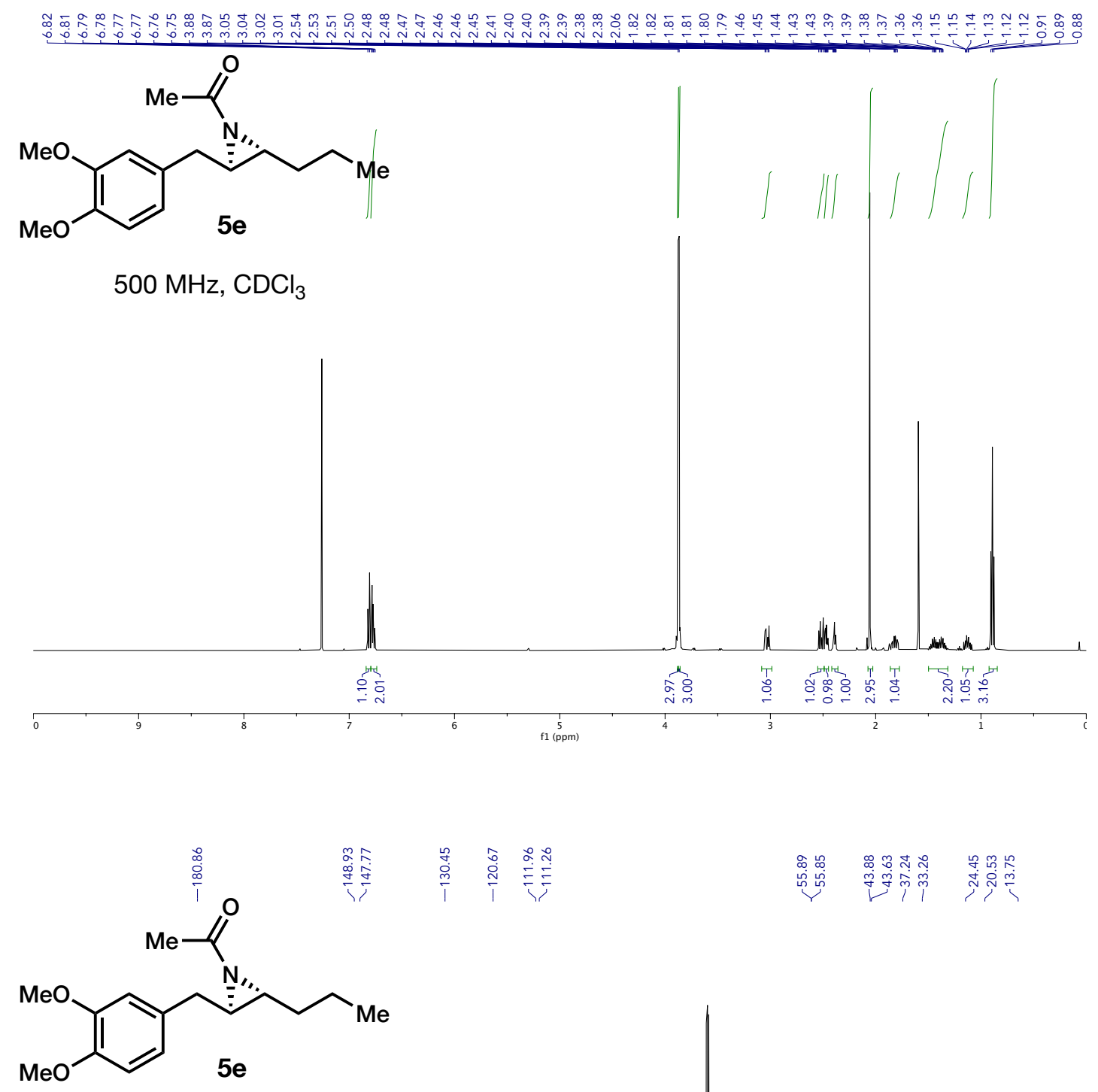

$126 \mathrm{MHz}, \mathrm{CDCl}_{3}$

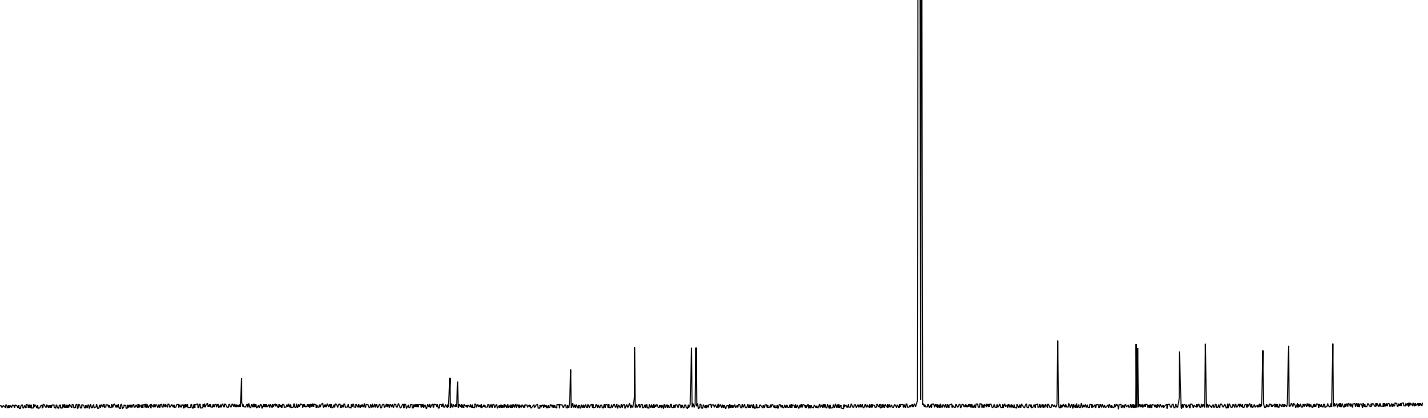


<smiles>CCCC1NC(Cc2ccc(OC)c(Br)c2)[C@@H]1C(C)=O</smiles>

1-((2R*,3R*)-2-(3-Bromo-4-methoxybenzyl)-3-propylaziridin-1-yl)ethan-1-one 5f

General Procedure C: (E)-2-Bromo-4-(hex-2-en-1-yl)-1-methoxybenzene (1.00 g, $3.71 \mathrm{mmol})$ was employed. Purification of the residue by column chromatography $(1: 1$, hexane:EtOAc) afforded the title compound $(0.97 \mathrm{~g}, 80 \%)$ as a red oil.

${ }^{1} \mathrm{H}$ NMR $\left(400 \mathrm{MHz}, \mathrm{CDCl}_{3}\right) \delta 7.42(\mathrm{~d}, J=2.2 \mathrm{~Hz}, 1 \mathrm{H}), 7.14(\mathrm{dd}, J=8.4,2.2 \mathrm{~Hz}, 1 \mathrm{H}), 6.85(\mathrm{~d}, J=$ $8.4 \mathrm{~Hz}, 1 \mathrm{H}), 3.88(\mathrm{~s}, 3 \mathrm{H}), 3.01(\mathrm{dd}, J=13.9,4.6 \mathrm{~Hz}, 1 \mathrm{H}), 2.54-2.33(\mathrm{~m}, 3 \mathrm{H}), 2.05(\mathrm{~s}, 3 \mathrm{H}), 1.90-$ $1.75(\mathrm{~m}, 1 \mathrm{H}), 1.54-1.28(\mathrm{~m}, 2 \mathrm{H}), 1.19-1.05(\mathrm{~m}, 1 \mathrm{H}), 0.90(\mathrm{t}, J=7.4 \mathrm{~Hz}, 3 \mathrm{H})$;

${ }^{13} \mathrm{C}$ NMR $\left(100 \mathrm{MHz}, \mathrm{CDCl}_{3}\right) \delta 180.8,154.7,133.5,131.5,128.7,112.0,111.6,56.2,43.7,43.5$, 36.4, 33.2, 24.4, 20.5, 13.7;

IR $\left(\mathrm{NaCl}\right.$, thin film) 2959, 2930, 1683, 1497, 1441, $1256 \mathrm{~cm}^{-1}$;

HRMS (ESI-TOF) $\mathrm{m} / \mathrm{z}$ calcd for $\mathrm{C}_{15} \mathrm{H}_{20} \mathrm{BrNO}_{2} \mathrm{H}(\mathrm{M}+\mathrm{H})^{+}: 326.0750$, found 326.0778. 


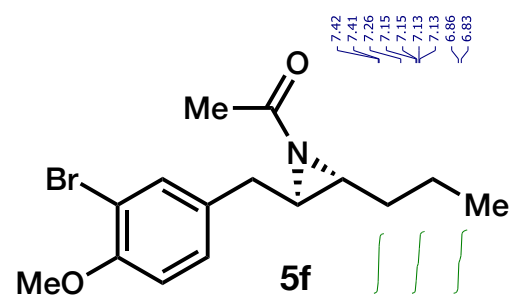

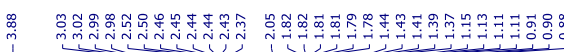

$400 \mathrm{MHz}, \mathrm{CDCl}_{3}$
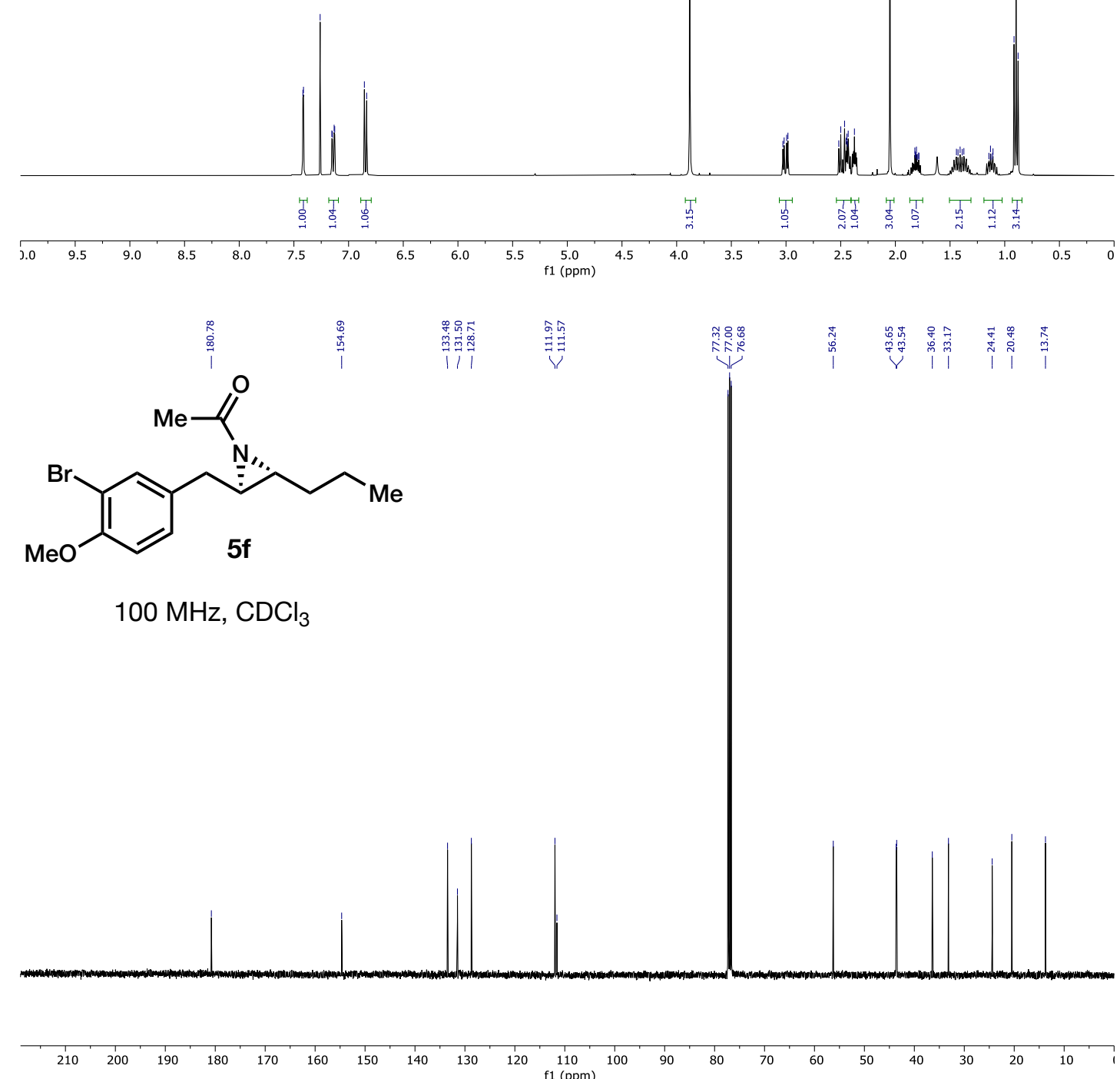
<smiles>CCCC1[C@H](Cc2ccc(N3CCOCC3)cc2)N1C(C)=O</smiles>

\section{1-((2R*,3R*)-2-(4-Morpholinobenzyl)-3-propylaziridin-1-yl)ethan-1-one $5 \mathrm{~g}$}

General Procedure C: (E)-4-(4-(Hex-2-en-1-yl)phenyl)morpholine (1.51 g, $6.15 \mathrm{mmol})$ was employed. Purification of the residue by column chromatography $(1: 1$, hexane:EtOAc with $1 \%$ $\left.\mathrm{Et}_{3} \mathrm{~N}\right)$ afforded the title compound $(0.48 \mathrm{~g}, 26 \%)$ as a black oil.

${ }^{1} \mathrm{H}$ NMR $\left(500 \mathrm{MHz}, \mathrm{CDCl}_{3}\right) \delta 7.13(\mathrm{~d}, J=8.6 \mathrm{~Hz}, 2 \mathrm{H}), 6.86(\mathrm{~d}, J=8.6 \mathrm{~Hz}, 2 \mathrm{H}), 3.90-3.81(\mathrm{~m}, 4 \mathrm{H})$, $3.13(\mathrm{td}, J=4.6,1.9 \mathrm{~Hz}, 4 \mathrm{H}), 3.08-2.99(\mathrm{~m}, 1 \mathrm{H}), 2.51-2.43(\mathrm{~m}, 2 \mathrm{H}), 2.41-2.34(\mathrm{~m}, 1 \mathrm{H}), 2.04(\mathrm{~s}$, $3 \mathrm{H}), 1.81-1.74(\mathrm{~m}, 1 \mathrm{H}), 1.51-1.29(\mathrm{~m}, 2 \mathrm{H}), 1.20-1.09(\mathrm{~m}, 1 \mathrm{H}), 0.88(\mathrm{t}, J=7.4 \mathrm{~Hz}, 3 \mathrm{H})$;

${ }^{13} \mathrm{C}$ NMR $\left(126 \mathrm{MHz} \mathrm{CDCl}_{3}\right) \delta 180.8,150.1,129.5,129.3,115.9,66.9,49.5,44.0,43.6,36.7,33.3$, 24.5, 20.5, 13.8;

IR ( $\mathrm{NaCl}$, thin film) 2959, 2928, 1682, 1449, 1366, $1326 \mathrm{~cm}^{-1}$;

HRMS (ESI-TOF) $\mathrm{m} / \mathrm{z}$ calcd for $\mathrm{C}_{18} \mathrm{H}_{26} \mathrm{~N}_{2} \mathrm{O}_{2} \mathrm{Na}(\mathrm{M}+\mathrm{Na})^{+}:$325.1886, found 325.1894. 

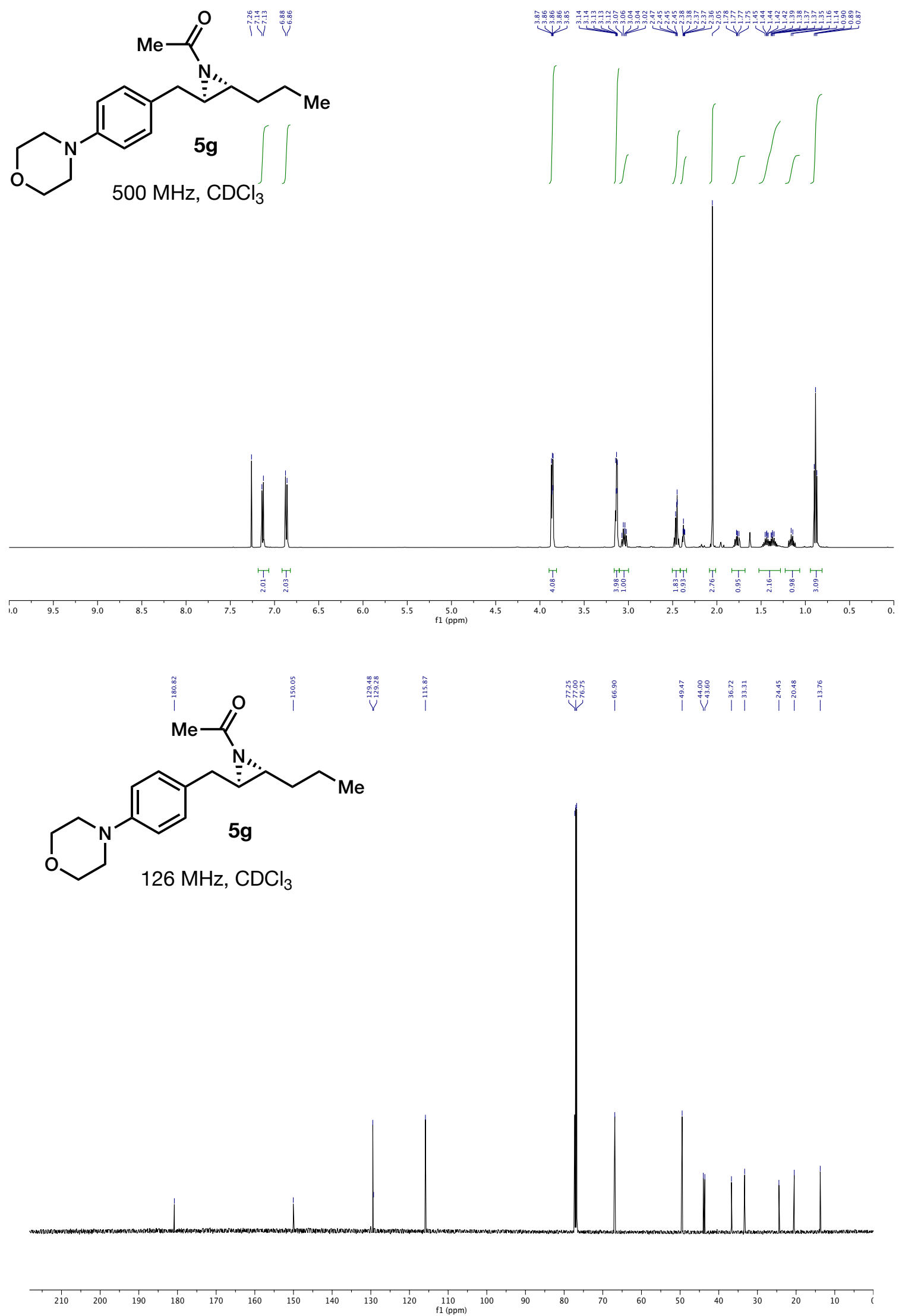


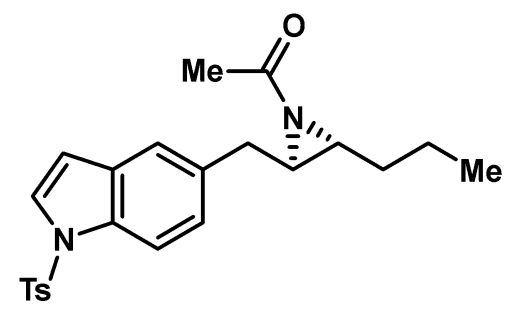

\section{1-((2R*,3R*)-2-Propyl-3-((1-tosyl-1H-indol-5-yl)methyl)aziridin-1-yl)ethan-1-one 5h}

General Procedure C: (E)-5-(Hex-2-en-1-yl)-1-tosyl-1H-indole (1.00 g, $2.83 \mathrm{mmol})$ was employed. Purification of the residue by column chromatography $(1: 1$, hexane:EtOAc) afforded the title compound $(0.94 \mathrm{~g}, 81 \%)$ as a yellow viscous oil.

${ }^{1} \mathrm{H}$ NMR $\left(400 \mathrm{MHz}, \mathrm{CDCl}_{3}\right) \delta 7.92(\mathrm{~d}, J=8.5 \mathrm{~Hz}, 1 \mathrm{H}), 7.74(\mathrm{~d}, J=8.4 \mathrm{~Hz}, 2 \mathrm{H}), 7.54(\mathrm{~d}, J=3.7$ $\mathrm{Hz}, 1 \mathrm{H}), 7.38(\mathrm{~d}, J=1.7 \mathrm{~Hz}, 1 \mathrm{H}), 7.23-7.13(\mathrm{~m}, 3 \mathrm{H}), 6.60(\mathrm{dd}, J=3.7,0.8 \mathrm{~Hz}, 1 \mathrm{H}), 3.14(\mathrm{dd}, J=$ 14.2, $5.0 \mathrm{~Hz}, 1 \mathrm{H}), 2.59$ (dd, $J=14.2,7.4 \mathrm{~Hz}, 1 \mathrm{H}), 2.51-2.44(\mathrm{~m}, 1 \mathrm{H}), 2.41-2.35(\mathrm{~m}, 1 \mathrm{H}), 2.32(\mathrm{~s}$, $3 \mathrm{H}), 2.00(\mathrm{~s}, 3 \mathrm{H}), 1.84-1.71(\mathrm{~m}, 1 \mathrm{H}), 1.47-1.22(\mathrm{~m}, 2 \mathrm{H}), 1.09-1.08(\mathrm{~m}, 1 \mathrm{H}), 0.83(\mathrm{t}, \mathrm{J}=7.4 \mathrm{~Hz}$, $3 \mathrm{H})$;

${ }^{13} \mathrm{C}$ NMR $\left(100 \mathrm{MHz}, \mathrm{CDCl}_{3}\right) \delta 180.7,144.9,135.2,133.7,133.0,133.0,131.1,129.8,126.7$, $125.4,121.2,113.5,108.9,43.9,43.6,37.4,33.1,24.3,21.5,20.4,13.6$;

IR (NaCl, thin film) 3052, 2960, 1681, 1460, 1370, $1267 \mathrm{~cm}^{-1}$;

HRMS (ESI-TOF) $m / z$ calcd for $\mathrm{C}_{23} \mathrm{H}_{26} \mathrm{~N}_{2} \mathrm{O}_{3} \mathrm{SNa}(\mathrm{M}+\mathrm{Na})^{+}$: 433.1556, found 433.1572; 

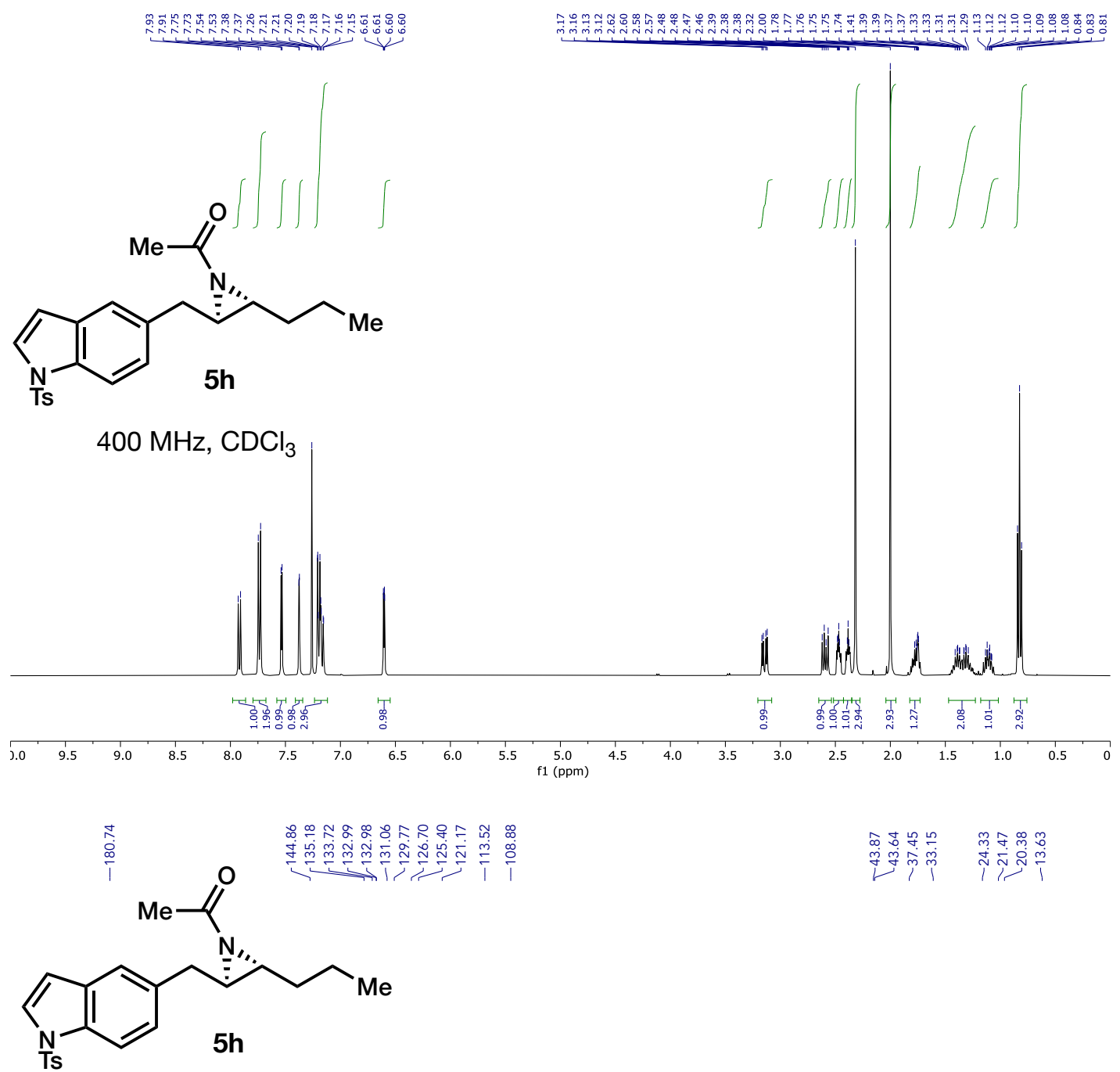

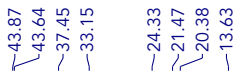

$100 \mathrm{MHz}, \mathrm{CDCl}_{3}$

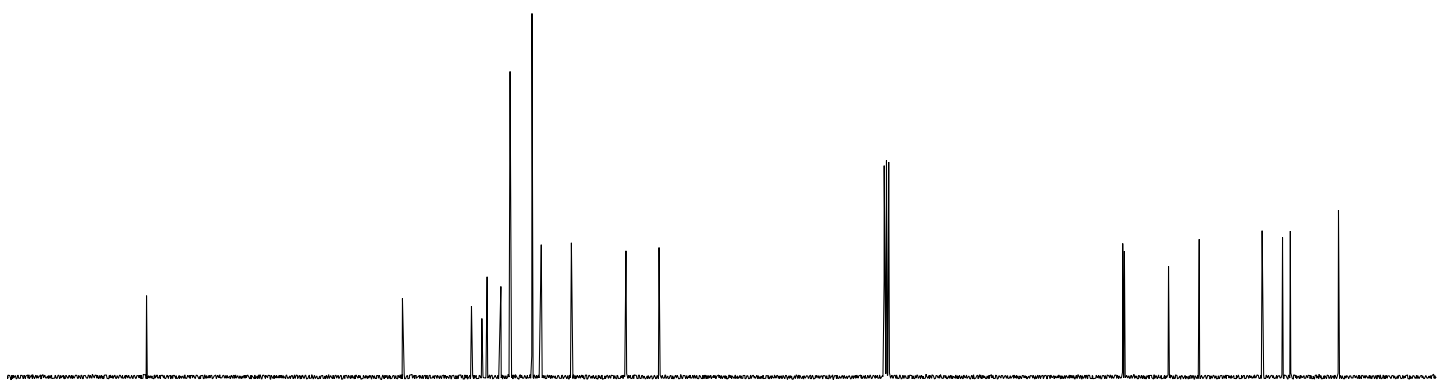

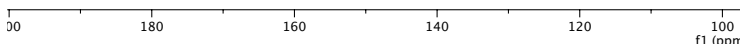


<smiles>CCCC1[C@H](Cc2ccc3c(c2)CCO3)N1C(C)=O</smiles>

1-((2R*,3R*)-2-((2,3-Dihydrobenzofuran-5-yl)methyl)-3-propylaziridin-1-yl)ethan-1-one 5i

General Procedure C: (E)-5-(Hex-2-en-1-yl)-2,3-dihydrobenzofuran (0.48 g, $2.38 \mathrm{mmol})$ was employed. Purification of the residue by column chromatography ( $3: 1$, hexane:EtOAc) afforded the title compound $(0.193 \mathrm{~g}, 31 \%)$ as a red oil.

${ }^{1} \mathrm{H}$ NMR $\left(400 \mathrm{MHz}, \mathrm{CDCl}_{3}\right) \delta 7.05(\mathrm{~s}, 1 \mathrm{H}), 6.97-6.90(\mathrm{~m}, 1 \mathrm{H}), 6.71(\mathrm{~d}, J=8.1 \mathrm{~Hz}, 1 \mathrm{H}), 4.55(\mathrm{t}, J=$ $8.7 \mathrm{~Hz}, 2 \mathrm{H}$ ), 3.18 (t, $J=8.7 \mathrm{~Hz}, 2 \mathrm{H}), 3.09-2.98(\mathrm{~m}, 1 \mathrm{H}), 2.55-2.40(\mathrm{~m}, 2 \mathrm{H}), 2.37$ (ddd, $J=7.6,5.0$, $2.5 \mathrm{~Hz}, 1 \mathrm{H}), 2.05(\mathrm{~s}, 3 \mathrm{H}), 1.85-1.73(\mathrm{~m}, 1 \mathrm{H}), 1.53-1.27(\mathrm{~m}, 2 \mathrm{H}), 1.21-1.08(\mathrm{~m}, 1 \mathrm{H}), 0.89(\mathrm{t}, J=7.4$ $\mathrm{Hz}, 3 \mathrm{H})$;

${ }^{13} \mathrm{C}$ NMR $\left(100 \mathrm{MHz}, \mathrm{CDCl}_{3}\right) \delta 180.8,158.9,129.7,128.2,127.2,125.3,109.4,71.2,44.2,43.6$, 37.1, 33.3, 29.7, 24.4, 20.4, 13.7;

IR ( $\mathrm{NaCl}$, thin film) 2959, 2929, 1683, 1492, 1366, $1244 \mathrm{~cm}^{-1}$;

HRMS (ESI-TOF) $\mathrm{m} / \mathrm{z}$ calcd for $\mathrm{C}_{16} \mathrm{H}_{21} \mathrm{NO}_{2} \mathrm{H}(\mathrm{M}+\mathrm{H})^{+}:$: 260.1645, found 260.1640. 
<smiles>C=CCC1C(NC(C)=O)[C@H]1Cc1ccc2c(c1)CCO2</smiles>

$400 \mathrm{MHz}, \mathrm{CDCl}_{3}$

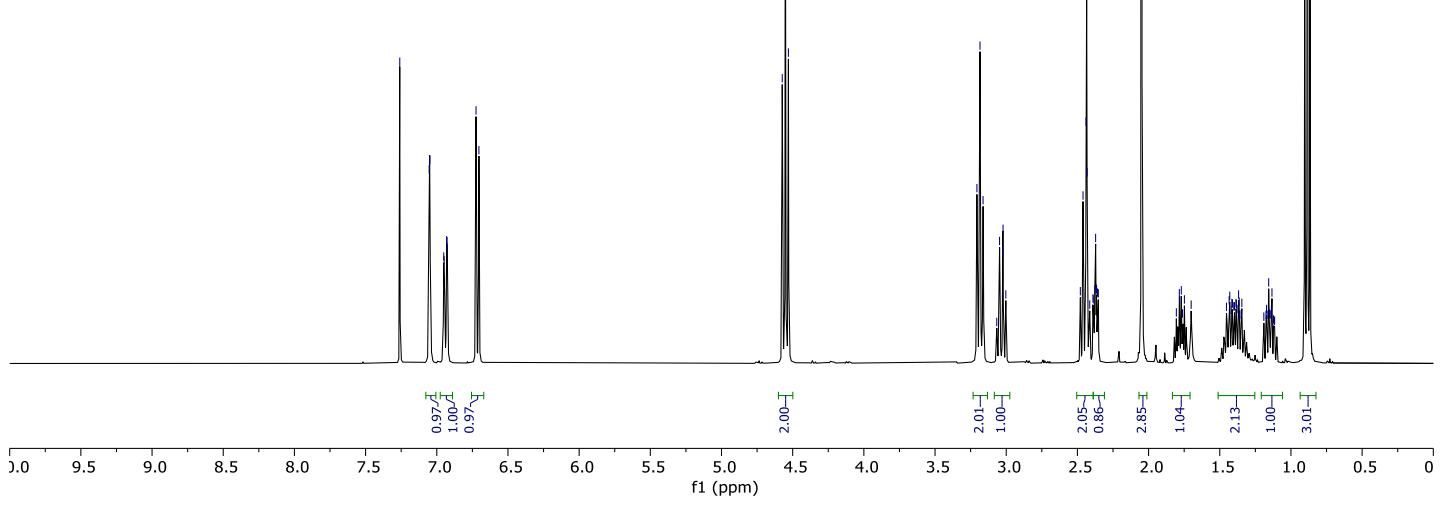

\begin{tabular}{|c|c|c|c|c|c|}
\hline$\stackrel{\substack{\infty \\
\infty}}{\stackrel{\infty}{\infty}}$ & 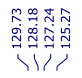 & 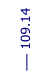 & 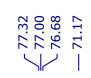 & 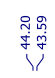 & 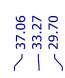 \\
\hline
\end{tabular}

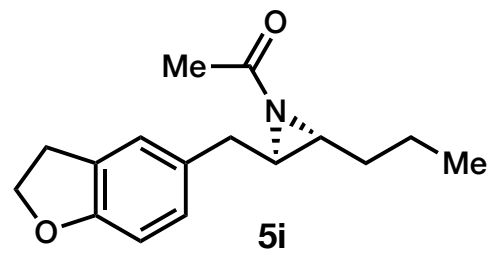

$100 \mathrm{MHz}, \mathrm{CDCl}_{3}$

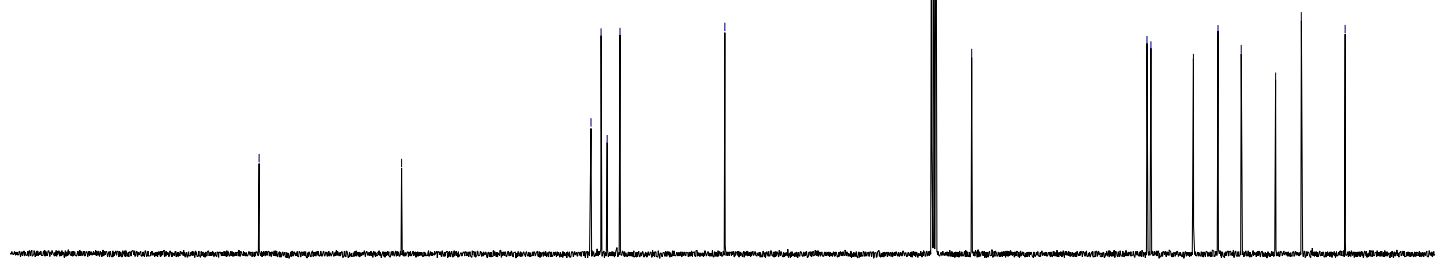




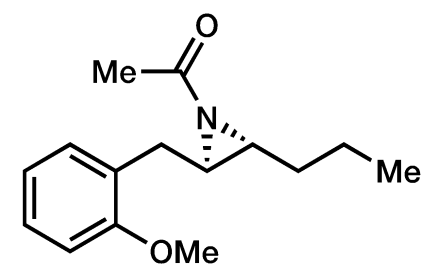

1-((2R* $\left.\left.3 R^{*}\right)-2-(2-M e t h o x y b e n z y l)-3-p r o p y l a z i r i d i n-1-y l\right) e t h a n-1-o n e ~ 5 j$

General Procedure C: (E)-1-(Hex-2-en-1-yl)-2-methoxybenzene $(1.70 \mathrm{~g}, 8.95 \mathrm{mmol})$ was employed. Purification of the residue by column chromatography $(0-15 \%$, ethyl acetate in hexanes) afforded the title compound (938 $\mathrm{mg}, 42 \%$ ) as a dark orange oil.

${ }^{1} \mathrm{H}$ NMR $\left(500 \mathrm{MHz}, \mathrm{CDCl}_{3}\right) \delta 7.23$ (td, $\left.J=7.8,1.7 \mathrm{~Hz}, 1 \mathrm{H}\right), 7.17(\mathrm{dd}, J=7.4,1.8 \mathrm{~Hz}, 1 \mathrm{H}), 6.90$ (dd, $J=7.4,1.1 \mathrm{~Hz}, 1 \mathrm{H}), 6.86(\mathrm{dd}, J=8.2,1.1 \mathrm{~Hz}, 1 \mathrm{H}), 3.83(\mathrm{~s}, 3 \mathrm{H}), 3.17-3.11(\mathrm{~m}, 1 \mathrm{H}), 2.55-2.50$ (m, 2H), 2.38 (ddd, $J=7.6,4.9,2.5 \mathrm{~Hz}, 1 \mathrm{H}), 2.05(\mathrm{~s}, 3 \mathrm{H}), 1.81-1.73(\mathrm{~m}, 1 \mathrm{H}), 1.44-1.26(\mathrm{~m}, 2 \mathrm{H})$, 1.13-1.06 (m, 1H), $0.84(\mathrm{t}, J=7.4 \mathrm{~Hz}, 3 \mathrm{H})$;

${ }^{13} \mathrm{C}$ NMR $\left(126 \mathrm{MHz}, \mathrm{CDCl}_{3}\right) \delta 180.9,157.4,130.4,128.0,126.3,120.5,110.2,55.1,43.7,43.0$, 33.3, 32.3, 24.4, 20.4, 13.6;

IR ( $\mathrm{NaCl}$, thin film) 2959, 2931, 1685, 1367, 1321, $1245 \mathrm{~cm}^{-1}$;

HRMS (ESI-TOF) $\mathrm{m} / \mathrm{z}$ calcd for $\mathrm{C}_{15} \mathrm{H}_{21} \mathrm{O}_{2} \mathrm{NNa}(\mathrm{M}+\mathrm{Na})^{+}: 270.1465$, found 270.1470 . 


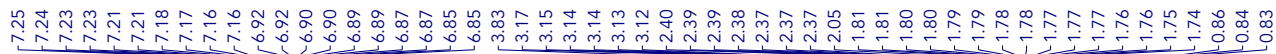

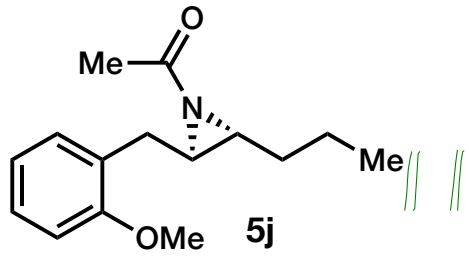

$500 \mathrm{MHz}, \mathrm{CDCl}_{3}$
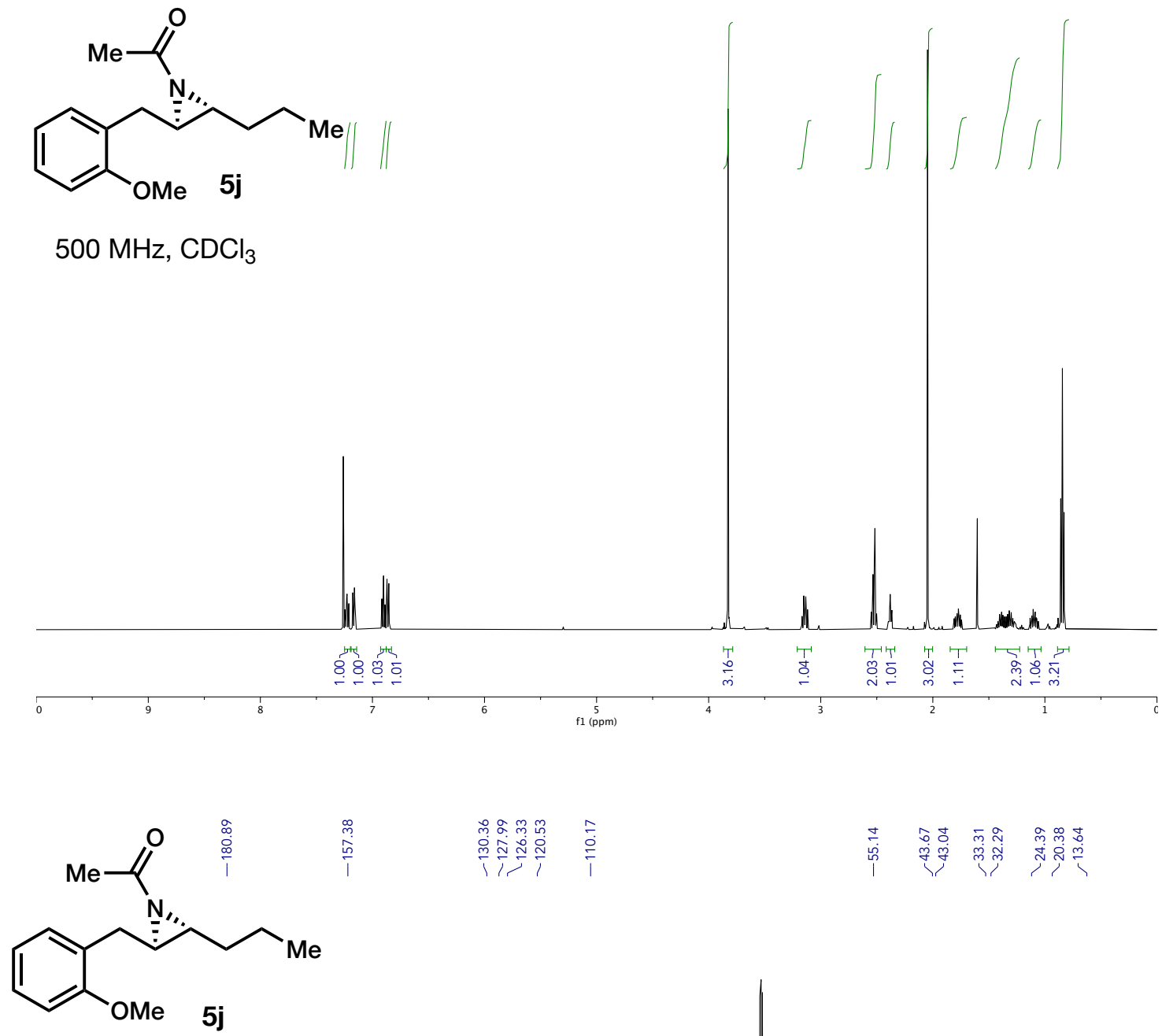

总

$126 \mathrm{MHz}, \mathrm{CDCl}_{3}$
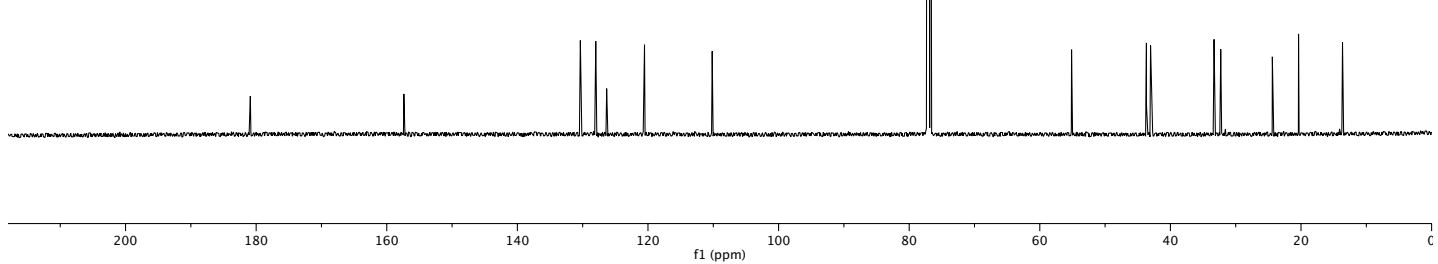
<smiles>COc1ccc(CC2[C@H](CC(C)C)N2C(C)=O)cc1</smiles>

\section{1-((2R*,3R*)-2-/sobutyl-3-(4-methoxybenzyl)aziridin-1-yl)ethan-1-one 5k}

General Procedure C: (E)-1-Methoxy-4-(5-methylhex-2-en-1-yl)benzene (1.35 g, 6.60 mmol) was employed. Purification of the residue by column chromatography (2:1, hexane:EtOAc) afforded the title compound $(0.95 \mathrm{~g}, 55 \%)$ as a red oil.

${ }^{1} \mathrm{H}$ NMR $\left(500 \mathrm{MHz}, \mathrm{CDCl}_{3}\right) \delta 7.14(\mathrm{~d}, J=8.6 \mathrm{~Hz}, 2 \mathrm{H}), 6.85(\mathrm{~d}, J=8.6 \mathrm{~Hz}, 2 \mathrm{H}), 3.79(\mathrm{~s}, 3 \mathrm{H}), 3.01$ (dd, $J=14.2,5.2 \mathrm{~Hz}, 1 \mathrm{H}$ ), $2.53(\mathrm{dd}, J=14.2,7.0 \mathrm{~Hz}, 1 \mathrm{H}), 2.48-2.41(\mathrm{~m}, 1 \mathrm{H}), 2.38$ (ddd, $J=8.3$, 4.7, $2.9 \mathrm{~Hz}, 1 \mathrm{H}$ ), $2.03(\mathrm{~s}, 3 \mathrm{H}), 1.77$ (ddd, $J=13.6,6.4,4.6 \mathrm{~Hz}, 1 \mathrm{H}), 1.74-1.61(\mathrm{~m}, 1 \mathrm{H}), 1.15-0.91$ $(\mathrm{m}, 1 \mathrm{H}), 0.89(\mathrm{~d}, J=6.6 \mathrm{~Hz}, 3 \mathrm{H}), 0.84(\mathrm{~d}, J=6.7 \mathrm{~Hz}, 3 \mathrm{H})$;

${ }^{13} \mathrm{C}$ NMR $\left(126 \mathrm{MHz} \mathrm{CDCl}_{3}\right) \delta 180.9,158.4,129.8,129.7,113.9,55.2,44.3,42.4,40.1,36.8,27.0$, 24.5, 22.6, 22.3;

IR (NaCl, thin film) 2956, 2930, 1685, 1442, 1366, $1302 \mathrm{~cm}^{-1}$;

HRMS (ESI-TOF) $\mathrm{m} / \mathrm{z}$ calcd for $\mathrm{C}_{16} \mathrm{H}_{23} \mathrm{NO}_{2} \mathrm{Na}(\mathrm{M}+\mathrm{Na})^{+}: 284.1610$, found 284.1619. 


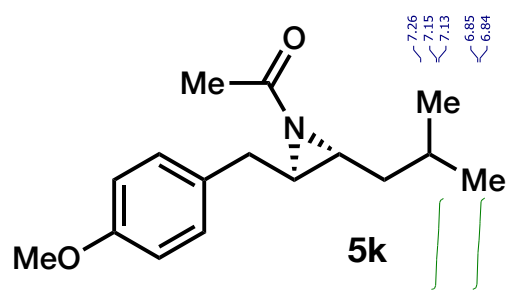

$500 \mathrm{MHz}, \mathrm{CDCl}_{3}$
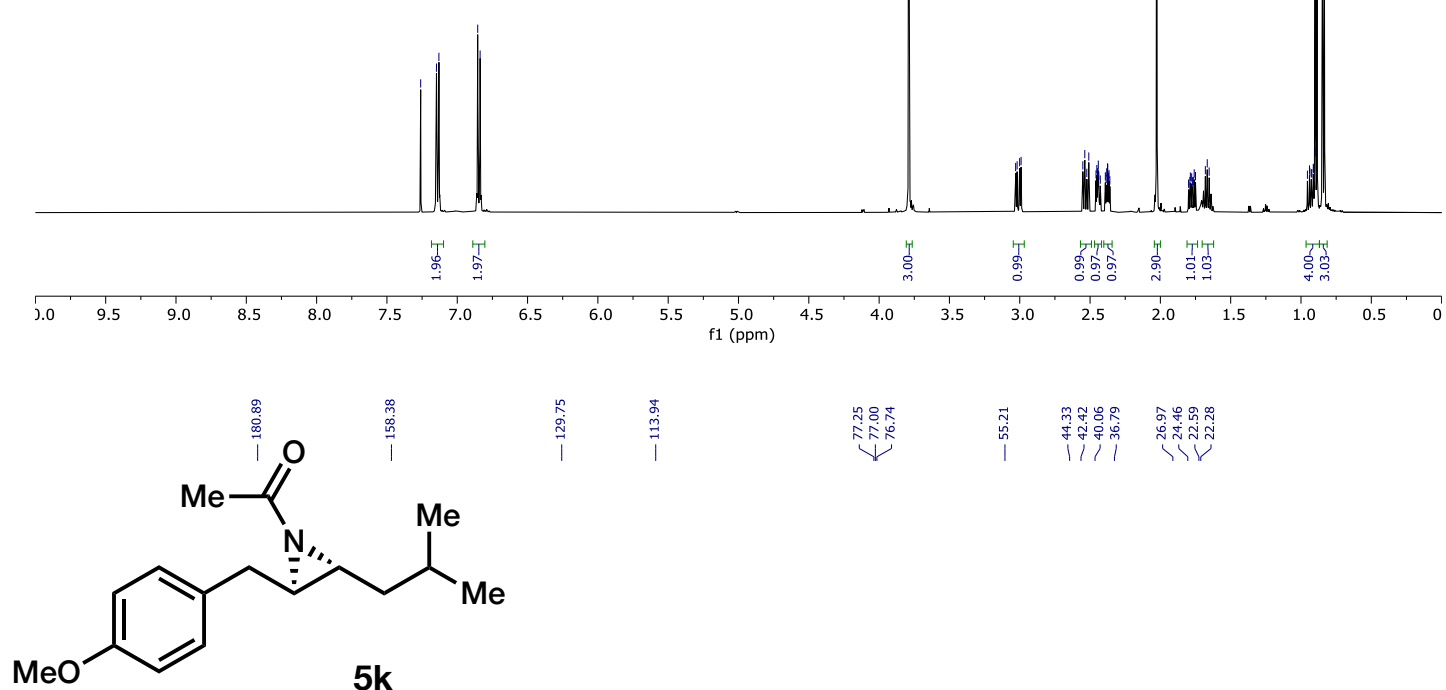

$126 \mathrm{MHz}, \mathrm{CDCl}_{3}$

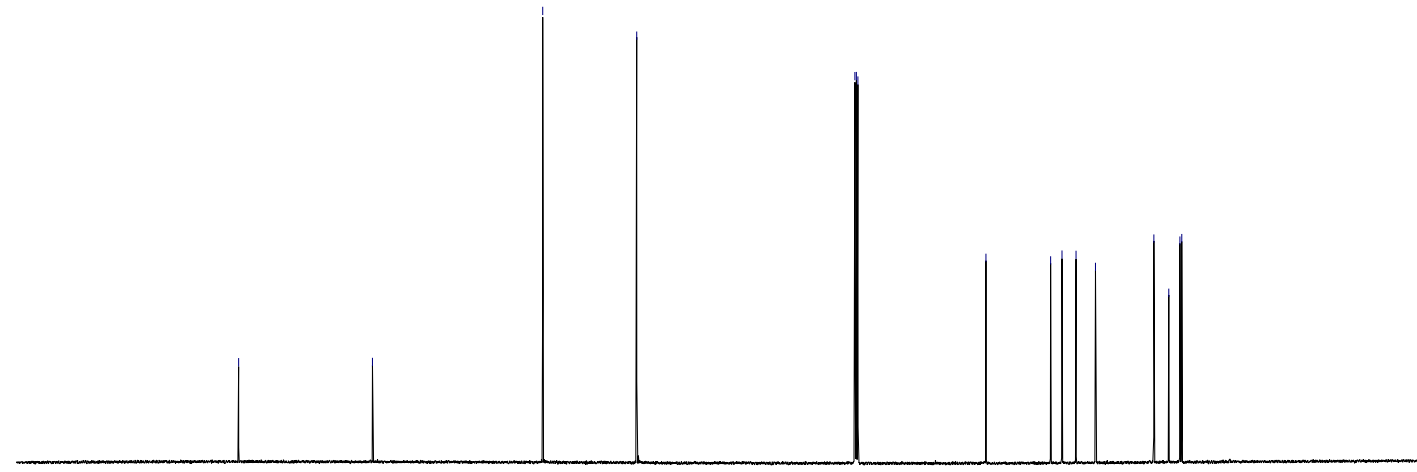

$\begin{array}{rllllllllllllllllllllllll}1 & 1 \\ 210 & 200 & 190 & 180 & 170 & 160 & 150 & 140 & 130 & 120 & 110 & 100 & 10 & 10 & 70 & 60 & 50 & 40 & 30 & 20 & 10 & 0 & -10 \\ \mathrm{f} 1(\mathrm{ppm})\end{array}$ 
<smiles>COc1ccc(CC2C([14CH3])[C@@H]2C(C)C)cc1</smiles>

\section{1-((2R*,3R*)-2-Isopropyl-3-(4-methoxybenzyl)aziridin-1-yl)ethan-1-one 5I}

General Procedure B: (E)-1-methoxy-4-(4-methylpent-2-en-1-yl)benzene (0.96 g, 5.05 mmol) was employed. Purification of the residue by column chromatography $(1: 1$, hexane:EtOAc) afforded the title compound $(0.42 \mathrm{~g}, 23 \%)$ as a red oil.

${ }^{1} \mathrm{H}$ NMR $\left(500 \mathrm{MHz}, \mathrm{CDCl}_{3}\right) \delta 7.82(\mathrm{~d}, \mathrm{~J}=8.3 \mathrm{~Hz}, 2 \mathrm{H}), 7.32-7.26(\mathrm{~m}, 2 \mathrm{H}), 7.10(\mathrm{~d}, J=8.6 \mathrm{~Hz}, 1 \mathrm{H})$, $6.81(\mathrm{~d}, J=8.6 \mathrm{~Hz}, 2 \mathrm{H}), 3.79(\mathrm{~s}, 4 \mathrm{H}), 3.24(\mathrm{dd}, J=14.1,4.4 \mathrm{~Hz}, 1 \mathrm{H}), 3.04(\mathrm{dd}, J=14.1,9.2 \mathrm{~Hz}$, $1 \mathrm{H}), 2.79-2.71(\mathrm{~m}, 1 \mathrm{H}), 2.66(\mathrm{dd}, J=8.4,4.6 \mathrm{~Hz}, 1 \mathrm{H}), 2.44(\mathrm{~s}, 3 \mathrm{H}), 1.56-1.46(\mathrm{~m}, 1 \mathrm{H}), 0.78$ (dd, $J=8.3,6.7 \mathrm{~Hz}, 6 \mathrm{H})$;

${ }^{13} \mathrm{C}$ NMR $\left(126 \mathrm{MHz}, \mathrm{CDCl}_{3}\right) \delta 158.4,143.8,137.7,130.1,129.8,129.4,127.6,114.0,55.3,55.2$, 50.2, 34.4, 29.9, 21.6, 20.3, 19.7;

IR ( $\mathrm{NaCl}$, thin film) 2961, 2932, 1611, 1513, 1320, $1303 \mathrm{~cm}^{-1}$;

HRMS (ESI-TOF) $\mathrm{m} / \mathrm{z}$ calcd for $\mathrm{C}_{20} \mathrm{H}_{25} \mathrm{NO}_{3} \mathrm{SNa}(\mathrm{M}+\mathrm{Na})^{+}:$382.1447, found 382.1449. 


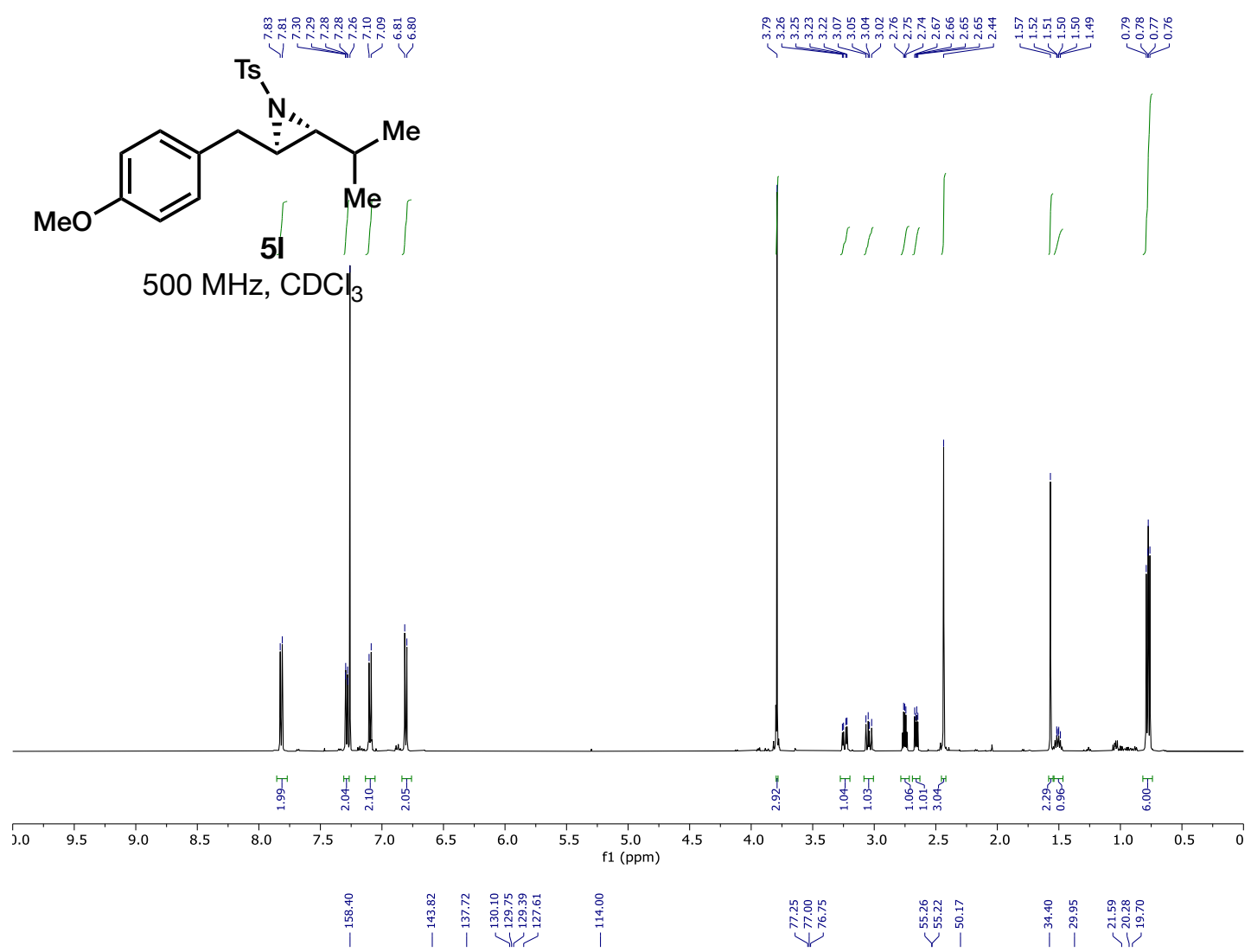<smiles>[3H]N[C@H]1C(Cc2ccc(OC)cc2)C1C(C)C</smiles>

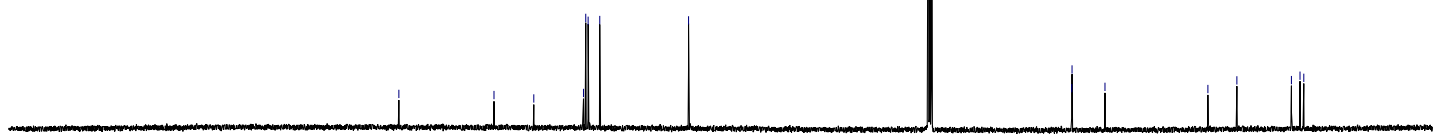


<smiles>COc1ccc(CC2C(CCCCCl)N2C(C)=O)cc1</smiles>

\section{1-((2R*,3R*)-2-(4-Chlorobutyl)-3-(4-methoxybenzyl)aziridin-1-yl)ethan-1-one 5m}

General Procedure C: (E)-1-(7-Chlorohept-2-en-1-yl)-4-methoxybenzene (1.00 g, $4.19 \mathrm{mmol})$ was employed. Purification of the residue by column chromatography (5-30\%, ethyl acetate in hexanes) afforded the title compound (651 mg, 53\%) as a dark orange oil.

${ }^{1} \mathrm{H}$ NMR $\left(500 \mathrm{MHz}, \mathrm{CDCl}_{3}\right) \delta 7.13(\mathrm{~d}, J=8.6 \mathrm{~Hz}, 2 \mathrm{H}), 6.86(\mathrm{~d}, J=8.6 \mathrm{~Hz}, 2 \mathrm{H}), 3.80(\mathrm{~s}, 3 \mathrm{H}), 3.46$ (t, $J=6.5 \mathrm{~Hz}, 2 \mathrm{H}), 3.11-3.05(\mathrm{~m}, 1 \mathrm{H}), 2.47-2.42(\mathrm{~m}, 2 \mathrm{H}), 2.37$ (ddd, J = 7.8, 5.3, $2.5 \mathrm{~Hz}, 1 \mathrm{H}), 2.05$ $(\mathrm{s}, 3 \mathrm{H}), 1.83-1.76(\mathrm{~m}, 1 \mathrm{H}), 1.73-1.67(\mathrm{~m}, 2 \mathrm{H}), 1.58-1.45(\mathrm{~m}, 2 \mathrm{H}), 1.27-1.19(\mathrm{~m}, 1 \mathrm{H})$;

${ }^{13} \mathrm{C}$ NMR $\left(126 \mathrm{MHz}, \mathrm{CDCl}_{3}\right) \delta 180.7,158.5,129.7,129.7,114.0,55.2,44.6,44.2,43.2,36.7,31.9$, $30.5,24.5,24.4$;

IR ( $\mathrm{NaCl}$, thin film) 2926, 1684, 1442, 1366, $1248 \mathrm{~cm}^{-1}$;

HRMS (ESI-TOF) $m / z$ calcd for $\mathrm{C}_{16} \mathrm{H}_{22} \mathrm{O}_{2} \mathrm{NCINa}(\mathrm{M}+\mathrm{Na})^{+}: 318.1237$, found 318.1232. 

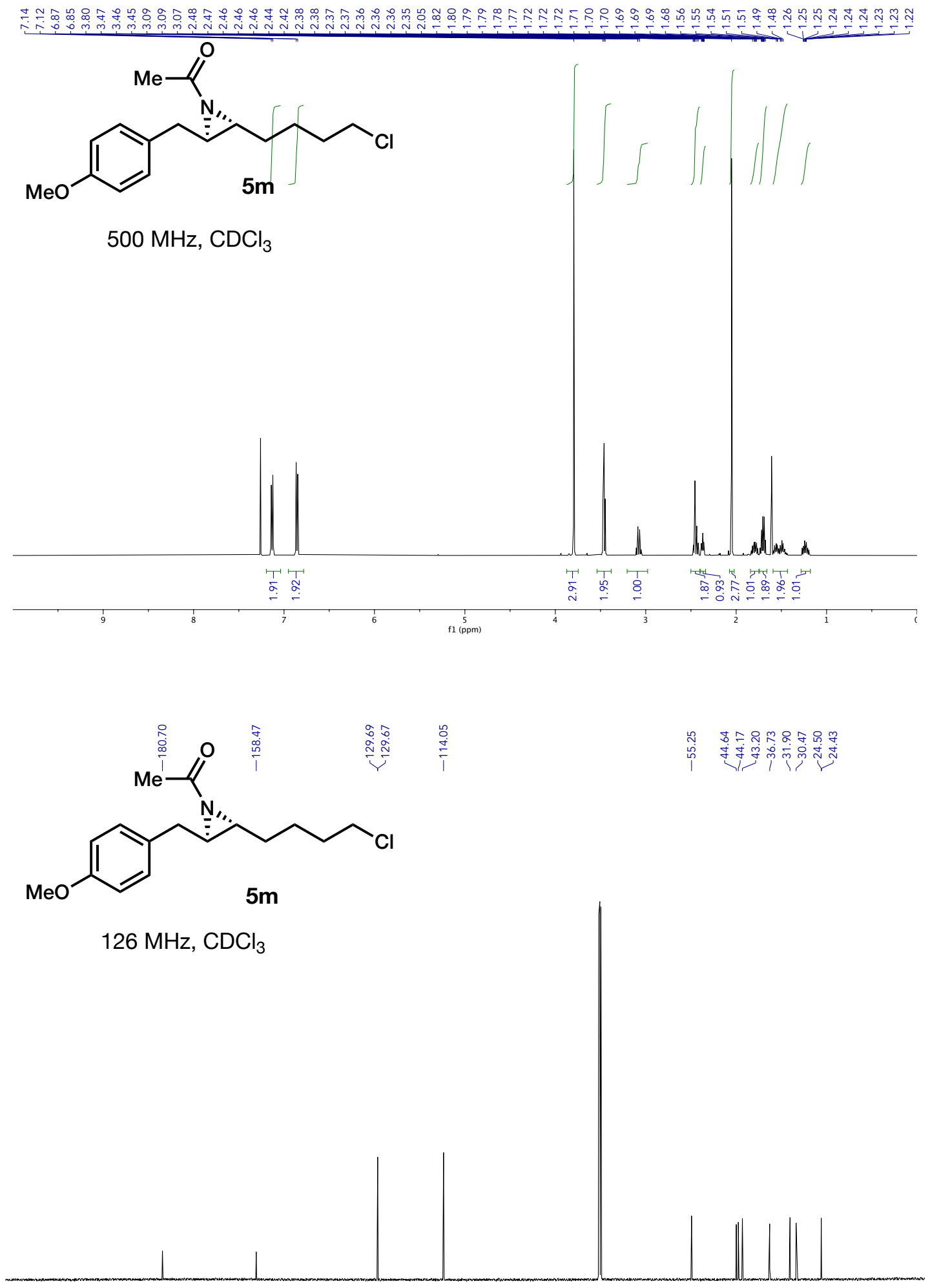
<smiles></smiles>

\section{5-((2R*,3R*)-1-Acetyl-3-(4-methoxybenzyl)aziridin-2-yl)pentanenitrile 5n}

General Procedure C: (E)-8-(4-Methoxyphenyl)oct-6-enenitrile (672 mg, $2.93 \mathrm{mmol}$ ) was employed. Purification of the residue by column chromatography (5-50\%, ethyl acetate in hexanes) afforded the title compound (373 mg, 44\%) as a dark orange oil.

${ }^{1} \mathrm{H}$ NMR $\left(500 \mathrm{MHz}, \mathrm{CDCl}_{3}\right) \delta 7.13(\mathrm{~d}, J=8.6 \mathrm{~Hz}, 2 \mathrm{H}), 6.87(\mathrm{~d}, J=8.7 \mathrm{~Hz}, 2 \mathrm{H}), 3.80(\mathrm{~s}, 3 \mathrm{H}), 3.09$ (dd, $J=13.6,4.3 \mathrm{~Hz}, 1 \mathrm{H}), 2.48-2.45(\mathrm{~m}, 1 \mathrm{H}), 2.41$ (dd, $J=13.6,7.9 \mathrm{~Hz}, 1 \mathrm{H}), 2.36$ (ddd, $J=7.1$, $5.5,2.8 \mathrm{~Hz}, 1 \mathrm{H}), 2.27(\mathrm{t}, J=6.8 \mathrm{~Hz}, 2 \mathrm{H}), 2.05(\mathrm{~s}, 3 \mathrm{H}), 1.80-1.74(\mathrm{~m}, 1 \mathrm{H}), 1.59-1.44(\mathrm{~m}, 4 \mathrm{H}), 1.32-$ $1.24(\mathrm{~m}, 1 \mathrm{H})$;

${ }^{13} \mathrm{C}$ NMR $\left(126 \mathrm{MHz}, \mathrm{CDCl}_{3}\right) \delta 180.7,158.5,129.6,129.6,119.4,114.1,55.3,44.22,42.8,36.7$, 30.4, 26.3, 24.7, 24.4, 17.0;

IR ( $\mathrm{NaCl}$, thin film) 2935, 2244, 1682, 1513, 1444, 1368, $1247 \mathrm{~cm}^{-1}$;

HRMS (ESI-TOF) $\mathrm{m} / \mathrm{z}$ calcd for $\mathrm{C}_{17} \mathrm{H}_{22} \mathrm{O}_{2} \mathrm{~N}_{2} \mathrm{Na}(\mathrm{M}+\mathrm{Na})^{+}: 309.1579$, found 309.1579. 


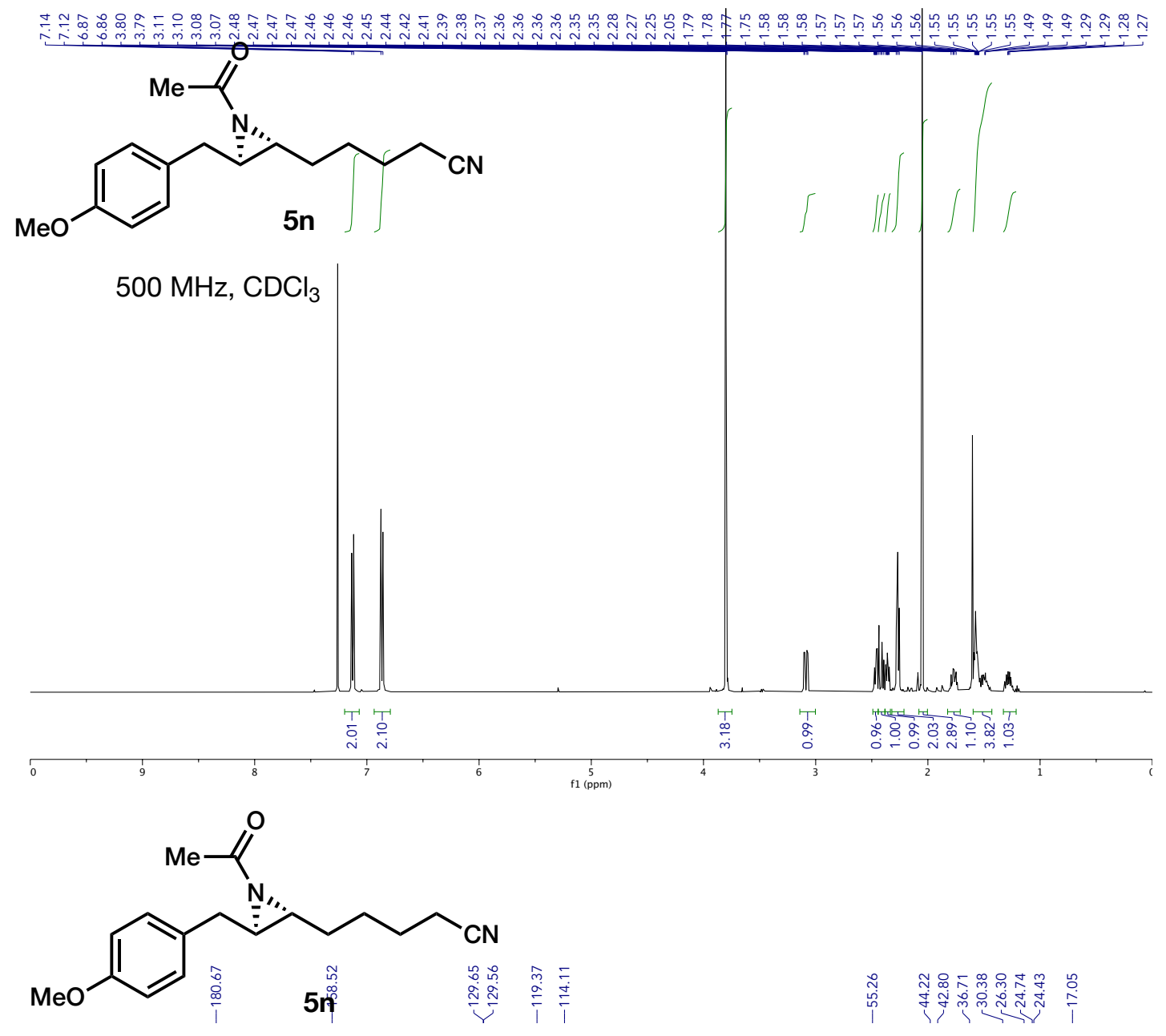

$126 \mathrm{MHz}, \mathrm{CDCl}_{3}$

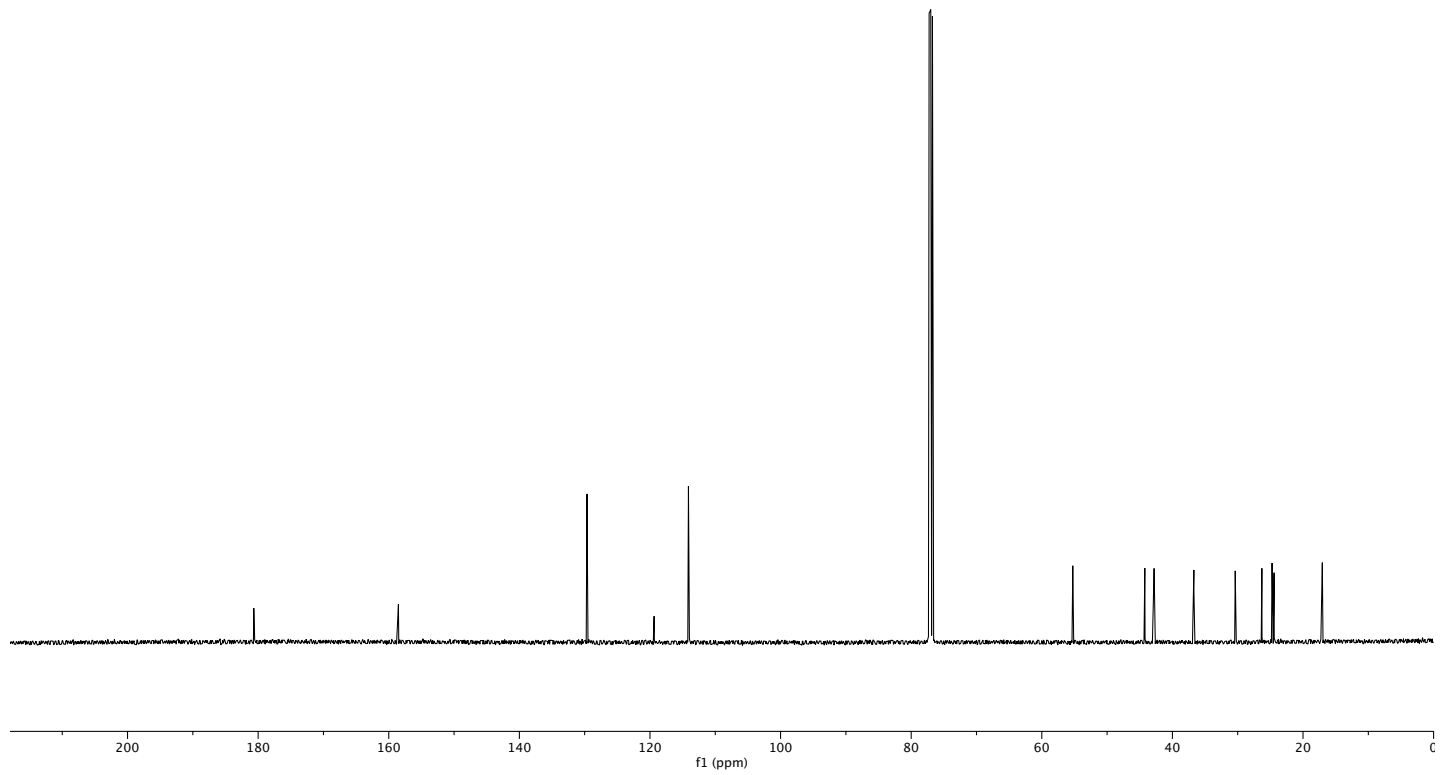


<smiles>COc1ccc(CC2[C@H](CCCCOCc3ccccc3)N2C(C)=O)cc1</smiles>

1-((2R*,3R*)-2-(4-(Benzyloxy)butyl)-3-(4-methoxybenzyl)aziridin-1-yl)ethan-1-one 50

General Procedure C: (E)-1-(7-(Benzyloxy)hept-2-en-1-yl)-4-methoxybenzene (1.00 g, 3.22 $\mathrm{mmol})$ was employed. Purification of the residue by column chromatography (1:1, hexane:EtOAc) afforded the title compound $(0.65 \mathrm{~g}, 55 \%)$ as a brown viscous oil.

${ }^{1} \mathrm{H}$ NMR $\left(500 \mathrm{MHz}, \mathrm{CDCl}_{3}\right) \delta$ 7.38-7.27 (m, 5H), $7.13(\mathrm{~d}, J=8.6 \mathrm{~Hz}, 2 \mathrm{H}), 6.84(\mathrm{~d}, J=8.6 \mathrm{~Hz}, 2 \mathrm{H})$, $4.47(\mathrm{~s}, 2 \mathrm{H}), 3.78(\mathrm{~s}, 3 \mathrm{H}), 3.41(\mathrm{t}, J=6.3 \mathrm{~Hz}, 2 \mathrm{H}), 3.05(\mathrm{dd}, J=13.1,3.8 \mathrm{~Hz}, 1 \mathrm{H}), 2.52-2.41(\mathrm{~m}$, $2 \mathrm{H}), 2.37(\mathrm{~m}, 1 \mathrm{H}), 2.03(\mathrm{~s}, 3 \mathrm{H}), 1.88-1.76(\mathrm{~m}, 1 \mathrm{H}), 1.63-1.53(\mathrm{~m}, 2 \mathrm{H}), 1.53-1.38(\mathrm{~m}, 2 \mathrm{H}), 1.24-$ $1.15(\mathrm{~m}, 1 \mathrm{H})$;

${ }^{13} \mathrm{C}$ NMR $\left(126 \mathrm{MHz}, \mathrm{CDCl}_{3}\right) \delta 180.8,158.4,138.5,129.8,129.7,128.4,127.6,127.5,114.0,72.9$, 69.9, 55.2, 44.1, 43.6, 36.7, 31.0, 29.3, 24.4, 23.9;

IR ( $\mathrm{NaCl}$, thin film) 3061, 2934, 1683, 1453, 1366, $1247 \mathrm{~cm}^{-1}$;

HRMS (ESI-TOF) $\mathrm{m} / z$ calcd for $\mathrm{C}_{23} \mathrm{H}_{29} \mathrm{NO}_{3} \mathrm{Na}(\mathrm{M}+\mathrm{Na})^{+}: 390.2040$, found 390.2059. 

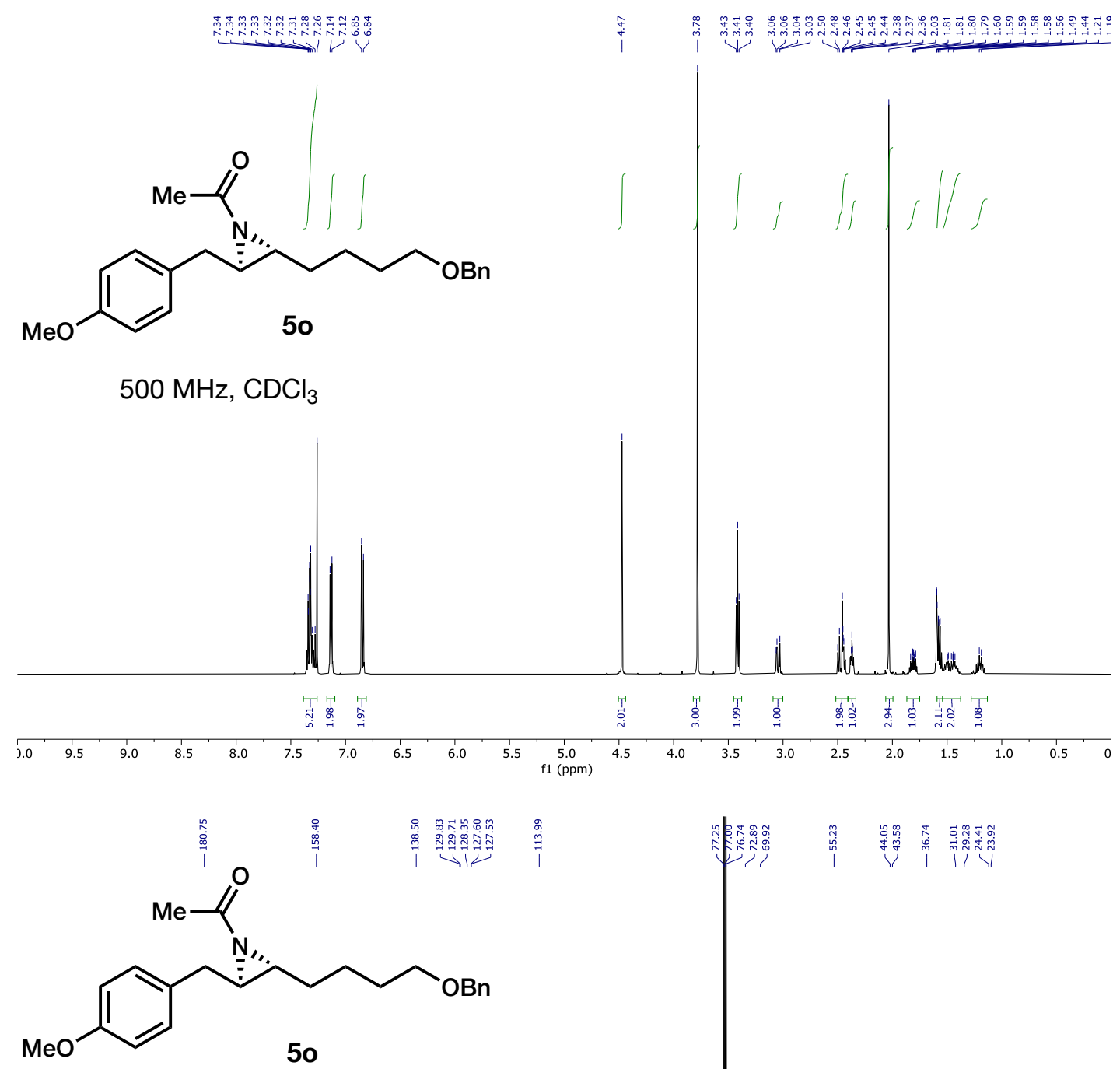

$126 \mathrm{MHz}, \mathrm{CDCl}_{3}$

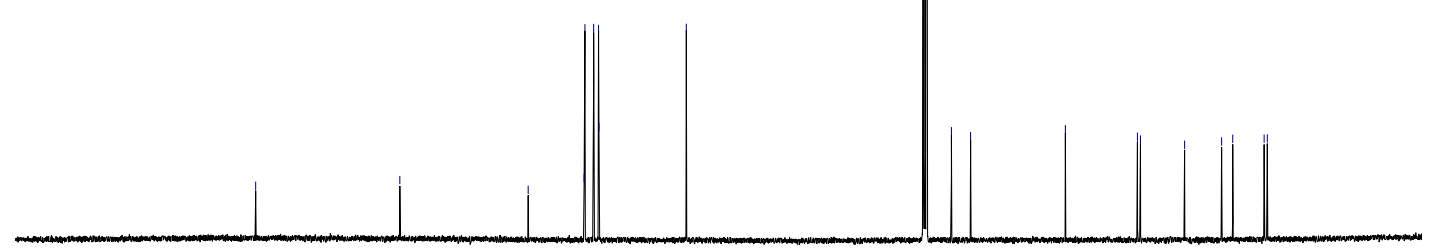

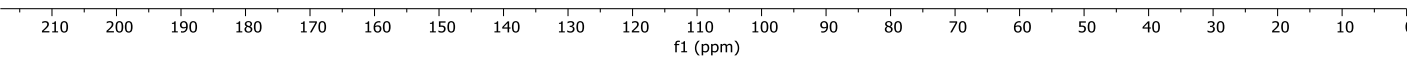


<smiles>COc1ccc(CC[C@@H]2C[C@H]2CCCCCN2C(=O)c3ccccc3C2=O)cc1</smiles>

\section{2-(4-((2R*,3R*)-1-Acetyl-3-(4-methoxybenzyl)aziridin-2-yl)butyl)isoindoline-1,3-dione 5p}

General Procedure C: (E)-2-(7-(4-Methoxyphenyl)hept-5-en-1-yl)isoindoline-1,3-dione (0.67 g, $1.92 \mathrm{mmol})$ was employed. Purification of the residue by column chromatography $(1: 1$, hexanesEtOAc) afforded the title compound $(0.61 \mathrm{~g}, 78 \%)$ as an orange viscous oil.

${ }^{1} \mathrm{H}$ NMR $\left(400 \mathrm{MHz}, \mathrm{CDCl}_{3}\right.$ ) $\delta 7.84$ (dd, $\left.J=5.5,3.1 \mathrm{~Hz}, 2 \mathrm{H}\right), 7.71$ (dd, $\left.J=5.5,3.1 \mathrm{~Hz}, 2 \mathrm{H}\right), 7.11$ $(\mathrm{d}, J=8.6 \mathrm{~Hz}, 2 \mathrm{H}), 6.82(\mathrm{~d}, J=8.6 \mathrm{~Hz}, 2 \mathrm{H}), 3.77(\mathrm{~s}, 3 \mathrm{H}), 3.62(\mathrm{t}, J=7.2 \mathrm{~Hz}, 2 \mathrm{H}), 3.05(\mathrm{q}, J=8.5$ $\mathrm{Hz}, 1 \mathrm{H}), 2.50-2.39(\mathrm{~m}, 2 \mathrm{H}), 2.39-2.31(\mathrm{~m}, 1 \mathrm{H}), 2.03(\mathrm{~s}, 3 \mathrm{H}), 1.90-1.78(\mathrm{~m}, 1 \mathrm{H}), 1.71-1.56(\mathrm{~m}$, $2 \mathrm{H}), 1.52-1.30(\mathrm{~m}, 2 \mathrm{H}), 1.24(\mathrm{dd}, J=8.1,4.1 \mathrm{~Hz}, 1 \mathrm{H})$;

${ }^{13} \mathrm{C}$ NMR $\left(100 \mathrm{MHz}_{\mathrm{CDCl}}\right) \delta 180.6,168.3,158.4,133.9,132.1,129.7,129.7,123.2,114.00$, $55.2,44.1,43.3,37.6,36.7,30.6,28.0,24.4,24.3$;

IR ( $\mathrm{NaCl}$, thin film) 2931, 2859, 1711, 1682, 1498, 1396, $1368 \mathrm{~cm}^{-1}$;

HRMS (ESI-TOF) $m / z$ calcd for $\mathrm{C}_{24} \mathrm{H}_{26} \mathrm{~N}_{2} \mathrm{O}_{4} \mathrm{H}(\mathrm{M}+\mathrm{H})^{+}$: 407.1965, found 407.1989; 

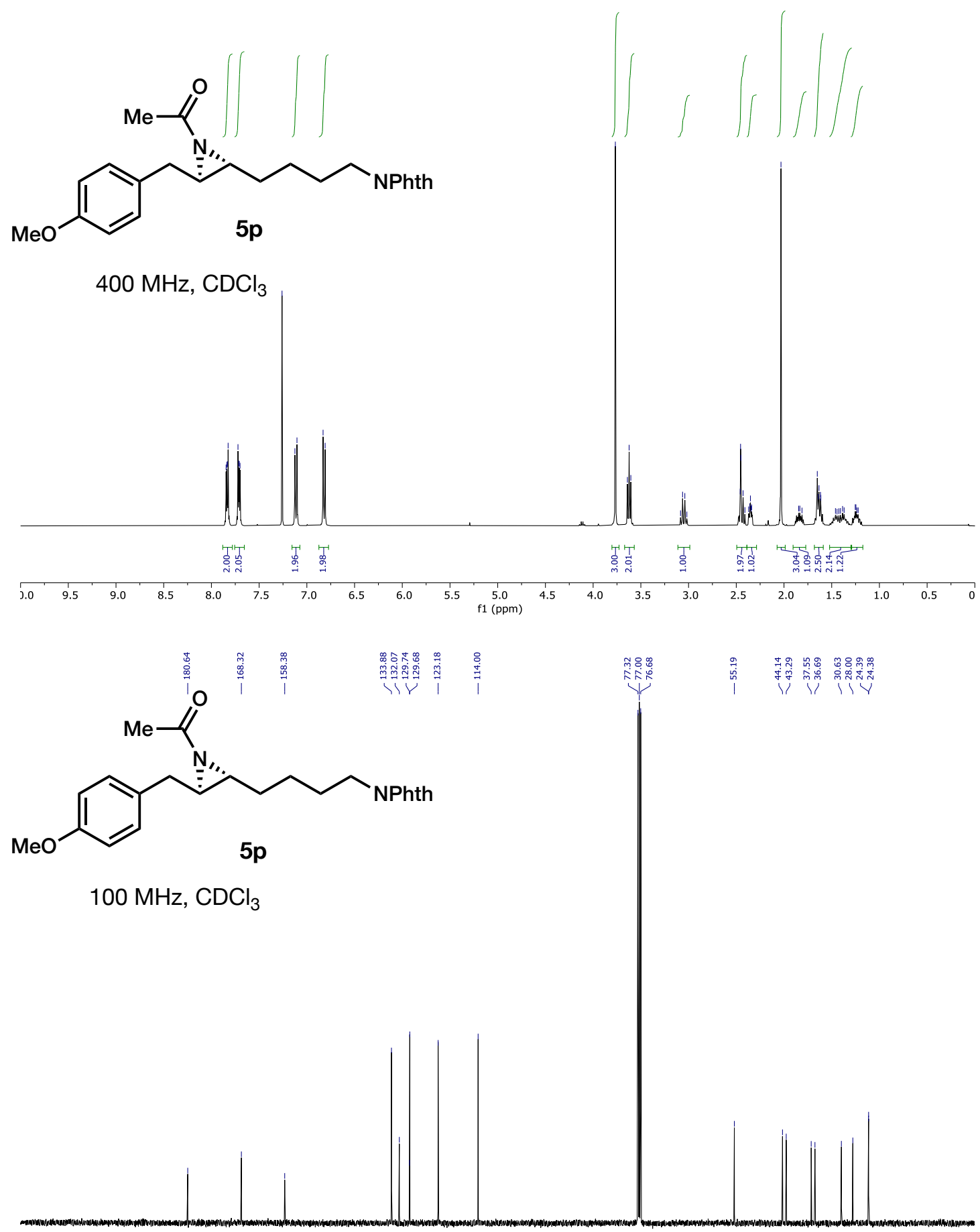
<smiles>COc1ccc(CC2C(CC3CC[N+](S)(S)CC3)N2C(C)=O)cc1</smiles>

1-((2R*, $\left.\left.3 R^{*}\right)-2-(4-M e t h o x y b e n z y l)-3-((1-t o s y l p i p e r i d i n-4-y l) m e t h y l) a z i r i d i n-1-y l\right) e t h a n-1-o n e$ $5 q$

General Procedure C: (E)-4-(4-(4-Methoxyphenyl)but-2-en-1-yl)-1-tosylpiperidine (1.53 g, 3.84 $\mathrm{mmol}$ ) was employed. Purification of the residue by column chromatography (5-65\%, ethyl acetate in hexanes) afforded the title compound $(1.01 \mathrm{~g}, 58 \%)$ as a dark orange oil.

${ }^{1} \mathrm{H}$ NMR $\left(500 \mathrm{MHz}, \mathrm{CDCl}_{3}\right) \delta 7.61(\mathrm{~d}, J=8.3 \mathrm{~Hz}, 2 \mathrm{H}), 7.32(\mathrm{~d}, J=8.2 \mathrm{~Hz}, 2 \mathrm{H}), 7.08(\mathrm{~d}, J=8.6$ $\mathrm{Hz}, 2 \mathrm{H}), 6.82(\mathrm{~d}, J=8.6 \mathrm{~Hz}, 2 \mathrm{H}), 3.79(\mathrm{~s}, 3 \mathrm{H}), 3.70-3.64(\mathrm{~m}, 2 \mathrm{H}), 3.05(\mathrm{dd}, J=13.6,4.6 \mathrm{~Hz}, 1 \mathrm{H})$, 2.44 (s, 3H), 2.42-2.40 (m, 1H), 2.35 (dd, $J=13.7,8.0 \mathrm{~Hz}, 1 \mathrm{H}), 2.31$ (ddd, $J=7.4,5.7,2.8 \mathrm{~Hz}$, $1 \mathrm{H}), 2.14-2.06(\mathrm{~m}, 2 \mathrm{H}), 2.02(\mathrm{~s}, 3 \mathrm{H}), 1.67-1.62(\mathrm{~m}, 2 \mathrm{H}), 1.58-1.54(\mathrm{~m}, 1 \mathrm{H}), 1.30-1.15(\mathrm{~m}, 3 \mathrm{H})$, 1.06 (app. dt, $J=14.0,7.0 \mathrm{~Hz}, 1 \mathrm{H}$ );

${ }^{13} \mathrm{C}$ NMR $\left(126 \mathrm{MHz}, \mathrm{CDCl}_{3}\right) \delta 180.8,158.5,143.4,133.0,129.6,129.5,129.4,127.7,114.0,55.2$, 46.2, 46.2, 44.5, 41.2, 37.5, 36.7, 34.1, 31.4, 30.9, 24.5, 21.5;

IR ( $\mathrm{NaCl}$, thin film) 2919, 2842, 1682, 1513, 1446, 1337, 1248, $1164 \mathrm{~cm}^{-1}$;

HRMS $\left(\mathrm{Cl}^{+}\right) \mathrm{m} / \mathrm{z}$ calcd for $\mathrm{C}_{25} \mathrm{H}_{32} \mathrm{O}_{4} \mathrm{~N}_{2} \mathrm{SH}(\mathrm{M}+\mathrm{H})^{+}: 457.2161$, found 457.2161. 

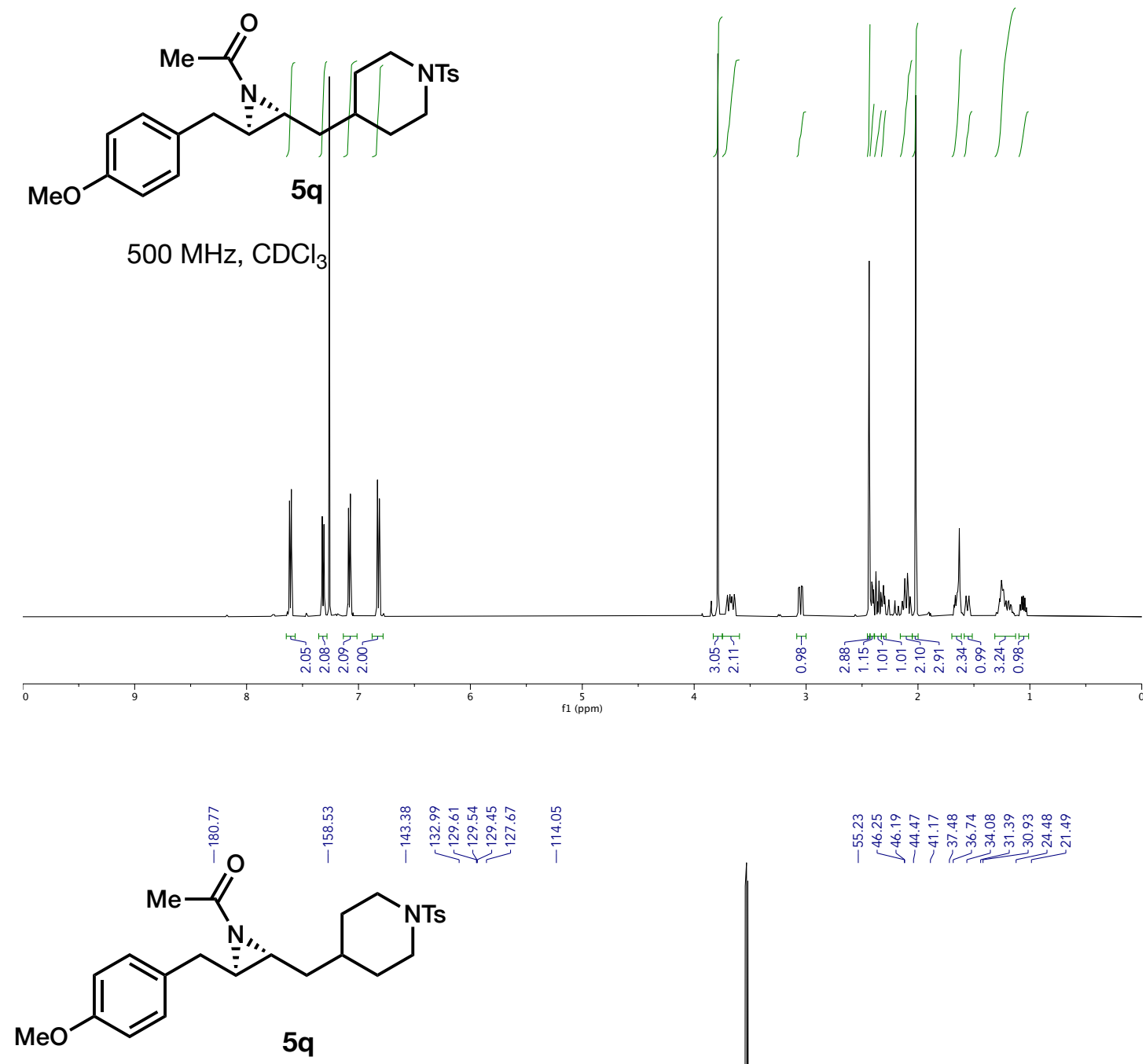

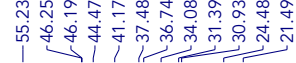

$126 \mathrm{MHz} \mathrm{CDCl}_{3}$

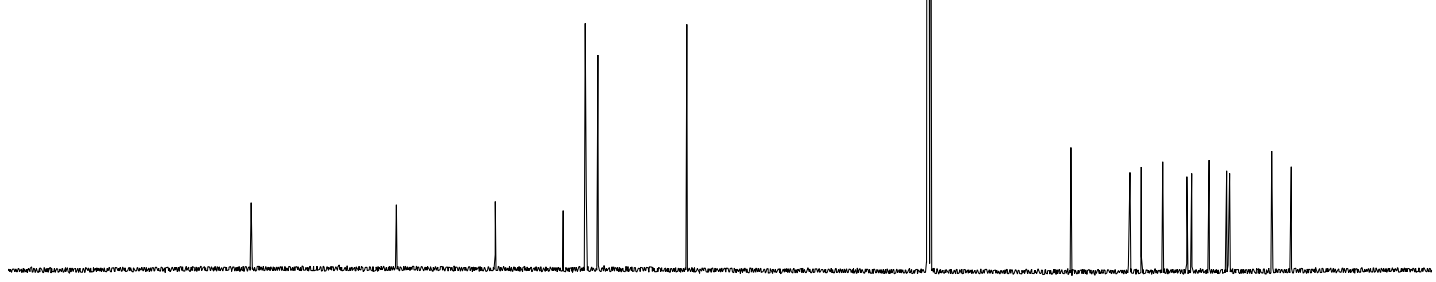

200

${ }_{180}^{1}$

160

140

120

100

80

60 


\section{Data for phenethylamine products}<smiles>CCCN[C@H](C(=O)O)[C@H](CCl)c1ccc(OC)cc1</smiles>

\section{$N$-((2R* $\left.3 R^{*}\right)$-1-Chloro-2-(4-methoxyphenyl)hexan-3-yl)acetamide 6a-Ac}

General Procedure D: Aziridine 5a-Ac $(74 \mathrm{mg}, 0.30 \mathrm{mmol})$ was employed. Purification of the residue by column chromatography (5-50\%, ethyl acetate in hexanes) afforded the title compound (72 $\mathrm{mg}, 85 \%)$ as an orange oil.

${ }^{1} \mathrm{H}$ NMR $\left(500 \mathrm{MHz}, \mathrm{CDCl}_{3}\right) \delta 7.11(\mathrm{~d}, J=8.7 \mathrm{~Hz}, 2 \mathrm{H}), 6.88(\mathrm{~d}, J=8.7 \mathrm{~Hz}, 2 \mathrm{H}), 5.22(\mathrm{~d}, J=9.6$ $\mathrm{Hz}, 1 \mathrm{H}), 4.29-4.23(\mathrm{~m}, 1 \mathrm{H}), 3.85$ (dd, $J=11.1,5.4 \mathrm{~Hz}, 1 \mathrm{H}), 3.81(\mathrm{~s}, 3 \mathrm{H}), 3.69$ (dd, $J=11.1,8.9$ $\mathrm{Hz}, 1 \mathrm{H}), 2.91(\mathrm{app} t \mathrm{td}, J=8.8,5.4 \mathrm{~Hz}, 1 \mathrm{H}), 2.03(\mathrm{~s}, 3 \mathrm{H}), 1.39-1.28(\mathrm{~m}, 2 \mathrm{H}), 1.23-1.16(\mathrm{~m}, 1 \mathrm{H})$, $1.12-1.06(\mathrm{~m}, 1 \mathrm{H}), 0.80(\mathrm{t}, J=7.1 \mathrm{~Hz}, 3 \mathrm{H})$;

${ }^{13} \mathrm{C}$ NMR $\left(126 \mathrm{MHz}, \mathrm{CDCl}_{3}\right) \delta 170.0,158.8,131.2,129.3,114.1,55.2,52.9,51.7,47.0,34.3,23.5$, 18.9, 13.7;

IR ( $\mathrm{NaCl}$, thin film) 3269, 2958, 1645, 1514, $1249 \mathrm{~cm}^{-1}$;

HRMS (ESI-TOF) $\mathrm{m} / \mathrm{z}$ calcd for $\mathrm{C}_{15} \mathrm{H}_{22} \mathrm{O}_{2} \mathrm{NCINa}(\mathrm{M}+\mathrm{Na})^{+}: 306.1231$, found 306.1233. 


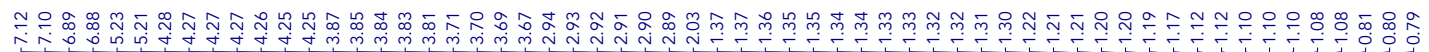
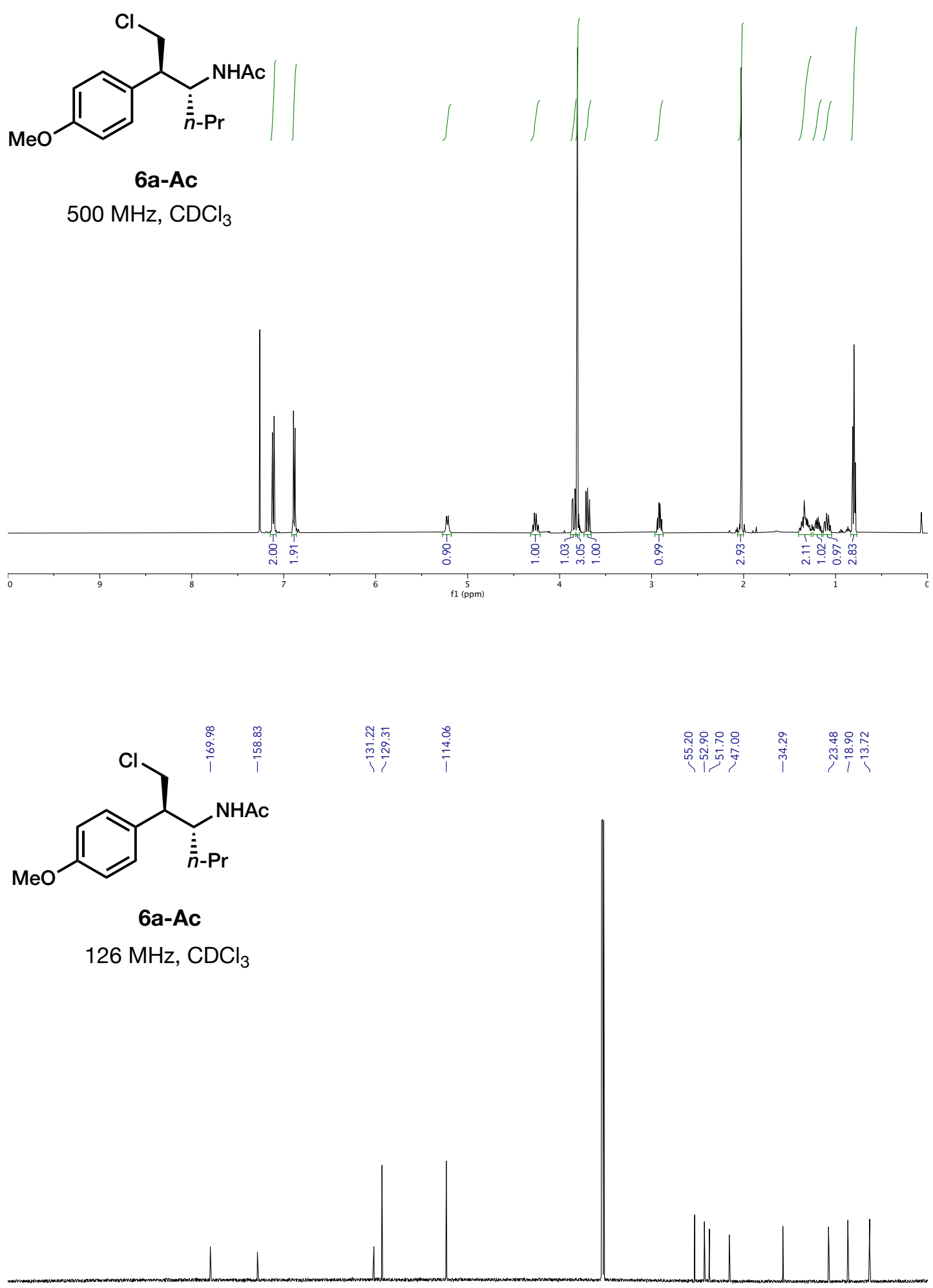
<smiles>CCCCC(N)[C@H](CCl)c1ccc(OC)cc1</smiles>

$N-\left(\left(2 R^{*}, 3 R^{*}\right)-1-C h l o r o-2-(4-m e t h o x y p h e n y l) h e x a n-3-y l\right)-4-m e t h y l b e n z e n e s u l f o n a m i d e$ 6a-Ts

General Procedure D: Aziridine 5a-Ts $(108 \mathrm{mg}, 0.30 \mathrm{mmol})$ was employed. Purification of the residue by column chromatography $(5-15 \%$, ethyl acetate in hexanes) afforded the title compound (74 $\mathrm{mg}, 62 \%)$ as a white solid.

${ }^{1} \mathrm{H}$ NMR $\left(500 \mathrm{MHz}, \mathrm{CDCl}_{3}\right) \delta 7.79(\mathrm{~d}, J=8.3 \mathrm{~Hz}, 2 \mathrm{H}), 6.33(\mathrm{~d}, J=7.3 \mathrm{~Hz}, 2 \mathrm{H}), 6.97(\mathrm{~d}, J=8.7$ $\mathrm{Hz}, 2 \mathrm{H}), 6.84(\mathrm{~d}, J=8.7 \mathrm{~Hz}, 2 \mathrm{H}), 4.20(\mathrm{~d}, J=9.6 \mathrm{~Hz}, 1 \mathrm{H}), 3.84(\mathrm{dd}, J=11.1,7.9 \mathrm{~Hz}, 1 \mathrm{H}), 3.79$ (s, 3H), 3.74-3.68 (m, 1H), $3.62(\mathrm{dd}, J=11.2,7.5 \mathrm{~Hz}, 1 \mathrm{H}), 2.97$ (app td, J=7.7, $6.4 \mathrm{~Hz}, 1 \mathrm{H}), 2.44$ $(\mathrm{s}, 3 \mathrm{H}), 1.35-1.29(\mathrm{~m}, 1 \mathrm{H}), 1.24-1.16(\mathrm{~m}, 1 \mathrm{H}), 1.03-0.93(\mathrm{~m}, 2 \mathrm{H}), 0.68(\mathrm{t}, J=7.1 \mathrm{~Hz}, 3 \mathrm{H})$;

${ }^{13} \mathrm{C}$ NMR $\left(126 \mathrm{MHz}, \mathrm{CDCl}_{3}\right) \delta 159.0,143.5,138.1,129.7,129.7,129.5,127.1,114.1,55.2,55.2$, $51.3,45.9,32.8,21.5,18.2,13.6$;

IR ( $\mathrm{NaCl}$, thin film) 3422, 2959, 1611, 1514, 1328, 1250, $1158 \mathrm{~cm}^{-1}$;

HRMS $\left(\mathrm{Cl}^{+}\right) \mathrm{m} / \mathrm{z}$ calcd for $\mathrm{C}_{20} \mathrm{H}_{26} \mathrm{O}_{3} \mathrm{NCISNH}_{4}\left(\mathrm{M}+\mathrm{NH}_{4}\right)^{+}:$413.1660, found 413.1643;

m. pt $118-121^{\circ} \mathrm{C}$ 

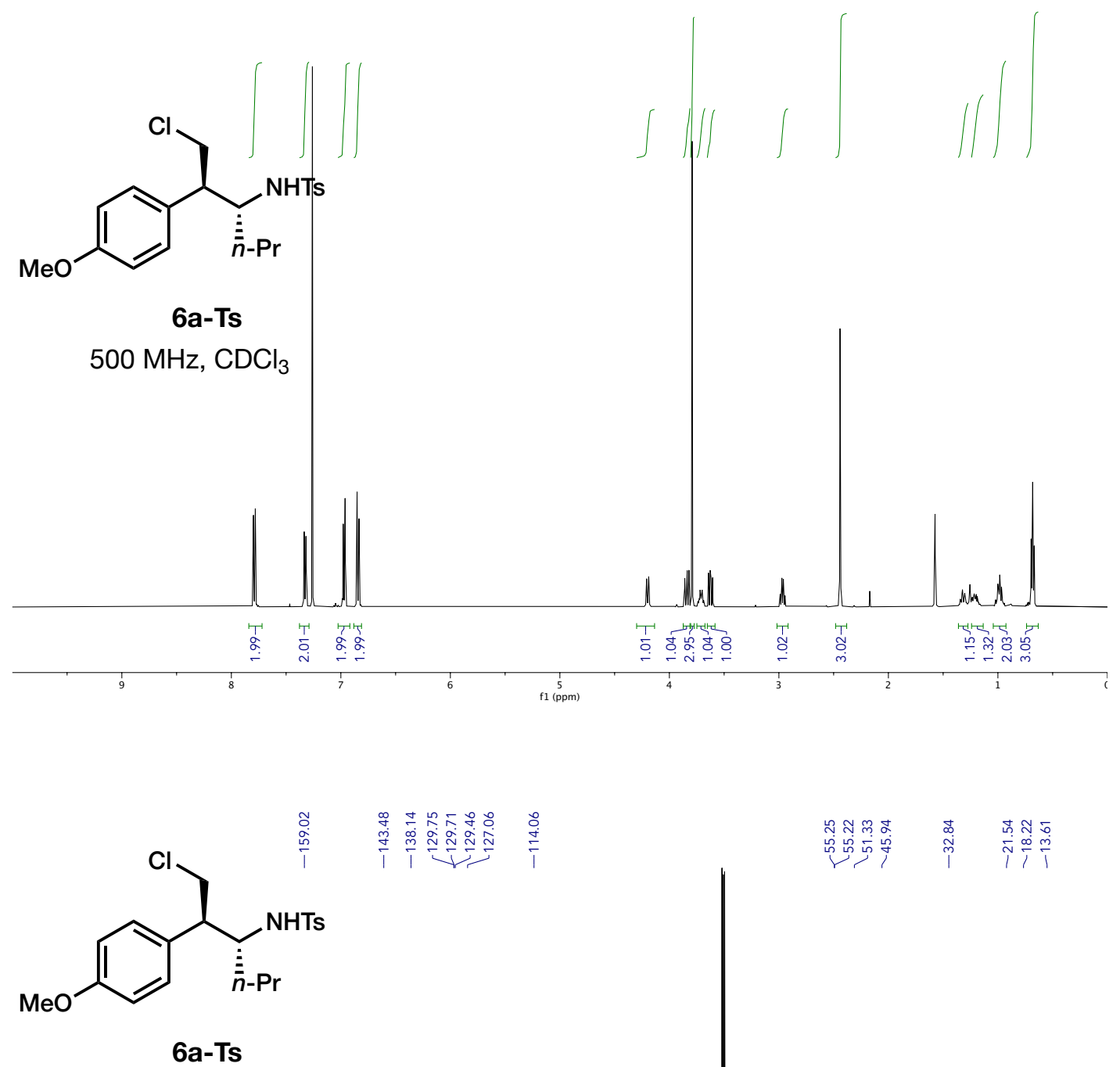

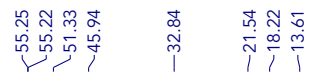

$126 \mathrm{MHz}, \mathrm{CDCl}_{3}$

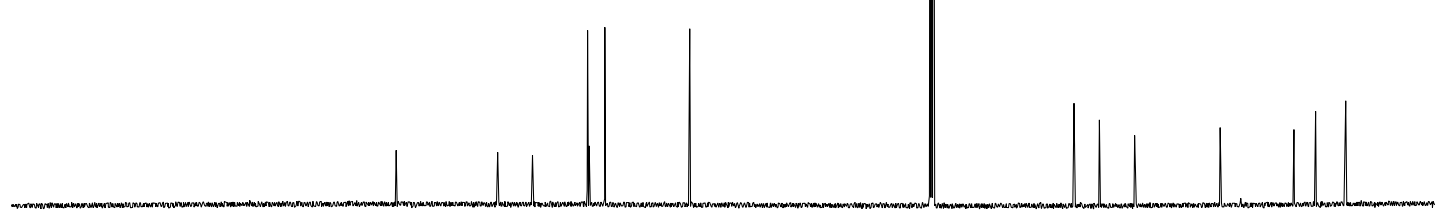

200

180

160

140

$120 \quad \mathrm{f} 1(\mathrm{ppm}) \quad 100$

80

60 
<smiles>CCCCC(NC)[C@H](CCl)c1ccccc1</smiles>

\section{$N-\left(\left(2 R^{*}, 3 R^{*}\right)-1-C h l o r o-2-p h e n y l h e x a n-3-y l\right)$ acetamide $6 \mathrm{~b}$}

To a solution of $\mathrm{TiCl}_{4}$ in dichloromethane $(1 \mathrm{~mL}, 0.15 \mathrm{M})$ was added aziridine $5 \mathrm{~b}-\mathrm{Ac}(65 \mathrm{mg}, 0.30$ $\mathrm{mmol})$ in dichloromethane $(2 \mathrm{~mL})$ dropwise. The mixture was stirred at room temperature for 10 minutes. The reaction was quenched with $\mathrm{NaHCO}_{3}(0.5 \mathrm{~mL})$, filtered through Celite $\circledast$, and extracted with dichloromethane $(3 \times 5 \mathrm{~mL})$. The combined organic layers were washed with $\mathrm{NaHCO}_{3}(5 \mathrm{~mL})$ and brine $(5 \mathrm{~mL})$, dried $\left(\mathrm{MgSO}_{4}\right)$, and concentrated in vacuo. Purification of the residue by column chromatography $(10-55 \%$, ethyl acetate in hexanes) afforded the title compound (41 mg, $54 \%$ ) as a white solid.

${ }^{1} \mathrm{H}$ NMR $\left(500 \mathrm{MHz}, \mathrm{CDCl}_{3}\right) \delta 7.35(\mathrm{dd}, J=8.0,6.5 \mathrm{~Hz}, 2 \mathrm{H}), 7.30-7.27(\mathrm{~m}, 1 \mathrm{H}), 7.21-7.19(\mathrm{~m}, 2 \mathrm{H})$, 5.50 (br. s, $1 \mathrm{H}$ ), 4.34-4.28 (m, 1H), 3.87 (dd, $J=11.1,5.3 \mathrm{~Hz}, 1 \mathrm{H}), 3.74(\mathrm{dd}, J=11.1,8.8 \mathrm{~Hz}$, $1 \mathrm{H}), 2.97(\mathrm{app} \mathrm{td}, J=8.9,5.3 \mathrm{~Hz}, 1 \mathrm{H}), 2.04(\mathrm{~s}, 3 \mathrm{H}), 1.37-1.28(\mathrm{~m}, 2 \mathrm{H}), 1.23-1.17(\mathrm{~m}, 1 \mathrm{H}), 1.15-$ $1.08(\mathrm{~m}, 1 \mathrm{H}), 0.79(\mathrm{t}, J=6.9 \mathrm{~Hz}, 3 \mathrm{H})$;

${ }^{13} \mathrm{C}$ NMR $\left(126 \mathrm{MHz}_{\mathrm{CDCl}}\right) \delta$ 170.2, 139.3, 128.7, 128.3, 127.4, 53.7, 51.7, 46.8, 34.3, 23.4, 18.9, 13.7

IR ( $\mathrm{NaCl}$, thin film) 3268, 2958, 1645, 1552, $1374 \mathrm{~cm}^{-1}$;

HRMS (ESI-TOF) $\mathrm{m} / \mathrm{z}$ calcd for $\mathrm{C}_{14} \mathrm{H}_{20} \mathrm{ONCINa}(\mathrm{M}+\mathrm{Na})^{+}:$276.1126, found 276.1122;

m. pt $102-106^{\circ} \mathrm{C}$ 

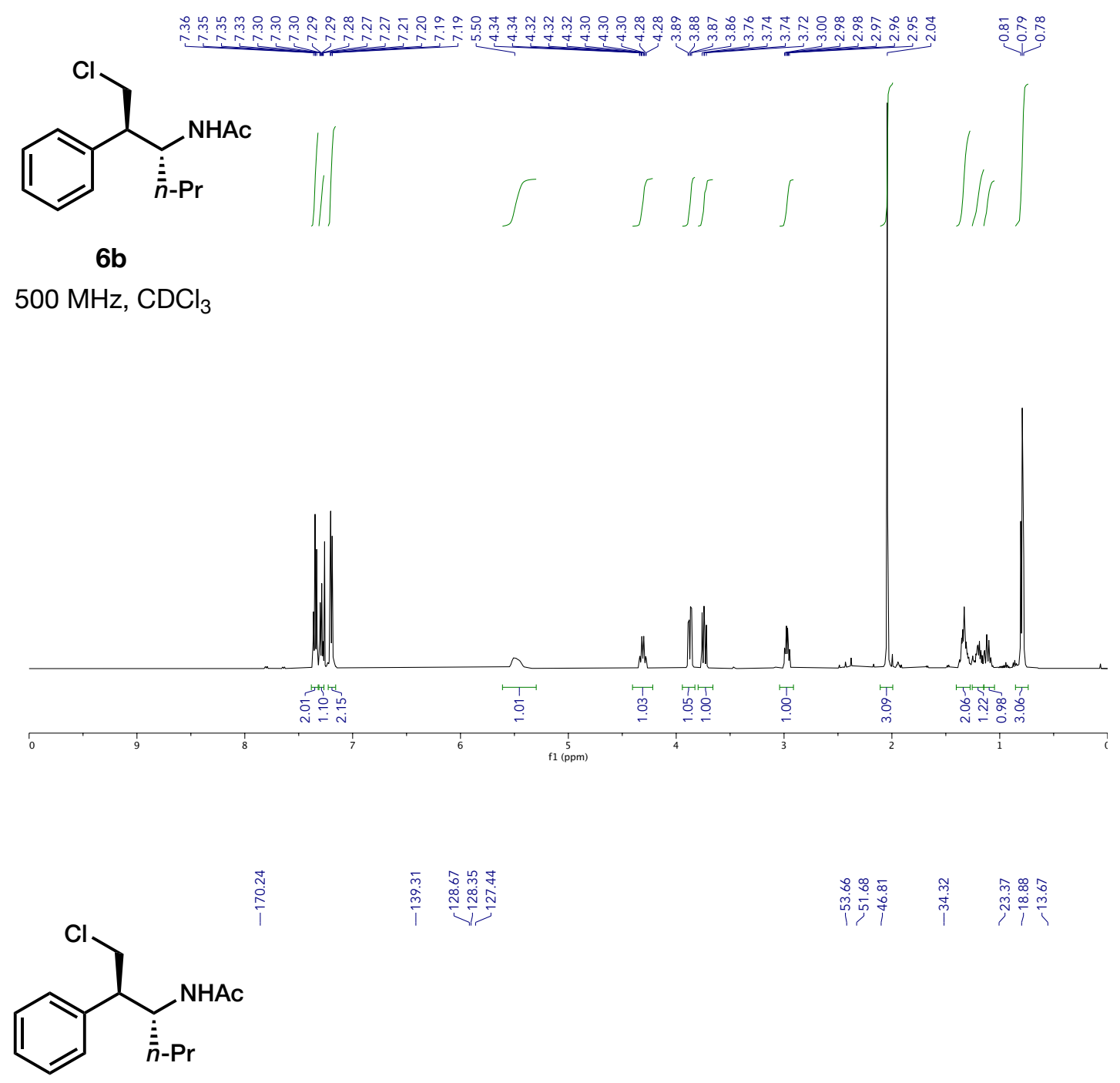

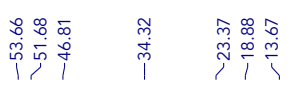

6b

$126 \mathrm{MHz}, \mathrm{CDCl}_{3}$

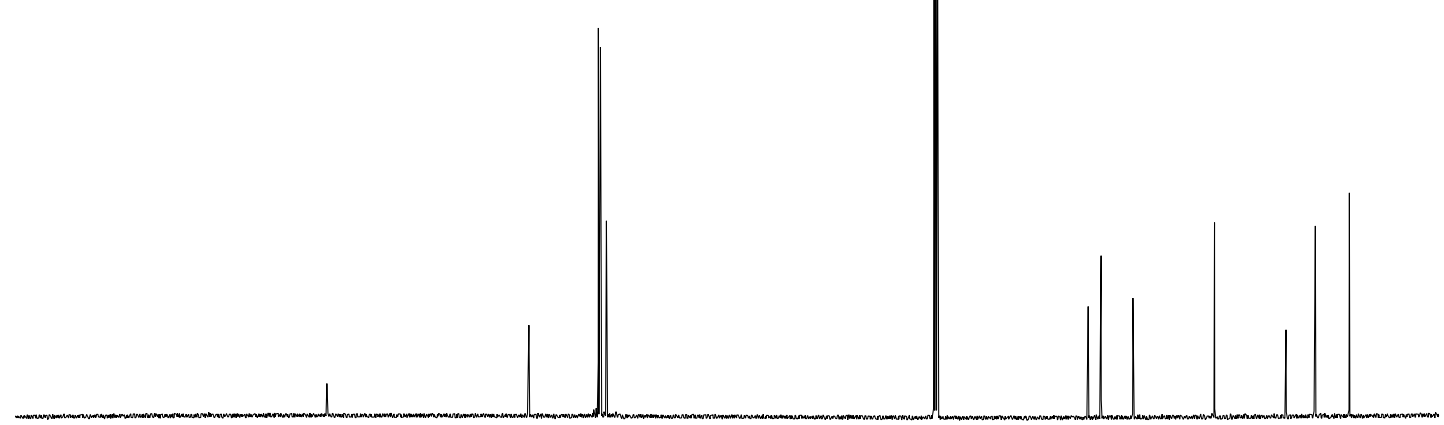




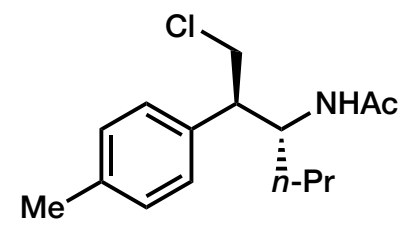

$N$-((2R*,3R*)-1-Chloro-2-(p-tolyl)hexan-3-yl)acetamide 6c

General Procedure D: Aziridine 5c (69 mg, $0.30 \mathrm{mmol})$ was employed. Purification of the residue by column chromatography (10-50\%, ethyl acetate in hexanes) afforded the title compound (40 $\mathrm{mg}, 50 \%)$ as a colorless oil.

${ }^{1} \mathrm{H}$ NMR $\left(500 \mathrm{MHz}, \mathrm{CDCl}_{3}\right) \delta 7.15(\mathrm{~d}, J=7.8 \mathrm{~Hz}, 2 \mathrm{H}), 7.08(\mathrm{~d}, J=8.1 \mathrm{~Hz}, 2 \mathrm{H}), 5.37(\mathrm{~d}, J=9.6$ $\mathrm{Hz}, 1 \mathrm{H}), 4.32-4.25(\mathrm{~m}, 1 \mathrm{H}), 3.86(\mathrm{dd}, J=11.1,5.4 \mathrm{~Hz}, 1 \mathrm{H}), 3.71(\mathrm{dd}, J=11.1,8.9 \mathrm{~Hz}, 1 \mathrm{H}), 2.93$ (app td, $J=8.9,5.5 \mathrm{~Hz}, 1 \mathrm{H}), 2.34(\mathrm{~s}, 3 \mathrm{H}), 2.03(\mathrm{~s}, 3 \mathrm{H}), 1.38-1.29(\mathrm{~m}, 2 \mathrm{H}), 1.23-1.15(\mathrm{~m}, 1 \mathrm{H})$, $1.13-1.06(\mathrm{~m}, 1 \mathrm{H}), 0.80(\mathrm{t}, J=7.1 \mathrm{~Hz}, 3 \mathrm{H})$;

${ }^{13} \mathrm{C}$ NMR $\left(126 \mathrm{MHz} \mathrm{CDCl}_{3}\right) \delta 170.0,137.0,136.2,129.4,128.2,53.3,51.6,46.9,34.2,23.4,21.0$, 18.9, 13.7;

IR ( $\mathrm{NaCl}$, thin film) 3266, 2958, 1644, 1552, $1374 \mathrm{~cm}^{-1}$;

HRMS (ESI-TOF) $\mathrm{m} / \mathrm{z}$ calcd for $\mathrm{C}_{15} \mathrm{H}_{22} \mathrm{ONCINa}(\mathrm{M}+\mathrm{Na})^{+}:$290.1282, found 290.1277. 

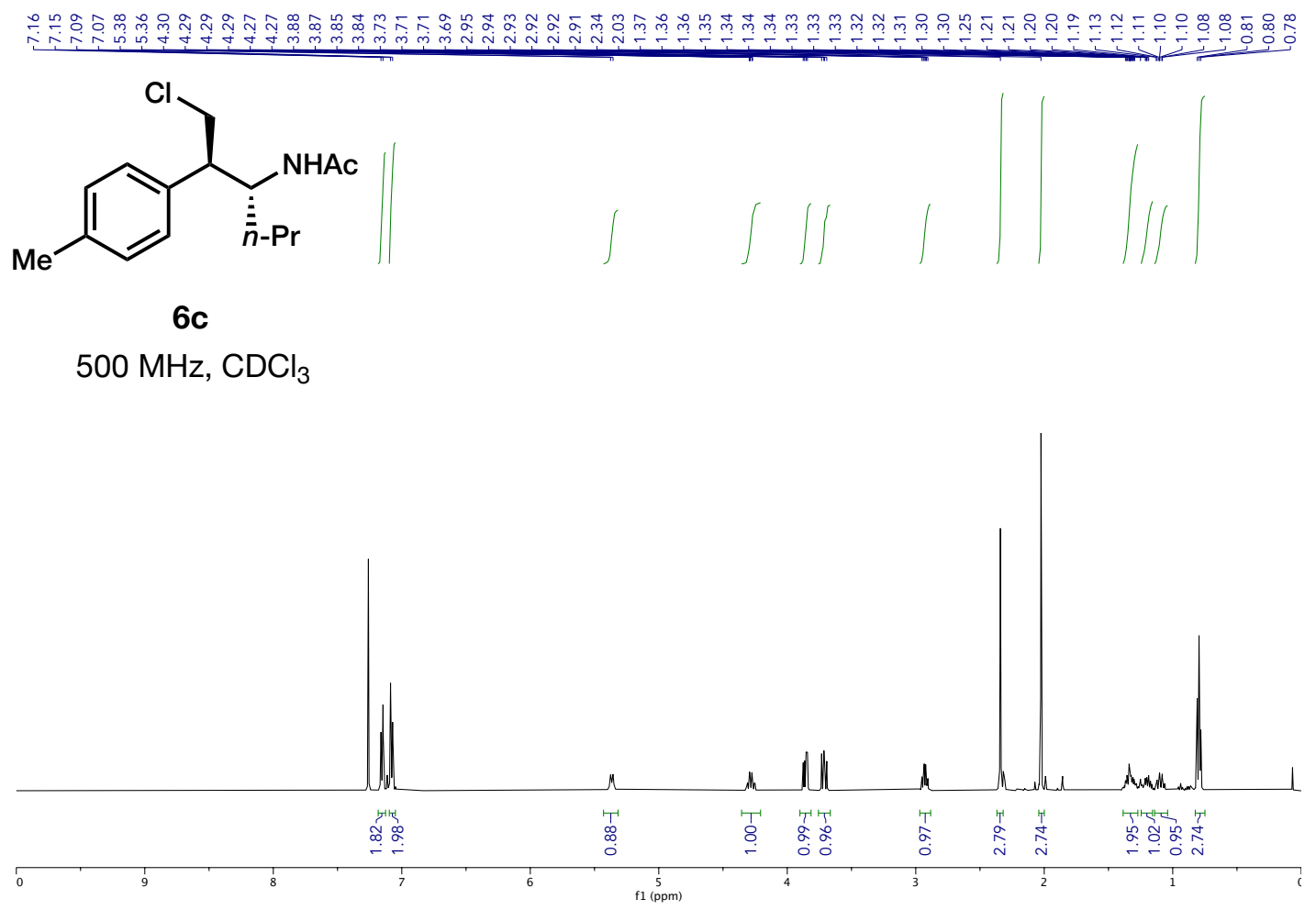<smiles>CCCC(N)[C@H](CCl)c1ccc(C)cc1</smiles>

$6 c$

$126 \mathrm{MHz}, \mathrm{CDCl}_{3}$

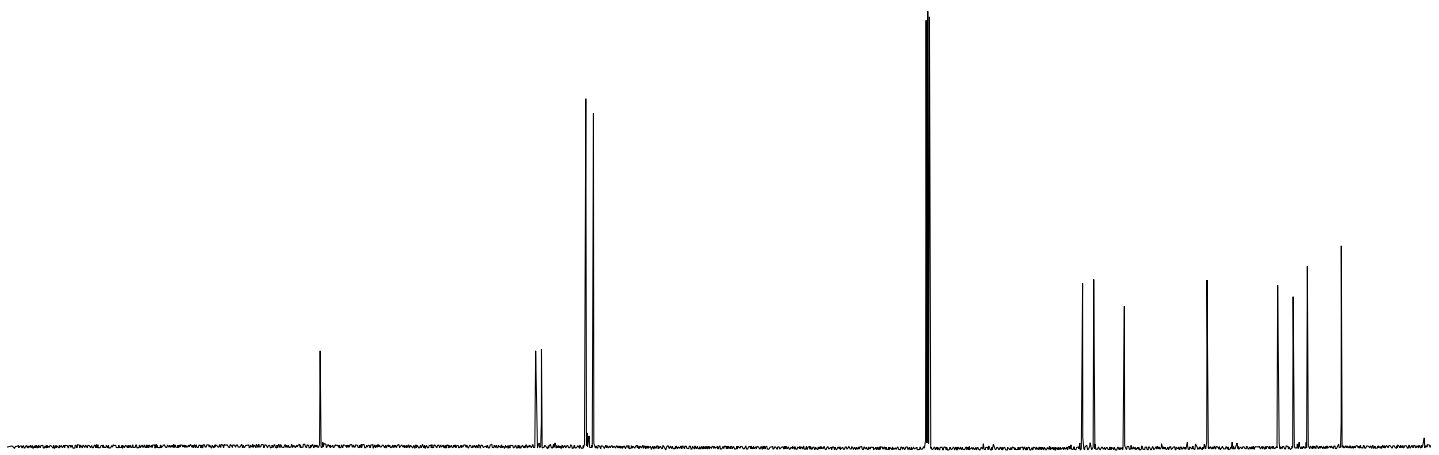

200

$180+160$

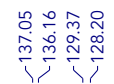

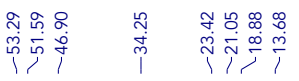

$120 \quad \mathrm{f1}(\mathrm{ppm})$

$100+\frac{1}{1}+1$ 20 


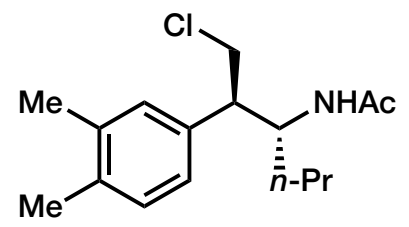

$N-\left(\left(2 R^{*}, 3 R^{*}\right)-1-C h l o r o-2-(3,4-d i m e t h y l p h e n y l) h e x a n-3-y l\right) a c e t a m i d e ~ 6 d$

General Procedure D: Aziridine 5d (74 mg, $0.30 \mathrm{mmol})$ was employed. Purification of the residue by column chromatography (10-45\%, ethyl acetate in hexanes) afforded the title compound (49 $\mathrm{mg}, 58 \%)$ as an orange oil.

${ }^{1} \mathrm{H}$ NMR $\left(500 \mathrm{MHz}, \mathrm{CDCl}_{3}\right) \delta 7.10(\mathrm{~d}, J=7.6 \mathrm{~Hz}, 1 \mathrm{H}), 6.95-6.90(\mathrm{~m}, 2 \mathrm{H}), 5.43(\mathrm{~d}, J=6.8 \mathrm{~Hz}, 1 \mathrm{H})$, 4.28 (app qd, $J=9.8,2.6 \mathrm{~Hz}, 1 \mathrm{H}$ ), 3.85 (dd, $J=11.0,5.5 \mathrm{~Hz}, 1 \mathrm{H}), 3.71$ (dd, $J=11.1,8.8 \mathrm{~Hz}, 1 \mathrm{H}$ ), 2.89 (app td, $J=8.8,5.4 \mathrm{~Hz}, 1 \mathrm{H}), 2.26(\mathrm{~s}, 3 \mathrm{H}), 2.24(\mathrm{~s}, 3 \mathrm{H}), 2.04(\mathrm{~s}, 3 \mathrm{H}), 1.40-1.28(\mathrm{~m}, 2 \mathrm{H}), 1.23-$ $1.17(\mathrm{~m}, 1 \mathrm{H}), 1.13-1.06(\mathrm{~m}, 1 \mathrm{H}), 0.80(\mathrm{t}, J=7.1 \mathrm{~Hz}, 3 \mathrm{H})$;

${ }^{13} \mathrm{C}$ NMR $\left(126 \mathrm{MHz}, \mathrm{CDCl}_{3}\right) \delta 170.1,136.8,136.6,135.7,129.8,129.5,125.7,53.3,51.6,47.0$, $34.3,23.4,19.9,19.4,18.9,13.7$;

IR ( $\mathrm{NaCl}$, thin film) 3267, 2958, 1644, 1553, 1452, $1374 \mathrm{~cm}^{-1}$;

HRMS (ESI-TOF) $\mathrm{m} / \mathrm{z}$ calcd for $\mathrm{C}_{16} \mathrm{H}_{24} \mathrm{ONCINa}(\mathrm{M}+\mathrm{Na})^{+}: 304.1439$, found 304.1434. 

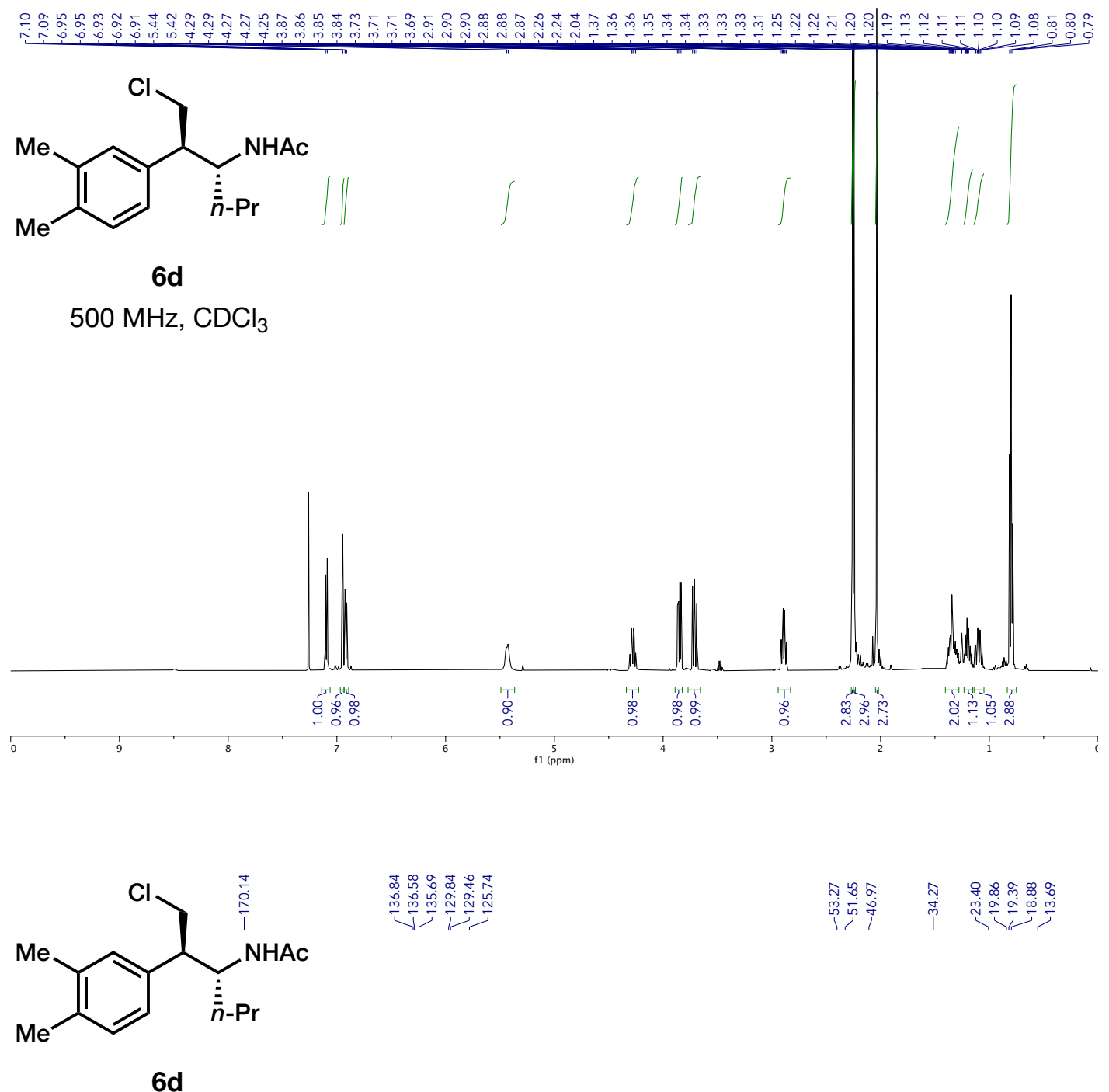

$126 \mathrm{MHz} \mathrm{CDCl}_{3}$

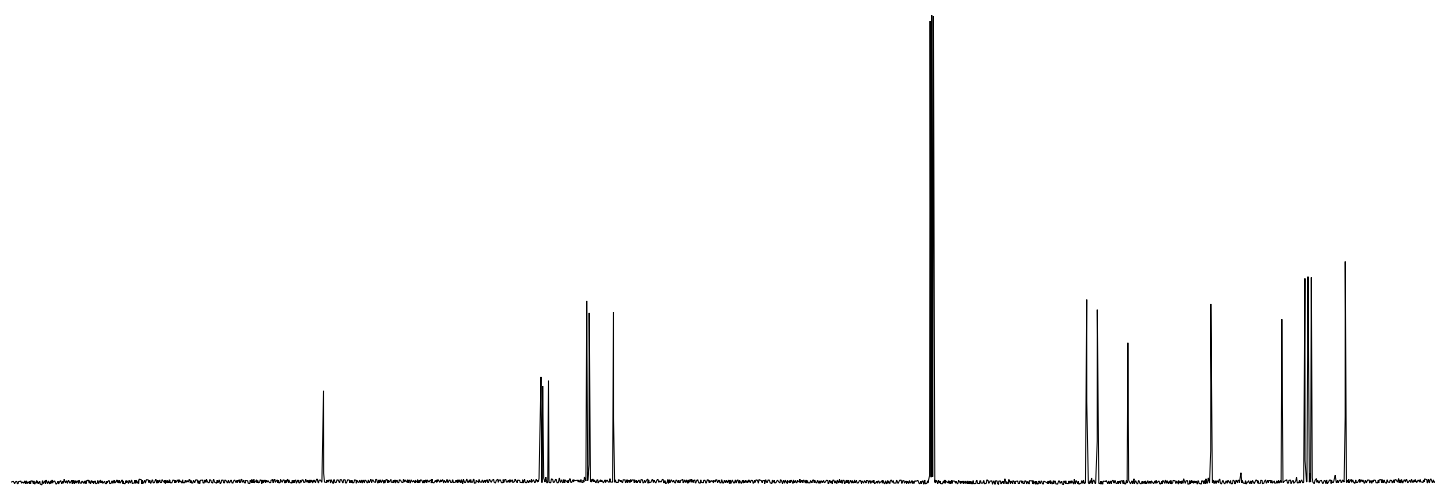

$200 \quad 180$ 160 140 $120 \quad \mathrm{f1}(\mathrm{ppm}) \quad 100$ 80 60 20 


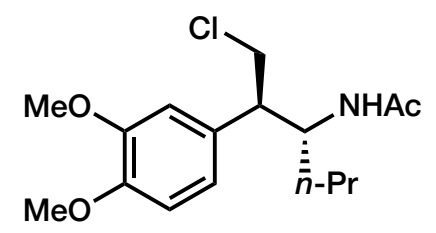

\section{$N-\left(\left(2 R^{*}, 3 R^{*}\right)-1-C h l o r o-2-(3,4-d i m e t h o x y p h e n y l) h e x a n-3-y l\right) a c e t a m i d e ~ 6 e$}

General Procedure D: Aziridine 5 e $(83 \mathrm{mg}, 0.30 \mathrm{mmol})$ was employed. Purification of the residue by column chromatography (10-55\%, ethyl acetate in hexanes) afforded the title compound (62 $\mathrm{mg}, 66 \%)$ as a yellow oil.

${ }^{1} \mathrm{H}$ NMR $\left(500 \mathrm{MHz}, \mathrm{CDCl}_{3}\right) \delta 6.84(\mathrm{~d}, J=8.2 \mathrm{~Hz}, 1 \mathrm{H}), 6.74(\mathrm{dd}, J=8.2,2.1 \mathrm{~Hz}, 1 \mathrm{H}), 6.69(\mathrm{~d}, J=$ $2.0 \mathrm{~Hz}, 1 \mathrm{H}), 5.29(\mathrm{~d}, J=9.6 \mathrm{~Hz}, 1 \mathrm{H}), 4.25(\operatorname{app~qd}, J=9.8,2.6 \mathrm{~Hz}, 1 \mathrm{H}), 3.88(\mathrm{~s}, 3 \mathrm{H}), 3.87(\mathrm{~s}, 3 \mathrm{H})$, 3.85 (dd, $J=11.1,5.2 \mathrm{~Hz}, 1 \mathrm{H}), 3.69$ (dd, $J=11.1,8.8 \mathrm{~Hz}, 1 \mathrm{H}), 2.87$ (app td, $J=9.0,5.3 \mathrm{~Hz}, 1 \mathrm{H})$, $2.03(\mathrm{~s}, 3 \mathrm{H}), 1.38-1.28(\mathrm{~m}, 2 \mathrm{H}), 1.23-1.16(\mathrm{~m}, 1 \mathrm{H}), 1.14-1.08(\mathrm{~m}, 1 \mathrm{H}), 0.80(\mathrm{t}, J=7.1 \mathrm{~Hz}, 3 \mathrm{H})$;

${ }^{13} \mathrm{C}$ NMR $\left(126 \mathrm{MHz}, \mathrm{CDCl}_{3}\right) \delta 170.1,149.0,148.3,131.8,120.6,111.1,111.0,56.0,55.8,53.5$, $51.7,47.0,34.5,23.5,18.9,13.7$;

IR ( $\mathrm{NaCl}$, thin film) 3269, 2958, 1647, 1518, $1260 \mathrm{~cm}^{-1}$;

HRMS (ESI-TOF) $\mathrm{m} / \mathrm{z}$ calcd for $\mathrm{C}_{16} \mathrm{H}_{24} \mathrm{O}_{3} \mathrm{NCINa}(\mathrm{M}+\mathrm{Na})^{+}:$336.1337, found 336.1338. 

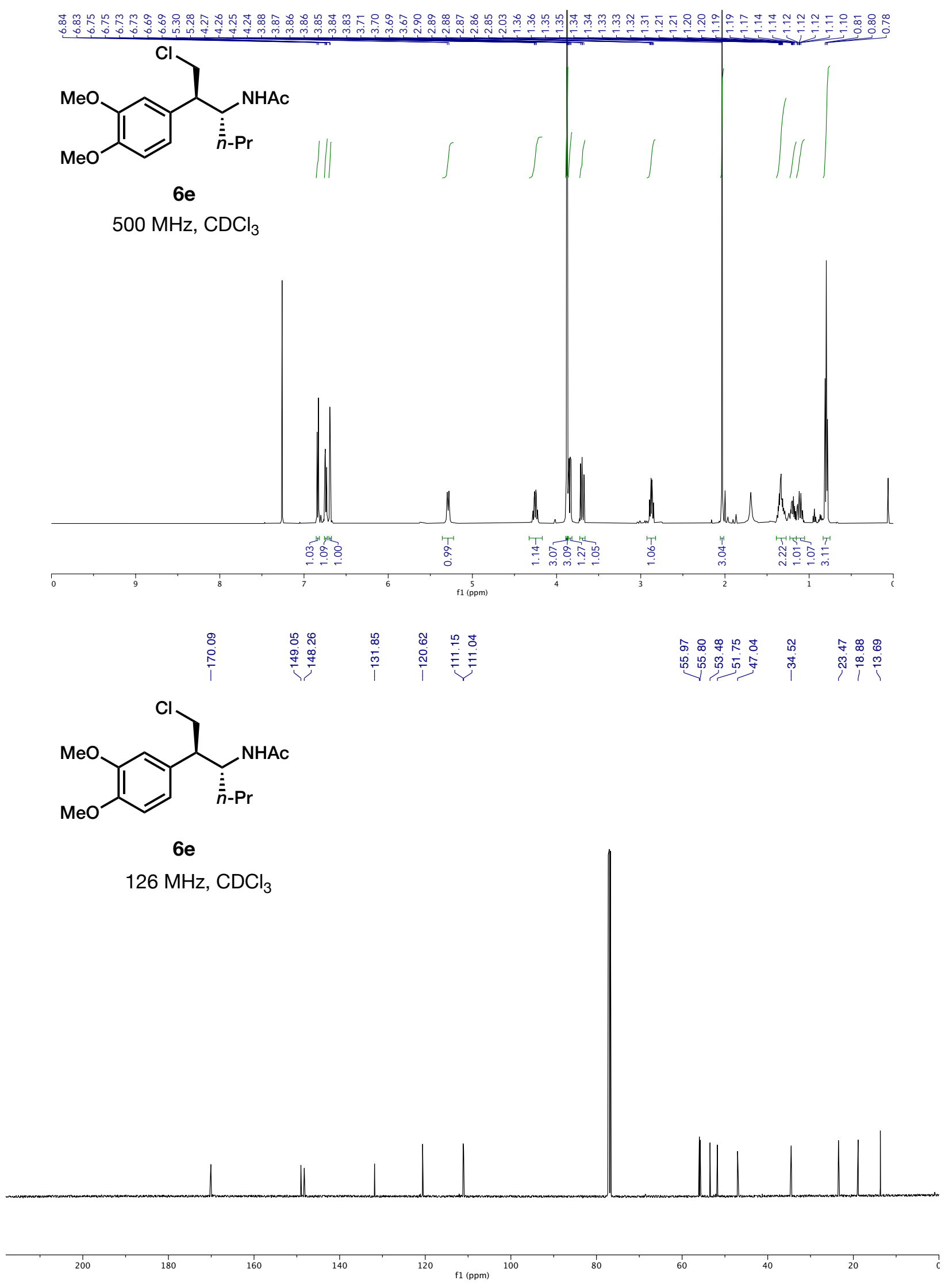
<smiles>CCCN[C@H](C(=O)O)[C@@H](CCl)c1ccc(OC)c(Br)c1</smiles>

\section{$N$-((2R*,3R*)-2-(3-Bromo-4-methoxyphenyl)-1-chlorohexan-3-yl)acetamide $6 f$}

General Procedure D: Aziridine $\mathbf{5 f}$ (98 $\mathrm{mg}, 0.30 \mathrm{mmol})$ was employed. Purification of the residue by column chromatography (5-45\%, ethyl acetate in hexanes) afforded the title compound (44 $\mathrm{mg}, 40 \%)$ as a yellow oil.

${ }^{1} \mathrm{H}$ NMR $\left(500 \mathrm{MHz}, \mathrm{CDCl}_{3}\right) \delta 7.38(\mathrm{~d}, J=2.2 \mathrm{~Hz}, 1 \mathrm{H}), 7.12(\mathrm{dd}, J=8.4,2.2 \mathrm{~Hz}, 1 \mathrm{H}), 6.88(\mathrm{~d}, J=$ $8.4 \mathrm{~Hz}, 1 \mathrm{H}), 5.25(\mathrm{~d}, J=9.5 \mathrm{~Hz}, 1 \mathrm{H}), 4.27-4.17(\mathrm{~m}, 1 \mathrm{H}), 3.90(\mathrm{~s}, 3 \mathrm{H}), 3.83(\mathrm{dd}, J=11.2,5.1 \mathrm{~Hz}$, $1 \mathrm{H}), 3.66(\mathrm{dd}, J=11.2,9.1 \mathrm{~Hz}, 1 \mathrm{H}), 2.88(\mathrm{td}, J=9.1,5.1 \mathrm{~Hz}, 1 \mathrm{H}), 2.04(\mathrm{~s}, 3 \mathrm{H}), 1.40-1.28(\mathrm{~m}, 2 \mathrm{H})$, 1.23-1.16 (m, 1H), 1.14-.07 (m, 1H), 0.81 (t, $J=7.0 \mathrm{~Hz}, 3 \mathrm{H})$;

${ }^{13} \mathrm{C}$ NMR $\left(126 \mathrm{MHz}, \mathrm{CDCl}_{3}\right) \delta 170.2,155.2,133.1,132.9,128.2,112.0,111.8,56.2,52.7,51.8$, 46.7, 34.4, 23.4, 18.9, 13.7;

IR ( $\mathrm{NaCl}$, thin film) 3241, 2873, 1641, 1499, $1284 \mathrm{~cm}^{-1}$;

HRMS $\left(\mathrm{Cl}^{+}\right) \mathrm{m} / \mathrm{z}$ calcd for $\mathrm{C}_{15} \mathrm{H}_{21} \mathrm{BrCINO}_{2} \mathrm{NH}_{4}\left(\mathrm{M}+\mathrm{NH}_{4}\right)^{+}:$364.0496, found 364.0495. 

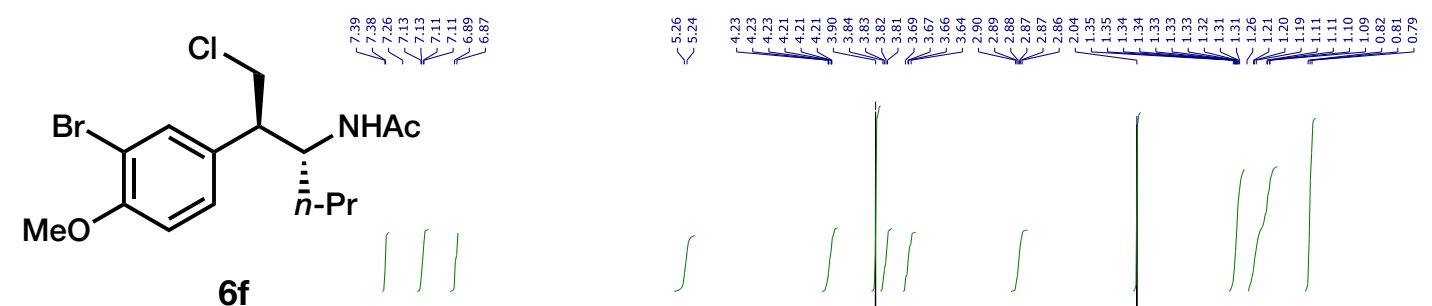

$500 \mathrm{MHz}, \mathrm{CDCl}_{3}$

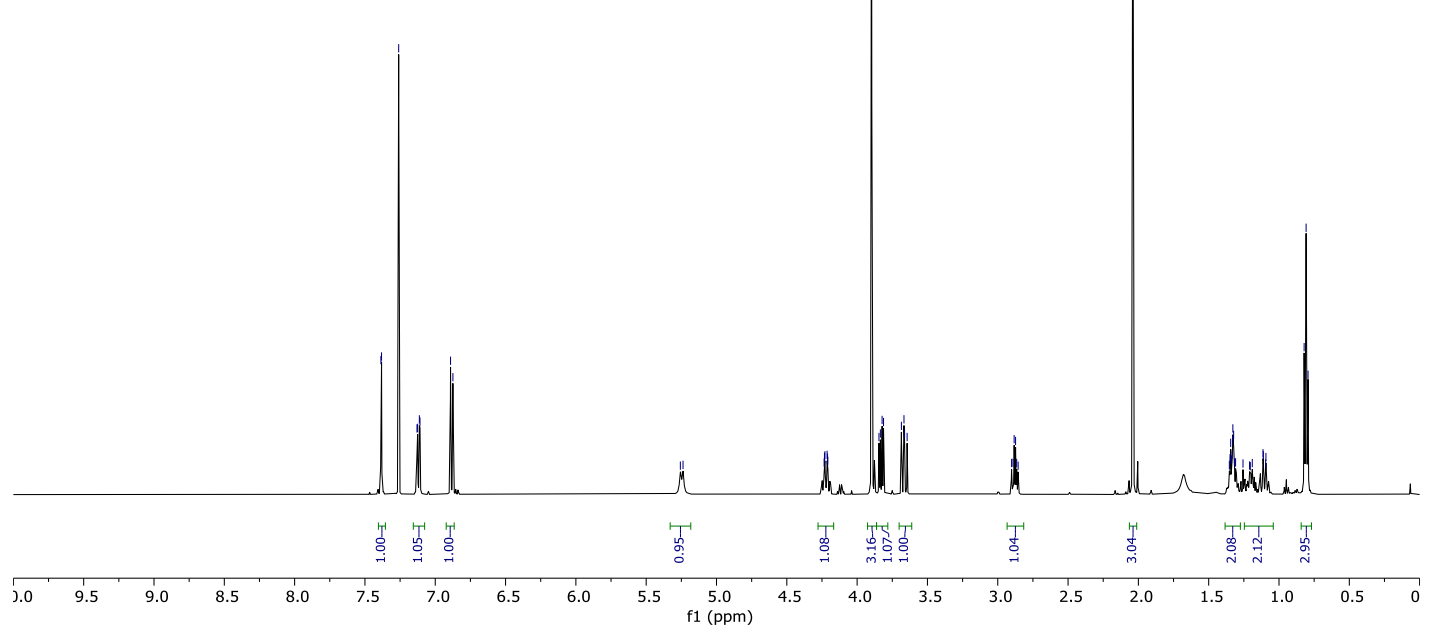

\begin{tabular}{|c|c|c|c|c|c|}
\hline 号 & 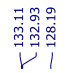 & 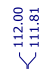 & 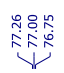 & 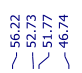 & $\stackrel{q}{f}$ \\
\hline
\end{tabular}

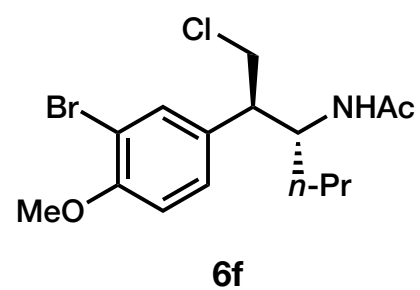

$126 \mathrm{MHz}, \mathrm{CDCl}_{3}$

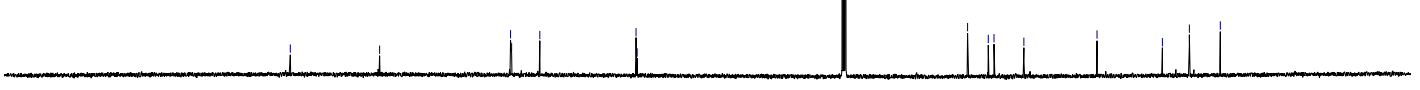

$\begin{array}{lllllllllllllllllllllllll}1 & 1 \\ 210 & 200 & 190 & 180 & 170 & 160 & 150 & 140 & 130 & 120 & 110 & 100 & 10 & 10 & 70 & 60 & 50 & 40 & 30 & 20 & 10 & 0 & -10 \\ \mathrm{f} 1(\mathrm{ppm})\end{array}$ 
<smiles>CCCC(N)[C@H](CCl)c1ccc(N2CCOCC2)cc1</smiles>

\section{$N-\left(\left(2 R^{*}, 3 R^{*}\right)-1-C h l o r o-2-(4-m o r p h o l i n o p h e n y l) h e x a n-3-y l\right) a c e t a m i d e ~ 6 g$}

General Procedure D: Aziridine $5 \mathrm{~g}$ (60 mg, $0.20 \mathrm{mmol})$ was employed. Purification of the residue by column chromatography (5-30\%, ethyl acetate in hexanes) afforded the title compound (39 $\mathrm{mg}, 58 \%)$ as a yellow oil.

${ }^{1} \mathrm{H}$ NMR $\left(500 \mathrm{MHz}, \mathrm{CDCl}_{3}\right) \delta$ 7.17-7.06 (m, 2H), $6.89(\mathrm{~d}, J=8.3 \mathrm{~Hz}, 2 \mathrm{H}), 5.18(\mathrm{~d}, J=9.7 \mathrm{~Hz}, 1 \mathrm{H})$, 4.32-4.22 (m, 1H), 3.90-3.76 (m, 5H), $3.69(\mathrm{dd}, J=11.1,8.7 \mathrm{~Hz}, 1 \mathrm{H}), 3.17(\mathrm{dd}, J=5.7,4.0 \mathrm{~Hz}$, $4 \mathrm{H}), 2.90(\mathrm{td}, J=8.7,5.6 \mathrm{~Hz}, 1 \mathrm{H}), 2.02(\mathrm{~s}, 3 \mathrm{H}), 1.60-1.01(\mathrm{~m}, 4 \mathrm{H}), 0.80(\mathrm{t}, J=7.2 \mathrm{~Hz}, 3 \mathrm{H})$;

${ }^{13} \mathrm{C}$ NMR $\left(126 \mathrm{MHz} \mathrm{CDCl}_{3}\right) \delta 167.0,150.3,130.3,129.1,115.5,66.9,52.8,51.7,49.0,46.9,34.2$, 23.5, 18.9, 13.7;

IR $\left(\mathrm{NaCl}\right.$, thin film) 3403, 2852, 1647, 1516, 1376, $1303 \mathrm{~cm}^{-1}$;

HRMS (ESI-TOF) $m / z$ calcd for $\mathrm{C}_{18} \mathrm{H}_{27} \mathrm{CIN}_{2} \mathrm{O}_{2} \mathrm{Na}(\mathrm{M}+\mathrm{Na})^{+}:$361.1653, found 361.1649. 

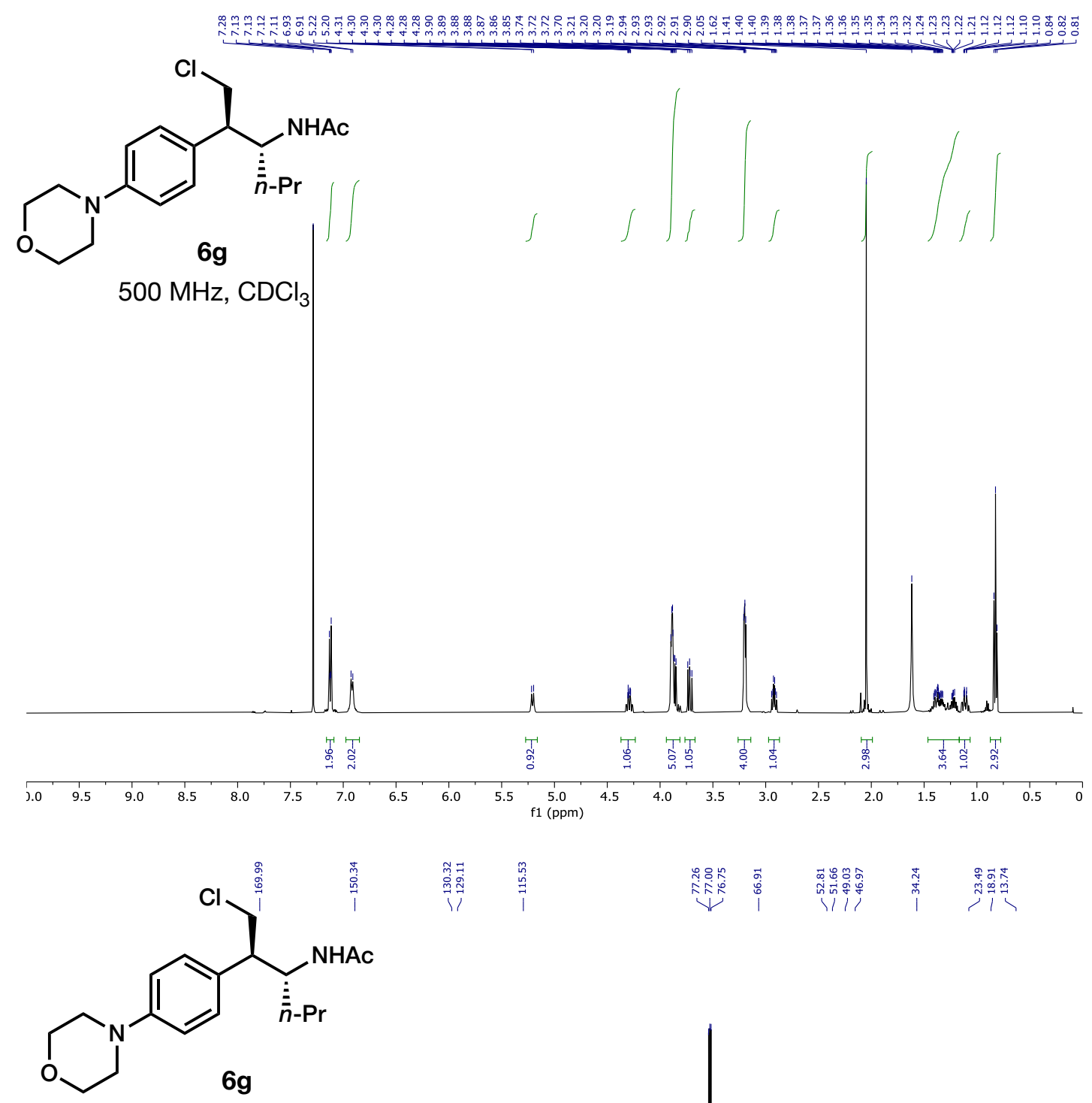

佔

$126 \mathrm{MHz}, \mathrm{CDCl}_{3}$
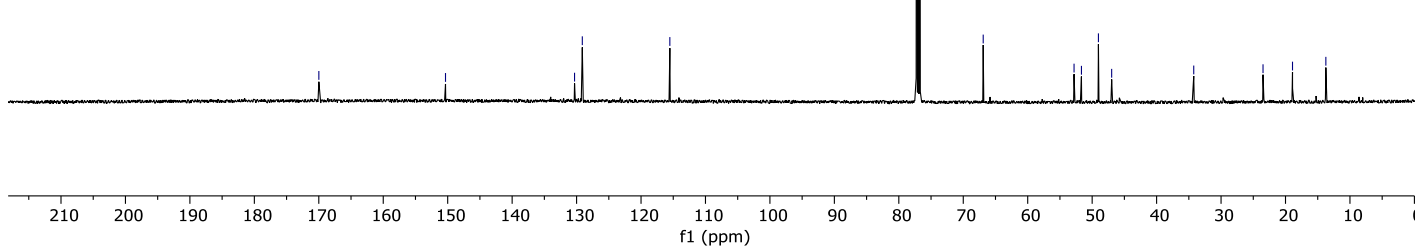


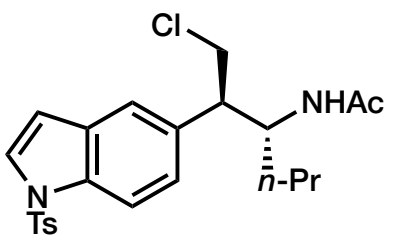

$N-\left(\left(2 R^{*}, 3 R^{*}\right)-1-C h l o r o-2-(1-t o s y l-1 H-i n d o l-5-y l) h e x a n-3-y l\right) a c e t a m i d e ~ 6 h$

General Procedure D: Aziridine 5h (123 mg, $0.30 \mathrm{mmol})$ was employed. Purification of the residue by column chromatography (5-40\%, ethyl acetate in hexanes) afforded the title compound (75 $\mathrm{mg}, 56 \%$ ) as a yellow solid.

${ }^{1} \mathrm{H}$ NMR $\left(500 \mathrm{MHz}, \mathrm{CDCl}_{3}\right) \delta 7.94(\mathrm{~d}, J=8.5 \mathrm{~Hz}, 1 \mathrm{H}), 7.79(\mathrm{~d}, J=8.1 \mathrm{~Hz}, 2 \mathrm{H}), 7.56(\mathrm{~d}, J=3.7$ $\mathrm{Hz}, 1 \mathrm{H}), 7.36(\mathrm{~d}, J=1.7 \mathrm{~Hz}, 1 \mathrm{H}), 7.24(\mathrm{~d}, J=8.2 \mathrm{~Hz}, 2 \mathrm{H}), 7.14(\mathrm{dd}, J=8.5,1.7 \mathrm{~Hz}, 1 \mathrm{H}), 6.62(\mathrm{~d}$, $J=3.7 \mathrm{~Hz}, 1 \mathrm{H}$ ), $5.36(\mathrm{~d}, J=10.9 \mathrm{~Hz}, 1 \mathrm{H}$ ), 4.30 (qd, $J=9.8,2.3 \mathrm{~Hz}, 1 \mathrm{H}$ ), 3.88 (dd, $J=11.1,5.2$ $\mathrm{Hz}, 1 \mathrm{H}), 3.72(\mathrm{dd}, J=11.1,8.9 \mathrm{~Hz}, 1 \mathrm{H}), 3.01(\mathrm{td}, J=8.9,5.2 \mathrm{~Hz}, 1 \mathrm{H}), 2.35(\mathrm{~s}, 3 \mathrm{H}), 2.02(\mathrm{~s}, 3 \mathrm{H})$, 1.28 (ddd, $J=12.2,8.7,3.1 \mathrm{~Hz}, 2 \mathrm{H}), 1.21-1.02(\mathrm{~m}, 2 \mathrm{H}), 0.75$ (t, $J=7.0 \mathrm{~Hz}, 3 \mathrm{H}$ );

${ }^{13} \mathrm{C}$ NMR $\left(126 \mathrm{MHz}, \mathrm{CDCl}_{3}\right) \delta 170.1,145.1,135.3,134.4,134.0,131.0,129.9,126.9,126.7$, 124.8, 121.0, 113.6, 108.8, 53.7, 51.9, 47.1, 34.4, 23.4, 21.6, 18.9, 13.6;

IR ( $\mathrm{NaCl}$, thin film) 3255, 2959, 1648, 1534, 1460, $1371 \mathrm{~cm}^{-1}$;

HRMS (ESI-TOF) $\mathrm{m} / \mathrm{z}$ calcd for $\mathrm{C}_{23} \mathrm{H}_{27} \mathrm{CIN}_{2} \mathrm{O}_{3} \mathrm{SNa}(\mathrm{M}+\mathrm{Na})^{+}: 469.1323$, found 469.1338;

m. pt $97-101^{\circ} \mathrm{C}$ 

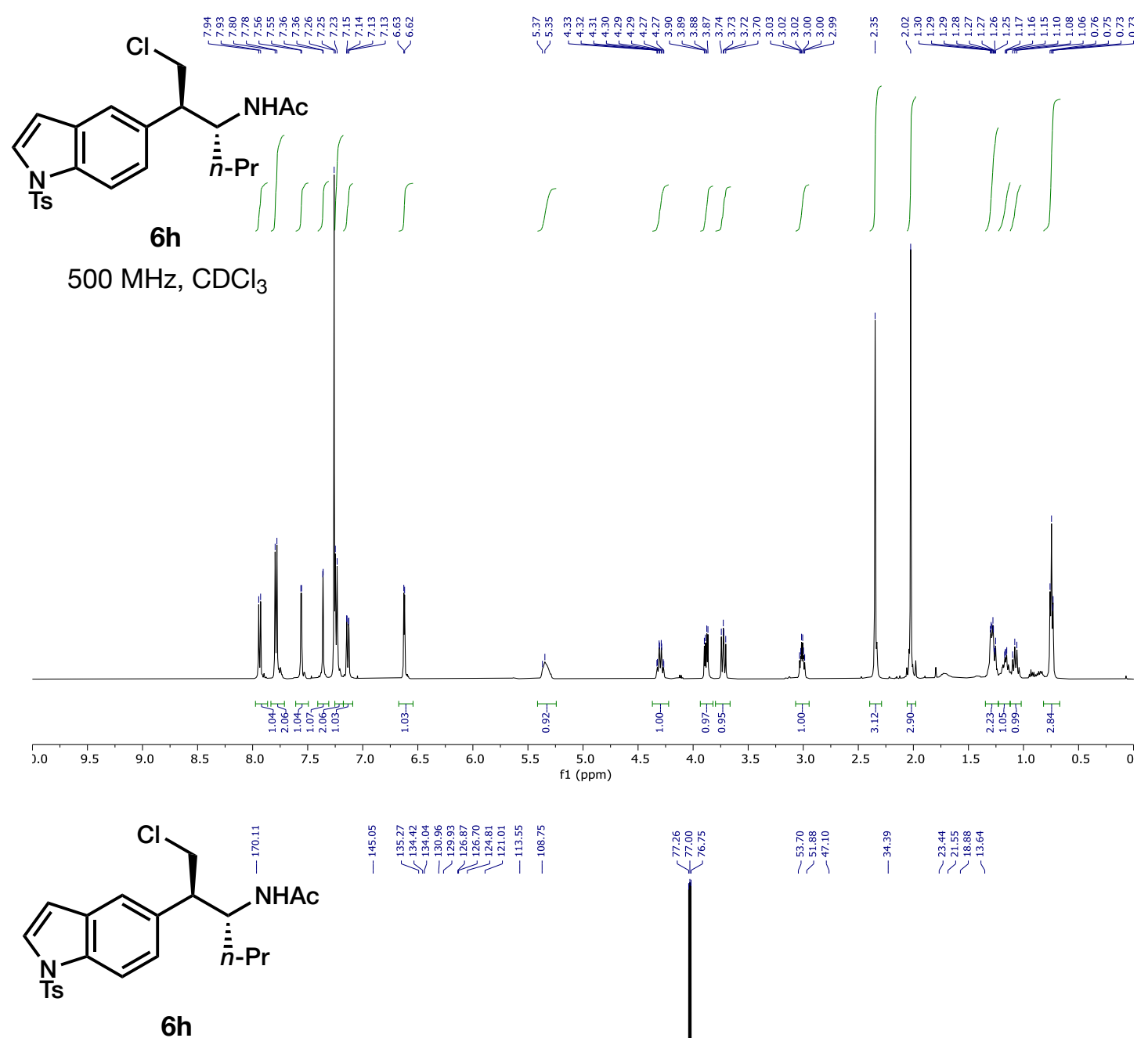

$126 \mathrm{MHz}, \mathrm{CDCl}_{3}$

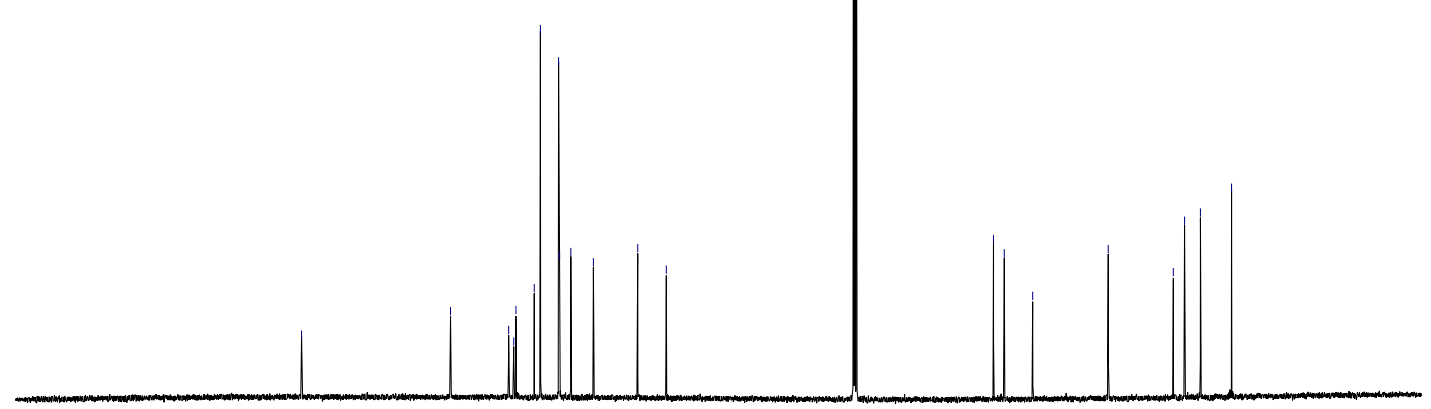

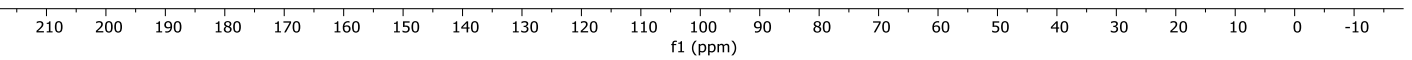


<smiles>CC[C@@H](N)[C@H](CCl)c1ccc2c(c1)CCO2</smiles>

$N-\left(\left(2 R^{*}, 3 R^{*}\right)-1-C h l o r o-2-(2,3-d i h y d r o b e n z o f u r a n-5-y l) h e x a n-3-y l\right) a c e t a m i d e ~ 6 i$

General Procedure D: Aziridine 5i (52 mg, $0.20 \mathrm{mmol})$ was employed. Purification of the residue by column chromatography (0-20\%, ethyl acetate in hexanes) afforded the title compound (37 $\mathrm{mg}, 60 \%)$ as a clear oil.

${ }^{1} \mathrm{H}$ NMR $\left(500 \mathrm{MHz}, \mathrm{CDCl}_{3}\right) \delta 7.03(\mathrm{~d}, J=1.9 \mathrm{~Hz}, 1 \mathrm{H}), 6.90(\mathrm{dd}, J=8.1,2.0 \mathrm{~Hz}, 1 \mathrm{H}), 6.74(\mathrm{~d}, J=$ $8.1 \mathrm{~Hz}, 1 \mathrm{H}), 5.35(\mathrm{~s}, 1 \mathrm{H}), 4.57(\mathrm{t}, J=8.7 \mathrm{~Hz}, 2 \mathrm{H}), 4.23(\mathrm{qd}, J=9.8,2.6 \mathrm{~Hz}, 1 \mathrm{H}), 3.84(\mathrm{dd}, J=$ 11.1, $5.4 \mathrm{~Hz}, 1 \mathrm{H}), 3.67$ (dd, $J=11.1,8.9 \mathrm{~Hz}, 1 \mathrm{H}), 3.21(\mathrm{t}, J=8.7 \mathrm{~Hz}, 2 \mathrm{H}), 2.87(\mathrm{dt}, J=8.9,4.4$ $\mathrm{Hz}, 1 \mathrm{H}), 2.03(\mathrm{~s}, 3 \mathrm{H}), 1.39-1.28(\mathrm{~d}, J=4.5 \mathrm{~Hz}, 2 \mathrm{H}), 1.27-1.14(\mathrm{~m}, 1 \mathrm{H}), 1.13-1.01(\mathrm{~m}, 1 \mathrm{H}), 0.80(\mathrm{t}$, $J=7.1 \mathrm{~Hz}, 3 \mathrm{H})$;

${ }^{13} \mathrm{C}$ NMR $\left(126 \mathrm{MHz}, \mathrm{CDCl}_{3}\right) \delta 170.1,159.4,131.2,128.2,127.5,124.4,109.2,71.3,53.2,51.9$, 47.2, 34.4, 29.7, 23.4, 18.9, 13.7;

IR (NaCl, thin film) 3255, 2959, 2934, 1644, 1493, $1242 \mathrm{~cm}^{-1}$;

HRMS (ESI-TOF) $\mathrm{m} / \mathrm{z}$ calcd for $\mathrm{C}_{16} \mathrm{H}_{22} \mathrm{CINO}_{2} \mathrm{Na}(\mathrm{M}+\mathrm{Na})^{+}: 318.1231$, found 318.1245. 


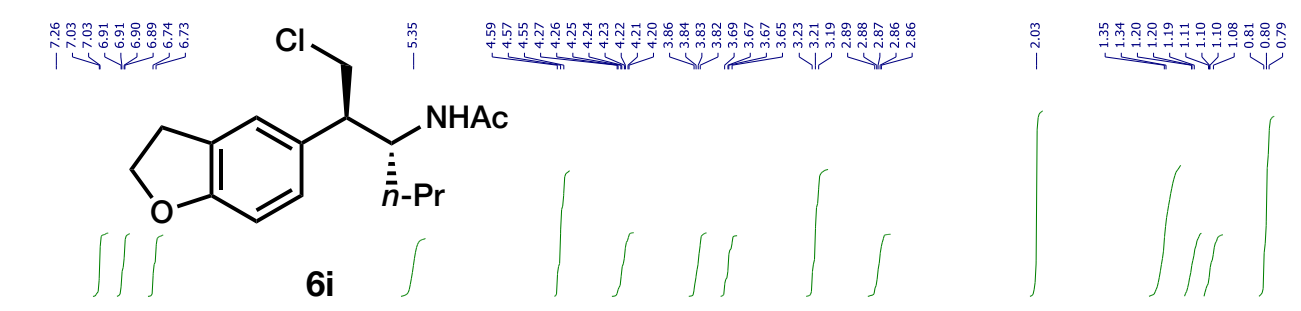

$500 \mathrm{MHz}, \mathrm{CDCl}_{3}$
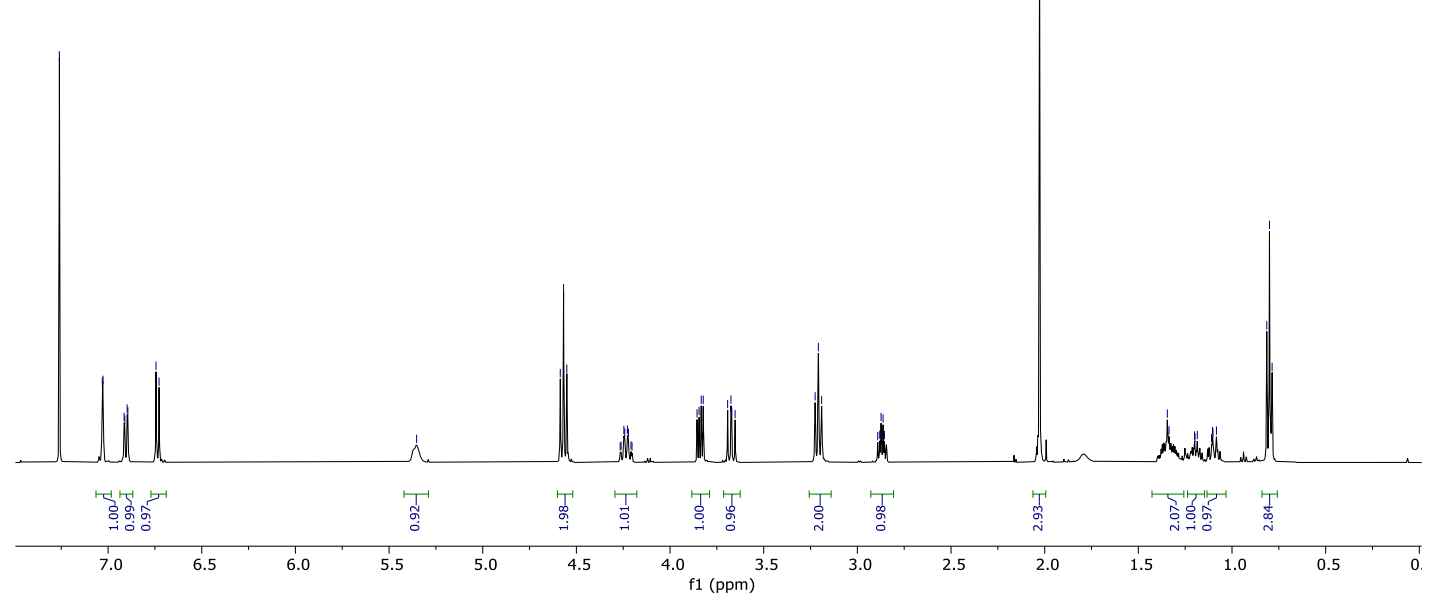<smiles>CCCC(N)[C@H](CCl)c1ccc2c(c1)CCO2</smiles>

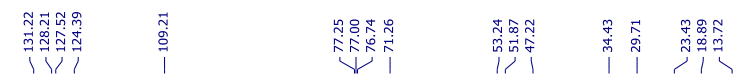

$6 i$

$126 \mathrm{MHz} \mathrm{CDCl}_{3}$

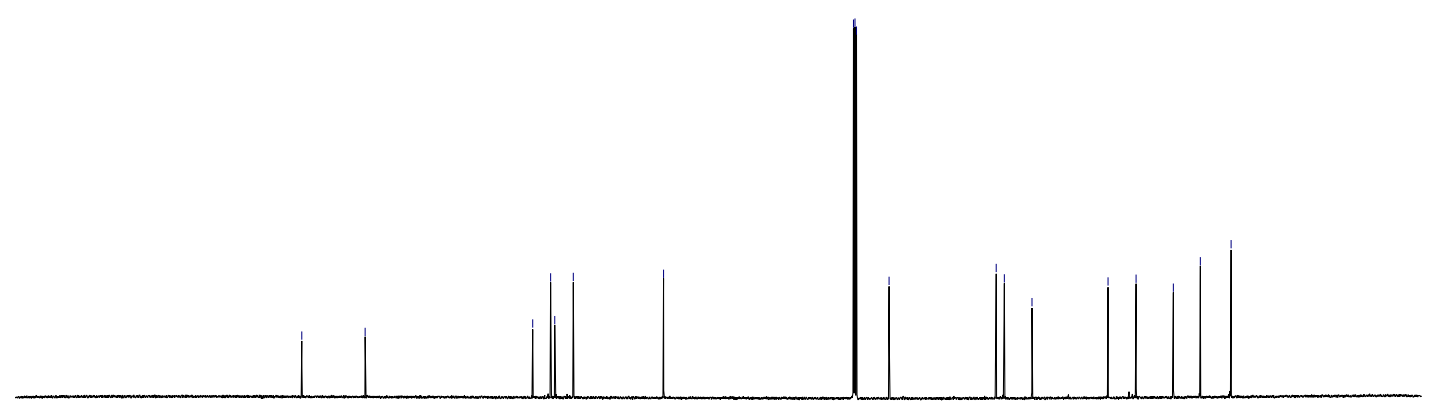

$\begin{array}{rllllllllllllllllllllll}1 & 1 \\ 210 & 200 & 190 & 180 & 170 & 160 & 150 & 140 & 130 & 120 & 110 & 100 & 90 & 80 & 70 & 60 & 50 & 40 & 30 & 20 & 10 & 0 & -10\end{array}$ 
<smiles>CN[C@H]([Po])[C@H](CCl)c1ccccc1OC</smiles>

\section{$N-\left(\left(2 R^{*}, 3 R^{*}\right)-1-C h l o r o-2-(2-m e t h o x y p h e n y l) h e x a n-3-y l\right) a c e t a m i d e ~ 6 j$}

General Procedure D: Aziridine 5j (74 mg, $0.30 \mathrm{mmol})$ was employed. Purification of the residue by column chromatography (10-45\%, ethyl acetate in hexanes) afforded the title compound (56 $\mathrm{mg}, 66 \%)$ as an orange solid.

${ }^{1} \mathrm{H}$ NMR $\left(500 \mathrm{MHz}, \mathrm{CDCl}_{3}\right) \delta$ 7.27-7.23 (m, 1H), $7.16(\mathrm{dd}, J=7.5,1.7 \mathrm{~Hz}, 1 \mathrm{H}), 6.94(\mathrm{td}, J=7.5$, $1.1 \mathrm{~Hz}, 1 \mathrm{H}), 6.89(\mathrm{dd}, J=8.4,1.1 \mathrm{~Hz}, 2 \mathrm{H}), 5.51(\mathrm{~d}, J=9.8 \mathrm{~Hz}, 1 \mathrm{H}), 3.87-3.82(\mathrm{~m}, 2 \mathrm{H}), 3.82(\mathrm{~s}$, $3 \mathrm{H}), 3.40-3.35(\mathrm{~m}, 1 \mathrm{H}), 2.02(\mathrm{~s}, 3 \mathrm{H}), 1.34-1.25(\mathrm{~m}, 2 \mathrm{H}), 1.23-1.17(\mathrm{~m}, 1 \mathrm{H}), 1.17-1.09(\mathrm{~m}, 1 \mathrm{H}), 0.77$ (t, $J=7.1 \mathrm{~Hz}, 3 \mathrm{H})$;

${ }^{13} \mathrm{C} \mathrm{NMR}\left(126 \mathrm{MHz}, \mathrm{CDCl}_{3}\right) \delta 170.1,157.5,129.2,128.3,127.7,120.7,110.9,55.4,51.0,47.9$, 46.3, 34.9, 23.5, 18.7, 13.7;

IR ( $\mathrm{NaCl}$, thin film) 3272, 2958, 1645, 1555, 1494, $1243 \mathrm{~cm}^{-1}$;

HRMS (ESI-TOF) $m / z$ calcd for $\mathrm{C}_{15} \mathrm{H}_{22} \mathrm{O}_{2} \mathrm{NCINa}(\mathrm{M}+\mathrm{Na})^{+}:$306.1231, found 306.1250;

m. pt $104-109^{\circ} \mathrm{C}$ 


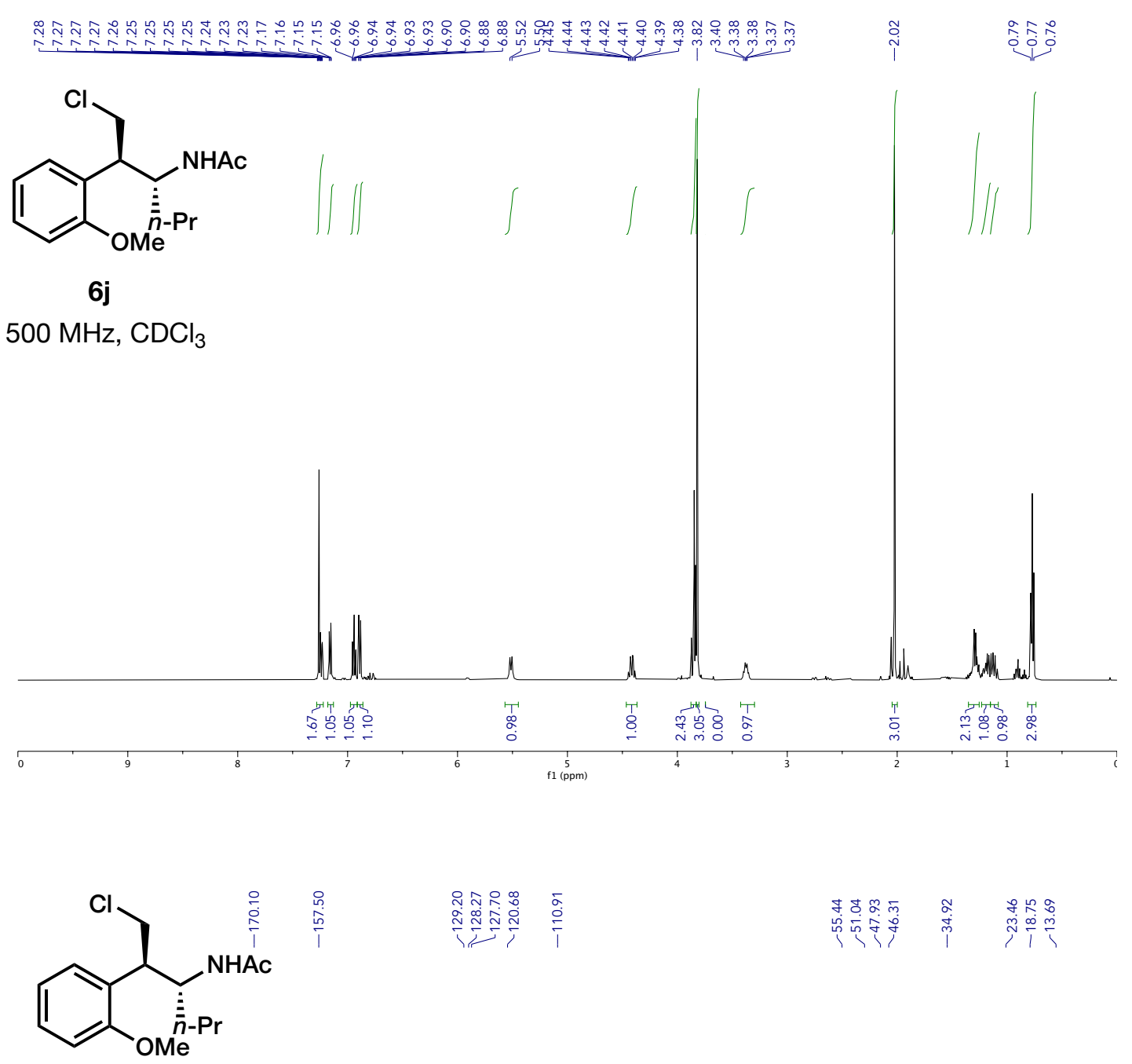

6j

$126 \mathrm{MHz}, \mathrm{CDCl}_{3}$

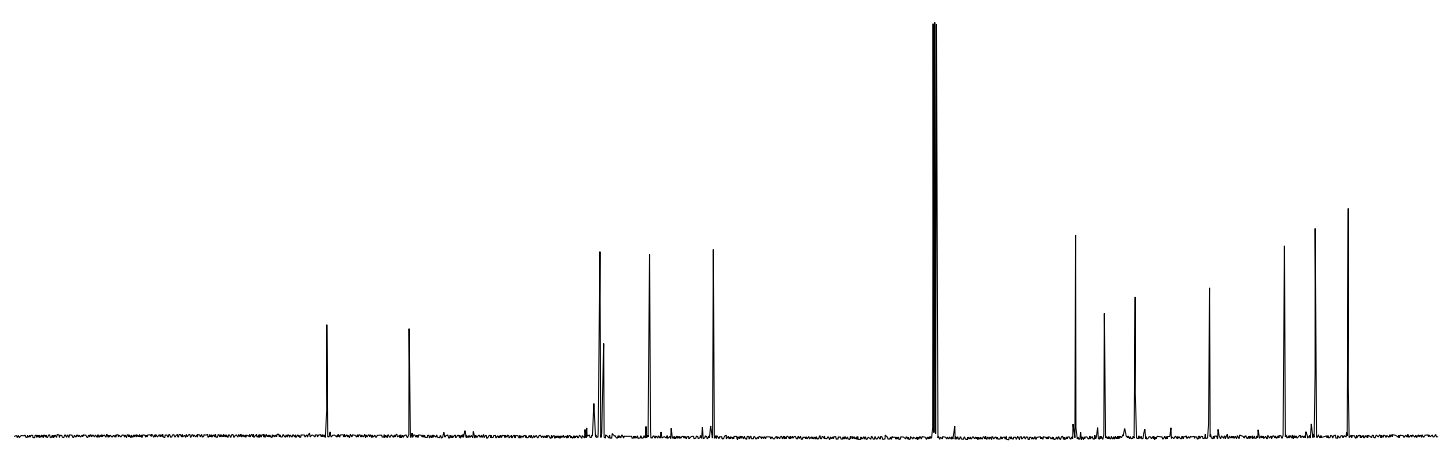


<smiles>COc1ccc([C@H](CCl)[C@@H](CC(C)C)NC(C)C)cc1</smiles>

\section{$N-\left(\left(2 R^{*}, 3 R^{*}\right)-1-C h l o r o-2-(4-m e t h o x y p h e n y l)-5-m e t h y l h e x a n-3-y l\right)$ acetamide $6 \mathrm{k}$}

General Procedure D: Aziridine 5k (52 mg, $0.20 \mathrm{mmol})$ was employed. Purification of the residue by column chromatography (5-20\%, ethyl acetate in hexanes) afforded the title compound (47 $\mathrm{mg}, 79 \%)$ as a clear oil.

${ }^{1} \mathrm{H}$ NMR $\left(500 \mathrm{MHz}, \mathrm{CDCl}_{3}\right) \delta 7.10(\mathrm{~d}, J=8.7 \mathrm{~Hz}, 2 \mathrm{H}), 6.88(\mathrm{~d}, J=8.7 \mathrm{~Hz}, 2 \mathrm{H}), 5.29(\mathrm{~s}, 1 \mathrm{H}), 4.39-$ $4.29(\mathrm{~m}, 1 \mathrm{H}), 3.87-3.82(\mathrm{dd}, J=11.0,5.6 \mathrm{~Hz}, 1 \mathrm{H}), 3.80(\mathrm{~s}, 3 \mathrm{H}), 3.73-3.65(\mathrm{~m}, 1 \mathrm{H}), 2.97-2.83(\mathrm{~m}$, $1 \mathrm{H}), 2.02(\mathrm{~s}, 3 \mathrm{H}), 1.56-1.48(\mathrm{~m}, 1 \mathrm{H}), 1.10$ (ddd, $J=8.1,4.4,2.2 \mathrm{~Hz}, 2 \mathrm{H}), 0.80(\mathrm{~d}, J=6.6 \mathrm{~Hz}, 6 \mathrm{H})$;

${ }^{13} \mathrm{C} \mathrm{NMR}\left(126 \mathrm{MHz} \mathrm{CDCl}_{3}\right) \delta 169.9,158.8,131.2,129.3,114.0,55.2,53.2,50.1,46.9,41.4,24.7$, 23.7, 23.4, 21.2;

IR ( $\mathrm{NaCl}$, thin film) 3385, 2956, 1643, 1548, 1466, $1366 \mathrm{~cm}^{-1}$;

HRMS (ESI-TOF) $\mathrm{m} / \mathrm{z}$ calcd for $\mathrm{C}_{16} \mathrm{H}_{24} \mathrm{CINO}_{2} \mathrm{H}(\mathrm{M}+\mathrm{H})^{+}: 320.1388$, found 320.1362. 


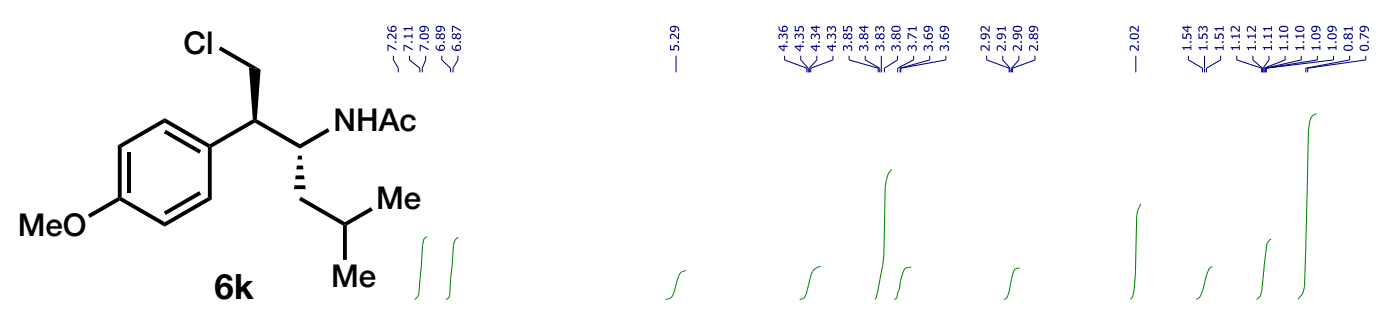

$500 \mathrm{MHz}, \mathrm{CDCl}_{3}$
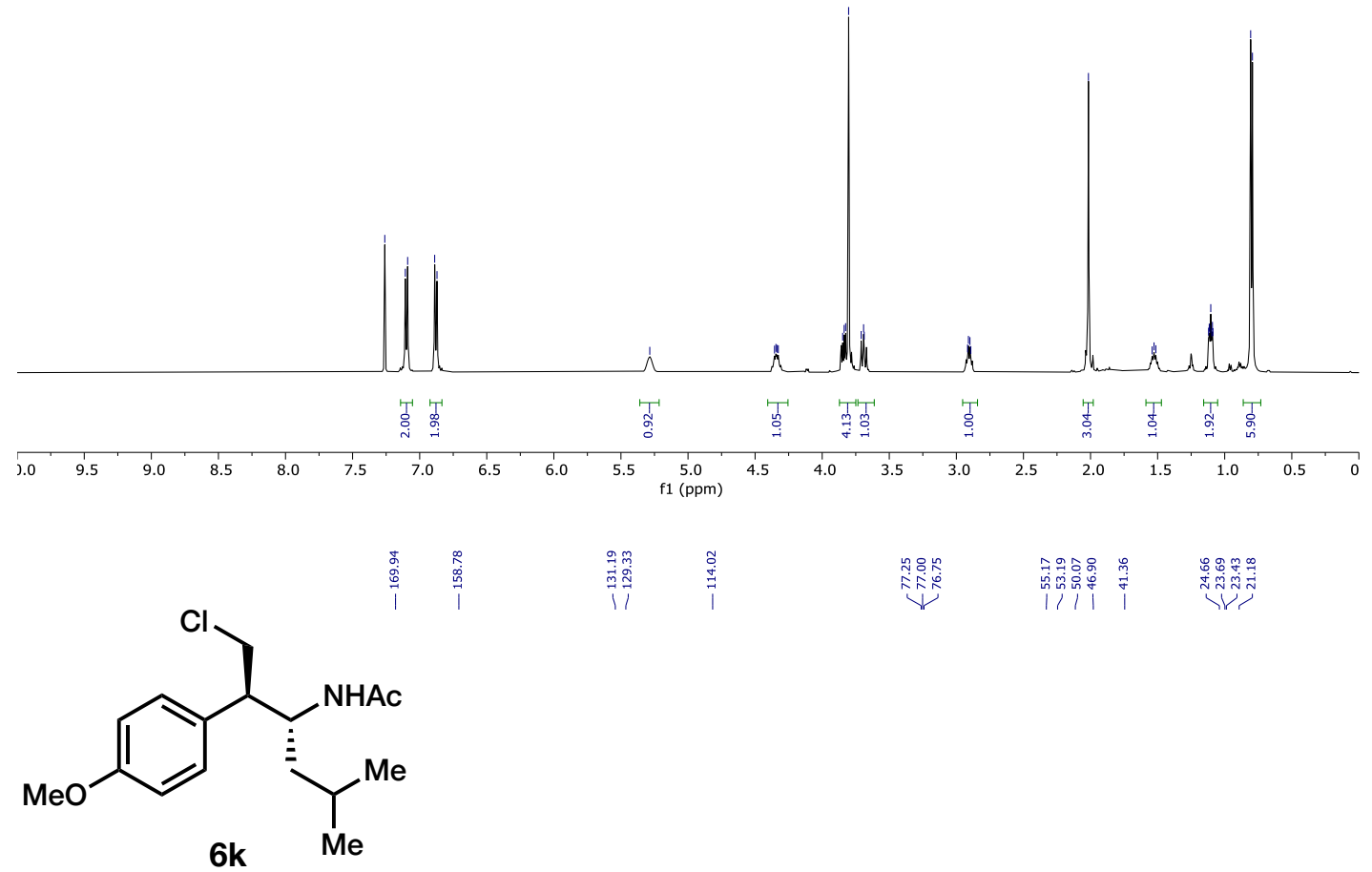

$126 \mathrm{MHz}, \mathrm{CDCl}_{3}$

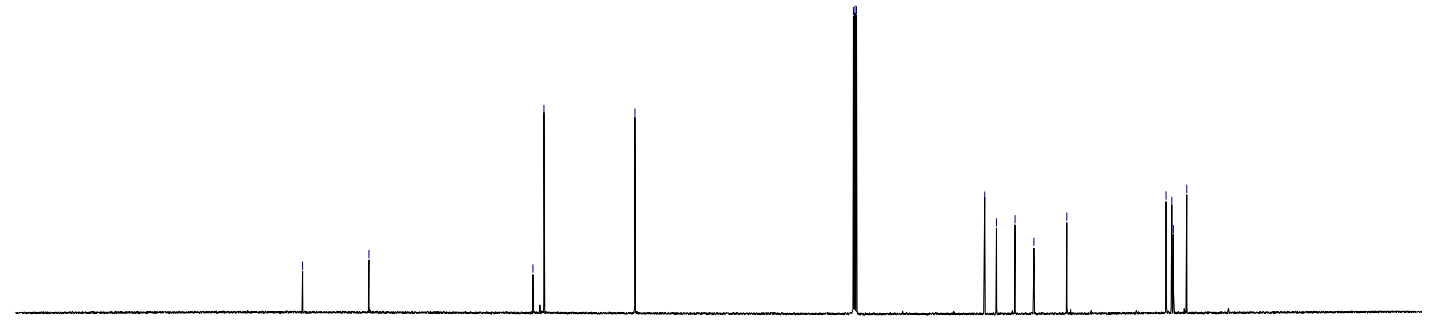

$\begin{array}{rllllllllllllllllllllll}1 & 1 \\ 210 & 200 & 190 & 180 & 170 & 160 & 150 & 140 & 130 & 120 & 110 & 100 & 90 & 80 & 70 & 60 & 50 & 40 & 30 & 20 & 10 & 0 & -10\end{array}$ 
<smiles>CN[C@H](C(C)C)[C@@H](CCl)c1ccc(OC)cc1</smiles>

\section{$N-\left(\left(2 R^{*}, 3 R^{*}\right)-1-C h l o r o-2-(4-m e t h o x y p h e n y l)-4-m e t h y l p e n t a n-3-y l\right) a c e t a m i d e ~ 6 I$}

General Procedure D: Aziridine $5 \mathrm{I}(72 \mathrm{mg}, 0.20 \mathrm{mmol})$ was employed. Purification of the residue by column chromatography (10-20\%, ethyl acetate in hexanes) afforded the title compound (33 $\mathrm{mg}, 42 \%)$ as a yellow oil.

${ }^{1} \mathrm{H}$ NMR $\left(500 \mathrm{MHz}, \mathrm{CDCl}_{3}\right) \delta 7.80(\mathrm{~d}, J=8.3 \mathrm{~Hz}, 2 \mathrm{H}), 7.31(\mathrm{~d}, J=7.7 \mathrm{~Hz}, 2 \mathrm{H}), 7.09(\mathrm{~d}, J=8.7$ $\mathrm{Hz}, 2 \mathrm{H}), 6.86(\mathrm{~d}, J=8.7 \mathrm{~Hz}, 2 \mathrm{H}), 4.68(\mathrm{~d}, J=9.8 \mathrm{~Hz}, 1 \mathrm{H}), 4.01(\mathrm{dd}, J=11.1,4.6 \mathrm{~Hz}, 1 \mathrm{H}), 3.79$ (s, 3H), 3.61-3.51 (m, 2H), $2.87(\mathrm{~d}, J=4.7 \mathrm{~Hz}, 1 \mathrm{H}), 2.42(\mathrm{~s}, 3 \mathrm{H}), 1.52(\mathrm{dd}, J=6.9,2.6 \mathrm{~Hz}, 1 \mathrm{H})$, $0.67(\mathrm{~d}, J=6.8 \mathrm{~Hz}, 3 \mathrm{H}), 0.47(\mathrm{~d}, J=6.9 \mathrm{~Hz}, 3 \mathrm{H})$;

${ }^{13} \mathrm{C}$ NMR $\left(126 \mathrm{MHz}, \mathrm{CDCl}_{3}\right) \delta 158.8,143.4,138.6,131.9,129.6,128.9,126.9,114.1,62.2,55.2$, 51.4, 48.2, 28.9, 21.5, 20.5, 14.8;

IR ( $\mathrm{NaCl}$, thin film) 3291, 2962, 1513, 1460, $1325 \mathrm{~cm}^{-1}$;

HRMS $\left(\mathrm{Cl}^{+}\right) \mathrm{m} / \mathrm{z}$ calcd for $\mathrm{C}_{20} \mathrm{H}_{26} \mathrm{CINO}_{3} \mathrm{SNH}_{4}\left(\mathrm{M}+\mathrm{NH}_{4}\right)^{+}:$413.1666, found 413.1656. 

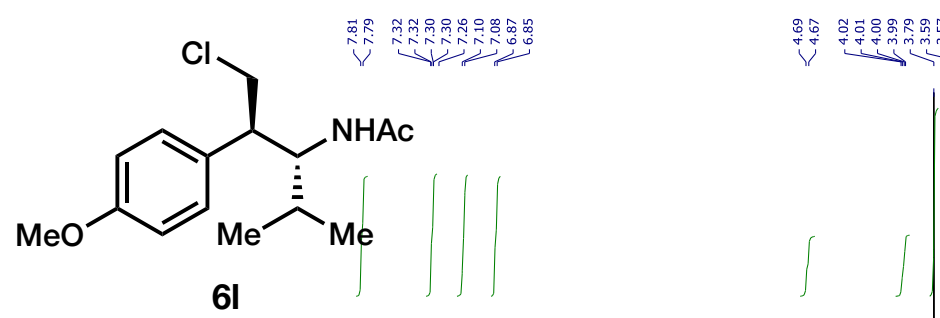

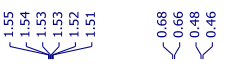

$500 \mathrm{MHz}, \mathrm{CDCl}_{3}$
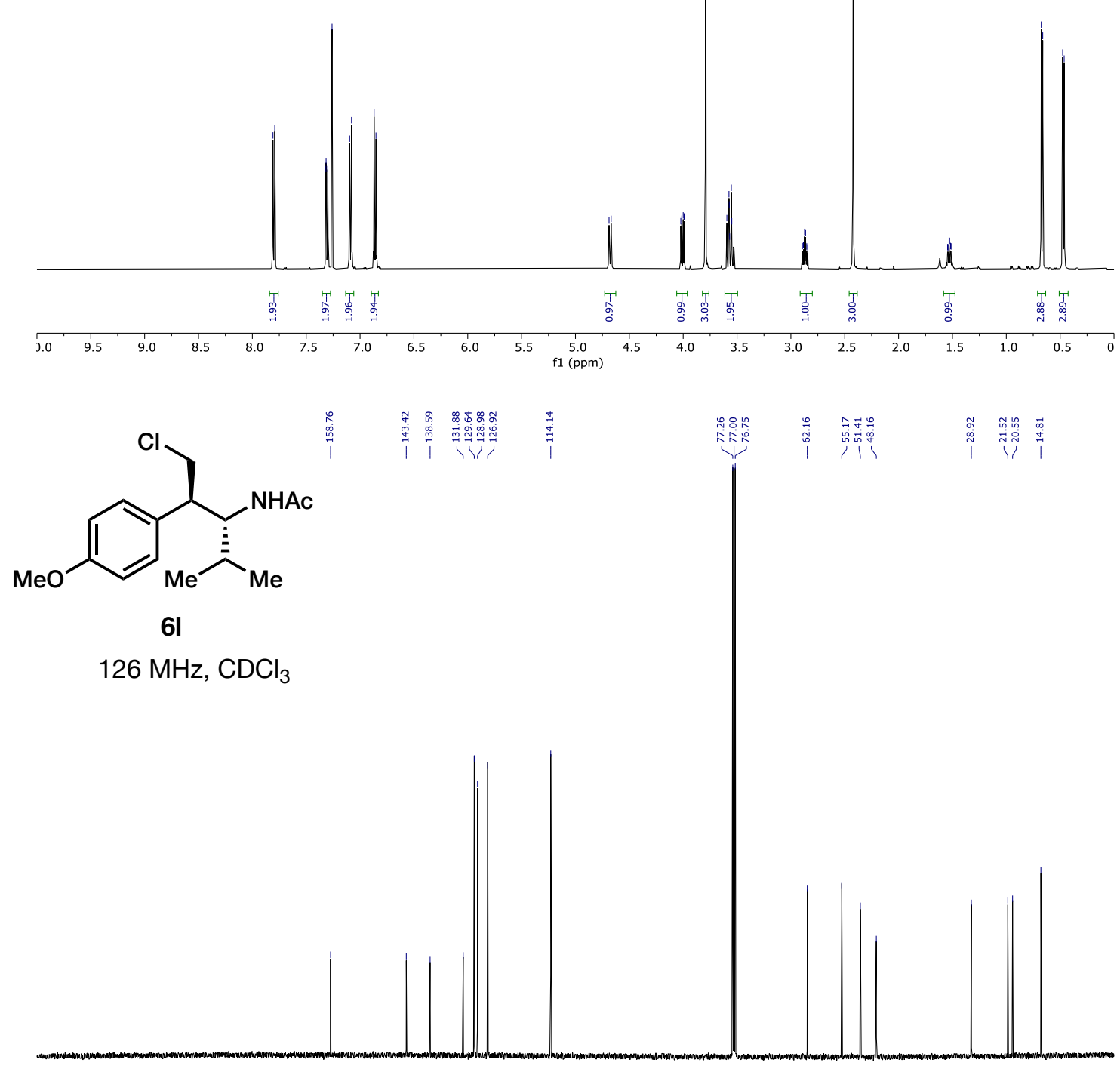
<smiles>COc1ccc([C@H](CCl)[C@H](CCCCCl)NC(=O)[O-])cc1</smiles>

$N$-((2R*,3R*)-1,7-Dichloro-2-(4-methoxyphenyl)heptan-3-yl)acetamide $6 \mathrm{~m}$

General Procedure D: Aziridine $5 \mathrm{~m}(89 \mathrm{mg}, 0.30 \mathrm{mmol})$ was employed. Purification of the residue by column chromatography (10-60\%, ethyl acetate in hexanes) afforded the title compound (76 $\mathrm{mg}, 76 \%)$ as a white solid.

${ }^{1} \mathrm{H}$ NMR $\left(500 \mathrm{MHz}, \mathrm{CDCl}_{3}\right) \delta 7.11(\mathrm{~d}, J=8.7 \mathrm{~Hz}, 2 \mathrm{H}), 6.89(\mathrm{~d}, J=8.6 \mathrm{~Hz}, 2 \mathrm{H}), 5.26(\mathrm{~d}, J=9.6$ $\mathrm{Hz}, 1 \mathrm{H}$ ), 4.27 (app. qd, $J=9.7,2.8 \mathrm{~Hz}, 1 \mathrm{H}$ ), 3.84 (dd, $J=11.1,5.6 \mathrm{~Hz}, 1 \mathrm{H}), 3.84(\mathrm{~s}, 3 \mathrm{H}), 3.69$ (dd, $J=11.1,8.6 \mathrm{~Hz}, 1 \mathrm{H}), 3.43(\mathrm{t}, J=6.5 \mathrm{~Hz}, 2 \mathrm{H}), 2.92$ (app td, $J=8.8,5.5 \mathrm{~Hz}, 1 \mathrm{H}), 2.04(\mathrm{~s}, 3 \mathrm{H})$, 1.76-1.68 (m, 1H), 1.62-1.55 (m, 1H), 1.52-1.38 (m, 2H), 1.35-1.28 (m, 1H), 1.19-1.08 (m, 1H);

${ }^{13} \mathrm{C}$ NMR $\left(126 \mathrm{MHz} \mathrm{CDCl}_{3}\right) \delta 170.1,158.9,131.0,129.2,114.2,55.2,52.7,51.6,46.9,44.7,31.9$, 31.4, 23.5, 22.8;

IR ( $\mathrm{NaCl}$, thin film) 3266, 2954, 1645, 1513, $1249 \mathrm{~cm}^{-1}$;

HRMS (ESI-TOF) $\mathrm{m} / \mathrm{z}$ calcd for $\mathrm{C}_{16} \mathrm{H}_{23} \mathrm{O}_{2} \mathrm{NCl}_{2} \mathrm{Na}(\mathrm{M}+\mathrm{Na})^{+}$: 354.0998, found 354.1000;

m. pt $83-86{ }^{\circ} \mathrm{C}$ 
<smiles>COc1ccc([C@H](CCl)[C@@H](CCCC(C)Cl)NC(C)(Cl)CCl)cc1</smiles>

$500 \mathrm{MHz}, \mathrm{CDCl}_{3}$
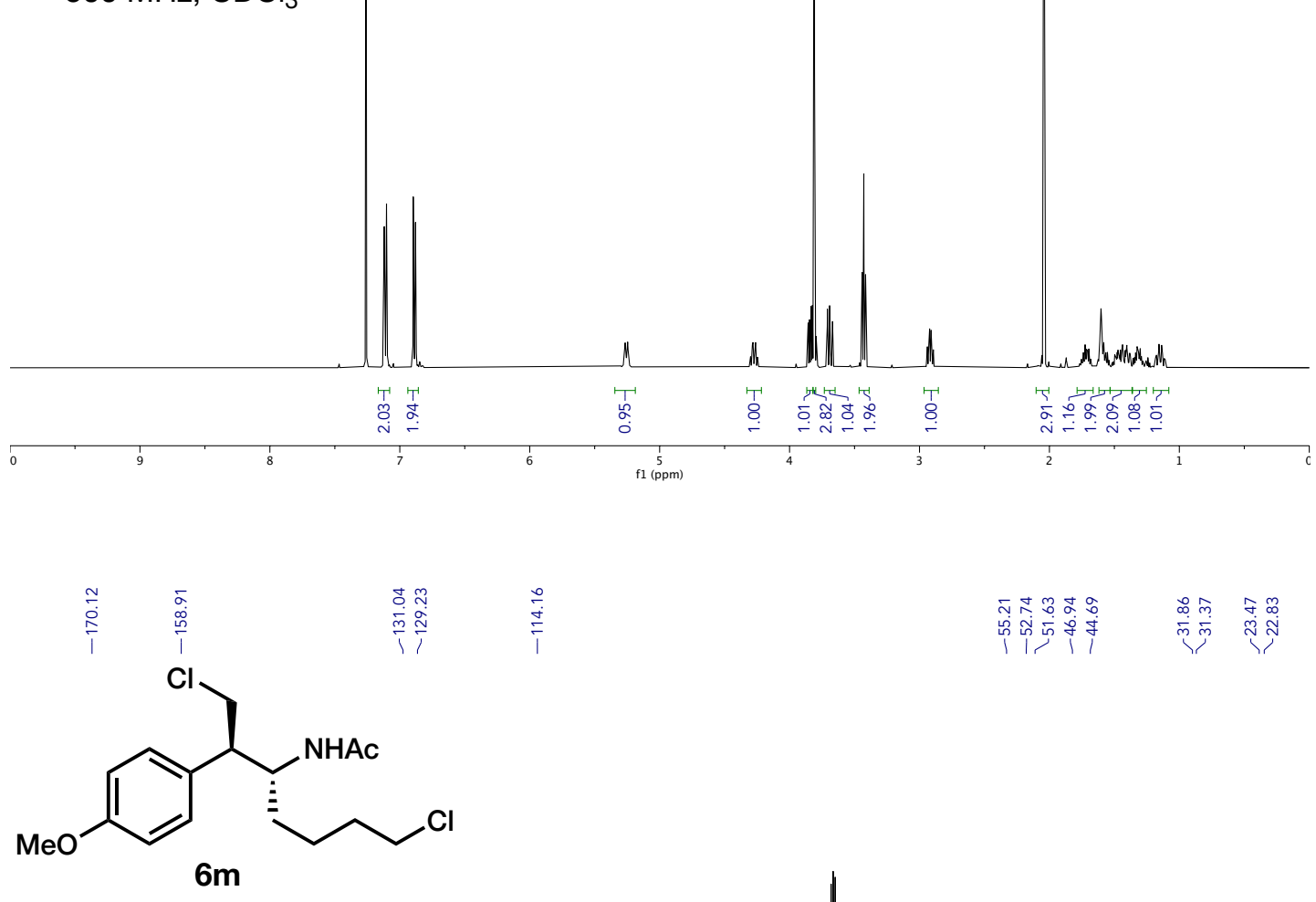

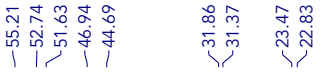

$126 \mathrm{MHz}, \mathrm{CDCl}_{3}$

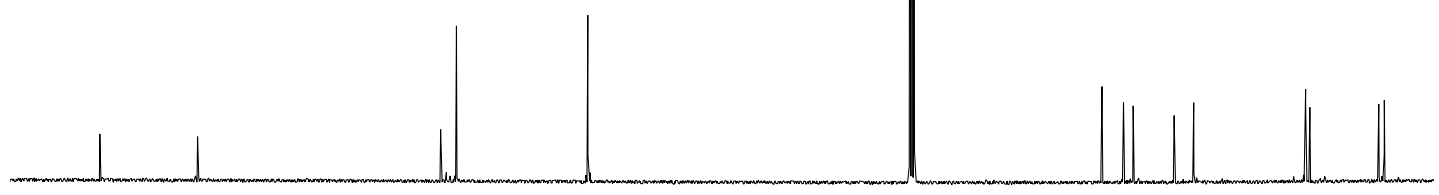

80

140

120

100
$f 1(p p m)$

60

40

20 
<smiles>COc1ccc([C@H](CCl)[C@H](CCCCC#N)NC(C)(F)F)cc1</smiles>

$N-\left(\left(2 R^{*}, 3 R^{*}\right)-1-C h l o r o-7-c y a n o-2-(4-m e t h o x y p h e n y l) h e p t a n-3-y l\right) a c e t a m i d e ~ 6 n$

General Procedure D: Aziridine 5n (86 mg, $0.30 \mathrm{mmol})$ was employed. Purification of the residue by column chromatography (10-60\%, ethyl acetate in hexanes) afforded the title compound (60 $\mathrm{mg}, 62 \%)$ as an orange oil.

${ }^{1} \mathrm{H}$ NMR $\left(500 \mathrm{MHz}, \mathrm{CDCl}_{3}\right) \delta 7.11(\mathrm{~d}, J=8.6 \mathrm{~Hz}, 2 \mathrm{H}), 6.89(\mathrm{~d}, J=8.6 \mathrm{~Hz}, 2 \mathrm{H}), 5.37(\mathrm{~d}, J=9.7$ $\mathrm{Hz}, 1 \mathrm{H}), 4.32-4.24(\mathrm{~m}, 1 \mathrm{H}), 3.83(\mathrm{dd}, J=11.1,5.6 \mathrm{~Hz}, 1 \mathrm{H}), 3.81(\mathrm{~s}, 3 \mathrm{H}), 3.68$ (dd, J = 11.2, 8.4 $\mathrm{Hz}, 1 \mathrm{H}), 2.91$ (app td, $J=8.7,5.6 \mathrm{~Hz}, 1 \mathrm{H}), 2.24(\mathrm{t}, J=7.1 \mathrm{~Hz}, 2 \mathrm{H}), 2.05(\mathrm{~s}, 3 \mathrm{H}), 1.67-1.58(\mathrm{~m}$, $1 \mathrm{H}), 1.51-1.32(\mathrm{~m}, 4 \mathrm{H}), 1.22-1.15(\mathrm{~m}, 1 \mathrm{H})$;

${ }^{13} \mathrm{C}$ NMR $\left(126 \mathrm{MHz}, \mathrm{CDCl}_{3}\right) \delta 170.2,159.0,130.9,129.2,119.4,114.2,55.2,52.6,51.3,46.9$, 31.4, 24.7, 24.6, 23.4, 16.9;

IR ( $\mathrm{NaCl}$, thin film) 3363, 2935, 2247, 1740, 1645, 1514, 1374, 1249, 1180, 1033, 833, $734 \mathrm{~cm}^{-1}$; HRMS (ESI-TOF) $m / z$ calcd for $\mathrm{C}_{17} \mathrm{H}_{23} \mathrm{O}_{2} \mathrm{~N}_{2} \mathrm{CINa}(\mathrm{M}+\mathrm{Na})^{+}: 345.1340$, found 345.1339. 

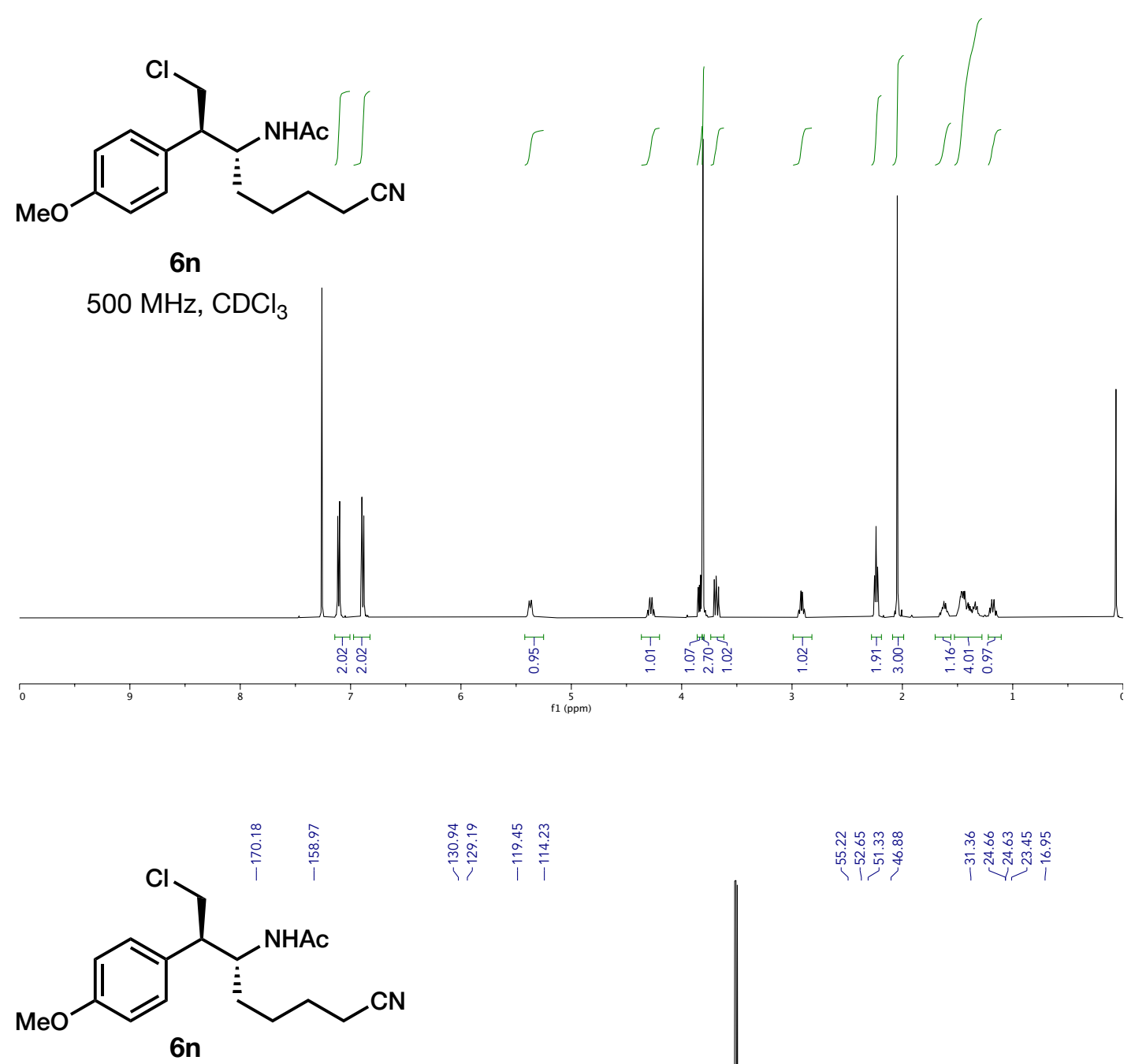

$126 \mathrm{MHz}, \mathrm{CDCl}_{3}$

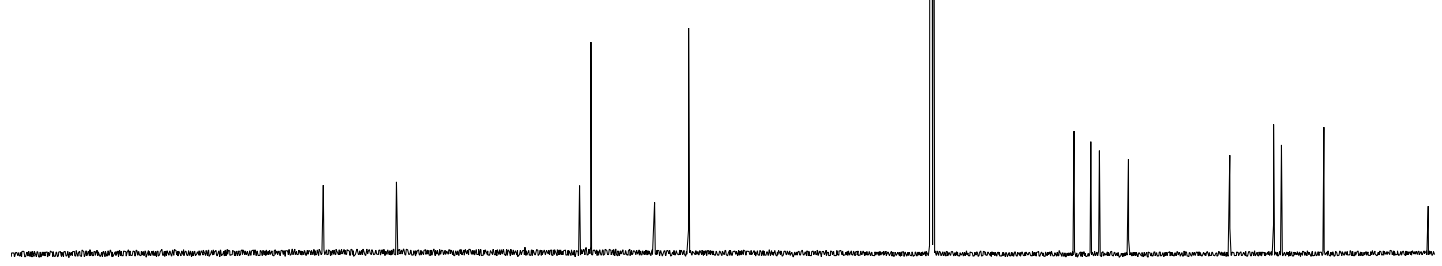




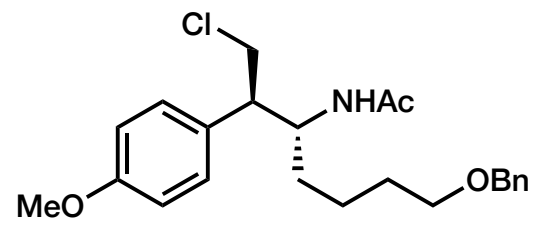

\section{$N-\left(\left(2 R^{*}, 3 R^{*}\right)-7-(B e n z y l o x y)-1-c h l o r o-2-(4-m e t h o x y p h e n y l) h e p t a n-3-y l\right) a c e t a m i d e 60$}

General Procedure D: Aziridine 5o $(110 \mathrm{mg}, 0.30 \mathrm{mmol})$ was employed. Purification of the residue by column chromatography (5-25\%, ethyl acetate in hexanes) afforded the title compound ( 87 $\mathrm{mg}, 72 \%)$ as a clear oil.

${ }^{1} \mathrm{H}$ NMR $\left(500 \mathrm{MHz}, \mathrm{CDCl}_{3}\right) \delta$ 7.33-7.26 (m, 5H), $7.10(\mathrm{~d}, J=8.7 \mathrm{~Hz}, 2 \mathrm{H}), 6.87(\mathrm{~d}, J=8.7 \mathrm{~Hz}, 2 \mathrm{H})$, $5.28(\mathrm{~d}, J=9.6 \mathrm{~Hz}, 1 \mathrm{H}), 4.43(\mathrm{~s}, 2 \mathrm{H}), 4.29-4.19(\mathrm{~m}, 1 \mathrm{H}), 3.86-3.78(\mathrm{~m}, 4 \mathrm{H}), 3.68(\mathrm{dd}, J=11.1$, $8.8 \mathrm{~Hz}, 1 \mathrm{H}), 3.37(\mathrm{t}, J=6.2 \mathrm{~Hz}, 2 \mathrm{H}), 2.95-2.87(\mathrm{~m}, 1 \mathrm{H}), 2.01(\mathrm{~s}, 3 \mathrm{H}), 1.60-1.50(\mathrm{~m}, 1 \mathrm{H}), 1.49-1.34$ $(\mathrm{m}, 3 \mathrm{H}), 1.32-1.20(\mathrm{~m}, 1 \mathrm{H}), 1.13(\mathrm{t}, \mathrm{J}=9.8 \mathrm{~Hz}, 1 \mathrm{H})$;

${ }^{13} \mathrm{C}$ NMR $\left(126 \mathrm{MHz}, \mathrm{CDCl}_{3}\right) \delta 170.1,158.8,138.4,131.2,129.3,128.4,127.7,127.6,114.1,72.9$, $69.9,55.2,52.8,52.0,47.0,31.8,29.2,23.4,22.4$;

IR ( $\mathrm{NaCl}$, thin film) 3255, 3030, 1644, 1583, 1454, $1303 \mathrm{~cm}^{-1}$;

HRMS (ESI-TOF) $\mathrm{m} / \mathrm{z}$ calcd for $\mathrm{C}_{23} \mathrm{H}_{30} \mathrm{CINO}_{3} \mathrm{Na}(\mathrm{M}+\mathrm{Na})^{+}:$426.1806, found 426.1818. 

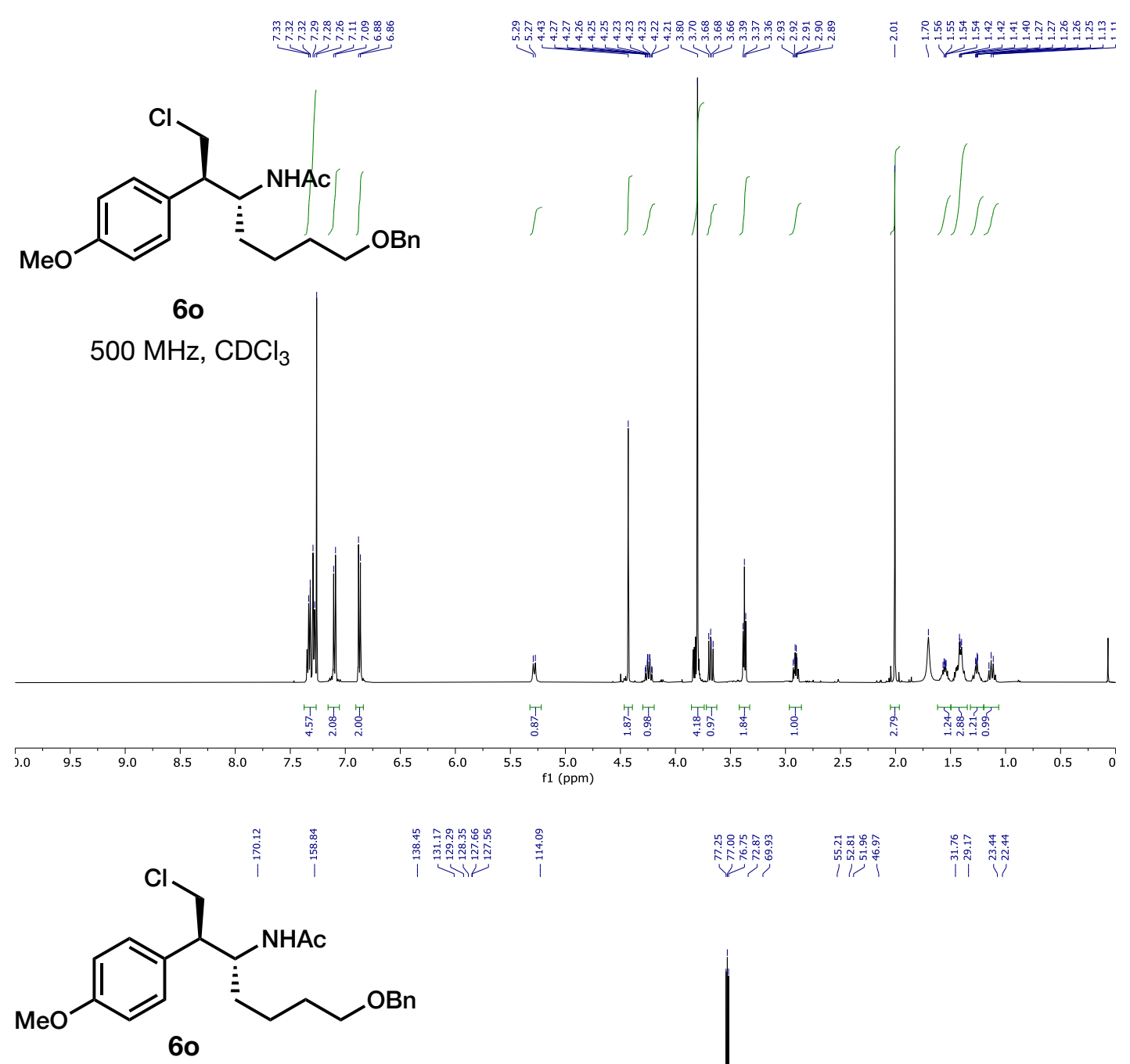

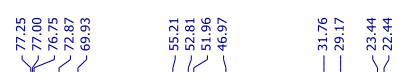

$126 \mathrm{MHz}, \mathrm{CDCl}_{3}$

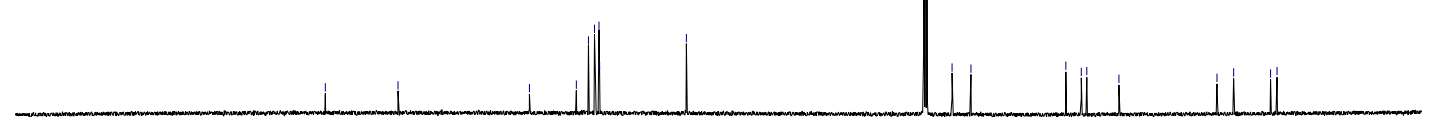

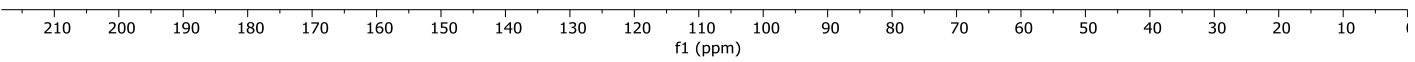


<smiles>COc1ccc([C@H](CCl)[C@H](N)CCCCNc2ccccc2)cc1</smiles>

$N-\left(\left(2 R^{*}, 3 R^{*}\right)-1-C h l o r o-7-(1,3-d i o x o i s o i n d o l i n-2-y l)-2-(4-m e t h o x y p h e n y l) h e p t a n-3-\right.$ yl)acetamide $6 p$

General Procedure D: Aziridine 5p (122 mg, $0.30 \mathrm{mmol}$ ) was employed. Purification of the residue by column chromatography (5-35\%, ethyl acetate in hexanes) afforded the title compound (49 $\mathrm{mg}, 37 \%)$ as a white solid.

${ }^{1} \mathrm{H}$ NMR $\left(500 \mathrm{MHz}, \mathrm{CDCl}_{3}\right) \delta 7.82(\mathrm{dd}, J=5.4,3.1 \mathrm{~Hz}, 2 \mathrm{H}), 7.71$ (dd, $\left.J=5.4,3.1 \mathrm{~Hz}, 2 \mathrm{H}\right), 7.11$ $(\mathrm{d}, J=8.7 \mathrm{~Hz}, 2 \mathrm{H}), 6.87(\mathrm{~d}, J=8.6 \mathrm{~Hz}, 2 \mathrm{H}), 5.53(\mathrm{~d}, J=9.3 \mathrm{~Hz}, 1 \mathrm{H}), 4.16(\mathrm{dt}, J=9.5,2.5 \mathrm{~Hz}$, $1 \mathrm{H}), 3.85$ (dd, $J=11.1,5.2 \mathrm{~Hz}, 1 \mathrm{H}), 3.80(\mathrm{~s}, 3 \mathrm{H}), 3.66(\mathrm{~s}, 1 \mathrm{H}), 3.61$ (ddd, $J=7.5,6.0,2.4 \mathrm{~Hz}$, 2H), $2.89(\mathrm{td}, J=9.1,5.2 \mathrm{~Hz}, 1 \mathrm{H}), 2.01(\mathrm{~s}, 3 \mathrm{H}), 1.70-1.62(\mathrm{~m}, 1 \mathrm{H}), 1.52-1.32(\mathrm{~m}, 2 \mathrm{H}), 1.25-1.22$ $(\mathrm{m}, 3 \mathrm{H})$;

${ }^{13} \mathrm{C}$ NMR $\left(126 \mathrm{MHz}, \mathrm{CDCl}_{3}\right) \delta 170.3,168.6,158.8,134.0,132.0,131.3,129.2,123.2,114.1,55.2$, $53.3,52.0,47.1,36.9,31.2,27.8,23.3,22.4$;

IR (NaCl, thin film) 3270, 2944, 1711, 1650, 1513, 1438, $1372 \mathrm{~cm}^{-1}$;

HRMS (ESI-TOF) $\mathrm{m} / \mathrm{z}$ calcd for $\mathrm{C}_{24} \mathrm{H}_{27} \mathrm{CIN}_{2} \mathrm{O}_{4} \mathrm{Na}(\mathrm{M}+\mathrm{Na})^{+}:$465.1552, found 465.1574.

m. pt $114-117^{\circ} \mathrm{C}$ 

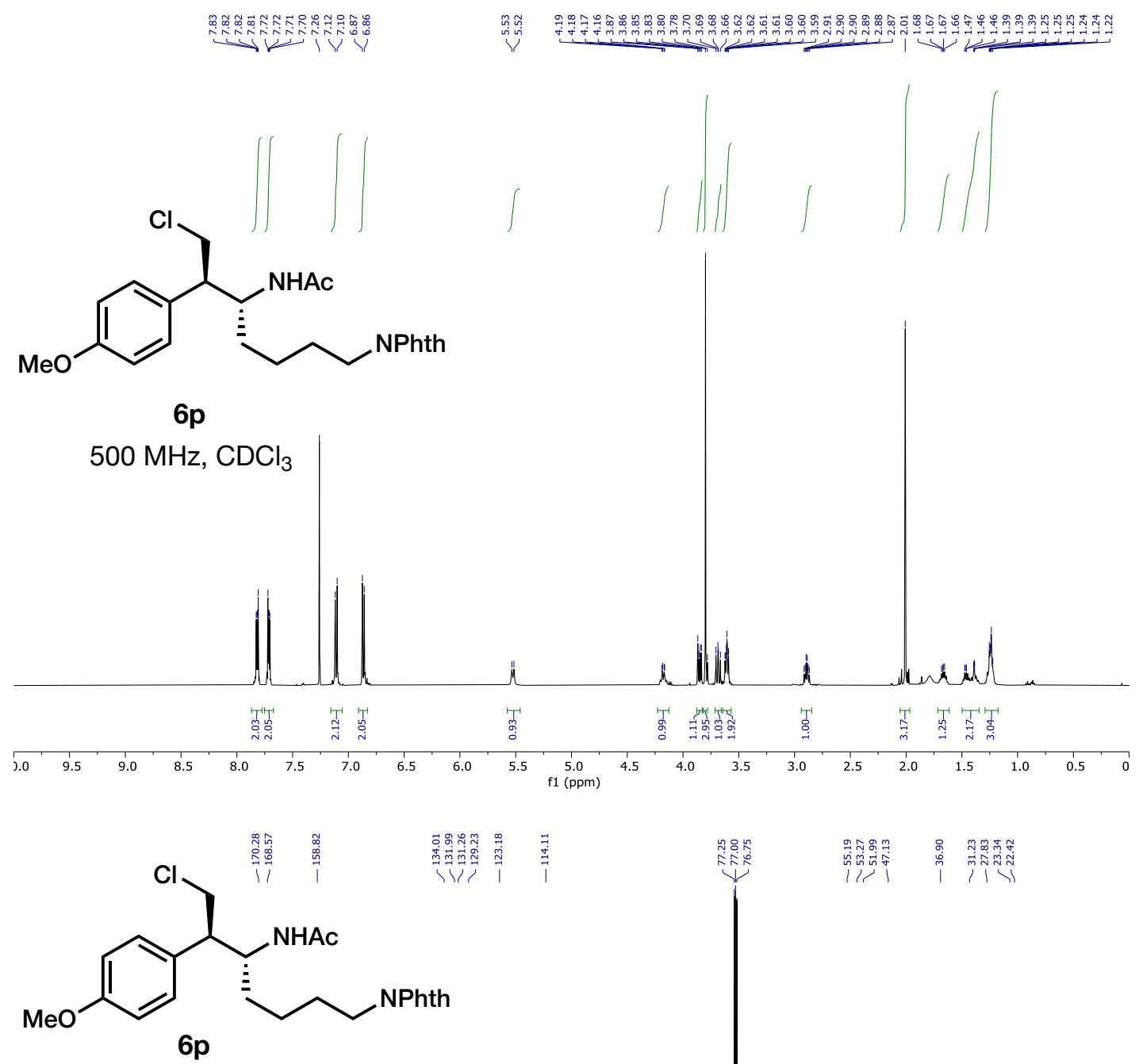

$126 \mathrm{MHz}, \mathrm{CDCl}_{3}$

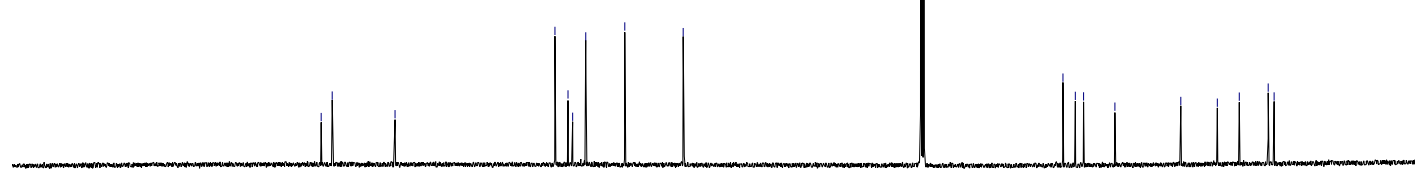

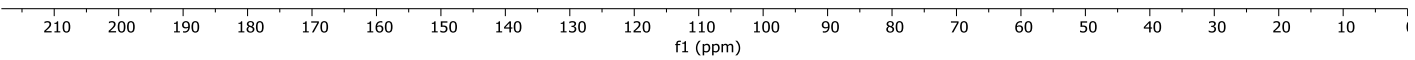


<smiles>CN[C@H](CC1CC[S+](=S)CC1)[C@H](CCl)c1ccc(OC)cc1</smiles>

$N-\left(\left(2 R^{*}, 3 R^{*}\right)-4-C h l o r o-3-(4-m e t h o x y p h e n y l)-1-(1-t o s y l p i p e r i d i n-4-y l) b u t a n-2-y l\right) a c e t a m i d e$ 6q

General Procedure D: Aziridine 5q (137 mg, $0.30 \mathrm{mmol})$ was employed. Purification of the residue by column chromatography (15-65\%, ethyl acetate in hexanes) afforded the title compound (79 $\mathrm{mg}, 53 \%)$ as an orange oil.

${ }^{1} \mathrm{H}$ NMR $\left(500 \mathrm{MHz}, \mathrm{CDCl}_{3}\right) \delta 7.57(\mathrm{~d}, J=8.3 \mathrm{~Hz}, 2 \mathrm{H}), 7.29(\mathrm{~d}, J=8.1 \mathrm{~Hz}, 2 \mathrm{H}), 7.04(\mathrm{~d}, J=8.7$ $\mathrm{Hz}, 2 \mathrm{H}), 6.86(\mathrm{~d}, J=8.6 \mathrm{~Hz}, 2 \mathrm{H}), 5.44(\mathrm{~d}, J=9.9 \mathrm{~Hz}, 1 \mathrm{H}), 4.34-4.28(\mathrm{~m}, 1 \mathrm{H}), 3.79(\mathrm{~s}, 3 \mathrm{H}), 3.78-$ $3.76(\mathrm{~m}, 1 \mathrm{H}), 3.67-3.61(\mathrm{~m}, 3 \mathrm{H}), 2.82(\mathrm{app} \mathrm{td}, J=8.7,5.8 \mathrm{~Hz}, 1 \mathrm{H}), 2.41(\mathrm{~s}, 3 \mathrm{H}), 2.12-2.06(\mathrm{~m}$, 2H), $1.94(\mathrm{~s}, 3 \mathrm{H}), 1.86(\mathrm{~d}, \mathrm{~J}=12.1 \mathrm{~Hz}, 1 \mathrm{H}), 1.46(\mathrm{~d}, \mathrm{~J}=12.3 \mathrm{~Hz}, 1 \mathrm{H}), 1.23-0.97(\mathrm{~m}, 5 \mathrm{H})$;

${ }^{13} \mathrm{C}$ NMR $\left(126 \mathrm{MHz}, \mathrm{CDCl}_{3}\right) \delta 170.0,158.9,143.4,132.8,130.8,129.6,129.2,127.6,114.1,55.2$, $52.9,48.9,46.7,46.3,46.2,39.1,32.2,31.7,30.2,23.3,21.4$;

IR ( $\mathrm{NaCl}$, thin film) 3363, 2932, 1651, 1514, 1445, $1249 \mathrm{~cm}^{-1}$;

HRMS (ESI-TOF) $\mathrm{m} / \mathrm{z}$ calcd for $\mathrm{C}_{25} \mathrm{H}_{33} \mathrm{O}_{4} \mathrm{~N}_{2} \mathrm{CISNa}(\mathrm{M}+\mathrm{Na})^{+}: 515.1742$, found 515.1748 . 

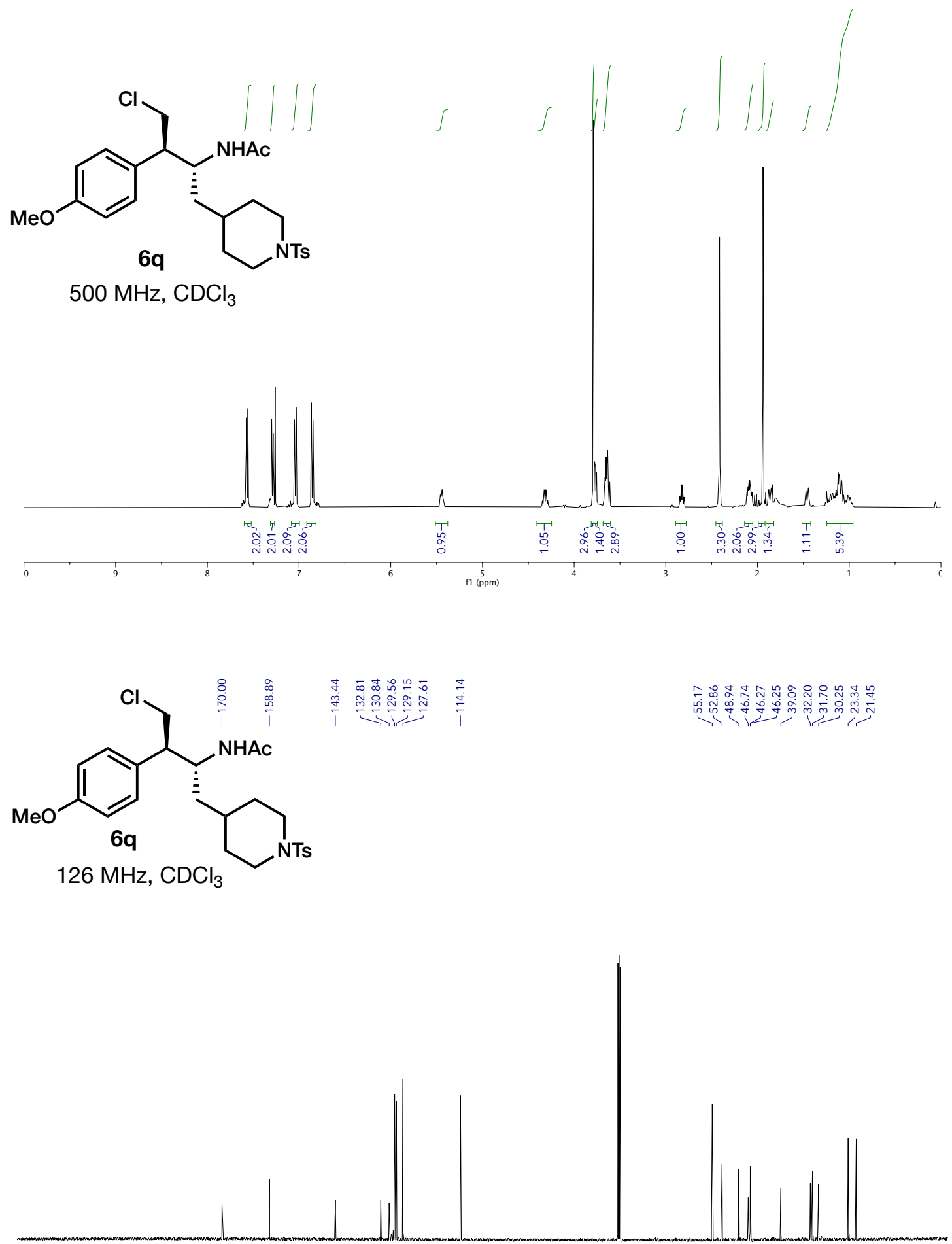

200

180

160

140

$120 \quad \mathrm{f} 1(\mathrm{ppm}) \quad 100$

80

60

40

$20 \quad c$ 


\section{Product derivatization reactions}<smiles>CCP[C@H]1[C@H](c2ccc(OC)cc2)CN1[As]</smiles>

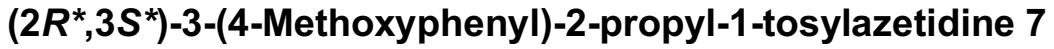

To a solution of phenethylamine $6 \mathrm{a}-\mathrm{Ts}(51 \mathrm{mg}, 0.13 \mathrm{mmol})$ in DMF $(2.0 \mathrm{~mL})$ was added potassium tert-butoxide (16 mg, $0.14 \mathrm{mmol}$ ) and sodium iodide $(4 \mathrm{mg}, 0.03 \mathrm{mmol}$ ). The mixture was stirred at $100{ }^{\circ} \mathrm{C}$ overnight via a heating block and then quenched with water $(10 \mathrm{~mL})$ and extracted with diethyl ether $(3 \times 5 \mathrm{~mL})$. The combined organic layers were washed with brine $(2 \times 10 \mathrm{~mL})$, dried with $\mathrm{MgSO}_{4}$ and concentrated in vacuo. Purification by column chromatography (4:1, hexanes:EtOAc) afforded the title compound (43 $\mathrm{mg}, 92 \%)$.

${ }^{1} \mathrm{H}$ NMR $\left(500 \mathrm{MHz}, \mathrm{CDCl}_{3}\right) \delta 7.79(\mathrm{~d}, J=8.3 \mathrm{~Hz}, 2 \mathrm{H}), 7.44(\mathrm{~d}, J=8.3,2 \mathrm{H}), 6.67(\mathrm{~d}, J=8.7 \mathrm{~Hz}$, $2 \mathrm{H}), 6.61(\mathrm{~d}, J=8.7 \mathrm{~Hz}, 2 \mathrm{H}), 4.02(\mathrm{t}, J=8.3 \mathrm{~Hz}, 1 \mathrm{H}), 3.83(\mathrm{dt}, J=8.1,4.0 \mathrm{~Hz}, 1 \mathrm{H}), 3.74(\mathrm{~s}, 3 \mathrm{H})$, $3.52(\mathrm{t}, J=8.1 \mathrm{~Hz}, 1 \mathrm{H}), 3.24(\mathrm{q}, J=5.1 \mathrm{~Hz}, 1 \mathrm{H}), 2.52(\mathrm{~s}, 3 \mathrm{H}), 1.96-1.86(\mathrm{~m}, 1 \mathrm{H}), 1.82-1.71(\mathrm{~m}$, $1 \mathrm{H}), 1.36-1.16(\mathrm{~m}, 2 \mathrm{H}), 0.84(\mathrm{t}, J=7.3 \mathrm{~Hz}, 3 \mathrm{H})$.

${ }^{13} \mathrm{C}$ NMR $\left(126 \mathrm{MHz}, \mathrm{CDCl}_{3}\right) \delta 158.6,144.0,132.1,131.9,129.8,128.6,128.2,113.9,71.9,55.3$, $55.2,40.5,38.2,21.6,17.6,14.0$;

IR (NaCl, thin film) 2873, 1515, 1465, 1344, $1249 \mathrm{~cm}^{-1}$;

HRMS (ESI-TOF) $\mathrm{m} / \mathrm{z}$ calcd for $\mathrm{C}_{20} \mathrm{H}_{25} \mathrm{NO}_{3} \mathrm{SNa}(\mathrm{M}+\mathrm{Na})^{+}: 382.1447$, found 382.1449.

The relative stereochemistry was determined through nOe analysis, indicated below.

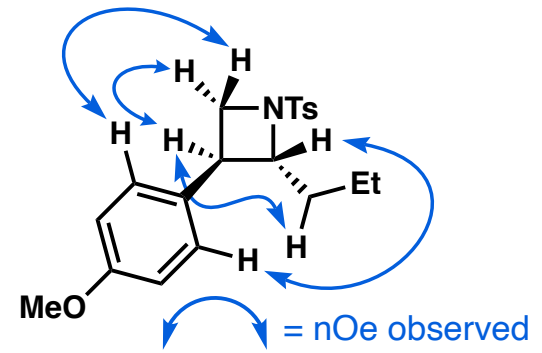



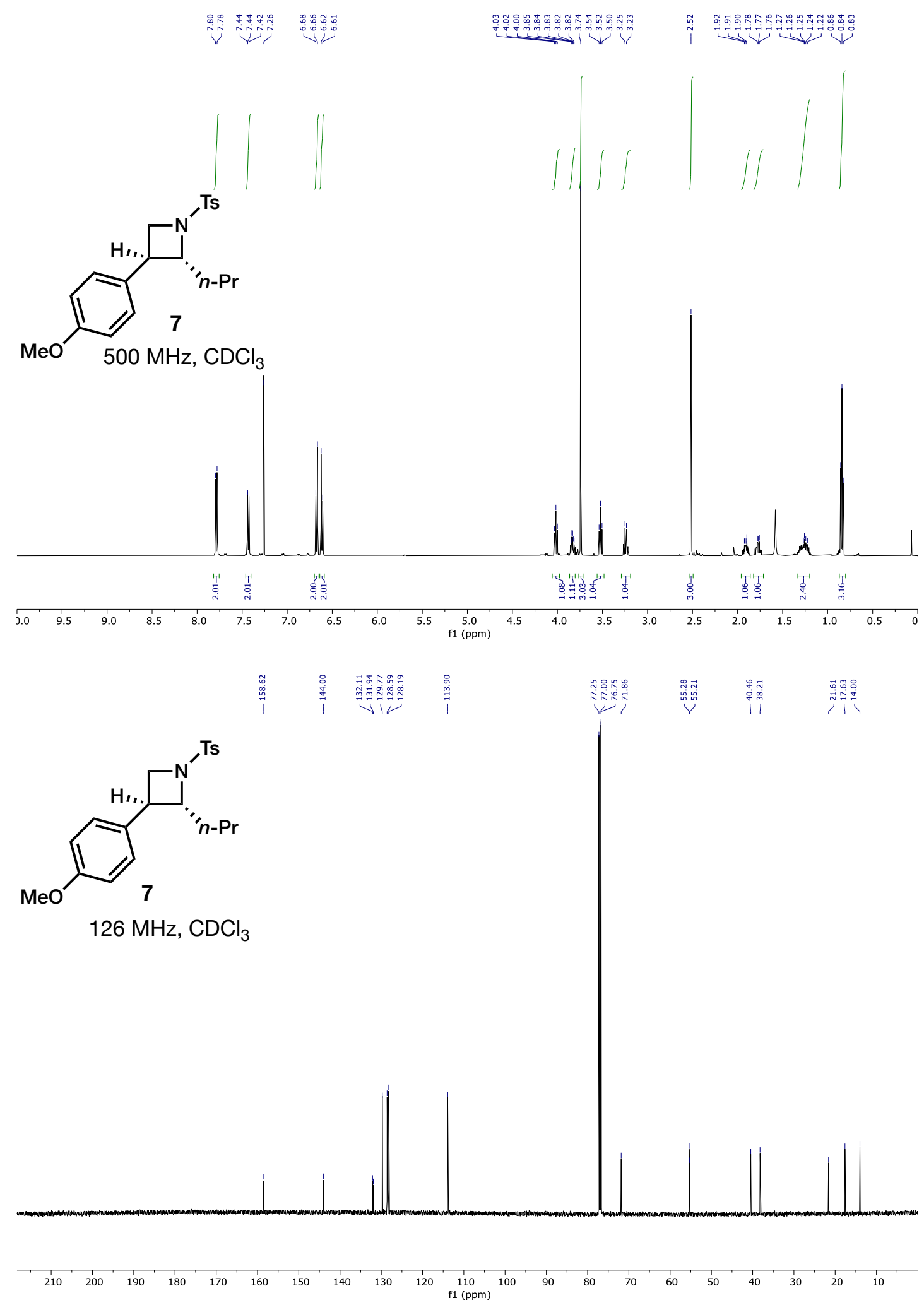
<smiles>[13CH3][C@@H]1[C@@H](C(=O)O)CN1[13CH3]</smiles>

\section{$\left(2 R^{*}, 3 S^{*}\right)-2-P r o p y l-1-t o s y l a z e t i d i n e-3-c a r b o x y l i c$ acid 8}

To a solution of azetidine $7(43 \mathrm{mg}, 0.12 \mathrm{mmol})$ in $\mathrm{CCl}_{4}(1.0 \mathrm{~mL}), \mathrm{MeCN}(1.0 \mathrm{~mL})$ and water (1.5 $\mathrm{mL}$ ) was added ruthenium trichloride $(6 \mathrm{mg}, 0.03 \mathrm{mmol})$ and periodic acid $(0.47 \mathrm{~g}, 1.76 \mathrm{mmol})$. The mixture was stirred overnight at room temperature. Upon completion, diethyl ether $(5 \mathrm{~mL})$ was added and stirred for 15 minutes before water $(10 \mathrm{~mL})$ was added. The product was extracted with diethyl ether $(3 \times 5 \mathrm{~mL})$, and the combined layers were washed with brine $(1 \times 10 \mathrm{~mL})$, dried with $\mathrm{MgSO}_{4}$ and concentrated in vacuo to afford the title compound (25 $\left.\mathrm{mg}, 70 \%\right)$.

${ }^{1} \mathrm{H}$ NMR $\left(500 \mathrm{MHz}, \mathrm{CDCl}_{3}\right) \delta 7.73(\mathrm{~d}, J=8.3 \mathrm{~Hz}, 2 \mathrm{H}), 7.38(\mathrm{~d}, J=8.0 \mathrm{~Hz}, 2 \mathrm{H}), 4.04$ (ddd, $J=8.4$, 7.3, $4.6 \mathrm{~Hz}, 1 \mathrm{H}), 3.86(\mathrm{t}, J=8.4 \mathrm{~Hz}, 1 \mathrm{H}), 3.75(\mathrm{t}, J=8.0 \mathrm{~Hz}, 1 \mathrm{H}), 3.04-2.96(\mathrm{~m}, 1 \mathrm{H}), 2.47(\mathrm{~s}, 3 \mathrm{H})$, 1.95-1.85 (m, 1H), 1.76 (ddd, $J=10.4,5.2,2.6 \mathrm{~Hz}, 1 \mathrm{H}), 1.50-1.29(\mathrm{~m}, 3 \mathrm{H}), 0.95$ (t, $J=7.4 \mathrm{~Hz}$, $3 \mathrm{H})$;

${ }^{13} \mathrm{C}$ NMR $\left(126 \mathrm{MHz}_{\mathrm{CDCl}}\right)$ $\delta$ 176.3, 144.3, 131.8, 129.7, 128.3, 66.4, 49.2, 38.1, 37.7, 21.6, 17.3, 13.8;

IR ( $\mathrm{NaCl}$, thin film) 3245, 2874, 1713, 1598, 1465, $1341 \mathrm{~cm}^{-1}$;

HRMS (ESI-TOF) $\mathrm{m} / \mathrm{z}$ calcd for $\mathrm{C}_{14} \mathrm{H}_{19} \mathrm{NO}_{4} \mathrm{SNa}(\mathrm{M}+\mathrm{Na})^{+}: 320.0927$, found 320.0940 . 

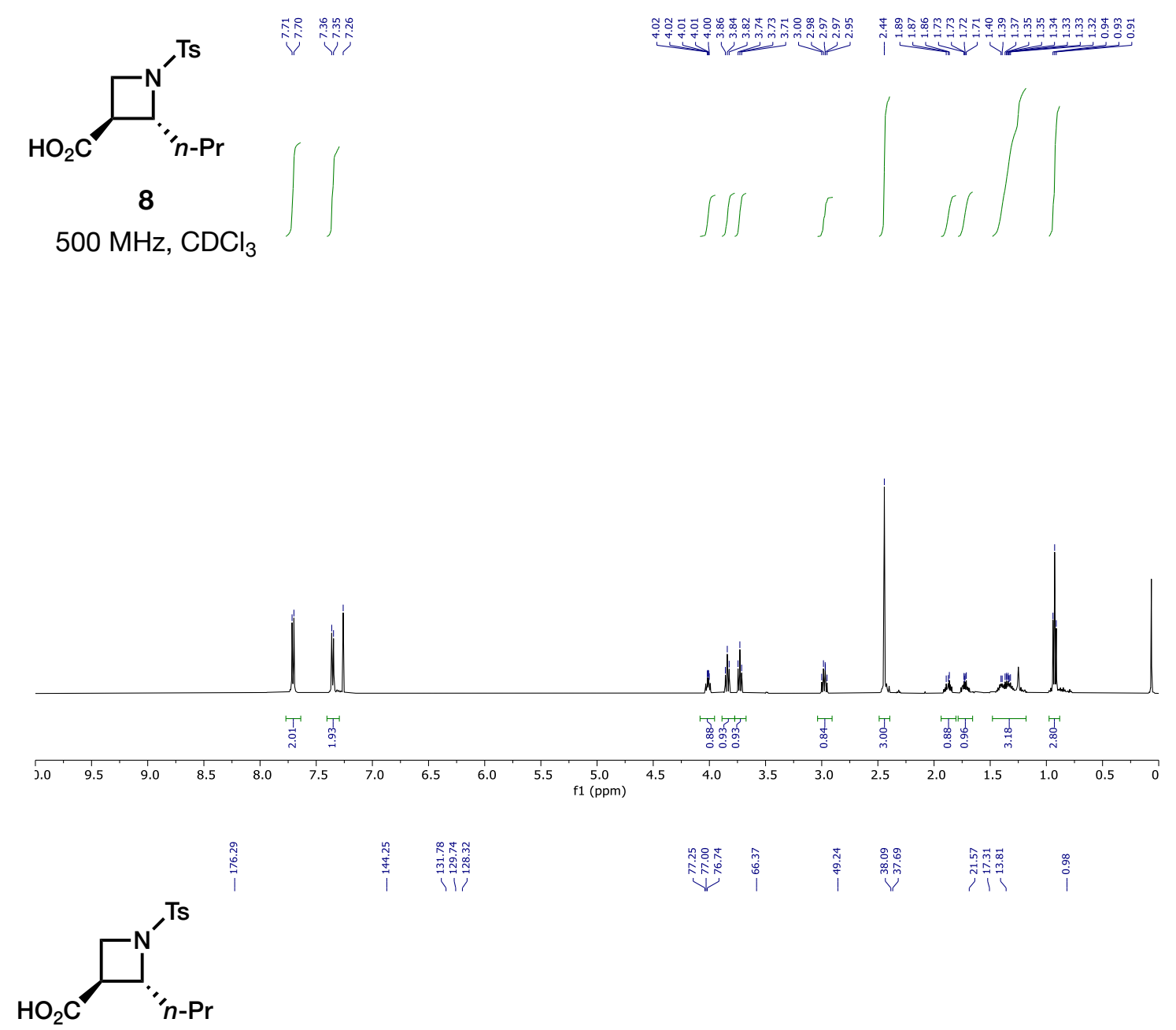

8

$126 \mathrm{MHz}, \mathrm{CDCl}_{3}$

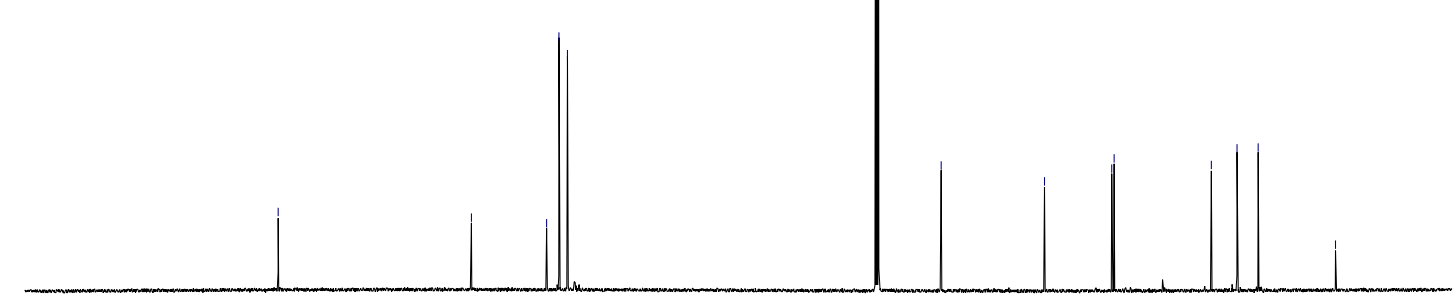

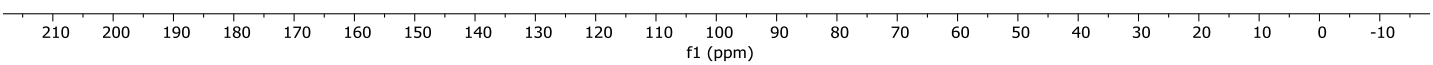


<smiles>CCCC[C@@H]1N=C(C)c2cc(OC)c(OC)cc2[C@H]1CCl</smiles>

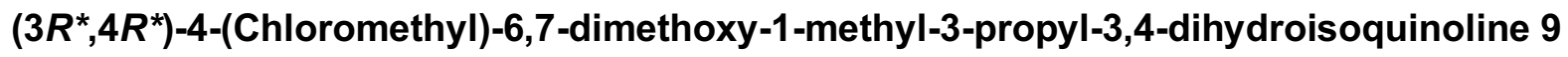

To a solution of phenethylamine $6 \mathrm{e}(60 \mathrm{mg}, 0.19 \mathrm{mmol})$ in toluene $(1.2 \mathrm{~mL})$ was added $\mathrm{POCl}_{3}(43$ $\mu \mathrm{L}, 0.46 \mathrm{mmol}$ ). The mixture was heated to $105^{\circ} \mathrm{C}$ for 3 hours via a heating block and then cooled to room temperature, concentrated in vacuo, and $10 \%$ aqueous $\mathrm{NaOH}$ was added until $\mathrm{pH} 9$ was achieved. The reaction was extracted with $\mathrm{CH}_{2} \mathrm{Cl}_{2}(3 \times 5 \mathrm{~mL})$, and the organic layers were combined, washed with brine $(5 \mathrm{~mL})$, dried with $\mathrm{MgSO}_{4}$, and concentrated in vacuo. The residue was purified by column chromatography $\left(2 \% \mathrm{MeOH}\right.$ in $\left.\mathrm{CH}_{2} \mathrm{Cl}_{2}\right)$ to afford the title compound (35 $\mathrm{mg}, 62 \%)$.

${ }^{1} \mathrm{H}$ NMR $\left(500 \mathrm{MHz}, \mathrm{CDCl}_{3}\right) \delta 7.00(\mathrm{~s}, 1 \mathrm{H}), 6.83(\mathrm{~s}, 1 \mathrm{H}), 3.94(\mathrm{~s}, 3 \mathrm{H}), 3.91(\mathrm{~s}, 3 \mathrm{H}), 3.77$ (dd, $J=$ 11.0, 4.3 Hz, 1H), 3.44-3.38 (m, 1H), $3.34(\mathrm{t}, J=10.7 \mathrm{~Hz}, 1 \mathrm{H}), 2.84(\mathrm{dt}, J=9.9,4.5 \mathrm{~Hz}, 1 \mathrm{H}), 2.39$ $(\mathrm{d}, J=2.4 \mathrm{~Hz}, 3 \mathrm{H}), 1.96-1.89(\mathrm{~m}, 1 \mathrm{H}), 1.78-1.70(\mathrm{~m}, 1 \mathrm{H}), 1.68-1.60(\mathrm{~m}, 1 \mathrm{H}), 1.53-1.44(\mathrm{~m}, 1 \mathrm{H})$, $1.00(\mathrm{t}, J=7.3 \mathrm{~Hz}, 3 \mathrm{H})$;

${ }^{13} \mathrm{C}$ NMR $\left(126 \mathrm{MHz}, \mathrm{CDCl}_{3}\right) \delta 163.9,150.5,148.2,132.7,121.6,112.2,108.8,59.2,56.1,56.0$, 43.5, 41.8, 35.5, 23.0, 19.9, 14.0;

IR ( $\mathrm{NaCl}$, thin film) 2932,, 1604, 1515, 1464, 1347, $1269 \mathrm{~cm}^{-1}$;

HRMS (ESI-TOF) $\mathrm{m} / \mathrm{z}$ calcd for $\mathrm{C}_{16} \mathrm{H}_{22} \mathrm{O}_{2} \mathrm{NClH}(\mathrm{M}+\mathrm{H})^{+}:$296.1412, found 296.296.1416. 

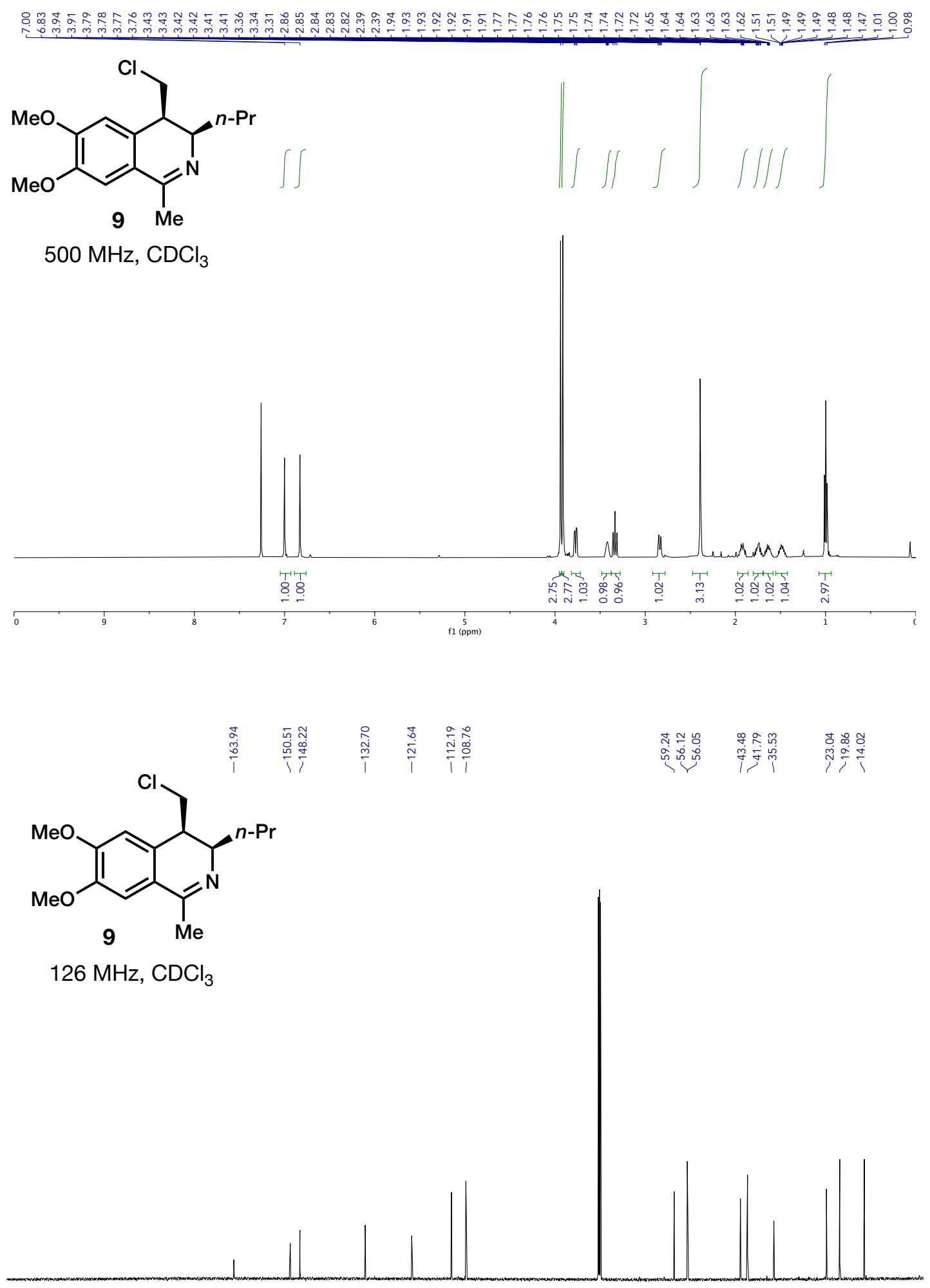
<smiles>COc1ccc2c(c1)C[NH2+][C@@H](PC)[C@H]2CCl</smiles>

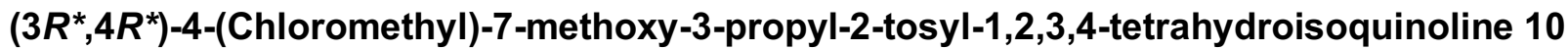

To a solution of phenethylamine $6 \mathbf{6}-\mathrm{Ts}(59 \mathrm{mg}, 0.15 \mathrm{mmol})$ in acetic acid $(0.5 \mathrm{~mL})$ and trifluoroacetic acid $(0.12 \mathrm{~mL})$ was added paraformaldehyde $(9 \mathrm{mg}, 0.30 \mathrm{mmol})$ in one portion. The mixture was heated at $100{ }^{\circ} \mathrm{C}$ for four hours via a heating block. The reaction was quenched with water $(1 \mathrm{~mL})$ and extracted with $\mathrm{CH}_{2} \mathrm{Cl}_{2}(3 \times 5 \mathrm{~mL})$. The organic layer was washed with $\mathrm{Na}_{2} \mathrm{CO}_{3}$ $(5 \mathrm{~mL})$ and brine $(5 \mathrm{~mL})$, dried with $\mathrm{MgSO}_{4}$, and concentrated in vacuo. The residue was purified by column chromatography ( $0-10 \%$, ethyl acetate in hexanes) to afford the title compound (34 $\mathrm{mg}, 56 \%)$.

${ }^{1} \mathrm{H}$ NMR $\left(500 \mathrm{MHz}, \mathrm{CDCl}_{3}\right) \delta 7.71(\mathrm{~d}, J=8.4 \mathrm{~Hz}, 2 \mathrm{H}), 7.23(\mathrm{~d}, J=8.1 \mathrm{~Hz}, 2 \mathrm{H}), 6.98(\mathrm{~d}, J=8.6$ $\mathrm{Hz}, 1 \mathrm{H}), 6.72(\mathrm{dd}, J=8.7,2.7 \mathrm{~Hz}, 1 \mathrm{H}), 6.63(\mathrm{~d}, J=2.7 \mathrm{~Hz}, 1 \mathrm{H}), 4.66(\mathrm{~d}, J=17.2 \mathrm{~Hz}, 1 \mathrm{H}), 4.53$ (dt, $J=11.2,4.1 \mathrm{~Hz}, 1 \mathrm{H}), 4.33(\mathrm{~d}, J=16.9 \mathrm{~Hz}, 1 \mathrm{H}), 4.09(\mathrm{dd}, J=11.2,5.5 \mathrm{~Hz}, 1 \mathrm{H}), 3.77(\mathrm{~s}, 3 \mathrm{H})$, $3.46(\mathrm{t}, J=10.8 \mathrm{~Hz}, 1 \mathrm{H}), 2.90(\mathrm{dt}, J=10.5,5.2 \mathrm{~Hz}, 1 \mathrm{H}), 2.38(\mathrm{~s}, 3 \mathrm{H}), 1.51-1.35(\mathrm{~m}, 2 \mathrm{H}), 1.26-$ $1.15(\mathrm{~m}, 1 \mathrm{H}), 1.13-1.03(\mathrm{~m}, 1 \mathrm{H}), 0.89(\mathrm{t}, J=7.4 \mathrm{~Hz}, 3 \mathrm{H})$;

${ }^{13} \mathrm{C}$ NMR $\left(126 \mathrm{MHz}, \mathrm{CDCl}_{3}\right) \delta 158.2,143.2,137.4,133.0,129.7,127.0,126.3,125.3,112.9$, 111.1, 55.2, 53.1, 44.0, 43.8, 41.2, 27.7, 21.5, 19.1, 13.8;

IR ( $\mathrm{NaCl}$, thin film) 2959, 1613, 1506, 1458, 1344, $1161 \mathrm{~cm}^{-1}$;

HRMS $\left(\mathrm{Cl}^{+}\right) \mathrm{m} / \mathrm{z}$ calcd for $\mathrm{C}_{21} \mathrm{H}_{26} \mathrm{O}_{3} \mathrm{NCISNH}_{4}\left(\mathrm{M}+\mathrm{NH}_{4}\right)^{+}: 425.1660$, found 425.1658 . 

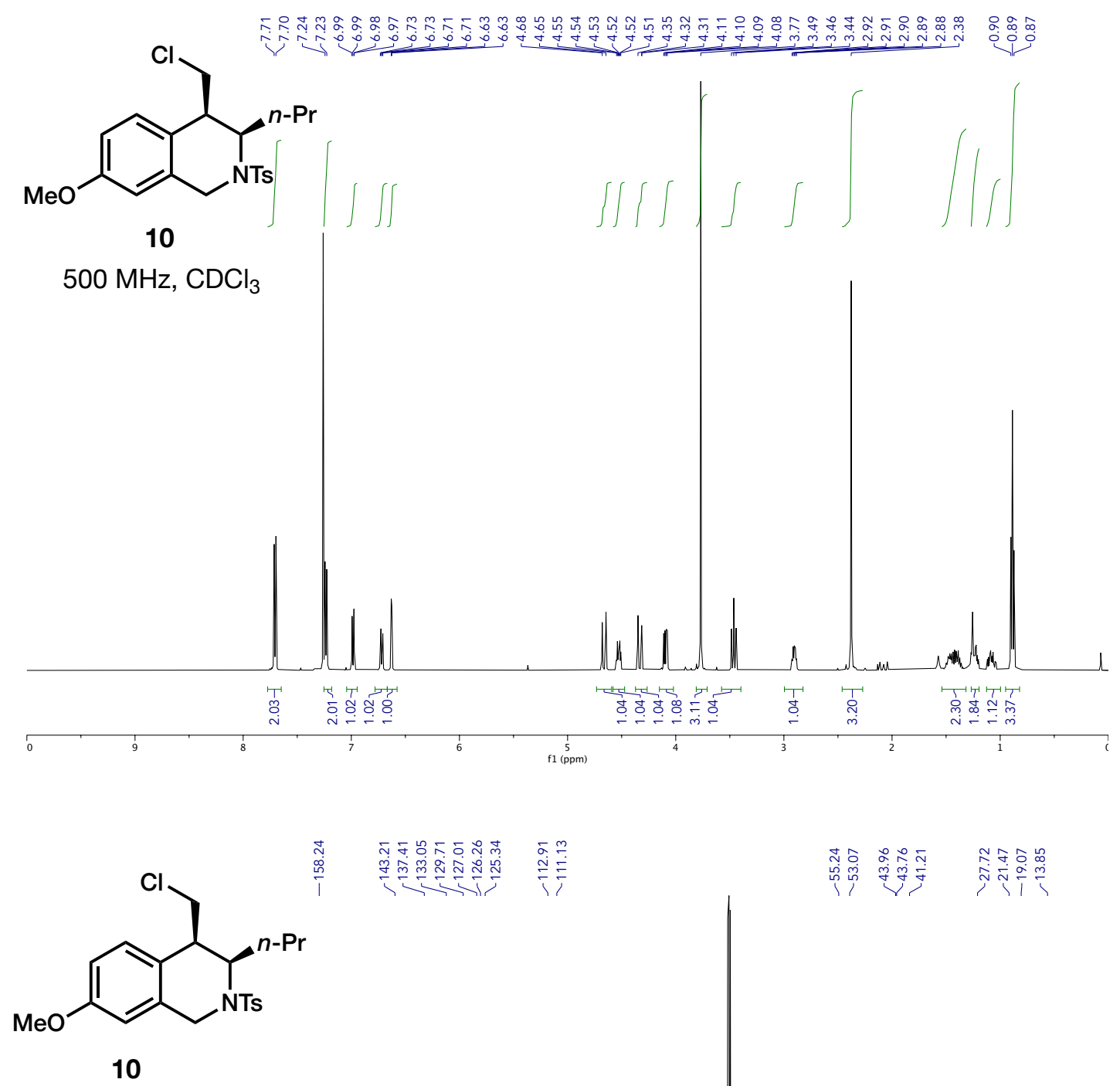

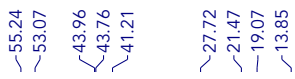

$126 \mathrm{MHz}, \mathrm{CDCl}_{3}$

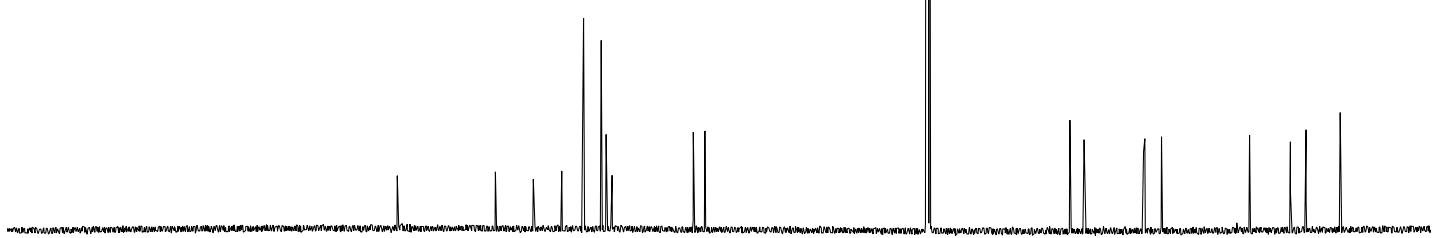




\section{Single Crystal X-ray Analysis of 6b}

Crystal grown via dissolving in minimum amount of $\mathrm{CH}_{2} \mathrm{Cl}_{2}$ and then layering with hexanes and cooling to $0^{\circ} \mathrm{C}$.

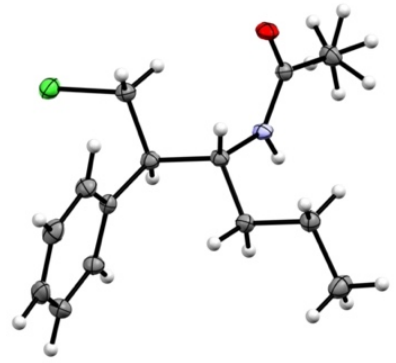

Table S1: Crystal data and structure refinement for $\mathbf{6 b}$ (ellipsoids at 50\% probability)

\begin{tabular}{|c|c|c|}
\hline CCDC Entry: & \multicolumn{2}{|l|}{2121218} \\
\hline Empirical formula & \multicolumn{2}{|l|}{$\mathrm{C}_{14} \mathrm{H}_{20} \mathrm{CINO}$} \\
\hline Formula weight & \multicolumn{2}{|l|}{253.76} \\
\hline Temperature & \multicolumn{2}{|l|}{$126(2) \mathrm{K}$} \\
\hline Wavelength & \multicolumn{2}{|l|}{$1.54178 \AA$} \\
\hline Crystal system & \multicolumn{2}{|l|}{ Orthorhombic } \\
\hline Space group & \multicolumn{2}{|l|}{ Pbca } \\
\hline \multirow[t]{3}{*}{ Unit cell dimensions } & $a=9.8026(6) \AA$ & $\alpha=90^{\circ}$ \\
\hline & $b=14.4768(8) \AA$ & $\beta=90^{\circ}$ \\
\hline & $c=18.9939(11) \AA$ & $\gamma=90^{\circ}$ \\
\hline Volume & \multicolumn{2}{|l|}{$2695.4(3) \AA^{3}$} \\
\hline Z & \\
\hline Density (calculated) & \multicolumn{2}{|l|}{$1.251 \mathrm{Mg} / \mathrm{m}^{3}$} \\
\hline Absorption coefficient & \multicolumn{2}{|l|}{$2.371 \mathrm{~mm}^{-1}$} \\
\hline$F(000)$ & \multicolumn{2}{|l|}{1088} \\
\hline Crystal color, morphology & \multicolumn{2}{|l|}{ colorless, sheet } \\
\hline Crystal size & \multicolumn{2}{|l|}{$0.4 \times 0.2 \times 0.1 \mathrm{~mm}^{3}$} \\
\hline Theta range for data collection & \multicolumn{2}{|c|}{4.656 to $74.821^{\circ}$} \\
\hline Index ranges & \multicolumn{2}{|c|}{$-12 \leq h \leq 9,-18 \leq k \leq 17,-23 \leq l \leq 23$} \\
\hline Reflections collected & \multicolumn{2}{|c|}{32515} \\
\hline Independent reflections & \multicolumn{2}{|c|}{$2762[R(\mathrm{int})=0.0359]$} \\
\hline Observed reflections & \multicolumn{2}{|c|}{2648} \\
\hline Completeness to theta $=67.679 \infty$ & \multicolumn{2}{|c|}{$99.9 \%$} \\
\hline Absorption correction & \multicolumn{2}{|c|}{ Semi-empirical from equivalents } \\
\hline Max. and min. transmission & \multicolumn{2}{|c|}{0.740 and 0.300} \\
\hline Refinement method & \multicolumn{2}{|c|}{ Full-matrix least-squares on $F^{2}$} \\
\hline Data / restraints / parameters & \multicolumn{2}{|c|}{2762 / 0 / 157} \\
\hline Goodness-of-fit on $F^{2}$ & \multirow{2}{*}{\multicolumn{2}{|c|}{$\begin{array}{l}1.034 \\
R 1=0.0332, w R 2=0.0859\end{array}$}} \\
\hline Final $R$ indices $[/>2 \operatorname{sigma}(I)]$ & & \\
\hline$R$ indices (all data) & \multicolumn{2}{|c|}{$R 1=0.0343, w R 2=0.0869$} \\
\hline Extinction coefficient & \multicolumn{2}{|c|}{$\mathrm{n} / \mathrm{a}$} \\
\hline Largest diff. peak and hole & \multicolumn{2}{|c|}{0.311 and -0.199 e. $\AA^{-3}$} \\
\hline
\end{tabular}

$$
R_{1}=\Sigma|| F_{\mathrm{o}}|-| F_{\mathrm{C}} \| / \sum\left|F_{\mathrm{o}}\right|, w R 2=\left[\sum\left[w\left(F_{\mathrm{o}}^{2}-F_{\mathrm{C}}{ }^{2}\right)^{2}\right] / \sum\left[w\left(F_{\mathrm{o}}^{2}\right)^{2}\right]\right]^{1 / 2}
$$




\section{Computational methods, discussion, and results}

Stationary points were initially generated from a conformational search (low mode/mixed torsional) and minimized with the OPLS3e force field as implemented in MacroModel (version 10.1) within Maestro (version 9.4) in the Schrodinger ${ }^{4}$ software suite (release 2020-4). Structures calculated within $5 \mathrm{kcal} \mathrm{mol}^{-1}$ of the lowest energy conformer were considered further with Density Functional Theory (DFT) using Gaussian $16 .{ }^{5}$ All stationary points were calculated in the solution phase (using the IEF-PCM ${ }^{6}$ and $\mathrm{SMD}^{7}$ solvation models, solvent $=\mathrm{CH}_{2} \mathrm{Cl}_{2}$ ) with the $M N 15^{8}$ functional and a split basis set [LanL2DZ for Ti, 6-31+G(d)]. ${ }^{9,10,11}$ These structures were confirmed as maxima or minima using frequency calculations (zero imaginary frequencies for intermediates, one imaginary frequency for transition structures). Free energies in solution were derived from these optimized structures by means of a single-point calculation using the def2-QZVPP ${ }^{12}$ basis set with the IEF-PCM and SMD solvation models (solvent $=\mathrm{CH}_{2} \mathrm{Cl}_{2}$ ). These single-point calculations were used to correct the MN15/LanL2DZ for Ti, 6-31+G(d) energy. The free energy corrections were calculated using Truhlar's quasi-harmonic approximation ${ }^{13}$ as implemented

4 Schrödinger Release 2020-4, Maestro, Schrödinger, LLC, New York, NY, 2020.

5 Frisch, M. J.; Trucks, G. W.; Schlegel, H. B.; Scuseria, G. E.; Robb, M. A.; Cheeseman, J. R.; Scalmani, G.; Barone, V.; Petersson, G. A.; Nakatsuji, H.; Li, X.; Caricato, M.; Marenich, A. V.; Bloino, J.; Janesko, B. G.; Gomperts, R.; Mennucci, B.; Hratchian, H. P.; Ortiz, J. V.; Izmaylov, A. F.; Sonnenberg, J. L.; Williams-Young, D.; Ding, F.; Lipparini, F.; Egidi, F.; Goings, J.; Peng, B.; Petrone, A.; Henderson, T.; Ranasinghe, D.; Zakrzewski, V. G.; Gao, J.; Rega, N.; Zheng, G.; Liang, W.; Hada, M.; Ehara, M.; Toyota, K.; Fukuda, R.; Hasegawa, J.; Ishida, M.; Nakajima, T.; Honda, Y.; Kitao, O.; Nakai, H.; Vreven, T.; Throssell, K.; Montgomery, J. A., Jr.; Peralta, J. E.; Ogliaro, F.; Bearpark, M. J.; Heyd, J. J.; Brothers, E. N.; Kudin, K. N.; Staroverov, V. N.; Keith, T. A.; Kobayashi, R.; Normand, J.; Raghavachari, K.; Rendell, A. P.; Burant, J. C.; lyengar, S. S.; Tomasi, J.; Cossi, M.; Millam, J. M.; Klene, M.; Adamo, C.; Cammi, R.; Ochterski, J. W.; Martin, R. L.; Morokuma, K.; Farkas, O.; Foresman, J. B.; Fox, D. J. Gaussian 16, Revision C.01, Gaussian, Inc., Wallingford CT, 2016.

Cancès, E.; Mennucci, B.; Tomasi, J. J. Chem. Phys. 1997, 107, 3032-3041.

7 Marenich, A. V.; Cramer, C. J.; Truhlar, D. G. J. Phys. Chem. B 2009, 113, 6378-6396.

8 Yu, H. S.; He, X.; Li, S. L.; Truhlar, D. G. Chem. Sci. 2016, 7, 5032-5051.

9 Hay, P. J.; Wadt, W. R. J. Chem. Phys. 1985, 82, 299-310.

10 a) Hehre, W. J.; Ditchfield, R.; Pople, J. A. J. Chem. Phys. 1972, 56, 2257-2261. b) Francl, M. M.; Pietro, W. J.; Hehre, W. J.; Binkley, J. S.; Gordon, M. S.; DeFrees, D. J.; Pople, J. A. J. Chem. Phys. 1982, 77, 3654-3665.

11 Yang, Y.; Weaver, M. N.; Merz, K. M., Jr. J. Phys. Chem. A 2009, 113, 9843-9851.

12 a) Weigend, F.; Ahlrichs, R. Phys. Chem. Chem. Phys. 2005, 7, 3297-3305. b) Weigend, F. Phys. Chem. Chem. Phys. 2006, 8, 1057-1065.

13 Ribeiro, R. F.; Marenich, A. V.; Cramer, C. J.; Truhlar, D. G. J. Phys. Chem. B 2011, 115, 14556-14562. 
within GoodVibes ${ }^{14}$ along with temperature and concentration corrections (273.15 K, $\left.1.0 \mathrm{M}\right)$. GoodVibes was also used for the Boltzmann averaging of starting conformers and for generating potential energy surfaces for Figures S1 and S3.

The following information is disclosed for each calculated structure:

- A three-dimensional view of the optimized structure prepared using CYLview20. ${ }^{15}$

- The "Sum of the electronic and thermal Free Energies" in Hartree (from the optimization calculation).

- The energy from the single-point calculation ("E $E_{S P C}$ ") in Hartree.

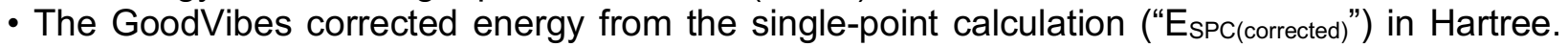

- The Cartesian coordinates found from the optimization calculation.

- Methods check for calculating aziridine opening via phenonium formation

Two functionals (MN15 and $\omega B$ 97X-D ${ }^{16}$ ), two split basis sets [LanL2DZ for Ti, 6-31+G(d) and def2-SVP ${ }^{9}$ for $\left.\mathrm{Ti}, 6-31+G(\mathrm{~d})\right]$ were used to evaluate the transformation of Int-1 to Int-2 via transition structure TS-1. The resulting $\Delta \mathrm{G}_{\text {rel }}$ values were compared to each other to check for consistency. Overall, the variable combinations of functionals and basis sets predicted similar $\mathrm{G}_{\mathrm{rel}}$ values for both TS-1 and Int-2. The consistency found between variations helped to reassure us that our choices of functional and basis set were suitable for our investigation.

14 GoodVibes, version 3.0.1, Luchini, G.; Alegre-Requena, J. V.; Funes-Ardoiz, I.; Paton, R. S. F1000Research 2020, 9, 291.

15 CYLview20; Legault, C. Y., Université de Sherbrooke, 2020 (http://www.cylview.org).

16 a) Becke, A. D. J. Chem. Phys. 1997, 107, 8554-8560. b) Chai, J.-D.; Head-Gordon, M. Phys. Chem. Chem. Phys. 2008, 10, 6615-6620. 
Table S2: Comparison of functionals and basis sets used for computing phenonium ion formation.

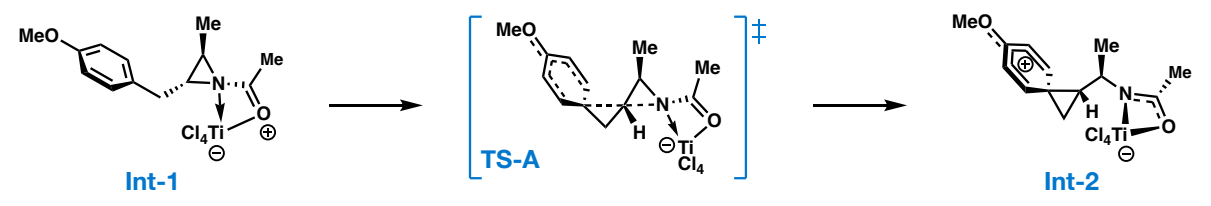

\begin{tabular}{|c|c|c|c|}
\hline & Int-1 & TS-A & Int-2 \\
\hline (R)MN15/LanL2DZ(Ti),6-31+G(d) & 0.00 & 10.36 & 0.21 \\
\hline (R)wB97X-D/LanL2DZ(Ti),6-31+G(d) & 0.00 & 11.22 & 0.44 \\
\hline (R)MN15/Def2-SVP(Ti),6-31+G(d) & 0.00 & 10.49 & 0.31 \\
\hline
\end{tabular}

\begin{tabular}{c|c|c|c|}
\cline { 2 - 4 }$(\mathrm{R}) M N 15 / L a n L 2 D Z(T i), 6-31+G(d)$ & 0.00 & 10.81 & 0.72 \\
\cline { 2 - 4 }$(\mathrm{R}) \omega B 97 X-D / L a n L 2 D Z(T i), 6-31+G(d)$ & 0.00 & 11.16 & 0.40 \\
\cline { 2 - 4 }$(\mathrm{R}) M N 15 / \operatorname{Def2-SVP(Ti),6-31+G(d)}$ & 0.00 & 10.45 & 0.34 \\
\cline { 2 - 4 } & &
\end{tabular}

all values are in $\mathrm{kcal} \mathrm{mol}^{-1}$; corrected for $0^{\circ} \mathrm{C} / 1.0 \mathrm{M}$

\section{Discussion}

The different aspects studied in this chemistry included the effects of the $N$-protecting group (NTs vs. NAc), the nature of aziridine coordination to $\mathrm{TiCl}_{4}$, and the relative configuration of the stereogenic aziridine $N$ center to the carbon backbone (when bidentate $N, O$-coordination was occurring). In all cases with bidentate $\mathrm{N}, \mathrm{O}$-coordination these conclusions could be drawn: 1 ) aziridine opening via phenonium ion formation was energetically viable (i.e. reasonable $G_{\text {rel }}$ for TS1), and 2) formation of the rearranged product (Int2 $->$ TS2-major -> Int3-major) was favored over formation of the retentive opening product (Int2 -> TS2-minor -> Int3-minor). 
A)
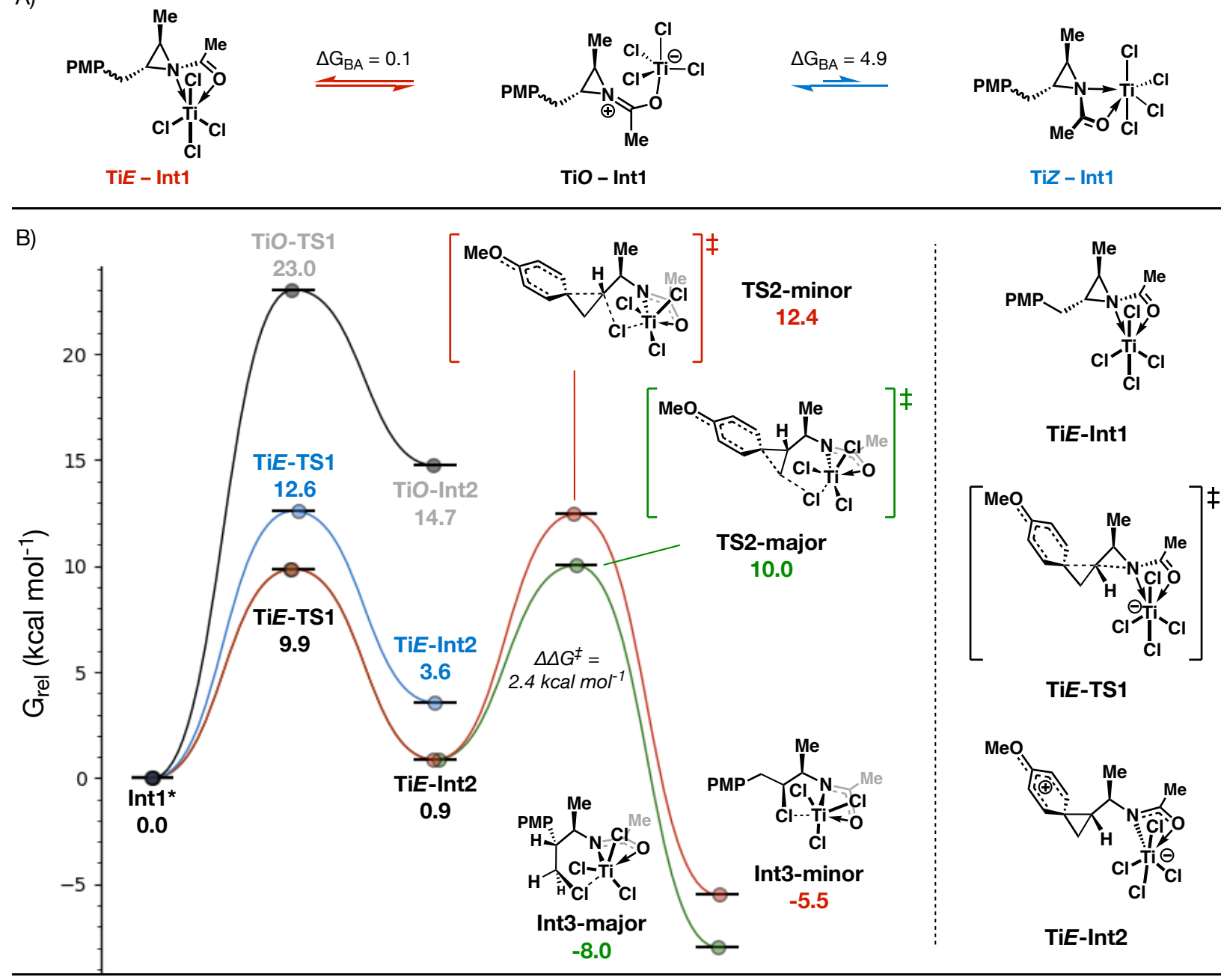

reaction coordinate

Figure S1: A) Computed equilibrium of different Ti-coordination species. $\Delta G_{B A}$ represents the difference in energy for each Boltzmann averaged species. B) Potential energy surface for phenonium ion formation for all three species along with phenonium ion opening for the TiE pathway.

Our investigation began with probing the nature of $\mathrm{TiCl}_{4}$ coordination to the $\mathrm{N}$-Ac aziridine. We found that the Boltzmann averaged starting structures showed an energetic preference for monodentate O-ligation to the acyl group (TiO-Int1) compared to bidentate $\mathrm{N}, \mathrm{O}$-ligation to form a titanacycle with an octahedral geometry (TiE-Int1 and TiZ-Int1, Figure S1a). However, only a small energy barrier between TiO-Int1 and TiE-Int1 was found, so we presume that these species would exist in equilibrium. Though the bidentate complexes were slightly higher in energy they had substantially lower $\mathrm{G}_{\text {rel }}$ values for TS1 and Int2. $\Delta \mathrm{G}^{\ddagger}$ for the monodentate complex was $23.0 \mathrm{kcal} \mathrm{mol}^{-1}$, whereas the bidentate Ti complexes were much lower at $\left(9.9 \mathrm{kcal} \mathrm{mol}^{-1}\right.$ for TiETS1 and 12.6 for TiZ-TS1, Figure S1b). From this finding we concluded that phenonium formation would almost entirely occur through the bidentate Ti complexes. This was used as the basis for the rest of this study. 


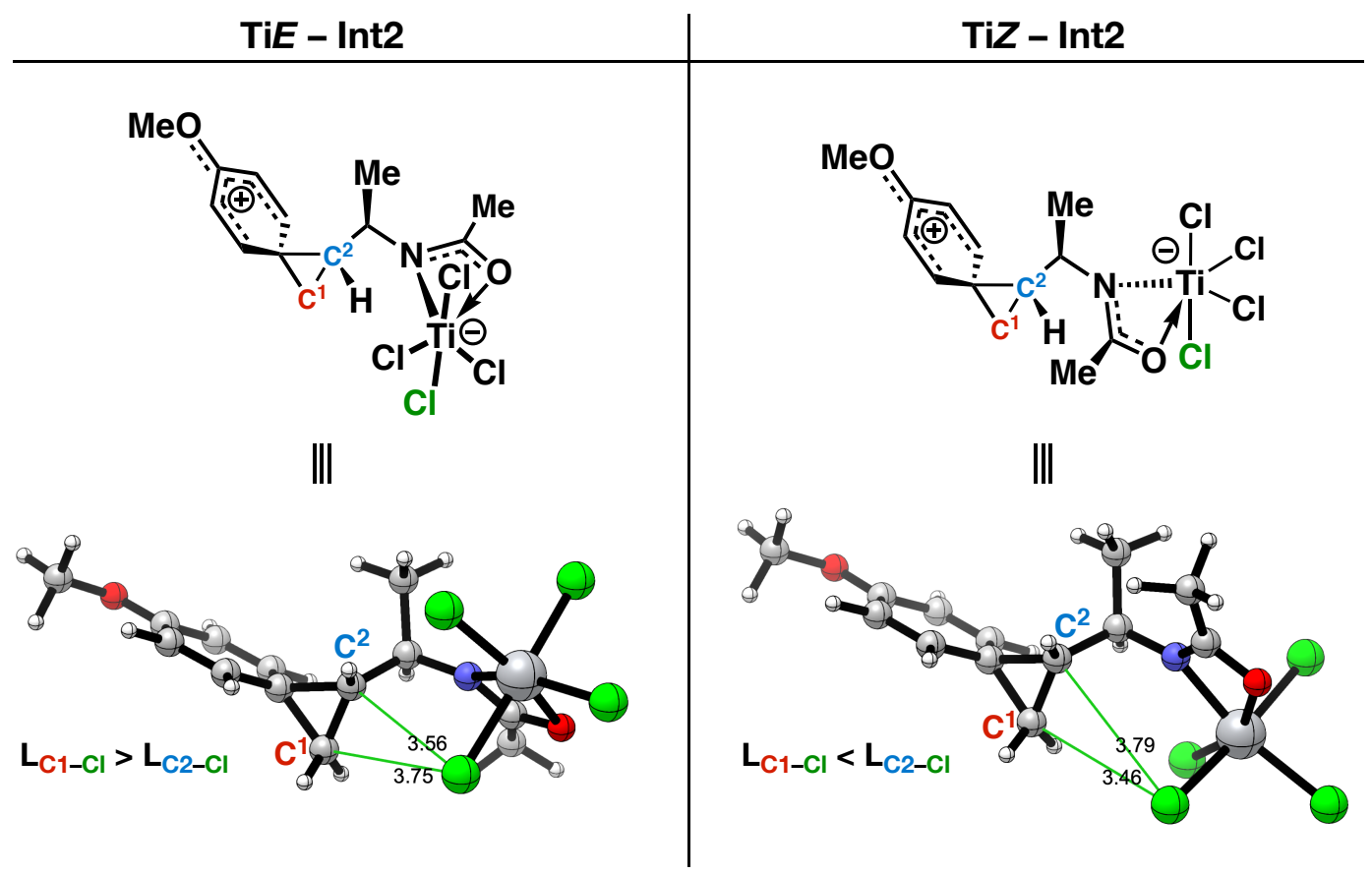

Figure S2: Comparison of isomeric phenonium ions TiE-Int2 and TiZ-Int2.

Two stereoisomeric forms of the bidentate $N$-Ac aziridine were found (i.e., TiE-Int1 and TiZ-Int1). These species differed in the configuration of the stereogenic, pseudo-tetrahedral $N$ center (with Ti either $E$ or $Z$ to the PMB group). Though the reaction pathways forming Int3-major for each stereoisomer were paralleled (same trend, differed slightly energetically), a TS2-minor structure could not be successfully optimized from TiZ-Int2. Inspection of the geometry of TiZInt2 showed that the $\mathrm{C} 2-\mathrm{Cl}$ distance $(3.79 \AA)$ was longer than the $\mathrm{C} 1-\mathrm{Cl}$ distance $(3.46 \AA)$ to the attacking chloride (Figure S2). C1, in closer proximity to the chloride, obstructed the trajectory for the formation of the retentive-opening product (Int3-minor). In each attempt to optimize a TiZTS2-minor structure $\mathrm{C} 1$ was found to accept the chloride before it could reach $\mathrm{C} 2$. Contrastingly, TiE-Int2 had a longer $\mathrm{C} 1-\mathrm{Cl}$ distance $(3.75 \AA)$ than $\mathrm{C} 2-\mathrm{Cl}(3.56 \AA)$, which allowed for the chloride to approach $\mathrm{C} 2$ without being thwarted by $\mathrm{C} 1$. Whereas the two aziridines (i.e. Int1) were stereoisomeric, once phenonium ion formation occurred the two species became conformational isomers since the newly $\mathrm{sp}^{2}$-hybridized $N$ center could then freely rotate. In theory, TiZ-Int2 could undergo a $180^{\circ}$ dihedral rotation of the $\mathrm{C}\left(\mathrm{sp}^{3}\right)-\mathrm{N}$ bond to effectively form TiE-Int2. An energy barrier for rotation between the Int2 structures was not calculated. 


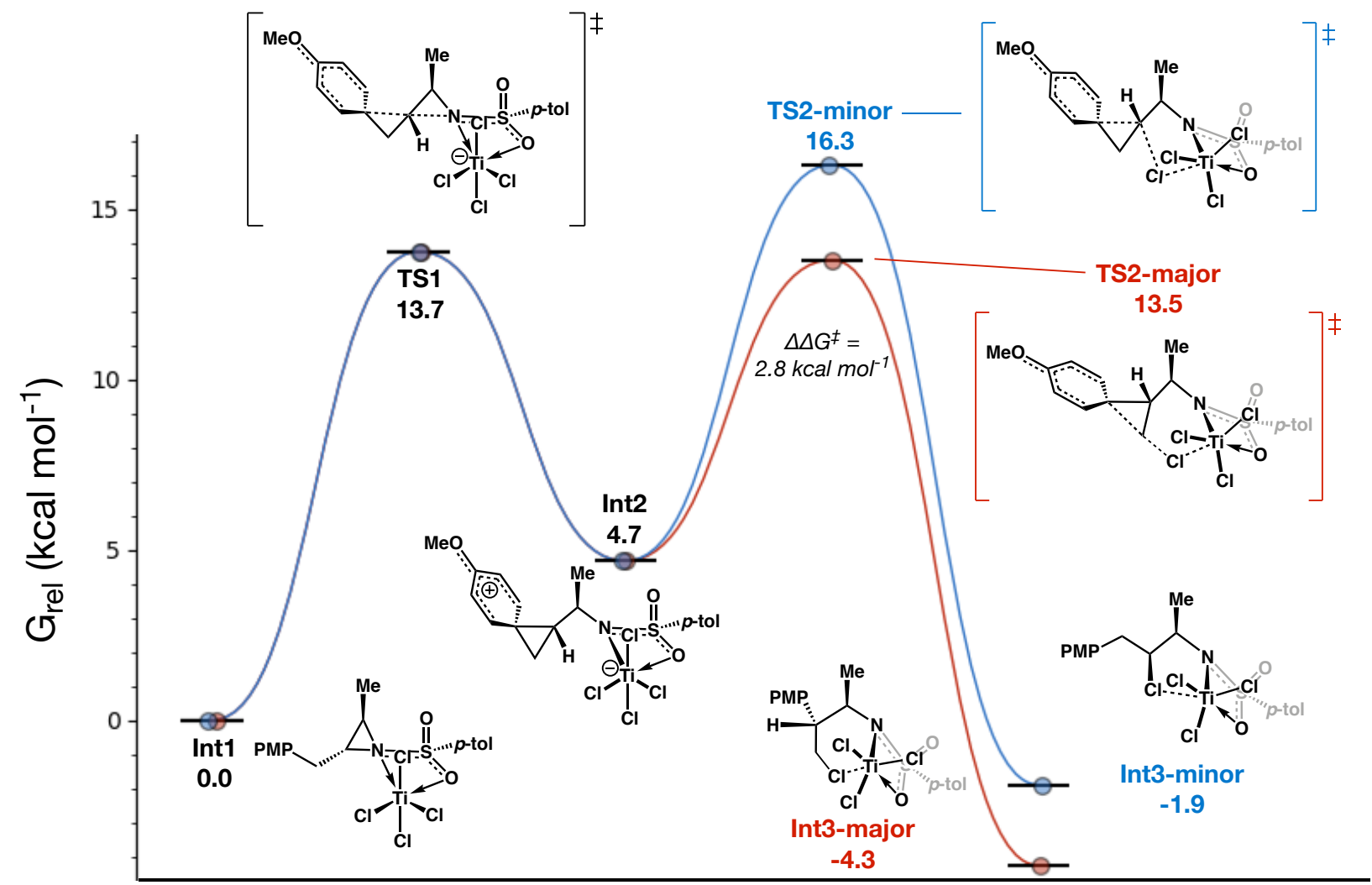

reaction coordinate

Figure S3: Potential energy surface for $\mathrm{N}$-Ts aziridine reaction pathways.

Like the $N$-Ac aziridines the $N$-Ts aziridine titanacycles demonstrated bidentate $\mathrm{N}, \mathrm{O}$-coordination to $\mathrm{TiCl}_{4}$. Additionally, with this system a further consideration for the relative orientation of the tolyl group was introduced (E-syn, E-anti, Z-syn, Z-anti; E/Z with respect to $\mathrm{Ti}$ and the PMB group, syn/anti with respect to the tolyl and PMB group). Again, geometries for TS2minor were only found for the E-syn and E-anti configurations due to the same geometric constraints as stated above. Minor fluctuations in energies were found between the four sets of conformational isomers, but they all showed similar energetics across the calculated structures. The $E$-anti pathway is graphed in the potential energy surface shown above (Figure S3). The $\mathrm{G}_{\mathrm{rel}}$ values were found to be higher than the $N$-Ac pathway, but they were still reasonable values that mirrored the same trend shown for the $\mathrm{N}$-Ac aziridine. 


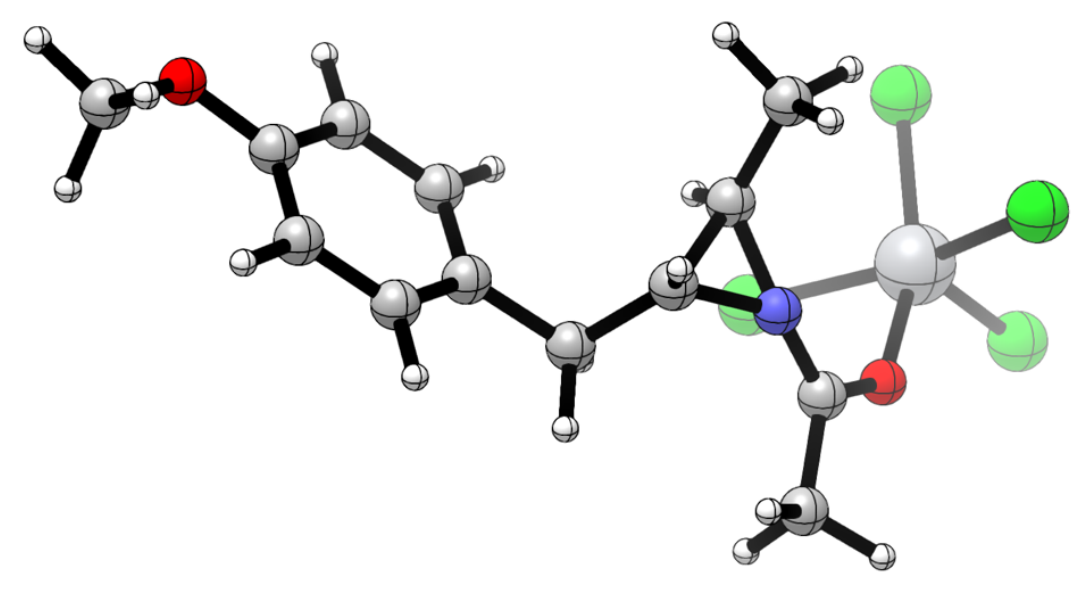

Sum of electronic and thermal free energies: -2608.223039 a.u.

$E_{\text {sPC }}\left[\left(I E F-P C M: \mathrm{CH}_{2} \mathrm{Cl}_{2}, \mathrm{SMD}\right) / \mathrm{MN15} /\right.$ def2-QZVPP] $=-3401.242098$ a.u.

$\mathrm{E}_{\mathrm{SPC}(\text { corrected) }}\left[\left(\mathrm{IEF}-\mathrm{PCM}: \mathrm{CH}_{2} \mathrm{Cl}_{2}, \mathrm{SMD}\right) / \mathrm{MN15} /\right.$ def2-QZVPP, $\left.0{ }^{\circ} \mathrm{C}, 1.0 \mathrm{M}\right]=-3400.998145$ a.u.

Number of imaginary frequencies: 0

\begin{tabular}{|c|c|c|c|c|c|}
\hline \multirow{2}{*}{$\begin{array}{l}\text { Center } \\
\text { Number }\end{array}$} & \multirow{2}{*}{$\begin{array}{l}\text { Atomic } \\
\text { Number }\end{array}$} & \multirow{2}{*}{$\begin{array}{c}\text { Atomic } \\
\text { Type }\end{array}$} & \multicolumn{3}{|c|}{ Coordinates (Ångstroms) } \\
\hline & & & $X$ & $\mathrm{Y}$ & Z \\
\hline 1 & 6 & 0 & 4.50600 & -1.42250 & -0.39890 \\
\hline 2 & 6 & 0 & 3.29590 & -0.74950 & -0.52450 \\
\hline 3 & 6 & 0 & 3.23040 & 0.65190 & -0.42760 \\
\hline 4 & 6 & 0 & 4.41780 & 1.35160 & -0.20160 \\
\hline 5 & 6 & 0 & 5.64690 & 0.69160 & -0.07330 \\
\hline 6 & 6 & 0 & 5.69130 & -0.70400 & -0.17070 \\
\hline 7 & 8 & 0 & 6.82950 & -1.43980 & -0.06390 \\
\hline 8 & 6 & 0 & 8.05190 & -0.75470 & 0.15530 \\
\hline 9 & 6 & 0 & 1.90190 & 1.36680 & -0.52800 \\
\hline 10 & 6 & 0 & 1.13100 & 1.25510 & 0.77320 \\
\hline 11 & 6 & 0 & 0.23230 & 0.06350 & 1.02380 \\
\hline 12 & 6 & 0 & 0.05140 & -0.47860 & 2.41760 \\
\hline 13 & 6 & 0 & -1.19580 & 1.98010 & -0.04060 \\
\hline 14 & 6 & 0 & -0.91760 & 3.35020 & -0.56080 \\
\hline 15 & 8 & 0 & -2.31750 & 1.43410 & -0.31440 \\
\hline 16 & 1 & 0 & 4.55810 & -2.50610 & -0.48090 \\
\hline 17 & 1 & 0 & 2.38420 & -1.31710 & -0.71220 \\
\hline 18 & 1 & 0 & 4.39220 & 2.43840 & -0.12750 \\
\hline 19 & 1 & 0 & 6.54940 & 1.27170 & 0.09700 \\
\hline
\end{tabular}




$\begin{array}{rrrrrr}20 & 1 & 0 & 8.02880 & -0.19550 & 1.10020 \\ 21 & 1 & 0 & 8.82870 & -1.52040 & 0.20710 \\ 22 & 1 & 0 & 8.27360 & -0.06690 & -0.67150 \\ 23 & 1 & 0 & 2.05920 & 2.42580 & -0.76950 \\ 24 & 1 & 0 & 1.28410 & 0.92860 & -1.32720 \\ 25 & 1 & 0 & 1.58920 & 1.70340 & 1.65630 \\ 26 & 1 & 0 & 0.16190 & -0.65320 & 0.20240 \\ 27 & 1 & 0 & 0.88720 & -1.14910 & 2.64980 \\ 28 & 1 & 0 & 0.03960 & 0.33800 & 3.14900 \\ 29 & 1 & 0 & -0.88110 & -1.04600 & 2.50520 \\ 30 & 1 & -0.69570 & 3.26850 & -1.63320 \\ 31 & 1 & 0 & -1.81140 & 3.97090 & -0.44610 \\ 32 & 1 & -0.06730 & 3.80620 & -0.04830 \\ 33 & 7 & 0 & -0.30420 & 1.37470 & 0.71560 \\ 34 & 22 & 0 & -3.09840 & -0.35040 & -0.17590 \\ 35 & 17 & 0 & -3.06090 & -2.51850 & 0.41360 \\ 36 & 17 & 0 & -3.52350 & 0.28000 & 1.96490 \\ 37 & 17 & -5.03810 & -0.10520 & -1.10500 \\ 38 & 17 & 0 & -1.76830 & -0.78420 & -1.98680 \\ ---------------------------------------------------------------------------------------------\end{array}$




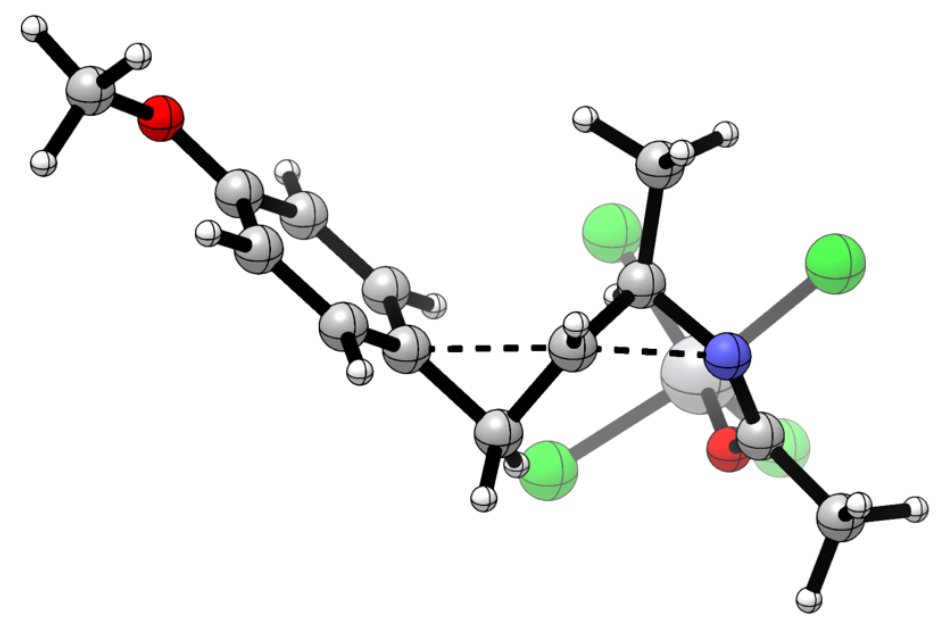

Sum of electronic and thermal free energies: -2608.187235 a.u.

$\mathrm{E}_{\mathrm{SPC}}\left[\left(\mathrm{IEF}-\mathrm{PCM}: \mathrm{CH}_{2} \mathrm{Cl}_{2}, \mathrm{SMD}\right) / \mathrm{MN} 15 /\right.$ def2-QZVPP] $=-3401.202831$ a.u.

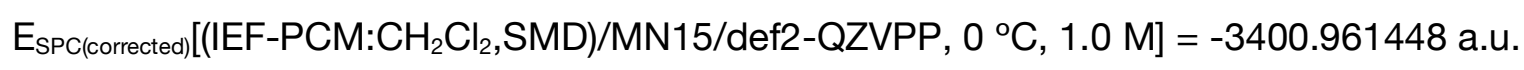

Number of imaginary frequencies: 1

\begin{tabular}{|c|c|c|c|c|c|}
\hline \multirow{2}{*}{$\begin{array}{l}\text { Center } \\
\text { Number }\end{array}$} & \multirow{2}{*}{$\begin{array}{l}\text { Atomic } \\
\text { Number }\end{array}$} & \multirow{2}{*}{$\begin{array}{l}\text { Atomic } \\
\text { Type }\end{array}$} & \multicolumn{3}{|c|}{ Coordinates (Ångstroms) } \\
\hline & & & $\mathrm{X}$ & $\mathrm{Y}$ & Z \\
\hline 1 & 6 & 0 & 3.43840 & -1.32050 & -0.02920 \\
\hline 2 & 6 & 0 & 2.33730 & -0.61050 & -0.47300 \\
\hline 3 & 6 & 0 & 2.47920 & 0.72250 & -0.92170 \\
\hline 4 & 6 & 0 & 3.74560 & 1.32820 & -0.86260 \\
\hline 5 & 6 & 0 & 4.86040 & 0.62390 & -0.41440 \\
\hline 6 & 6 & 0 & 4.70800 & -0.70870 & 0.00390 \\
\hline 7 & 8 & 0 & 5.71970 & -1.47480 & 0.45390 \\
\hline 8 & 6 & 0 & 7.02760 & -0.91740 & 0.51840 \\
\hline 9 & 6 & 0 & 1.26520 & 1.51150 & -1.41580 \\
\hline 10 & 6 & 0 & 1.06210 & 1.98640 & -0.04920 \\
\hline 11 & 6 & 0 & 0.14620 & 1.33400 & 0.91280 \\
\hline 12 & 6 & 0 & 0.56510 & 1.30610 & 2.36590 \\
\hline 13 & 6 & 0 & -1.74930 & 2.24480 & -0.24030 \\
\hline 14 & 6 & 0 & -2.57560 & 3.39350 & -0.71350 \\
\hline 15 & 8 & 0 & -2.13810 & 1.06470 & -0.65760 \\
\hline 16 & 1 & 0 & 3.34690 & -2.35270 & 0.29930 \\
\hline 17 & 1 & 0 & 1.35850 & -1.08920 & -0.50100 \\
\hline 18 & 1 & 0 & 3.85790 & 2.36150 & -1.18780 \\
\hline 19 & 1 & 0 & 5.83050 & 1.11060 & -0.39310 \\
\hline
\end{tabular}




\begin{tabular}{|c|c|c|c|c|c|}
\hline 20 & 1 & 0 & 7.05150 & -0.05410 & 1.19480 \\
\hline 21 & 1 & 0 & 7.67370 & -1.70530 & 0.90940 \\
\hline 22 & 1 & 0 & 7.37490 & -0.61950 & -0.47860 \\
\hline 23 & 1 & 0 & 1.53610 & 2.28690 & -2.13360 \\
\hline 24 & 1 & 0 & 0.47180 & 0.85500 & -1.78380 \\
\hline 25 & 1 & 0 & 1.63860 & 2.83790 & 0.31120 \\
\hline 26 & 1 & 0 & -0.18090 & 0.34300 & 0.56320 \\
\hline 27 & 1 & 0 & 1.36890 & 0.57250 & 2.50310 \\
\hline 28 & 1 & 0 & 0.92430 & 2.29450 & 2.67600 \\
\hline 29 & 1 & 0 & -0.28550 & 1.02200 & 2.99480 \\
\hline 30 & 1 & 0 & -2.14140 & 4.34040 & -0.38630 \\
\hline 31 & 1 & 0 & -2.65200 & 3.36730 & -1.80670 \\
\hline 32 & 1 & 0 & -3.59040 & 3.29060 & -0.30790 \\
\hline 33 & 7 & 0 & -0.73200 & 2.44070 & 0.56340 \\
\hline 34 & 22 & 0 & -2.38440 & -0.70910 & -0.08730 \\
\hline 35 & 17 & 0 & -1.31330 & -2.34150 & 1.04950 \\
\hline 36 & 17 & 0 & -2.98980 & 0.22850 & 1.92310 \\
\hline 37 & 17 & 0 & -4.36000 & -1.41720 & -0.68800 \\
\hline 38 & 17 & 0 & -1.24970 & -1.31850 & -1.98780 \\
\hline
\end{tabular}




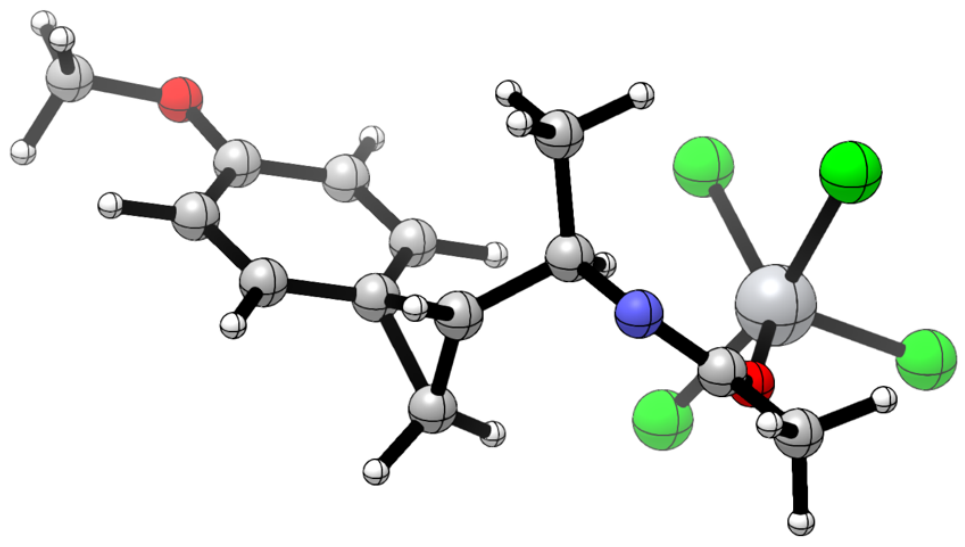

Sum of electronic and thermal free energies: -2608.201576 a.u.

$E_{\text {SPC }}\left[\left(I E F-P C M: \mathrm{CH}_{2} \mathrm{Cl}_{2}, \mathrm{SMD}\right) / \mathrm{MN15} /\right.$ def2-QZVPP] $=-3401.218325$ a.u.

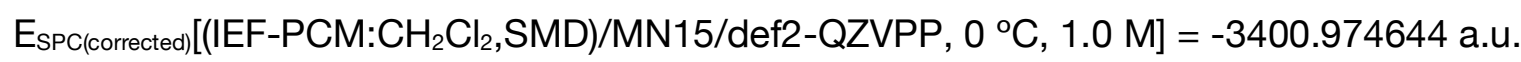

Number of imaginary frequencies: 0

\begin{tabular}{|c|c|c|c|c|c|}
\hline \multirow{2}{*}{$\begin{array}{l}\text { Center } \\
\text { Number }\end{array}$} & \multirow{2}{*}{$\begin{array}{l}\text { Atomic } \\
\text { Number }\end{array}$} & \multirow{2}{*}{$\begin{array}{c}\text { Atomic } \\
\text { Type }\end{array}$} & \multicolumn{3}{|c|}{ Coordinates (Ångstroms) } \\
\hline & & & $\mathrm{X}$ & Y & $\mathrm{Z}$ \\
\hline 1 & 6 & 0 & 3.30730 & -1.24870 & 0.03770 \\
\hline 2 & 6 & 0 & 2.23830 & -0.48470 & -0.33460 \\
\hline 3 & 6 & 0 & 2.41150 & 0.90060 & -0.69610 \\
\hline 4 & 6 & 0 & 3.74450 & 1.43650 & -0.67700 \\
\hline 5 & 6 & 0 & 4.82360 & 0.68050 & -0.29860 \\
\hline 6 & 6 & 0 & 4.60920 & -0.67450 & 0.07120 \\
\hline 7 & 8 & 0 & 5.57620 & -1.48130 & 0.45860 \\
\hline 8 & 6 & 0 & 6.92990 & -1.01160 & 0.53770 \\
\hline 9 & 6 & 0 & 1.38340 & 1.54070 & -1.70610 \\
\hline 10 & 6 & 0 & 1.27640 & 1.96760 & -0.32900 \\
\hline 11 & 6 & 0 & 0.13730 & 1.54600 & 0.58730 \\
\hline 12 & 6 & 0 & 0.58360 & 1.48840 & 2.04350 \\
\hline 13 & 6 & 0 & -2.01040 & 2.24850 & -0.14490 \\
\hline 14 & 6 & 0 & -3.11140 & 3.23930 & -0.35130 \\
\hline 15 & 8 & 0 & -2.31940 & 1.01420 & -0.59950 \\
\hline 16 & 1 & 0 & 3.19570 & -2.29450 & 0.30940 \\
\hline 17 & 1 & 0 & 1.24770 & -0.93350 & -0.37700 \\
\hline 18 & 1 & 0 & 3.88260 & 2.47770 & -0.96420 \\
\hline 19 & 1 & 0 & 5.81920 & 1.11080 & -0.28360 \\
\hline 20 & 1 & 0 & 7.00080 & -0.18030 & 1.24630 \\
\hline
\end{tabular}




$\begin{array}{rrrrrr}21 & 1 & 0 & 7.51370 & -1.85910 & 0.89670 \\ 22 & 1 & 0 & 7.28250 & -0.70630 & -0.45250 \\ 23 & 1 & 0 & 1.84150 & 2.19300 & -2.44430 \\ 24 & 1 & 0 & 0.66170 & 0.81590 & -2.08140 \\ 25 & 1 & 0 & 1.71760 & 2.92720 & -0.05980 \\ 26 & 1 & 0 & -0.20580 & 0.54220 & 0.27820 \\ 27 & 1 & 0 & 1.35640 & 0.71960 & 2.17550 \\ 28 & 1 & 0 & 0.99290 & 2.45960 & 2.34980 \\ 29 & 1 & 0 & -0.26600 & 1.24410 & 2.69050 \\ 30 & 1 & -3.38030 & 3.28290 & -1.41320 \\ 31 & 1 & 0 & -3.99950 & 2.91340 & 0.20530 \\ 32 & 1 & 0 & -2.80220 & 4.22840 & -0.00480 \\ 33 & 7 & 0 & -2.89800 & 2.54820 & 0.41130 \\ 34 & 22 & 0 & -0.97110 & -2.26000 & 0.97540 \\ 35 & 17 & 0 & -2.88660 & 0.04450 & 2.02140 \\ 36 & 17 & 0 & -4.25340 & -1.62640 & -0.59710 \\ 37 & 17 & 0 & -1.22200 & -1.26720 & -2.06500 \\ 38 & 17 & 0.0800\end{array}$




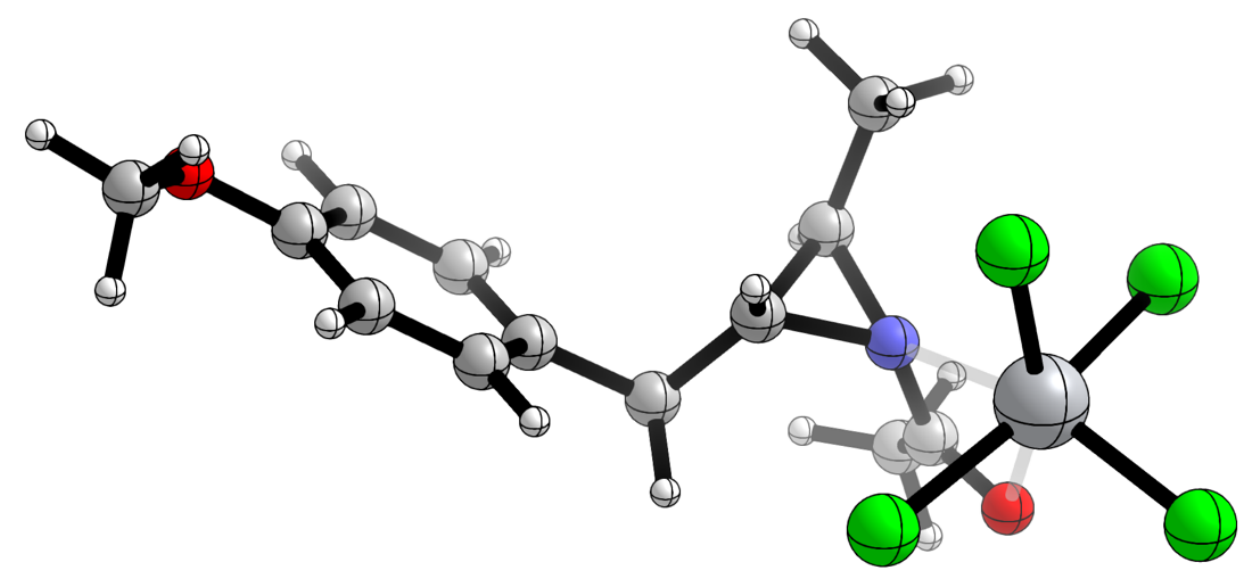

Sum of electronic and thermal free energies: -2608.218358 a.u.

$E_{\text {sPC }}\left[\left(I E F-P C M: \mathrm{CH}_{2} \mathrm{Cl}_{2}, \mathrm{SMD}\right) / \mathrm{MN15} /\right.$ def2-QZVPP] $=-3401.236240$ a.u.

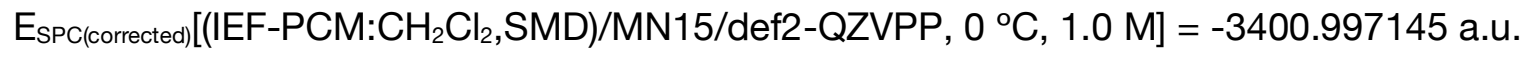

Number of imaginary frequencies: 0

\begin{tabular}{|c|c|c|c|c|c|}
\hline \multirow{2}{*}{$\begin{array}{l}\text { Center } \\
\text { Number }\end{array}$} & \multirow{2}{*}{$\begin{array}{l}\text { Atomic } \\
\text { Number }\end{array}$} & \multirow{2}{*}{$\begin{array}{l}\text { Atomic } \\
\text { Type }\end{array}$} & \multicolumn{3}{|c|}{ Coordinates (Ångstroms) } \\
\hline & & & $\mathrm{X}$ & $\mathrm{Y}$ & $\mathrm{Z}$ \\
\hline 1 & 6 & 0 & -4.56700 & -1.12030 & -0.39010 \\
\hline 2 & 6 & 0 & -3.26970 & -0.76330 & -0.77390 \\
\hline 3 & 6 & 0 & -2.82450 & 0.56010 & -0.70360 \\
\hline 4 & 6 & 0 & -3.71300 & 1.53630 & -0.22580 \\
\hline 5 & 6 & 0 & -5.00720 & 1.20050 & 0.15980 \\
\hline 6 & 6 & 0 & -5.44180 & -0.13190 & 0.08020 \\
\hline 7 & 8 & 0 & -6.72030 & -0.37260 & 0.47370 \\
\hline 8 & 6 & 0 & -7.20340 & -1.70500 & 0.40790 \\
\hline 9 & 6 & 0 & -1.40550 & 0.91800 & -1.08860 \\
\hline 10 & 6 & 0 & -0.49750 & 0.64740 & 0.09040 \\
\hline 11 & 6 & 0 & 0.04300 & 1.68560 & 0.98630 \\
\hline 12 & 6 & 0 & 0.27290 & 1.47670 & 2.45610 \\
\hline 13 & 6 & 0 & 1.62370 & 1.66720 & -1.00820 \\
\hline 14 & 6 & 0 & 1.37080 & 3.06520 & -1.42680 \\
\hline 15 & 8 & 0 & 2.56620 & 0.99550 & -1.46160 \\
\hline 16 & 1 & 0 & -4.88040 & -2.15770 & -0.46450 \\
\hline 17 & 1 & 0 & -2.59340 & -1.53590 & -1.14130 \\
\hline 18 & 1 & 0 & -3.38620 & 2.57410 & -0.16290 \\
\hline 19 & 1 & 0 & -5.69960 & 1.95640 & 0.52400 \\
\hline
\end{tabular}




$\begin{array}{rrrrrr}20 & 1 & 0 & -8.23560 & -1.67520 & 0.76290 \\ 21 & 1 & 0 & -7.18400 & -2.08250 & -0.62310 \\ 22 & 1 & 0 & -6.61590 & -2.37150 & 1.05330 \\ 23 & 1 & 0 & -1.07300 & 0.30490 & -1.93660 \\ 24 & 1 & 0 & -1.35210 & 1.97150 & -1.38320 \\ 25 & 1 & 0 & -0.60420 & -0.35160 & 0.51950 \\ 26 & 1 & 0 & -0.15710 & 2.71320 & 0.67800 \\ 27 & 1 & 0 & -0.63480 & 1.76980 & 2.99680 \\ 28 & 1 & 0 & 0.48680 & 0.42650 & 2.67830 \\ 29 & 1 & 0 & 1.10400 & 2.09500 & 2.81120 \\ 30 & 1 & 0 & 1.79120 & 3.71350 & -0.64240 \\ 31 & 1 & 0 & 1.89330 & 3.25860 & -2.36590 \\ 32 & 1 & 0 & 0.30640 & 3.29800 & -1.51540 \\ 33 & 22 & 0 & 2.58410 & -0.57900 & 0.02510 \\ 34 & 17 & 0 & 3.64960 & 0.96590 & 1.32810 \\ 35 & 17 & 0 & 1.88490 & -1.82020 & 1.72270 \\ 36 & 17 & 0 & 1.20760 & -1.61670 & -1.48110 \\ 37 & 17 & 0 & 4.44160 & -1.48770 & -0.75200 \\ 38 & 7 & 0 & 0.97290 & 0.98950 & 0.04000 \\ ----------------------------------------------------------------------------------------------------\end{array}$




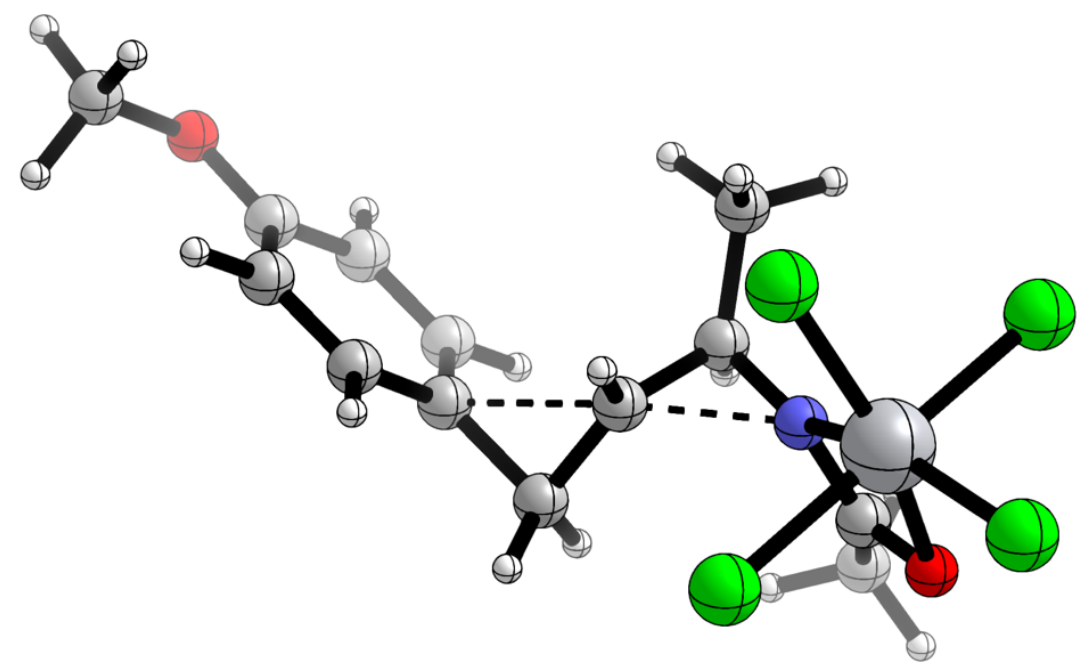

Sum of electronic and thermal free energies: -2608.201855 a.u.

$E_{S P C}\left[\left(I E F-P C M: \mathrm{CH}_{2} \mathrm{Cl}_{2}, \mathrm{SMD}\right) / \mathrm{MN15} /\right.$ def2-QZVPP] $=-3401.218092$ a.u.

$E_{S P C(\text { corrected) }}\left[\left(I E F-P C M: \mathrm{CH}_{2} \mathrm{Cl}_{2}, \mathrm{SMD}\right) / \mathrm{MN} 15 /\right.$ def2-QZVPP, $\left.0{ }^{\circ} \mathrm{C}, 1.0 \mathrm{M}\right]=-3400.980975$ a.u.

Number of imaginary frequencies: 1

\begin{tabular}{|c|c|c|c|c|c|}
\hline \multirow{2}{*}{$\begin{array}{l}\text { Center } \\
\text { Number }\end{array}$} & \multirow{2}{*}{$\begin{array}{l}\text { Atomic } \\
\text { Number }\end{array}$} & \multirow{2}{*}{$\begin{array}{l}\text { Atomic } \\
\text { Type }\end{array}$} & \multicolumn{3}{|c|}{ Coordinates (Ångstroms) } \\
\hline & & & X & Y & Z \\
\hline 1 & 6 & 0 & -4.57670 & -1.05340 & -0.63150 \\
\hline 2 & 6 & 0 & -3.33680 & -0.71230 & -1.16680 \\
\hline 3 & 6 & 0 & -2.78790 & 0.56460 & -0.96540 \\
\hline 4 & 6 & 0 & -3.48580 & 1.48990 & -0.15920 \\
\hline 5 & 6 & 0 & -4.71870 & 1.16240 & 0.37770 \\
\hline 6 & 6 & 0 & -5.27360 & -0.11200 & 0.14410 \\
\hline 7 & 8 & 0 & -6.47640 & -0.34400 & 0.70470 \\
\hline 8 & 6 & 0 & -7.09510 & -1.60960 & 0.50090 \\
\hline 9 & 6 & 0 & -1.42440 & 0.92750 & -1.55910 \\
\hline 10 & 6 & 0 & -0.78740 & 0.38610 & -0.36200 \\
\hline 11 & 6 & 0 & -0.33420 & 1.18520 & 0.79320 \\
\hline 12 & 6 & 0 & -0.52460 & 0.63250 & 2.18790 \\
\hline 13 & 6 & 0 & 1.72680 & 1.91020 & -0.38050 \\
\hline 14 & 6 & 0 & 1.34700 & 3.33210 & -0.59750 \\
\hline 15 & 8 & 0 & 2.87240 & 1.46380 & -0.72270 \\
\hline 16 & 1 & 0 & -4.98700 & -2.04150 & -0.81550 \\
\hline 17 & 1 & 0 & -2.79030 & -1.44000 & -1.76510 \\
\hline 18 & 1 & 0 & -3.05690 & 2.47470 & 0.02140 \\
\hline 19 & 1 & 0 & -5.27680 & 1.87200 & 0.98350 \\
\hline
\end{tabular}




$\begin{array}{rrrrrr}20 & 1 & 0 & -8.04750 & -1.57030 & 1.03210 \\ 21 & 1 & 0 & -7.27710 & -1.78810 & -0.56620 \\ 22 & 1 & 0 & -6.47800 & -2.41750 & 0.91310 \\ 23 & 1 & 0 & -1.20750 & 0.37340 & -2.47430 \\ 24 & 1 & 0 & -1.30950 & 2.00650 & -1.68250 \\ 25 & 1 & 0 & -0.64900 & -0.69470 & -0.29250 \\ 26 & 1 & 0 & -0.62650 & 2.23850 & 0.71370 \\ 27 & 1 & 0 & -1.58030 & 0.72090 & 2.47130 \\ 28 & 1 & 0 & -0.22970 & -0.42110 & 2.23570 \\ 29 & 1 & 0 & 0.08310 & 1.20340 & 2.89820 \\ 30 & 1 & 0 & 1.18250 & 3.82000 & 0.37190 \\ 31 & 1 & 0 & 2.15060 & 3.84270 & -1.13240 \\ 32 & 1 & 0 & 0.41530 & 3.40120 & -1.17100 \\ 33 & 22 & 0 & 2.62160 & -0.44790 & 0.03330 \\ 34 & 17 & 0 & 3.27130 & 0.36790 & 2.08160 \\ 35 & 17 & 0 & 1.52480 & -2.20520 & 0.92370 \\ 36 & 17 & 0 & 1.78010 & -0.92830 & -2.08810 \\ 37 & 17 & 0 & 4.68840 & -1.11880 & -0.50410 \\ 38 & 7 & 0 & 0.99610 & 0.96140 & 0.22230 \\ ---------------------------------------------------------------------------------------------------\end{array}$




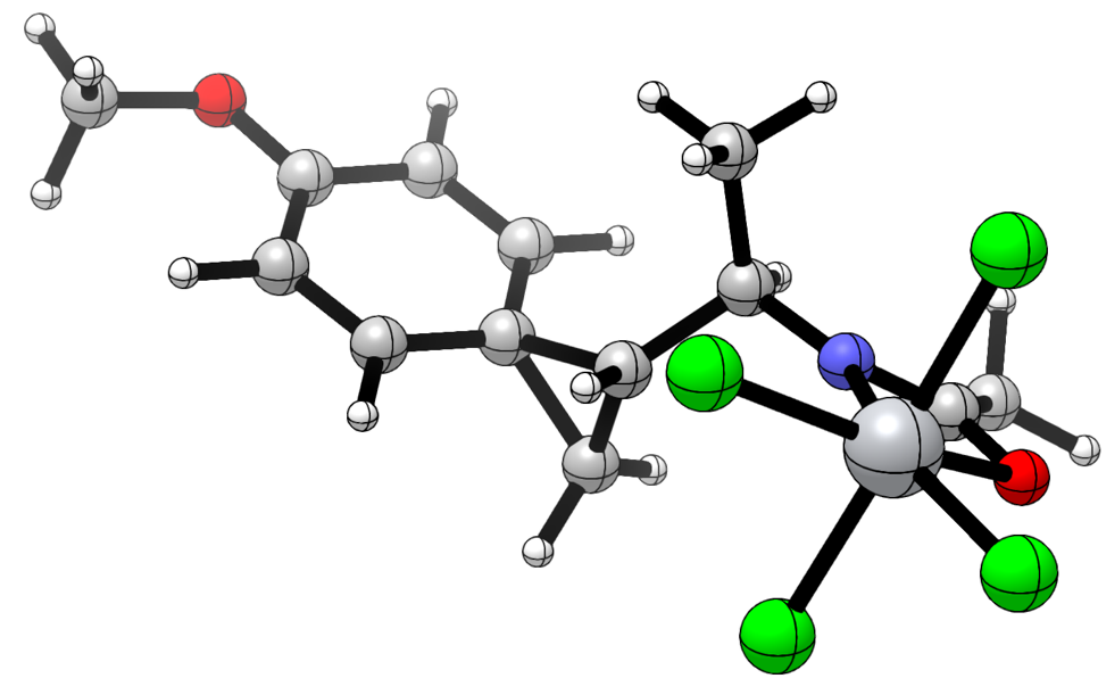

Sum of electronic and thermal free energies: -2608.218028 a.u.

$E_{S P C}\left[\left(I E F-P C M: \mathrm{CH}_{2} \mathrm{Cl}_{2}, \mathrm{SMD}\right) / \mathrm{MN15} /\right.$ def2-QZVPP] $=-3401.233840$ a.u.

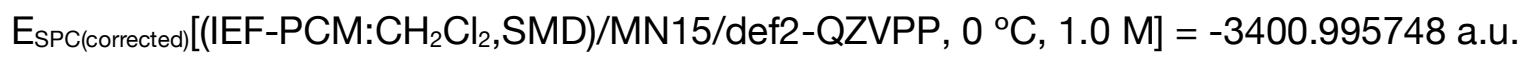

Number of imaginary frequencies: 0

\begin{tabular}{|c|c|c|c|c|c|}
\hline \multirow{2}{*}{$\begin{array}{l}\text { Center } \\
\text { Number }\end{array}$} & \multirow{2}{*}{$\begin{array}{l}\text { Atomic } \\
\text { Number }\end{array}$} & \multirow{2}{*}{$\begin{array}{l}\text { Atomic } \\
\text { Type }\end{array}$} & \multicolumn{3}{|c|}{ Coordinates (Ångstroms) } \\
\hline & & & $\mathrm{X}$ & $\mathrm{Y}$ & $\mathrm{Z}$ \\
\hline 1 & 6 & 0 & -4.60530 & -1.14410 & -0.43730 \\
\hline 2 & 6 & 0 & -3.31660 & -0.97080 & -0.87300 \\
\hline 3 & 6 & 0 & -2.63430 & 0.28560 & -0.73780 \\
\hline 4 & 6 & 0 & -3.35190 & 1.38350 & -0.14150 \\
\hline 5 & 6 & 0 & -4.63840 & 1.22460 & 0.28850 \\
\hline 6 & 6 & 0 & -5.27750 & -0.04080 & 0.15210 \\
\hline 7 & 8 & 0 & -6.51480 & -0.10230 & 0.60170 \\
\hline 8 & 6 & 0 & -7.26100 & -1.32570 & 0.51700 \\
\hline 9 & 6 & 0 & -1.51470 & 0.62270 & -1.80310 \\
\hline 10 & 6 & 0 & -1.05760 & 0.15310 & -0.51700 \\
\hline 11 & 6 & 0 & -0.37020 & 1.05740 & 0.49500 \\
\hline 12 & 6 & 0 & -0.64810 & 0.64070 & 1.93550 \\
\hline 13 & 6 & 0 & 1.80050 & 1.95230 & -0.23760 \\
\hline 14 & 6 & 0 & 1.40330 & 3.36650 & -0.49920 \\
\hline 15 & 8 & 0 & 3.01990 & 1.55090 & -0.44860 \\
\hline 16 & 1 & 0 & -5.09740 & -2.10500 & -0.54180 \\
\hline 17 & 1 & 0 & -2.77720 & -1.79950 & -1.32900 \\
\hline
\end{tabular}




$\begin{array}{rrrrrr}18 & 1 & 0 & -2.86520 & 2.35210 & -0.05160 \\ 19 & 1 & 0 & -5.19990 & 2.04000 & 0.73530 \\ 20 & 1 & 0 & -8.23680 & -1.10390 & 0.94880 \\ 21 & 1 & 0 & -7.37360 & -1.62880 & -0.52870 \\ 22 & 1 & 0 & -6.76530 & -2.11160 & 1.09550 \\ 23 & 1 & 0 & -1.50620 & -0.04520 & -2.66030 \\ 24 & 1 & 0 & -1.45850 & 1.68820 & -2.02120 \\ 25 & 1 & 0 & -0.76350 & -0.89380 & -0.44310 \\ 26 & 1 & 0 & -0.69660 & 2.09490 & 0.33640 \\ 27 & 1 & 0 & -1.72090 & 0.71410 & 2.15530 \\ 28 & 1 & 0 & -0.32190 & -0.39250 & 2.10430 \\ 29 & 1 & 0 & -0.10210 & 1.29370 & 2.62590 \\ 30 & 1 & 0 & 0.97560 & 3.81880 & 0.40330 \\ 31 & 1 & 0 & 2.28120 & 3.93610 & -0.81320 \\ 32 & 1 & 0 & 0.64410 & 3.40310 & -1.29090 \\ 33 & 22 & 0 & 2.63620 & -0.36670 & 0.05270 \\ 34 & 17 & 0 & 3.15000 & 0.15210 & 2.25760 \\ 35 & 17 & 0 & 1.44060 & -2.20680 & 0.70730 \\ 36 & 17 & 0 & 1.96750 & -0.71210 & -2.17710 \\ 37 & 17 & 0 & 4.76200 & -1.04700 & -0.39710 \\ 38 & 7 & 0 & 1.04800 & 0.97460 & 0.19980 \\ ----------------------------------------------------------------------------------------------\end{array}$


Free Energy and Geometry for TiE -TS2-major

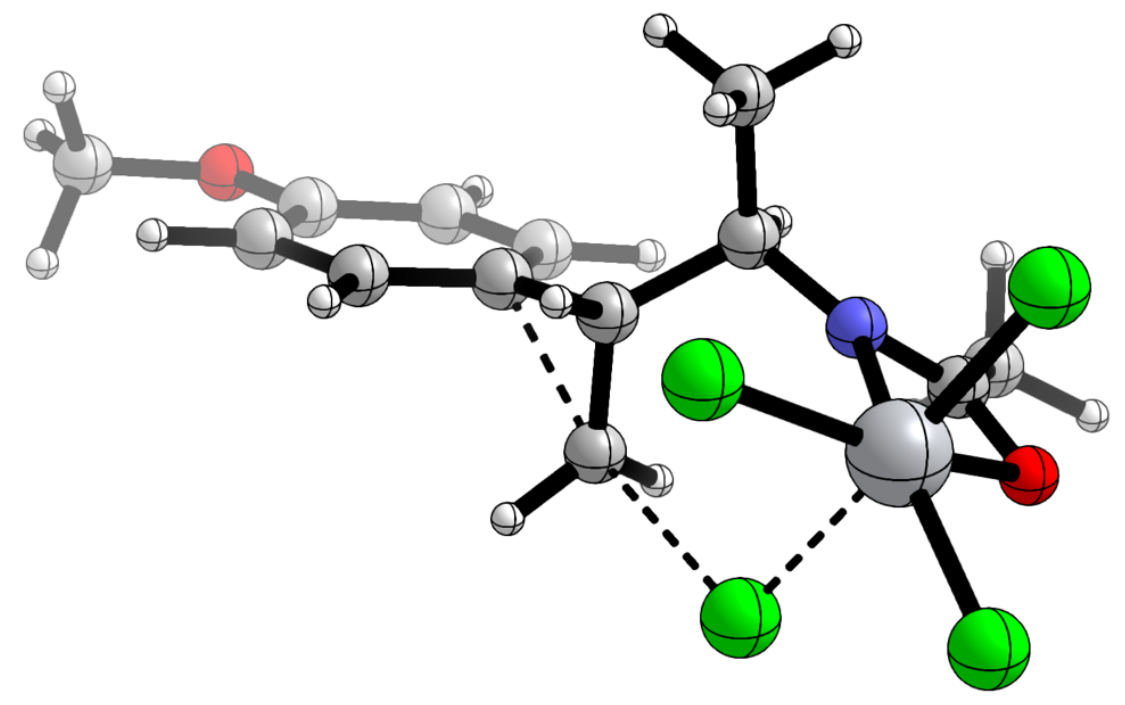

Sum of electronic and thermal free energies: -2608.202306 a.u.

$E_{S P C}\left[\left(I E F-P C M: \mathrm{CH}_{2} \mathrm{Cl}_{2}, \mathrm{SMD}\right) / M N 15 /\right.$ def2-QZVPP] $=-3401.219176$ a.u.

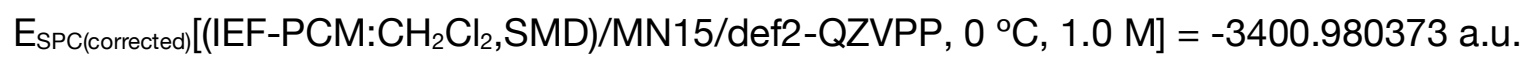

Number of imaginary frequencies: 1

\begin{tabular}{|c|c|c|c|c|c|}
\hline \multirow{2}{*}{$\begin{array}{l}\text { Center } \\
\text { Number }\end{array}$} & \multirow{2}{*}{$\begin{array}{l}\text { Atomic } \\
\text { Number }\end{array}$} & \multirow{2}{*}{$\begin{array}{c}\text { Atomic } \\
\text { Type }\end{array}$} & \multicolumn{3}{|c|}{ Coordinates (Ångstroms) } \\
\hline & & & $\mathrm{X}$ & $\mathrm{Y}$ & $\mathrm{Z}$ \\
\hline 1 & 6 & 0 & -4.73800 & -1.14710 & 0.26500 \\
\hline 2 & 6 & 0 & -3.35580 & -1.22390 & 0.33510 \\
\hline 3 & 6 & 0 & -2.54320 & -0.09240 & 0.09930 \\
\hline 4 & 6 & 0 & -3.16810 & 1.12360 & -0.27190 \\
\hline 5 & 6 & 0 & -4.54540 & 1.20970 & -0.34940 \\
\hline 6 & 6 & 0 & -5.34200 & 0.07970 & -0.07600 \\
\hline 7 & 8 & 0 & -6.66760 & 0.25570 & -0.17240 \\
\hline 8 & 6 & 0 & -7.53500 & -0.84670 & 0.08390 \\
\hline 9 & 6 & 0 & -1.05940 & -0.44370 & -1.15760 \\
\hline 10 & 6 & 0 & -1.01970 & -0.22980 & 0.27580 \\
\hline 11 & 6 & 0 & -0.27340 & 0.99250 & 0.82520 \\
\hline 12 & 6 & 0 & -0.26350 & 0.97710 & 2.34930 \\
\hline 13 & 6 & 0 & 1.56660 & 1.86880 & -0.54540 \\
\hline 14 & 6 & 0 & 0.91530 & 3.10770 & -1.05600 \\
\hline 15 & 8 & 0 & 2.74720 & 1.51300 & -0.95850 \\
\hline 16 & 1 & 0 & -5.33720 & -2.02830 & 0.47110 \\
\hline
\end{tabular}




$\begin{array}{rrrrrr}17 & 1 & 0 & -2.88080 & -2.16910 & 0.59340 \\ 18 & 1 & 0 & -2.57000 & 2.00230 & -0.50410 \\ 19 & 1 & 0 & -5.03560 & 2.13930 & -0.62640 \\ 20 & 1 & 0 & -8.54950 & -0.46890 & -0.05170 \\ 21 & 1 & 0 & -7.34750 & -1.66170 & -0.62520 \\ 22 & 1 & 0 & -7.41060 & -1.20770 & 1.11180 \\ 23 & 1 & 0 & -1.32460 & -1.41630 & -1.55780 \\ 24 & 1 & 0 & -1.07760 & 0.40990 & -1.83330 \\ 25 & 1 & 0 & -0.79880 & -1.13830 & 0.84120 \\ 26 & 1 & -0.77010 & 1.90420 & 0.47090 \\ 27 & 1 & 0 & -1.29010 & 0.95750 & 2.73590 \\ 28 & 1 & 0 & 0.26830 & 0.09010 & 2.71670 \\ 29 & 1 & 0 & 0.24030 & 1.87130 & 2.73340 \\ 30 & 1 & 0.48720 & 3.69410 & -0.23590 \\ 31 & 1 & 0 & 1.65120 & 3.70610 & -1.59850 \\ 32 & 1 & 0 & 0.10130 & 2.83330 & -1.74160 \\ 33 & 0 & 2.69780 & -0.24590 & 0.01170 \\ 34 & 22 & 0 & 3.82600 & 0.64580 & 1.74790 \\ 35 & 17 & 0 & 1.84250 & -1.96340 & 1.21890 \\ 36 & 17 & 0 & 1.27510 & -0.98620 & -1.92370 \\ 37 & 17 & 0 & 4.48470 & -1.07540 & -1.09410 \\ 38 & 17 & 0 & 1.07770 & 0.99070 & 0.29900 \\ --------------------------------------------------------------------\end{array}$


Free Energy and Geometry for TiE -TS2-minor

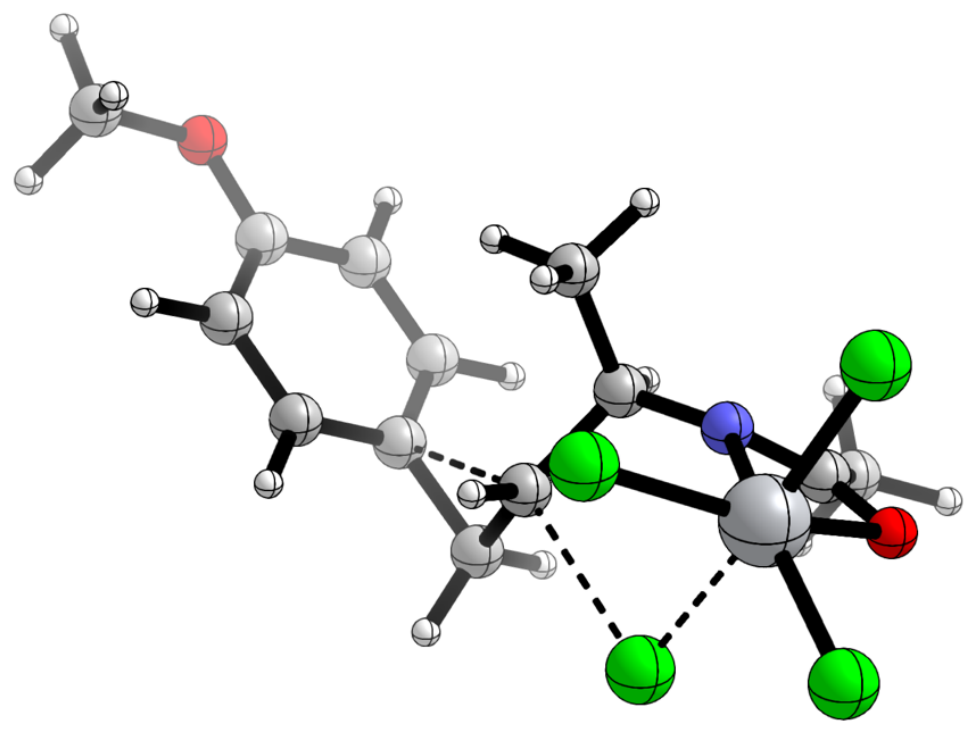

Sum of electronic and thermal free energies: -2608.197819 a.u.

$E_{\text {sPC }}\left[\left(I E F-P C M: \mathrm{CH}_{2} \mathrm{Cl}_{2}, \mathrm{SMD}\right) / \mathrm{MN15} /\right.$ def2-QZVPP] $=-3401.215678$ a.u.

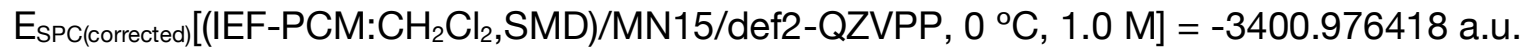

Number of imaginary frequencies: 1

\begin{tabular}{cccccc}
$\begin{array}{c}\text { Center } \\
\text { Number }\end{array}$ & $\begin{array}{c}\text { Atomic } \\
\text { Number }\end{array}$ & $\begin{array}{c}\text { Atomic } \\
\text { Type }\end{array}$ & \multicolumn{3}{c}{ Coordinates (Angstroms) } \\
& & & $\mathrm{X}$ & $\mathrm{Y}$ & $\mathrm{Z}$ \\
\hdashline 1 & 6 & 0 & -4.43860 & -0.81930 & -0.84400 \\
- & 6 & 0 & -3.28810 & -0.28160 & -1.41640 \\
3 & 6 & 0 & -2.74930 & 0.93710 & -0.96370 \\
4 & 6 & 0 & -3.38570 & 1.60970 & 0.10610 \\
5 & 6 & 0 & -4.53420 & 1.09330 & 0.67180 \\
6 & 6 & 0 & -5.06420 & -0.13230 & 0.20810 \\
7 & 8 & 0 & -6.17390 & -0.56250 & 0.83110 \\
8 & 6 & 0 & -6.76420 & -1.79170 & 0.41800 \\
9 & 6 & 0 & -1.45910 & 1.48490 & -1.57070 \\
10 & 6 & 0 & -0.72320 & 0.48240 & -0.80860 \\
11 & 6 & 0 & -0.41700 & 0.61420 & 0.68240 \\
12 & 6 & 0 & -1.05780 & -0.52680 & 1.47640 \\
13 & 6 & 0 & 1.75020 & 1.68460 & 1.02970 \\
14 & 6 & 0 & 1.27930 & 3.05780 & 1.35530 \\
15 & 8 & 0 & 3.00120 & 1.44350 & 0.79760
\end{tabular}




\begin{tabular}{rrrrrr}
16 & 1 & 0 & -4.83550 & -1.75860 & -1.21580 \\
17 & 1 & 0 & -2.80150 & -0.80970 & -2.23510 \\
18 & 1 & 0 & -2.97480 & 2.55020 & 0.46930 \\
19 & 1 & 0 & -5.04770 & 1.60940 & 1.47920 \\
20 & 1 & 0 & -7.63800 & -1.93630 & 1.05530 \\
21 & 1 & 0 & -7.07870 & -1.73890 & -0.63130 \\
22 & 1 & 0 & -6.06510 & -2.62520 & 0.55710 \\
23 & 1 & 0 & -1.39930 & 1.35780 & -2.65250 \\
24 & 1 & 0 & -1.27330 & 2.51460 & -1.25450 \\
25 & 1 & 0 & -0.64620 & -0.52090 & -1.22930 \\
26 & 1 & 0 & -0.80840 & 1.58140 & 1.02510 \\
27 & 1 & 0 & -2.15000 & -0.51280 & 1.38480 \\
28 & 1 & 0 & -0.68380 & -1.49430 & 1.12240 \\
29 & 1 & 0 & -0.78910 & -0.41160 & 2.53270 \\
30 & 1 & 0 & 0.51250 & 3.04250 & 2.13650 \\
31 & 1 & 0 & 2.13040 & 3.66550 & 1.67220 \\
32 & 1 & 0 & 0.84250 & 3.50360 & 0.45000 \\
33 & 22 & 0 & 2.63650 & -0.39830 & 0.05110 \\
34 & 17 & 0 & 3.33720 & -1.29220 & 1.98290 \\
35 & 17 & 0 & 1.46180 & -2.24480 & -0.55490 \\
36 & 17 & 0 & 1.58220 & 0.77070 & -1.91340 \\
37 & 17 & 0 & 4.56230 & -0.46590 & -1.10120 \\
38 & 7 & 0 & 1.02250 & 0.59620 & 0.86940 \\
\hline & 17 & & & &
\end{tabular}


Free Energy and Geometry for TiE -Int3-major

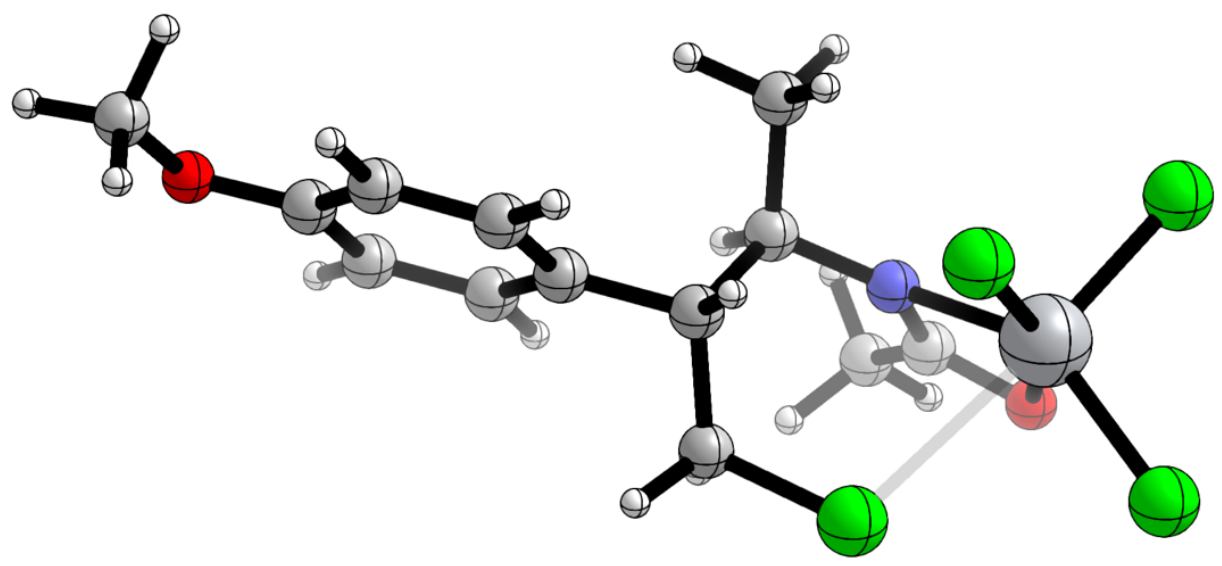

Sum of electronic and thermal free energies: -2608.229631 a.u.

$E_{S P C}\left[\left(I E F-P C M: \mathrm{CH}_{2} \mathrm{Cl}_{2}, \mathrm{SMD}\right) / \mathrm{MN15} /\right.$ def2-QZVPP] $=-3401.249916$ a.u.

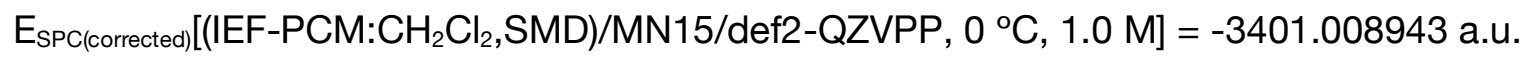

Number of imaginary frequencies: 0

\begin{tabular}{|c|c|c|c|c|c|}
\hline \multirow{2}{*}{$\begin{array}{l}\text { Center } \\
\text { Number }\end{array}$} & \multirow{2}{*}{$\begin{array}{l}\text { Atomic } \\
\text { Number }\end{array}$} & \multirow{2}{*}{$\begin{array}{l}\text { Atomic } \\
\text { Type }\end{array}$} & \multicolumn{3}{|c|}{ Coordinates (Ångstroms) } \\
\hline & & & $\mathrm{X}$ & Y & Z \\
\hline 1 & 6 & 0 & 4.66390 & -1.16770 & -0.01630 \\
\hline 2 & 6 & 0 & 3.26820 & -1.20900 & 0.09020 \\
\hline 3 & 6 & 0 & 2.50760 & -0.04320 & 0.20450 \\
\hline 4 & 6 & 0 & 3.18240 & 1.19100 & 0.21330 \\
\hline 5 & 6 & 0 & 4.56800 & 1.25110 & 0.11460 \\
\hline 6 & 6 & 0 & 5.31860 & 0.06980 & -0.00280 \\
\hline 7 & 8 & 0 & 6.66540 & 0.22070 & -0.09800 \\
\hline 8 & 6 & 0 & 7.46280 & -0.94570 & -0.22830 \\
\hline 9 & 6 & 0 & 0.57860 & 0.55150 & 1.63370 \\
\hline 10 & 6 & 0 & 0.99520 & -0.10920 & 0.31650 \\
\hline 11 & 6 & 0 & 0.33940 & 0.55870 & -0.91700 \\
\hline 12 & 6 & 0 & 0.56060 & -0.23560 & -2.19790 \\
\hline 13 & 6 & 0 & -1.66200 & 1.90290 & -0.40590 \\
\hline 14 & 6 & 0 & -1.08940 & 3.26970 & -0.52740 \\
\hline 15 & 8 & 0 & -2.87600 & 1.71720 & 0.03420 \\
\hline 16 & 1 & 0 & 5.22000 & -2.09630 & -0.10730 \\
\hline 17 & 1 & 0 & 2.76490 & -2.17530 & 0.07950 \\
\hline
\end{tabular}




$\begin{array}{rrrrrr}18 & 1 & 0 & 2.61760 & 2.11990 & 0.30010 \\ 19 & 1 & 0 & 5.09090 & 2.20500 & 0.12380 \\ 20 & 1 & 0 & 8.49810 & -0.60440 & -0.29200 \\ 21 & 1 & 0 & 7.34890 & -1.60230 & 0.64440 \\ 22 & 1 & 0 & 7.20580 & -1.50040 & -1.14040 \\ 23 & 1 & 0 & 1.23320 & 0.23980 & 2.45000 \\ 24 & 1 & 0 & 0.57810 & 1.64450 & 1.56720 \\ 25 & 1 & 0 & 0.70020 & -1.16660 & 0.34500 \\ 26 & 1 & 0 & 0.78720 & 1.55870 & -1.02570 \\ 27 & 1 & 0 & 1.63180 & -0.31240 & -2.41810 \\ 28 & 1 & 0 & 0.15200 & -1.24920 & -2.10120 \\ 29 & 1 & 0 & -0.54960 & 3.38260 & -1.47320 \\ 30 & 1 & 0 & -1.89500 & 4.00470 & -0.45970 \\ 31 & 1 & 0 & -0.37890 & 3.43960 & 0.29310 \\ 32 & 1 & 0 & -2.75440 & -0.26280 & -0.12470 \\ 33 & 22 & 0 & -3.87530 & -0.37650 & -2.00140 \\ 34 & 17 & 0 & -1.75700 & -2.27110 & -0.21340 \\ 35 & 17 & 0 & -1.09050 & 0.07930 & 2.19740 \\ 36 & 17 & 0 & -4.29890 & -0.58690 & 1.46140 \\ 37 & 17 & 0 & -1.08360 & 0.75150 & -0.67220 \\ 38 & 7 & 0 .-10 & \\ -----------------------------------------------------------------------------------------------\end{array}$


Free Energy and Geometry for TiE -Int3-minor

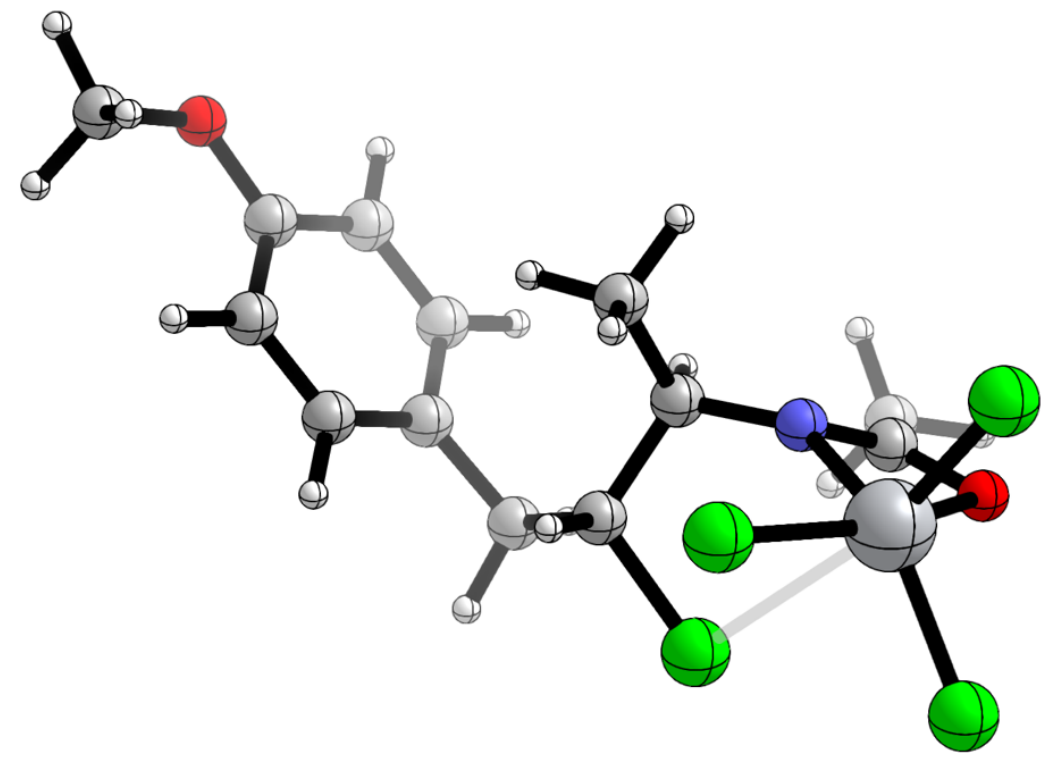

Sum of electronic and thermal free energies: -2608.227076 a.u.

$E_{s P C}\left[\left(I E F-P C M: \mathrm{CH}_{2} \mathrm{Cl}_{2}, \mathrm{SMD}\right) / \mathrm{MN15} /\right.$ def2-QZVPP] $=-3401.245905$ a.u.

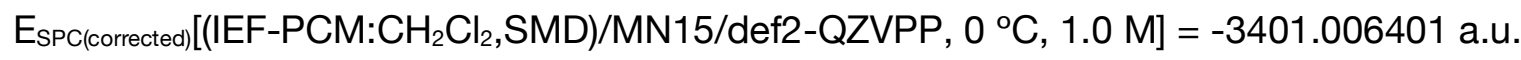

Number of imaginary frequencies: 0

\begin{tabular}{|c|c|c|c|c|c|}
\hline \multirow{2}{*}{$\begin{array}{l}\text { Center } \\
\text { Number }\end{array}$} & \multirow{2}{*}{$\begin{array}{l}\text { Atomic } \\
\text { Number }\end{array}$} & \multirow{2}{*}{$\begin{array}{c}\text { Atomic } \\
\text { Type }\end{array}$} & \multicolumn{3}{|c|}{ Coordinates (Ångstroms) } \\
\hline & & & $\mathrm{X}$ & Y & $\mathrm{Z}$ \\
\hline 1 & 6 & 0 & 4.70040 & 0.60330 & -0.93350 \\
\hline 2 & 6 & 0 & 3.54760 & -0.04690 & -1.38620 \\
\hline 3 & 6 & 0 & 2.93530 & -1.05850 & -0.63960 \\
\hline 4 & 6 & 0 & 3.51980 & -1.42620 & 0.58230 \\
\hline 5 & 6 & 0 & 4.66850 & -0.79350 & 1.04890 \\
\hline 6 & 6 & 0 & 5.26190 & 0.23180 & 0.29630 \\
\hline 7 & 8 & 0 & 6.37680 & 0.80020 & 0.82630 \\
\hline 8 & 6 & 0 & 7.01000 & 1.84150 & 0.09980 \\
\hline 9 & 6 & 0 & 1.63400 & -1.67090 & -1.10040 \\
\hline 10 & 6 & 0 & 0.45420 & -0.71390 & -0.89290 \\
\hline 11 & 6 & 0 & 0.23930 & -0.32140 & 0.59920 \\
\hline 12 & 6 & 0 & 0.92630 & 0.99670 & 0.95310 \\
\hline 13 & 6 & 0 & -1.85000 & -1.24040 & 1.46570 \\
\hline
\end{tabular}




\begin{tabular}{|c|c|c|c|c|c|}
\hline 14 & 6 & 0 & -1.30370 & -2.46680 & 2.10070 \\
\hline 15 & 8 & 0 & -3.13000 & -1.06390 & 1.33070 \\
\hline 16 & 1 & 0 & 5.14670 & 1.38620 & -1.54020 \\
\hline 17 & 1 & 0 & 3.11180 & 0.25270 & -2.33970 \\
\hline 18 & 1 & 0 & 3.06170 & -2.21510 & 1.17880 \\
\hline 19 & 1 & 0 & 5.12230 & -1.07800 & 1.99600 \\
\hline 20 & 1 & 0 & 7.86800 & 2.15540 & 0.69780 \\
\hline 21 & 1 & 0 & 7.35920 & 1.48600 & -0.87860 \\
\hline 22 & 1 & 0 & 6.33270 & 2.69440 & -0.04070 \\
\hline 23 & 1 & 0 & 1.68560 & -1.92310 & -2.16690 \\
\hline 24 & 1 & 0 & 1.43220 & -2.59610 & -0.54270 \\
\hline 25 & 1 & 0 & 0.57140 & 0.18760 & -1.50370 \\
\hline 26 & 1 & 0 & 0.66940 & -1.12740 & 1.21320 \\
\hline 27 & 1 & 0 & 2.00740 & 0.93910 & 0.78910 \\
\hline 28 & 1 & 0 & 0.52500 & 1.81620 & 0.34650 \\
\hline 29 & 1 & 0 & 0.74690 & 1.22290 & 2.01070 \\
\hline 30 & 1 & 0 & -0.52350 & -2.21160 & 2.82590 \\
\hline 31 & 1 & 0 & -2.11410 & -3.01250 & 2.58890 \\
\hline 32 & 1 & 0 & -0.85270 & -3.10290 & 1.32600 \\
\hline 33 & 22 & 0 & -2.88270 & 0.48370 & 0.09900 \\
\hline 34 & 17 & 0 & -3.75900 & 1.94520 & 1.45110 \\
\hline 35 & 17 & 0 & -1.74280 & 1.85650 & -1.25440 \\
\hline 36 & 17 & 0 & -1.02590 & -1.53580 & -1.57040 \\
\hline 37 & 17 & 0 & -4.46750 & -0.22720 & -1.27410 \\
\hline 38 & 7 & 0 & -1.17830 & -0.23860 & 0.92290 \\
\hline
\end{tabular}


Free Energy and Geometry for TiZ -Int1

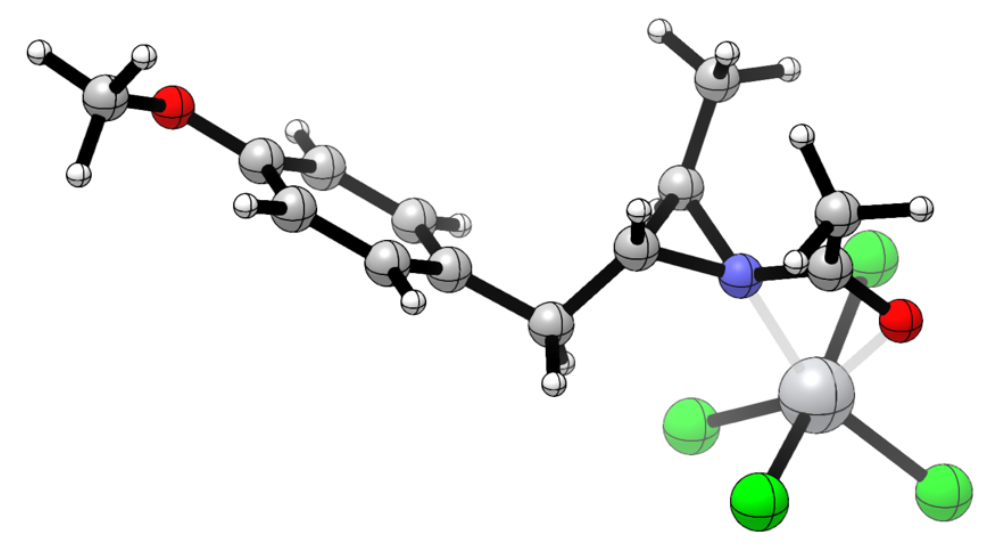

Sum of electronic and thermal free energies: -2608.220910 a.u.

$E_{\text {SPC }}\left[\left(I E F-P C M: \mathrm{CH}_{2} \mathrm{Cl}_{2}, \mathrm{SMD}\right) / \mathrm{MN15} /\right.$ def2-QZVPP] $=-3401.236339$ a.u.

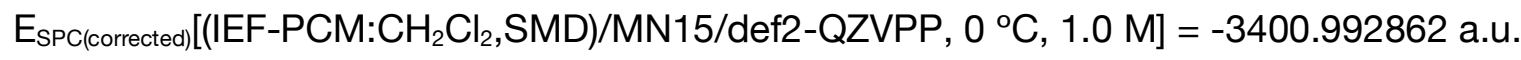

Number of imaginary frequencies: 0

\begin{tabular}{|c|c|c|c|c|c|}
\hline \multirow{2}{*}{$\begin{array}{l}\text { Center } \\
\text { Number }\end{array}$} & \multirow{2}{*}{$\begin{array}{l}\text { Atomic } \\
\text { Number }\end{array}$} & \multirow{2}{*}{$\begin{array}{c}\text { Atomic } \\
\text { Type }\end{array}$} & \multicolumn{3}{|c|}{ Coordinates (Ångstroms) } \\
\hline & & & $\mathrm{X}$ & $\mathrm{Y}$ & $\mathrm{Z}$ \\
\hline 1 & 6 & 0 & 4.43840 & -0.81690 & 1.17340 \\
\hline 2 & 6 & 0 & 3.09290 & -0.82080 & 0.82230 \\
\hline 3 & 6 & 0 & 2.67610 & -0.41880 & -0.45870 \\
\hline 4 & 6 & 0 & 3.65130 & -0.01180 & -1.37180 \\
\hline 5 & 6 & 0 & 5.01150 & -0.00160 & -1.03700 \\
\hline 6 & 6 & 0 & 5.40730 & -0.40430 & 0.24390 \\
\hline 7 & 8 & 0 & 6.69860 & -0.43270 & 0.66870 \\
\hline 8 & 6 & 0 & 7.71120 & -0.02760 & -0.23820 \\
\hline 9 & 6 & 0 & 1.20500 & -0.38890 & -0.81210 \\
\hline 10 & 6 & 0 & 0.59260 & 0.89980 & -0.31100 \\
\hline 11 & 6 & 0 & 0.10580 & 1.07930 & 1.06800 \\
\hline 12 & 6 & 0 & 0.20960 & 2.35960 & 1.84730 \\
\hline 13 & 6 & 0 & -1.70460 & 1.93770 & -0.60070 \\
\hline 14 & 6 & 0 & -1.23850 & 3.20230 & -1.21470 \\
\hline 15 & 8 & 0 & -2.89620 & 1.58630 & -0.61760 \\
\hline 16 & 1 & 0 & 4.76300 & -1.13510 & 2.16190 \\
\hline
\end{tabular}




$\begin{array}{rrrrrr}17 & 1 & 0 & 2.34920 & -1.15080 & 1.54820 \\ 18 & 1 & 0 & 3.35090 & 0.29980 & -2.37200 \\ 19 & 1 & 0 & 5.74170 & 0.31520 & -1.77630 \\ 20 & 1 & 0 & 7.57360 & 1.01810 & -0.54430 \\ 21 & 1 & 0 & 8.65890 & -0.12640 & 0.29530 \\ 22 & 1 & 0 & 7.72620 & -0.67080 & -1.12810 \\ 23 & 1 & 0 & 1.06690 & -0.45430 & -1.89770 \\ 24 & 1 & 0 & 0.68210 & -1.23600 & -0.35230 \\ 25 & 1 & 0 & 0.95100 & 1.80760 & -0.80110 \\ 26 & 1 & 0 & 0.05330 & 0.17300 & 1.67450 \\ 27 & 1 & 0 & 1.02350 & 2.24320 & 2.57240 \\ 28 & 1 & 0 & 0.44330 & 3.22100 & 1.21540 \\ 29 & 1 & 0 & -0.71490 & 2.55290 & 2.40380 \\ 30 & 1 & 0 & -0.79650 & 2.94620 & -2.18950 \\ 31 & 1 & 0 & -2.09480 & 3.86080 & -1.37380 \\ 32 & 1 & 0 & -0.46790 & 3.70050 & -0.62140 \\ 33 & 7 & 0 & -0.88260 & 0.92440 & -0.07090 \\ 34 & 22 & 0 & -2.64130 & -0.47540 & -0.00020 \\ 35 & 17 & 0 & -1.61740 & -2.28060 & 0.77890 \\ 36 & 17 & 0 & -2.93590 & 0.42870 & 2.08140 \\ 37 & 17 & 0 & -4.77080 & -0.98500 & -0.29360 \\ 38 & 17 & 0 & -2.05210 & -0.81810 & -2.17770 \\ --------------------------------------------------------------------------------\end{array}$




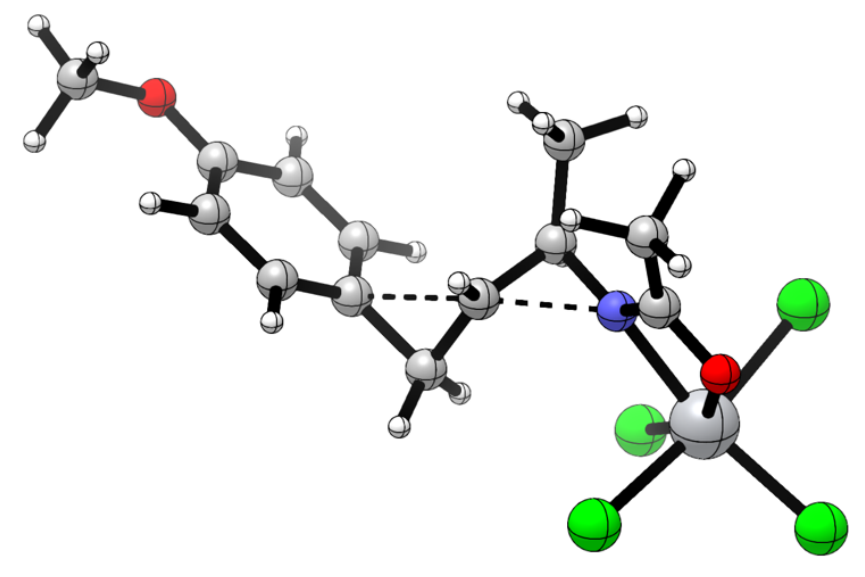

Sum of electronic and thermal free energies: -2608.198800 a.u.

$E_{\text {sPC }}\left[\left(I E F-P C M: \mathrm{CH}_{2} \mathrm{Cl}_{2}, \mathrm{SMD}\right) / \mathrm{MN15} /\right.$ def2-QZVPP] $=-3401.215602$ a.u.

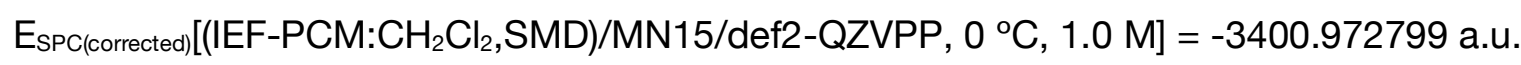

Number of imaginary frequencies: 1

\begin{tabular}{|c|c|c|c|c|c|}
\hline \multirow{2}{*}{$\begin{array}{l}\text { Center } \\
\text { Number }\end{array}$} & \multirow{2}{*}{$\begin{array}{l}\text { Atomic } \\
\text { Number }\end{array}$} & \multirow{2}{*}{$\begin{array}{l}\text { Atomic } \\
\text { Type }\end{array}$} & \multicolumn{3}{|c|}{ Coordinates (Ångstroms) } \\
\hline & & & $\mathrm{X}$ & Y & Z \\
\hline 1 & 6 & 0 & 4.38890 & -0.59270 & 1.33670 \\
\hline 2 & 6 & 0 & 3.07080 & -0.85260 & 1.00460 \\
\hline 3 & 6 & 0 & 2.65680 & -0.83830 & -0.34520 \\
\hline 4 & 6 & 0 & 3.59040 & -0.50250 & -1.33940 \\
\hline 5 & 6 & 0 & 4.91980 & -0.23790 & -1.01720 \\
\hline 6 & 6 & 0 & 5.32320 & -0.28420 & 0.32760 \\
\hline 7 & 8 & 0 & 6.58270 & -0.04510 & 0.74220 \\
\hline 8 & 6 & 0 & 7.57650 & 0.26670 & -0.22760 \\
\hline 9 & 6 & 0 & 1.19980 & -1.13830 & -0.71250 \\
\hline 10 & 6 & 0 & 0.91040 & 0.28770 & -0.59130 \\
\hline 11 & 6 & 0 & 0.35290 & 0.93340 & 0.61420 \\
\hline 12 & 6 & 0 & 0.88000 & 2.28590 & 1.04690 \\
\hline 13 & 6 & 0 & -1.53160 & 1.75660 & -0.88480 \\
\hline 14 & 6 & 0 & -1.02170 & 3.05300 & -1.40920 \\
\hline 15 & 8 & 0 & -2.72710 & 1.38630 & -1.13980 \\
\hline 16 & 1 & 0 & 4.72620 & -0.62140 & 2.36990 \\
\hline 17 & 1 & 0 & 2.34910 & -1.09260 & 1.78450 \\
\hline 18 & 1 & 0 & 3.27230 & -0.46710 & -2.38040 \\
\hline 19 & 1 & 0 & 5.62560 & 0.00220 & -1.80620 \\
\hline 20 & 1 & 0 & 7.32300 & 1.18890 & -0.76490 \\
\hline & & S-130 & & & \\
\hline
\end{tabular}




$\begin{array}{rrrrrr}21 & 1 & 0 & 8.50570 & 0.41110 & 0.32620 \\ 22 & 1 & 0 & 7.69880 & -0.55780 & -0.94090 \\ 23 & 1 & 0 & 1.10030 & -1.53760 & -1.72260 \\ 24 & 1 & 0 & 0.70620 & -1.76150 & 0.03620 \\ 25 & 1 & 0 & 1.15070 & 0.94060 & -1.43240 \\ 26 & 1 & 0 & 0.28880 & 0.24070 & 1.46010 \\ 27 & 1 & 0 & 1.78220 & 2.12490 & 1.64890 \\ 28 & 1 & 0 & 1.14510 & 2.92390 & 0.19880 \\ 29 & 1 & 0 & 0.13500 & 2.79650 & 1.66850 \\ 30 & 1 & 0 & 0.00490 & 2.96860 & -1.77800 \\ 31 & 1 & 0 & -1.68380 & 3.39060 & -2.21040 \\ 32 & 1 & 0 & -1.03790 & 3.79360 & -0.59840 \\ 33 & 7 & 0 & -0.92290 & 0.85090 & -0.10110 \\ 34 & 22 & 0 & -2.71300 & -0.30270 & 0.05660 \\ 35 & 17 & 0 & -1.78840 & -1.84180 & 1.42450 \\ 36 & 17 & 0 & -3.15540 & 1.13110 & 1.83300 \\ 37 & 17 & 0 & -4.88310 & -0.79040 & -0.23960 \\ 38 & 17 & 0 & -2.03710 & -1.41610 & -1.84330 \\ -----0.040\end{array}$




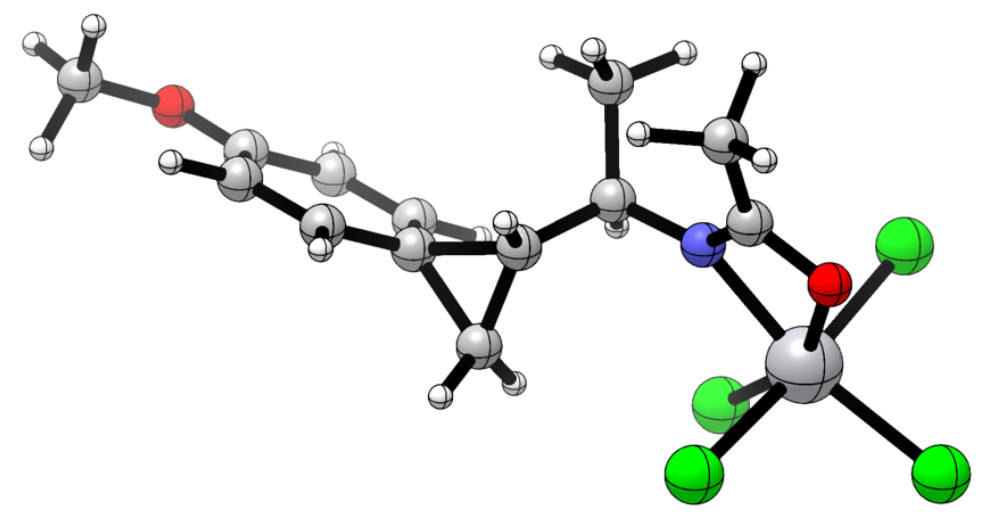

Sum of electronic and thermal free energies: -2608.214828 a.u.

$\mathrm{E}_{\mathrm{SPC}}\left[\left(\mathrm{IEF}-\mathrm{PCM}: \mathrm{CH}_{2} \mathrm{Cl}_{2}, \mathrm{SMD}\right) / \mathrm{MN} 15 /\right.$ def2-QZVPP] $=-3401.230882$ a.u.

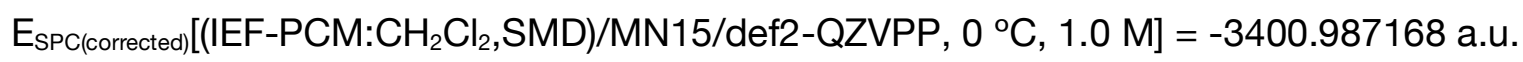

Number of imaginary frequencies: 0

\begin{tabular}{|c|c|c|c|c|c|}
\hline \multirow{2}{*}{$\begin{array}{l}\text { Center } \\
\text { Number }\end{array}$} & \multirow{2}{*}{$\begin{array}{l}\text { Atomic } \\
\text { Number }\end{array}$} & \multirow{2}{*}{$\begin{array}{c}\text { Atomic } \\
\text { Type }\end{array}$} & \multicolumn{3}{|c|}{ Coordinates (Ångstroms) } \\
\hline & & & $X$ & $\mathrm{Y}$ & $\mathrm{Z}$ \\
\hline 1 & 6 & 0 & -4.26940 & -0.87330 & -1.18110 \\
\hline 2 & 6 & 0 & -2.96100 & -0.83940 & -0.78950 \\
\hline 3 & 6 & 0 & -2.59170 & -0.30670 & 0.49680 \\
\hline 4 & 6 & 0 & -3.63940 & 0.17250 & 1.35220 \\
\hline 5 & 6 & 0 & -4.95580 & 0.14790 & 0.96770 \\
\hline 6 & 6 & 0 & -5.28020 & -0.37230 & -0.31300 \\
\hline 7 & 8 & 0 & -6.51160 & -0.43370 & -0.78030 \\
\hline 8 & 6 & 0 & -7.61430 & 0.03800 & 0.00810 \\
\hline 9 & 6 & 0 & -1.29840 & -0.89680 & 1.19940 \\
\hline 10 & 6 & 0 & -1.19030 & 0.44380 & 0.67580 \\
\hline 11 & 6 & 0 & -0.37180 & 0.76770 & -0.56550 \\
\hline 12 & 6 & 0 & -0.91570 & 1.96740 & -1.33830 \\
\hline 13 & 6 & 0 & 1.63330 & 1.90610 & 0.43210 \\
\hline 14 & 6 & 0 & 1.12910 & 3.25930 & 0.80400 \\
\hline 15 & 8 & 0 & 2.87730 & 1.59520 & 0.66560 \\
\hline 16 & 1 & 0 & -4.57000 & -1.27830 & -2.14320 \\
\hline 17 & 1 & 0 & -2.18880 & -1.23850 & -1.44390 \\
\hline 18 & 1 & 0 & -3.36420 & 0.56670 & 2.32920 \\
\hline 19 & 1 & 0 & -5.72950 & 0.51920 & 1.63120 \\
\hline 20 & 1 & 0 & -7.49520 & 1.10430 & 0.22600 \\
\hline 21 & 1 & 0 & -8.50180 & -0.12110 & -0.60420 \\
\hline
\end{tabular}




\begin{tabular}{|c|c|c|c|c|c|}
\hline 22 & 1 & 0 & -7.69030 & -0.53910 & 0.93510 \\
\hline 23 & 1 & 0 & -1.41520 & -1.05720 & 2.26760 \\
\hline 24 & 1 & 0 & -0.85110 & -1.71200 & 0.63370 \\
\hline 25 & 1 & 0 & -1.28240 & 1.27090 & 1.38110 \\
\hline 26 & 1 & 0 & -0.37360 & -0.10500 & -1.22810 \\
\hline 27 & 1 & 0 & -1.86440 & 1.69110 & -1.81580 \\
\hline 28 & 1 & 0 & -1.10320 & 2.82830 & -0.68740 \\
\hline 29 & 1 & 0 & -0.20840 & 2.26130 & -2.12310 \\
\hline 30 & 1 & 0 & 0.12020 & 3.20690 & 1.22540 \\
\hline 31 & 1 & 0 & 1.81460 & 3.70540 & 1.52950 \\
\hline 32 & 1 & 0 & 1.09870 & 3.89450 & -0.09070 \\
\hline 33 & 7 & 0 & 1.00970 & 0.90320 & -0.13560 \\
\hline 34 & 22 & 0 & 2.70430 & -0.28120 & -0.03170 \\
\hline 35 & 17 & 0 & 1.61080 & -2.08030 & -0.94160 \\
\hline 36 & 17 & 0 & 3.25470 & 0.51770 & -2.15520 \\
\hline 37 & 17 & 0 & 4.87150 & -0.86100 & 0.39040 \\
\hline 38 & 17 & 0 & 2.03190 & -0.92400 & 2.12110 \\
\hline
\end{tabular}




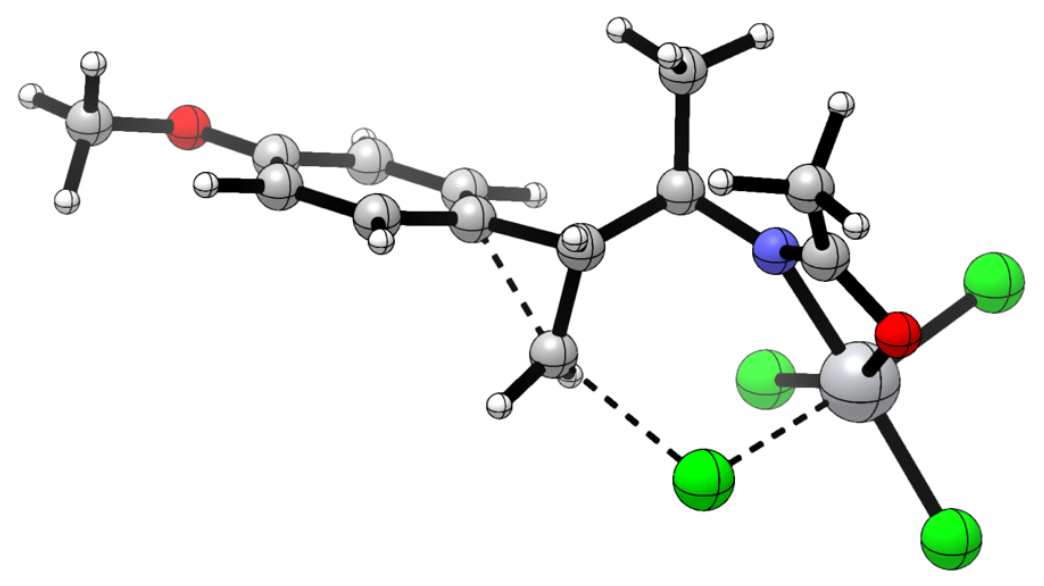

Sum of electronic and thermal free energies: -2608.199576 a.u.

$E_{\text {SPC }}\left[\left(I E F-P C M: \mathrm{CH}_{2} \mathrm{Cl}_{2}, \mathrm{SMD}\right) / \mathrm{MN15} /\right.$ def2-QZVPP] $=-3401.218237$ a.u.

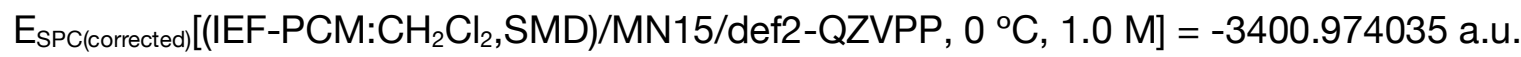

Number of imaginary frequencies: 1

\begin{tabular}{|c|c|c|c|c|c|}
\hline \multirow{2}{*}{$\begin{array}{l}\text { Center } \\
\text { Number }\end{array}$} & \multirow{2}{*}{$\begin{array}{l}\text { Atomic } \\
\text { Number }\end{array}$} & \multirow{2}{*}{$\begin{array}{l}\text { Atomic } \\
\text { Type }\end{array}$} & \multicolumn{3}{|c|}{ Coordinates (Ångstroms) } \\
\hline & & & $\mathrm{X}$ & $\mathrm{Y}$ & $\mathrm{Z}$ \\
\hline 1 & 6 & 0 & 4.20530 & -0.82770 & 1.19490 \\
\hline 2 & 6 & 0 & 2.88560 & -0.50320 & 0.94810 \\
\hline 3 & 6 & 0 & 2.53550 & 0.25260 & -0.19910 \\
\hline 4 & 6 & 0 & 3.55390 & 0.61880 & -1.10540 \\
\hline 5 & 6 & 0 & 4.88180 & 0.29780 & -0.86670 \\
\hline 6 & 6 & 0 & 5.21410 & -0.42760 & 0.29400 \\
\hline 7 & 8 & 0 & 6.46510 & -0.78740 & 0.61460 \\
\hline 8 & 6 & 0 & 7.53660 & -0.42450 & -0.25320 \\
\hline 9 & 6 & 0 & 0.99840 & -0.58330 & -1.10180 \\
\hline 10 & 6 & 0 & 1.09420 & 0.71940 & -0.47080 \\
\hline 11 & 6 & 0 & 0.22460 & 1.02160 & 0.75330 \\
\hline 12 & 6 & 0 & 0.67660 & 2.29510 & 1.46510 \\
\hline 13 & 6 & 0 & -1.83420 & 1.92010 & -0.36490 \\
\hline 14 & 6 & 0 & -1.45250 & 3.30680 & -0.75270 \\
\hline 15 & 8 & 0 & -2.99260 & 1.44020 & -0.72170 \\
\hline 16 & 1 & 0 & 4.48840 & -1.39910 & 2.07510 \\
\hline 17 & 1 & 0 & 2.11760 & -0.83370 & 1.64470 \\
\hline 18 & 1 & 0 & 3.28740 & 1.17640 & -2.00190 \\
\hline 19 & 1 & 0 & 5.64590 & 0.60530 & -1.57360 \\
\hline
\end{tabular}




\begin{tabular}{|c|c|c|c|c|c|}
\hline 20 & 1 & 0 & 7.61070 & 0.66570 & -0.34330 \\
\hline 21 & 1 & 0 & 8.44380 & -0.81710 & 0.20840 \\
\hline 22 & 1 & 0 & 7.40300 & -0.87440 & -1.24420 \\
\hline 23 & 1 & 0 & 1.35290 & -0.72140 & -2.11810 \\
\hline 24 & 1 & 0 & 0.81000 & -1.46910 & -0.49900 \\
\hline 25 & 1 & 0 & 1.09810 & 1.54970 & -1.18400 \\
\hline 26 & 1 & 0 & 0.30000 & 0.18570 & 1.45630 \\
\hline 27 & 1 & 0 & 1.64070 & 2.11100 & 1.95470 \\
\hline 28 & 1 & 0 & 0.80370 & 3.13270 & 0.77030 \\
\hline 29 & 1 & 0 & -0.05130 & 2.57660 & 2.23440 \\
\hline 30 & 1 & 0 & -0.44860 & 3.32850 & -1.19060 \\
\hline 31 & 1 & 0 & -2.18120 & 3.68910 & -1.47180 \\
\hline 32 & 1 & 0 & -1.45100 & 3.94910 & 0.13670 \\
\hline 33 & 7 & 0 & -1.16570 & 1.02670 & 0.32880 \\
\hline 34 & 22 & 0 & -2.65090 & -0.36490 & 0.06050 \\
\hline 35 & 17 & 0 & -1.42360 & -1.97820 & 1.10570 \\
\hline 36 & 17 & 0 & -3.75850 & 0.22010 & 1.93670 \\
\hline 37 & 17 & 0 & -4.39320 & -1.38740 & -0.96000 \\
\hline 38 & 17 & 0 & -1.32410 & -0.80930 & -2.01620 \\
\hline
\end{tabular}




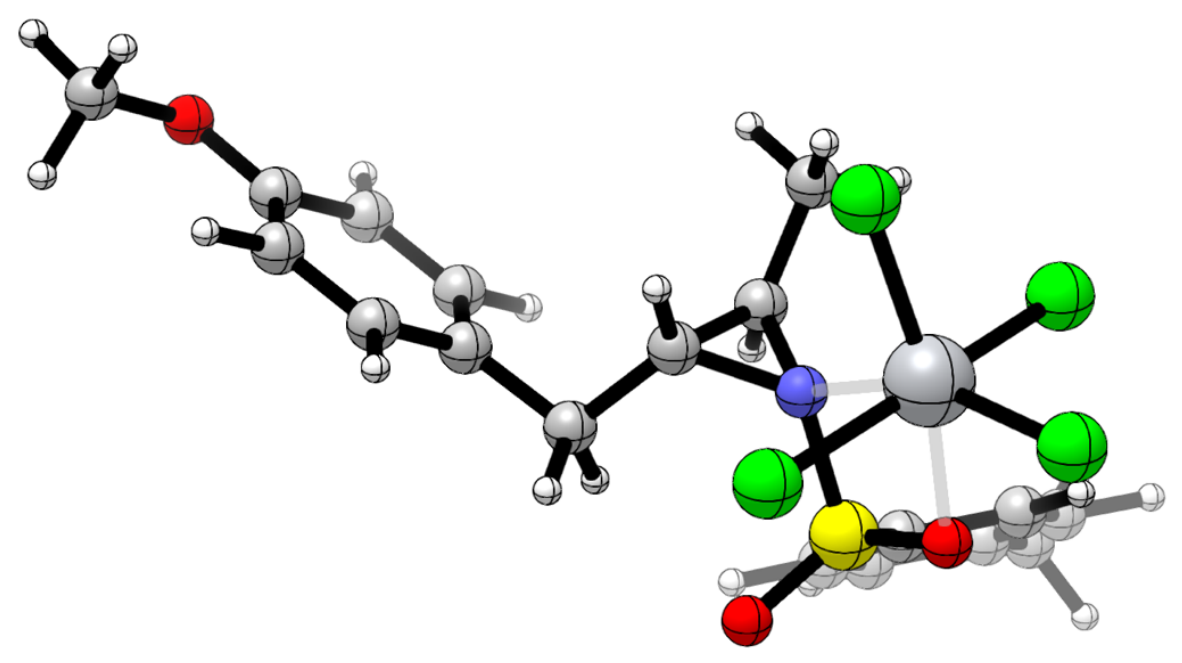

Sum of electronic and thermal free energies: -3273.982526 a.u.

$E_{\text {sPC }}\left[\left(I E F-P C M: \mathrm{CH}_{2} \mathrm{Cl}_{2}, \mathrm{SMD}\right) / \mathrm{MN15} /\right.$ def2-QZVPP] $]=-4067.496284$ a.u.

$\mathrm{E}_{\mathrm{SPC}(\text { corrected) }}\left[\left(\mathrm{IEF}-\mathrm{PCM}: \mathrm{CH}_{2} \mathrm{Cl}_{2}, \mathrm{SMD}\right) / \mathrm{MN} 15 /\right.$ def2-QZVPP, $\left.25^{\circ} \mathrm{C}, 1.0 \mathrm{M}\right]=-4067.182744$ a.u. Number of imaginary frequencies: 0

\begin{tabular}{cccccc}
$\begin{array}{c}\text { Center } \\
\text { Number }\end{array}$ & $\begin{array}{c}\text { Atomic } \\
\text { Number }\end{array}$ & $\begin{array}{c}\text { Atomic } \\
\text { Type }\end{array}$ & \multicolumn{3}{c}{ Coordinates (Ångstroms) } \\
& & & $\mathrm{X}$ & $\mathrm{Y}$ & $\mathrm{Z}$ \\
\hline 1 & 6 & 0 & -5.55200 & -0.56440 & -0.55820 \\
2 & 6 & 0 & -4.23140 & -0.61660 & -1.02210 \\
3 & 6 & 0 & -3.29810 & 0.36450 & -0.68080 \\
4 & 6 & 0 & -3.71330 & 1.41470 & 0.15590 \\
5 & 6 & 0 & -5.01970 & 1.48320 & 0.62710 \\
6 & 6 & 0 & -5.94840 & 0.49090 & 0.27210 \\
7 & 8 & 0 & -7.20360 & 0.63650 & 0.77310 \\
8 & 6 & 0 & -8.17740 & -0.33850 & 0.43620 \\
9 & 6 & 0 & -1.86290 & 0.28400 & -1.15530 \\
10 & 6 & 0 & -1.03770 & -0.32150 & -0.03930 \\
11 & 6 & 0 & -0.37940 & 0.53140 & 0.97370 \\
12 & 7 & 0 & 0.45730 & -0.25300 & 0.00060 \\
13 & 6 & 0 & -0.30830 & 0.21260 & 2.43780 \\
14 & 16 & 0 & 1.41670 & 0.49230 & -1.19790 \\
15 & 8 & 0 & 2.57100 & -0.45240 & -1.09610 \\
16 & 8 & 0 & 0.73250 & 0.60580 & -2.46710 \\
17 & 6 & 0 & 1.92050 & 2.05900 & -0.62710
\end{tabular}




\begin{tabular}{|c|c|c|c|c|c|}
\hline 18 & 6 & 0 & 1.13300 & 3.16410 & -0.97120 \\
\hline 19 & 6 & 0 & 1.52710 & 4.42040 & -0.51970 \\
\hline 20 & 6 & 0 & 2.68980 & 4.57910 & 0.25250 \\
\hline 21 & 6 & 0 & 3.45920 & 3.44630 & 0.57080 \\
\hline 22 & 6 & 0 & 3.08580 & 2.17780 & 0.13910 \\
\hline 23 & 6 & 0 & 3.12560 & 5.94110 & 0.71840 \\
\hline 24 & 1 & 0 & -6.25170 & -1.34240 & -0.85000 \\
\hline 25 & 1 & 0 & -3.92950 & -1.44000 & -1.66890 \\
\hline 26 & 1 & 0 & -3.00160 & 2.19350 & 0.43190 \\
\hline 27 & 1 & 0 & -5.34470 & 2.29960 & 1.26850 \\
\hline 28 & 1 & 0 & -7.88070 & -1.33380 & 0.79280 \\
\hline 29 & 1 & 0 & -9.10050 & -0.03570 & 0.93500 \\
\hline 30 & 1 & 0 & -8.34430 & -0.37280 & -0.64860 \\
\hline 31 & 1 & 0 & -1.78400 & -0.33460 & -2.05660 \\
\hline 32 & 1 & 0 & -1.48740 & 1.28770 & -1.39380 \\
\hline 33 & 1 & 0 & -1.35790 & -1.31670 & 0.27760 \\
\hline 34 & 1 & 0 & -0.39170 & 1.59700 & 0.73040 \\
\hline 35 & 1 & 0 & -0.24300 & -0.86540 & 2.61360 \\
\hline 36 & 1 & 0 & 0.54420 & 0.70970 & 2.91070 \\
\hline 37 & 1 & 0 & -1.23030 & 0.58640 & 2.90090 \\
\hline 38 & 1 & 0 & 0.23940 & 3.04270 & -1.58050 \\
\hline 39 & 1 & 0 & 0.92960 & 5.29310 & -0.77400 \\
\hline 40 & 1 & 0 & 4.36710 & 3.56450 & 1.15890 \\
\hline 41 & 1 & 0 & 3.68810 & 1.30400 & 0.37090 \\
\hline 42 & 1 & 0 & 2.34790 & 6.69070 & 0.54050 \\
\hline 43 & 1 & 0 & 3.36650 & 5.92940 & 1.78800 \\
\hline 44 & 1 & 0 & 4.03210 & 6.25480 & 0.18440 \\
\hline 45 & 22 & 0 & 1.85820 & -2.06160 & 0.16460 \\
\hline 46 & 17 & 0 & 2.81270 & -0.90100 & 1.86470 \\
\hline 47 & 17 & 0 & 3.62650 & -3.29150 & -0.29910 \\
\hline 48 & 17 & 0 & 0.59750 & -3.28670 & 1.51930 \\
\hline 49 & 17 & 0 & 0.65810 & -2.70600 & -1.68060 \\
\hline
\end{tabular}




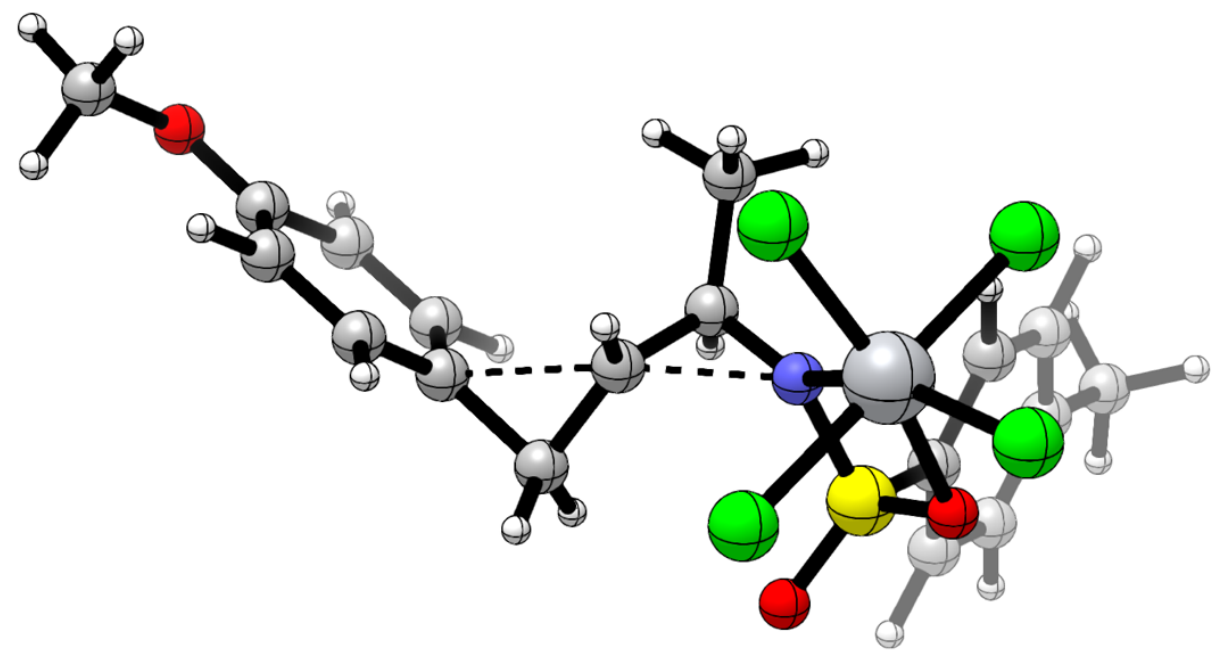

Sum of electronic and thermal free energies: -3273.957796 a.u.

$E_{S P C}\left[\left(I E F-P C M: \mathrm{CH}_{2} \mathrm{Cl}_{2}, \mathrm{SMD}\right) / \mathrm{MN15} /\right.$ def2-QZVPP] $=-4067.471904$ a.u.

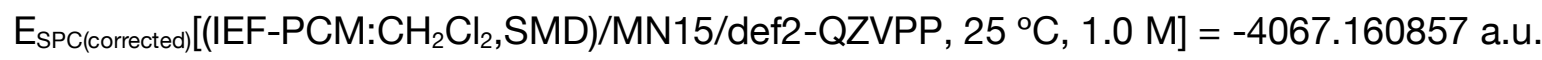
Number of imaginary frequencies: 1

\begin{tabular}{|c|c|c|c|c|c|}
\hline \multirow{2}{*}{$\begin{array}{l}\text { Center } \\
\text { Number }\end{array}$} & \multirow{2}{*}{$\begin{array}{l}\text { Atomic } \\
\text { Number }\end{array}$} & \multirow{2}{*}{$\begin{array}{l}\text { Atomic } \\
\text { Type }\end{array}$} & \multicolumn{3}{|c|}{ Coordinates (Ångstroms) } \\
\hline & & & $X$ & Y & Z \\
\hline 1 & 6 & 0 & -5.44780 & -0.20180 & -0.28730 \\
\hline 2 & 6 & 0 & -4.22380 & -0.46680 & -0.89560 \\
\hline 3 & 6 & 0 & -3.24860 & 0.53600 & -1.02800 \\
\hline 4 & 6 & 0 & -3.50110 & 1.81290 & -0.48050 \\
\hline 5 & 6 & 0 & -4.71460 & 2.08860 & 0.12480 \\
\hline 6 & 6 & 0 & -5.69770 & 1.08340 & 0.22320 \\
\hline 7 & 8 & 0 & -6.84840 & 1.44070 & 0.82470 \\
\hline 8 & 6 & 0 & -7.88410 & 0.47280 & 0.95680 \\
\hline 9 & 6 & 0 & -1.91260 & 0.23990 & -1.71580 \\
\hline 10 & 6 & 0 & -1.39600 & -0.18850 & -0.42050 \\
\hline 11 & 6 & 0 & -0.56180 & 0.64140 & 0.47130 \\
\hline 12 & 7 & 0 & 0.55320 & -0.19730 & -0.01720 \\
\hline 13 & 6 & 0 & -0.77410 & 0.58230 & 1.96580 \\
\hline 14 & 16 & 0 & 1.60920 & 0.36370 & -1.11850 \\
\hline 15 & 8 & 0 & 2.57000 & -0.79830 & -0.98790 \\
\hline 16 & 8 & 0 & 1.09910 & 0.62410 & -2.45360 \\
\hline 17 & 6 & 0 & 2.33010 & 1.84310 & -0.50900 \\
\hline
\end{tabular}




\begin{tabular}{|c|c|c|c|c|c|}
\hline 18 & 6 & 0 & 2.62750 & 2.85970 & -1.41990 \\
\hline 19 & 6 & 0 & 3.21820 & 4.02910 & -0.94190 \\
\hline 20 & 6 & 0 & 3.50610 & 4.19090 & 0.42100 \\
\hline 21 & 6 & 0 & 3.18930 & 3.14830 & 1.31080 \\
\hline 22 & 6 & 0 & 2.60270 & 1.97200 & 0.85850 \\
\hline 23 & 6 & 0 & 4.13980 & 5.45490 & 0.93530 \\
\hline 24 & 1 & 0 & -6.19150 & -0.98890 & -0.21210 \\
\hline 25 & 1 & 0 & -4.02200 & -1.46160 & -1.29060 \\
\hline 26 & 1 & 0 & -2.74080 & 2.58910 & -0.55770 \\
\hline 27 & 1 & 0 & -4.93160 & 3.07300 & 0.53200 \\
\hline 28 & 1 & 0 & -7.54930 & -0.37760 & 1.56350 \\
\hline 29 & 1 & 0 & -8.70890 & 0.97890 & 1.46120 \\
\hline 30 & 1 & 0 & -8.21700 & 0.12000 & -0.02710 \\
\hline 31 & 1 & 0 & -1.98950 & -0.55610 & -2.45870 \\
\hline 32 & 1 & 0 & -1.44870 & 1.14430 & -2.11330 \\
\hline 33 & 1 & 0 & -1.64780 & -1.18860 & -0.06540 \\
\hline 34 & 1 & 0 & -0.46030 & 1.67350 & 0.10740 \\
\hline 35 & 1 & 0 & -0.86730 & -0.45320 & 2.30920 \\
\hline 36 & 1 & 0 & 0.07230 & 1.05330 & 2.47800 \\
\hline 37 & 1 & 0 & -1.69150 & 1.12660 & 2.22020 \\
\hline 38 & 1 & 0 & 2.39820 & 2.73970 & -2.47530 \\
\hline 39 & 1 & 0 & 3.45700 & 4.83040 & -1.63820 \\
\hline 40 & 1 & 0 & 3.40470 & 3.26580 & 2.37120 \\
\hline 41 & 1 & 0 & 2.35880 & 1.16830 & 1.54900 \\
\hline 42 & 1 & 0 & 3.48840 & 5.94400 & 1.67060 \\
\hline 43 & 1 & 0 & 5.08920 & 5.23510 & 1.43950 \\
\hline 44 & 1 & 0 & 4.33480 & 6.16160 & 0.12200 \\
\hline 45 & 22 & 0 & 1.45150 & -2.12220 & 0.22290 \\
\hline 46 & 17 & 0 & 2.48590 & -1.35150 & 2.12560 \\
\hline 47 & 17 & 0 & 3.02260 & -3.69500 & -0.07210 \\
\hline 48 & 17 & 0 & -0.20940 & -3.03840 & 1.43480 \\
\hline 49 & 17 & 0 & 0.36180 & -2.77100 & -1.74430 \\
\hline
\end{tabular}




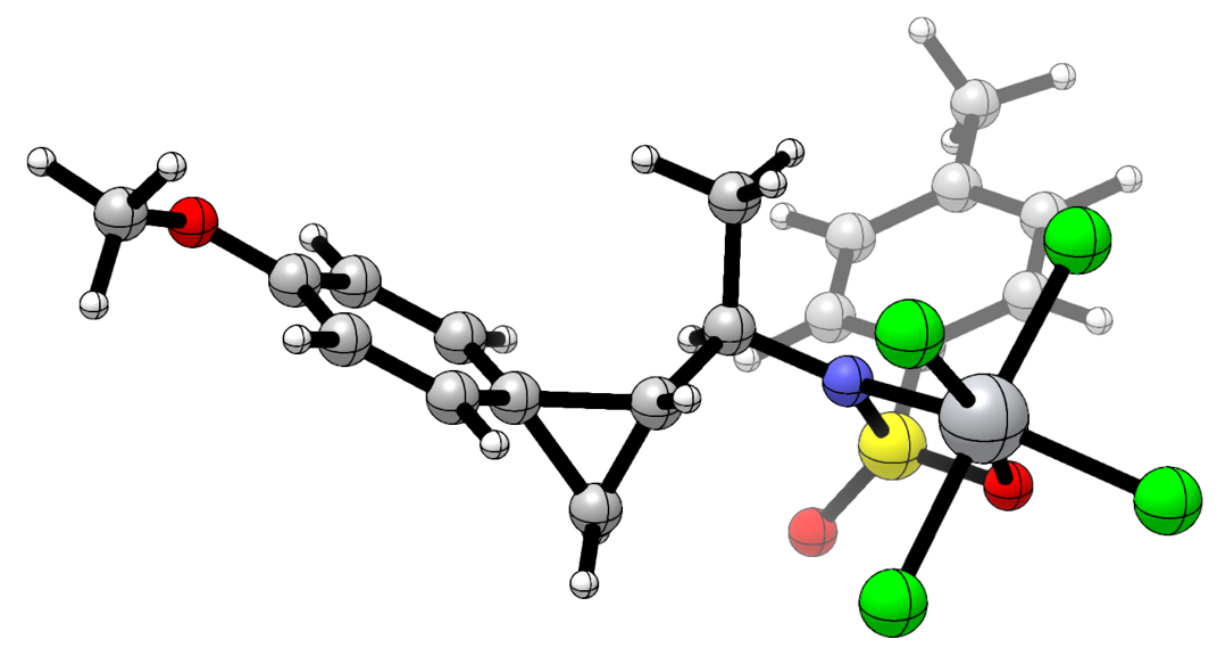

Sum of electronic and thermal free energies: -3273.973327 a.u.

$\mathrm{E}_{\mathrm{SPC}}\left[\left(\mathrm{IEF}-\mathrm{PCM}: \mathrm{CH}_{2} \mathrm{Cl}_{2}, \mathrm{SMD}\right) / \mathrm{MN15} /\right.$ def2-QZVPP] $=-4067.488230$ a.u.

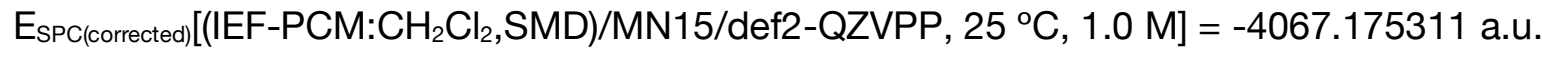

Number of imaginary frequencies: 0

\begin{tabular}{|c|c|c|c|c|c|}
\hline \multirow{2}{*}{$\begin{array}{l}\text { Center } \\
\text { Number }\end{array}$} & \multirow{2}{*}{$\begin{array}{l}\text { Atomic } \\
\text { Number }\end{array}$} & \multirow{2}{*}{$\begin{array}{l}\text { Atomic } \\
\text { Type }\end{array}$} & \multicolumn{3}{|c|}{ Coordinates (Ångstroms) } \\
\hline & & & $\mathrm{X}$ & Y & $\mathrm{Z}$ \\
\hline 1 & 6 & 0 & -5.52960 & -0.42840 & 0.15240 \\
\hline 2 & 6 & 0 & -4.31320 & -0.89420 & -0.27590 \\
\hline 3 & 6 & 0 & -3.25330 & -0.00450 & -0.66040 \\
\hline 4 & 6 & 0 & -3.50700 & 1.41240 & -0.60100 \\
\hline 5 & 6 & 0 & -4.71640 & 1.88790 & -0.18160 \\
\hline 6 & 6 & 0 & -5.73830 & 0.97530 & 0.20680 \\
\hline 7 & 8 & 0 & -6.86650 & 1.52630 & 0.60660 \\
\hline 8 & 6 & 0 & -7.97190 & 0.70960 & 1.02090 \\
\hline 9 & 6 & 0 & -2.23420 & -0.53010 & -1.75460 \\
\hline 10 & 6 & 0 & -1.78670 & -0.56570 & -0.38310 \\
\hline 11 & 6 & 0 & -0.71980 & 0.38150 & 0.14220 \\
\hline 12 & 7 & 0 & 0.57330 & -0.25000 & -0.14210 \\
\hline 13 & 6 & 0 & -0.85580 & 0.69850 & 1.62560 \\
\hline 14 & 16 & 0 & 1.56210 & 0.39070 & -1.23090 \\
\hline 15 & 8 & 0 & 2.60680 & -0.69310 & -1.15750 \\
\hline 16 & 8 & 0 & 0.94070 & 0.68420 & -2.51470 \\
\hline 17 & 6 & 0 & 2.27770 & 1.87700 & -0.62730 \\
\hline 18 & 6 & 0 & 1.60480 & 3.08270 & -0.86660 \\
\hline
\end{tabular}




\begin{tabular}{|c|c|c|c|c|c|}
\hline 19 & 6 & 0 & 2.14150 & 4.25970 & -0.35500 \\
\hline 20 & 6 & 0 & 3.34150 & 4.25010 & 0.37840 \\
\hline 21 & 6 & 0 & 3.99710 & 3.02820 & 0.58710 \\
\hline 22 & 6 & 0 & 3.47280 & 1.83300 & 0.09330 \\
\hline 23 & 6 & 0 & 3.90750 & 5.53660 & 0.91810 \\
\hline 24 & 1 & 0 & -6.31310 & -1.12000 & 0.44370 \\
\hline 25 & 1 & 0 & -4.12490 & -1.96550 & -0.32670 \\
\hline 26 & 1 & 0 & -2.73000 & 2.10680 & -0.91300 \\
\hline 27 & 1 & 0 & -4.93100 & 2.95170 & -0.13570 \\
\hline 28 & 1 & 0 & -7.69000 & 0.10370 & 1.88780 \\
\hline 29 & 1 & 0 & -8.76450 & 1.40610 & 1.29410 \\
\hline 30 & 1 & 0 & -8.30140 & 0.07170 & 0.19480 \\
\hline 31 & 1 & 0 & -2.55110 & -1.44570 & -2.24610 \\
\hline 32 & 1 & 0 & -1.86950 & 0.26630 & -2.40110 \\
\hline 33 & 1 & 0 & -1.82790 & -1.52370 & 0.13430 \\
\hline 34 & 1 & 0 & -0.76630 & 1.31840 & -0.43350 \\
\hline 35 & 1 & 0 & -0.81540 & -0.21890 & 2.22420 \\
\hline 36 & 1 & 0 & -0.03540 & 1.35600 & 1.93900 \\
\hline 37 & 1 & 0 & -1.80940 & 1.20600 & 1.81980 \\
\hline 38 & 1 & 0 & 0.68880 & 3.09760 & -1.45350 \\
\hline 39 & 1 & 0 & 1.63160 & 5.20470 & -0.53430 \\
\hline 40 & 1 & 0 & 4.93390 & 3.01210 & 1.14050 \\
\hline 41 & 1 & 0 & 3.98340 & 0.88760 & 0.25280 \\
\hline 42 & 1 & 0 & 4.86800 & 5.36950 & 1.41640 \\
\hline 43 & 1 & 0 & 4.05680 & 6.26600 & 0.11210 \\
\hline 44 & 1 & 0 & 3.21850 & 5.99010 & 1.64220 \\
\hline 45 & 22 & 0 & 1.56680 & -1.94400 & 0.25570 \\
\hline 46 & 17 & 0 & 2.67110 & -0.92920 & 2.04620 \\
\hline 47 & 17 & 0 & 3.32150 & -3.40350 & 0.03160 \\
\hline 48 & 17 & 0 & 0.06250 & -2.86290 & 1.66640 \\
\hline 49 & 17 & 0 & 0.50510 & -2.99680 & -1.56690 \\
\hline
\end{tabular}




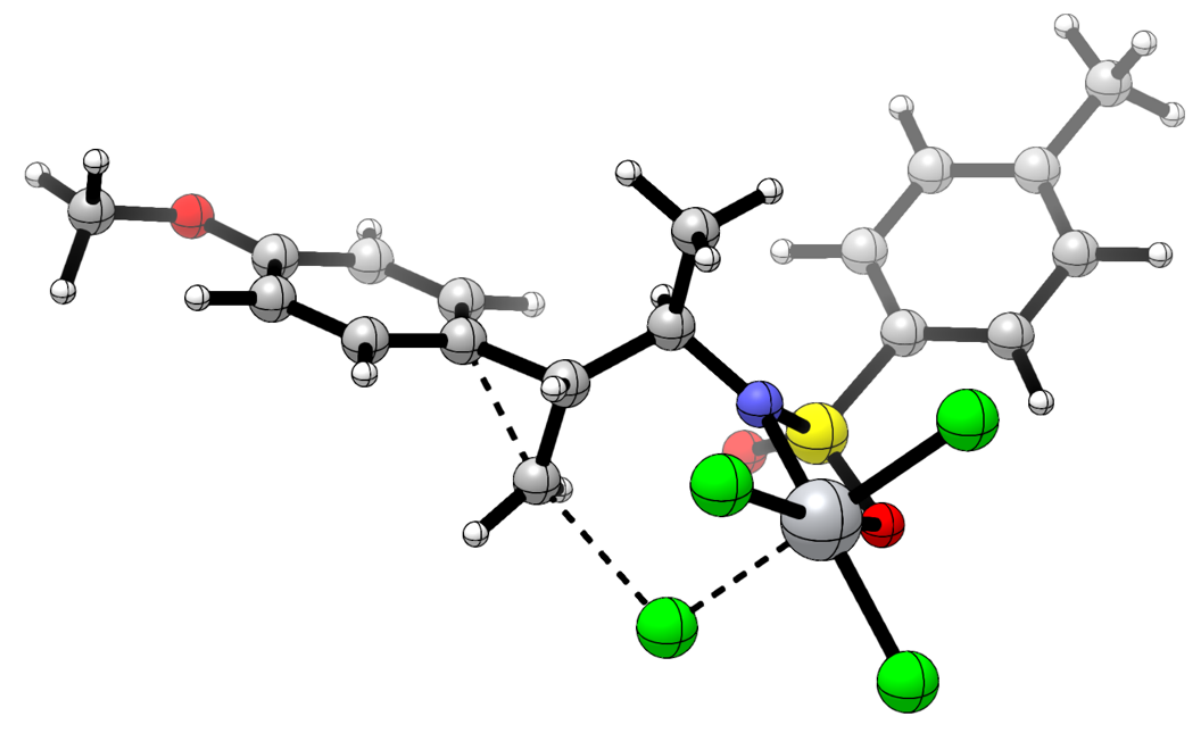

Sum of electronic and thermal free energies: -3273.959178 a.u.

$E_{S P C}\left[\left(I E F-P C M: \mathrm{CH}_{2} \mathrm{Cl}_{2}, \mathrm{SMD}\right) / \mathrm{MN} 15 /\right.$ def2-QZVPP] $=-4067.474194$ a.u.

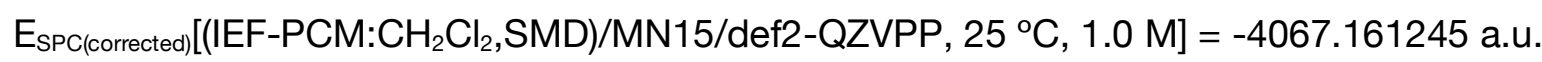

Number of imaginary frequencies: 1

\begin{tabular}{|c|c|c|c|c|c|}
\hline \multirow{2}{*}{$\begin{array}{l}\text { Center } \\
\text { Number }\end{array}$} & \multirow{2}{*}{$\begin{array}{l}\text { Atomic } \\
\text { Number }\end{array}$} & \multirow{2}{*}{$\begin{array}{c}\text { Atomic } \\
\text { Type }\end{array}$} & \multicolumn{3}{|c|}{ Coordinates (Ångstroms) } \\
\hline & & & $\mathrm{X}$ & $\mathrm{Y}$ & $\mathrm{Z}$ \\
\hline 1 & 6 & 0 & -5.58530 & -0.19290 & 0.61820 \\
\hline 2 & 6 & 0 & -4.30880 & -0.72190 & 0.72640 \\
\hline 3 & 6 & 0 & -3.18440 & -0.03990 & 0.21030 \\
\hline 4 & 6 & 0 & -3.39090 & 1.17770 & -0.48330 \\
\hline 5 & 6 & 0 & -4.66060 & 1.71120 & -0.60120 \\
\hline 6 & 6 & 0 & -5.76600 & 1.03740 & -0.04540 \\
\hline 7 & 8 & 0 & -6.95770 & 1.63210 & -0.20130 \\
\hline 8 & 6 & 0 & -8.12340 & 1.00240 & 0.32560 \\
\hline 9 & 6 & 0 & -2.00470 & -1.17200 & -0.90470 \\
\hline 10 & 6 & 0 & -1.78810 & -0.65050 & 0.43240 \\
\hline 11 & 6 & 0 & -0.63540 & 0.33340 & 0.66900 \\
\hline 12 & 7 & 0 & 0.60290 & -0.22090 & 0.11550 \\
\hline 13 & 6 & 0 & -0.46920 & 0.66090 & 2.14690 \\
\hline 14 & 16 & 0 & 1.31160 & 0.49860 & -1.13800 \\
\hline 15 & 8 & 0 & 2.31680 & -0.59570 & -1.39690 \\
\hline 16 & 8 & 0 & 0.39580 & 0.88290 & -2.20200 \\
\hline 17 & 6 & 0 & 2.18360 & 1.92640 & -0.61410 \\
\hline
\end{tabular}




\begin{tabular}{|c|c|c|c|c|c|}
\hline 18 & 6 & 0 & 1.49150 & 3.14080 & -0.56580 \\
\hline 19 & 6 & 0 & 2.16160 & 4.27180 & -0.10270 \\
\hline 20 & 6 & 0 & 3.50590 & 4.20330 & 0.29520 \\
\hline 21 & 6 & 0 & 4.17440 & 2.96940 & 0.22040 \\
\hline 22 & 6 & 0 & 3.52420 & 1.82290 & -0.22720 \\
\hline 23 & 6 & 0 & 4.23180 & 5.42730 & 0.78560 \\
\hline 24 & 1 & 0 & -6.42800 & -0.73000 & 1.04210 \\
\hline 25 & 1 & 0 & -4.16080 & -1.67350 & 1.23440 \\
\hline 26 & 1 & 0 & -2.55310 & 1.70080 & -0.94010 \\
\hline 27 & 1 & 0 & -4.82960 & 2.64770 & -1.12640 \\
\hline 28 & 1 & 0 & -8.04510 & 0.88720 & 1.41320 \\
\hline 29 & 1 & 0 & -8.95700 & 1.66430 & 0.08620 \\
\hline 30 & 1 & 0 & -8.28290 & 0.02460 & -0.14450 \\
\hline 31 & 1 & 0 & -2.62030 & -2.05300 & -1.05050 \\
\hline 32 & 1 & 0 & -1.77470 & -0.55260 & -1.77020 \\
\hline 33 & 1 & 0 & -1.84820 & -1.41450 & 1.21140 \\
\hline 34 & 1 & 0 & -0.84890 & 1.26130 & 0.12040 \\
\hline 35 & 1 & 0 & -0.21950 & -0.24390 & 2.71480 \\
\hline 36 & 1 & 0 & 0.33750 & 1.39240 & 2.27770 \\
\hline 37 & 1 & 0 & -1.39890 & 1.08360 & 2.54780 \\
\hline 38 & 1 & 0 & 0.45500 & 3.20190 & -0.89150 \\
\hline 39 & 1 & 0 & 1.63820 & 5.22480 & -0.05820 \\
\hline 40 & 1 & 0 & 5.22090 & 2.90980 & 0.51390 \\
\hline 41 & 1 & 0 & 4.04320 & 0.87060 & -0.28980 \\
\hline 42 & 1 & 0 & 4.60590 & 5.27430 & 1.80570 \\
\hline 43 & 1 & 0 & 5.10040 & 5.64420 & 0.15120 \\
\hline 44 & 1 & 0 & 3.57670 & 6.30470 & 0.78560 \\
\hline 45 & 22 & 0 & 1.62910 & -1.91760 & 0.14090 \\
\hline 46 & 17 & 0 & 3.22120 & -1.17750 & 1.58750 \\
\hline 47 & 17 & 0 & 2.99650 & -3.49030 & -0.76710 \\
\hline 48 & 17 & 0 & 0.43900 & -2.93080 & 1.74070 \\
\hline 49 & 17 & 0 & -0.08220 & -2.66440 & -1.51670 \\
\hline
\end{tabular}




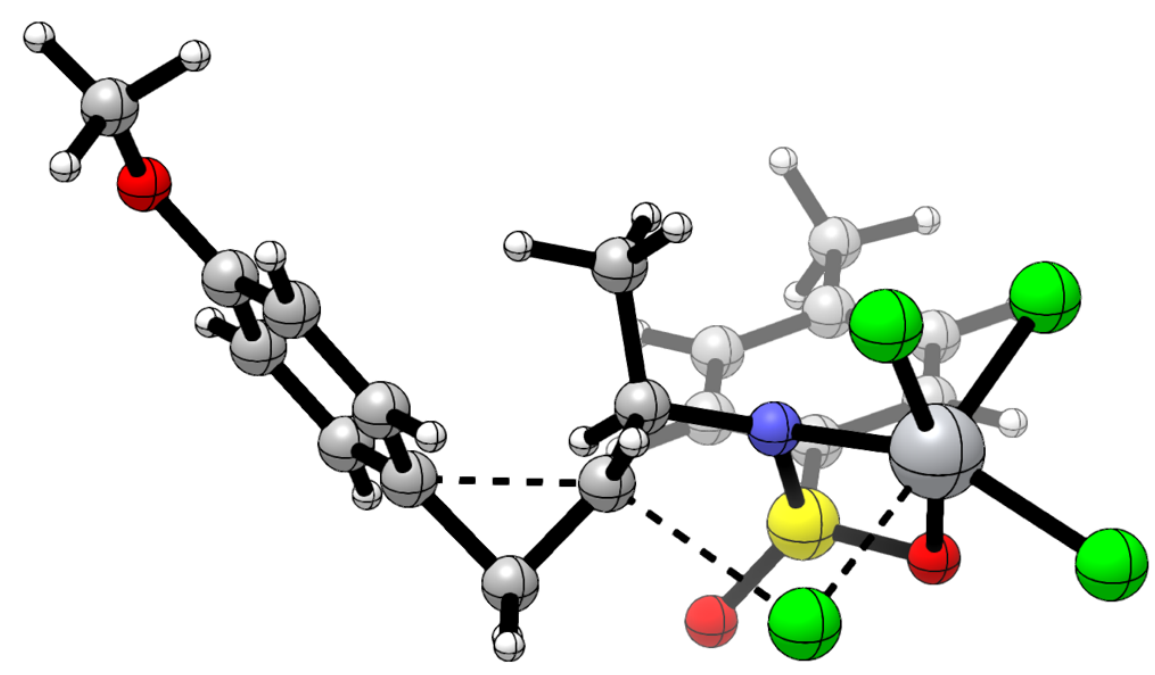

Sum of electronic and thermal free energies: -3273.954132 a.u.

$E_{\text {sPC }}\left[\left(I E F-P C M: \mathrm{CH}_{2} \mathrm{Cl}_{2}, \mathrm{SMD}\right) / \mathrm{MN15} /\right.$ def2-QZVPP] $=-4067.469941$ a.u.

$\mathrm{E}_{\mathrm{SPC}(\text { corrected) }}\left[\left(\mathrm{IEF}-\mathrm{PCM}: \mathrm{CH}_{2} \mathrm{Cl}_{2}, \mathrm{SMD}\right) / \mathrm{MN} 15 /\right.$ def2-QZVPP, $\left.25^{\circ} \mathrm{C}, 1.0 \mathrm{M}\right]=-4067.156806$ a.u. Number of imaginary frequencies: 1

\begin{tabular}{cccccc}
$\begin{array}{c}\text { Center } \\
\text { Number }\end{array}$ & $\begin{array}{c}\text { Atomic } \\
\text { Number }\end{array}$ & $\begin{array}{c}\text { Atomic } \\
\text { Type }\end{array}$ & \multicolumn{3}{c}{ Coordinates (Angstroms) } \\
& & & $\mathrm{X}$ & $\mathrm{Y}$ & $\mathrm{Z}$ \\
\hdashline 1 & 6 & 0 & -5.32470 & -0.27200 & 0.36130 \\
2 & 6 & 0 & -4.34700 & -0.93290 & -0.37830 \\
3 & 6 & 0 & -3.46980 & -0.22680 & -1.22350 \\
4 & 6 & 0 & -3.58320 & 1.18200 & -1.30230 \\
5 & 6 & 0 & -4.55660 & 1.84600 & -0.58410 \\
6 & 6 & 0 & -5.42810 & 1.12480 & 0.26410 \\
7 & 8 & 0 & -6.32850 & 1.85950 & 0.93690 \\
8 & 6 & 0 & -7.23790 & 1.20290 & 1.81570 \\
9 & 6 & 0 & -2.36860 & -0.96220 & -1.98350 \\
10 & 6 & 0 & -1.63970 & -1.05760 & -0.72390 \\
11 & 6 & 0 & -0.85670 & 0.10990 & -0.11800 \\
12 & 7 & 0 & 0.56710 & -0.22020 & -0.03590 \\
13 & 6 & 0 & -1.38740 & 0.47990 & 1.26740 \\
14 & 16 & 0 & 1.56340 & 0.38610 & -1.15450 \\
15 & 8 & 0 & 2.61730 & -0.68630 & -1.03290 \\
16 & 8 & 0 & 0.93650 & 0.62310 & -2.44510 \\
17 & 6 & 0 & 2.27020 & 1.88740 & -0.58810
\end{tabular}




\begin{tabular}{|c|c|c|c|c|c|}
\hline 18 & 6 & 0 & 1.55260 & 3.07230 & -0.79920 \\
\hline 19 & 6 & 0 & 2.08210 & 4.26410 & -0.31710 \\
\hline 20 & 6 & 0 & 3.31710 & 4.28890 & 0.35690 \\
\hline 21 & 6 & 0 & 4.01390 & 3.08610 & 0.53870 \\
\hline 22 & 6 & 0 & 3.49920 & 1.87550 & 0.07280 \\
\hline 23 & 6 & 0 & 3.87400 & 5.59170 & 0.86510 \\
\hline 24 & 1 & 0 & -5.99060 & -0.84120 & 1.00220 \\
\hline 25 & 1 & 0 & -4.26320 & -2.01640 & -0.30740 \\
\hline 26 & 1 & 0 & -2.90770 & 1.73820 & -1.95020 \\
\hline 27 & 1 & 0 & -4.67090 & 2.92520 & -0.65030 \\
\hline 28 & 1 & 0 & -6.69800 & 0.67920 & 2.61390 \\
\hline 29 & 1 & 0 & -7.86000 & 1.98810 & 2.24820 \\
\hline 30 & 1 & 0 & -7.86900 & 0.49520 & 1.26470 \\
\hline 31 & 1 & 0 & -2.68610 & -1.92170 & -2.39350 \\
\hline 32 & 1 & 0 & -1.89220 & -0.31760 & -2.72690 \\
\hline 33 & 1 & 0 & -1.90220 & -1.86820 & -0.04320 \\
\hline 34 & 1 & 0 & -0.96820 & 0.96850 & -0.79580 \\
\hline 35 & 1 & 0 & -1.29910 & -0.36770 & 1.95560 \\
\hline 36 & 1 & 0 & -0.79220 & 1.31450 & 1.65620 \\
\hline 37 & 1 & 0 & -2.43920 & 0.78600 & 1.21740 \\
\hline 38 & 1 & 0 & 0.60500 & 3.05940 & -1.33410 \\
\hline 39 & 1 & 0 & 1.53870 & 5.19450 & -0.47190 \\
\hline 40 & 1 & 0 & 4.97540 & 3.09660 & 1.04800 \\
\hline 41 & 1 & 0 & 4.04230 & 0.94480 & 0.20990 \\
\hline 42 & 1 & 0 & 3.19320 & 6.04610 & 1.59600 \\
\hline 43 & 1 & 0 & 4.84750 & 5.44670 & 1.34490 \\
\hline 44 & 1 & 0 & 3.99550 & 6.31010 & 0.04460 \\
\hline 45 & 22 & 0 & 1.59310 & -1.89270 & 0.40450 \\
\hline 46 & 17 & 0 & 2.85680 & -0.99970 & 2.05250 \\
\hline 47 & 17 & 0 & 3.03480 & -3.55510 & -0.09430 \\
\hline 48 & 17 & 0 & 0.09540 & -2.76240 & 1.82650 \\
\hline 49 & 17 & 0 & 0.17480 & -2.70570 & -1.46210 \\
\hline
\end{tabular}




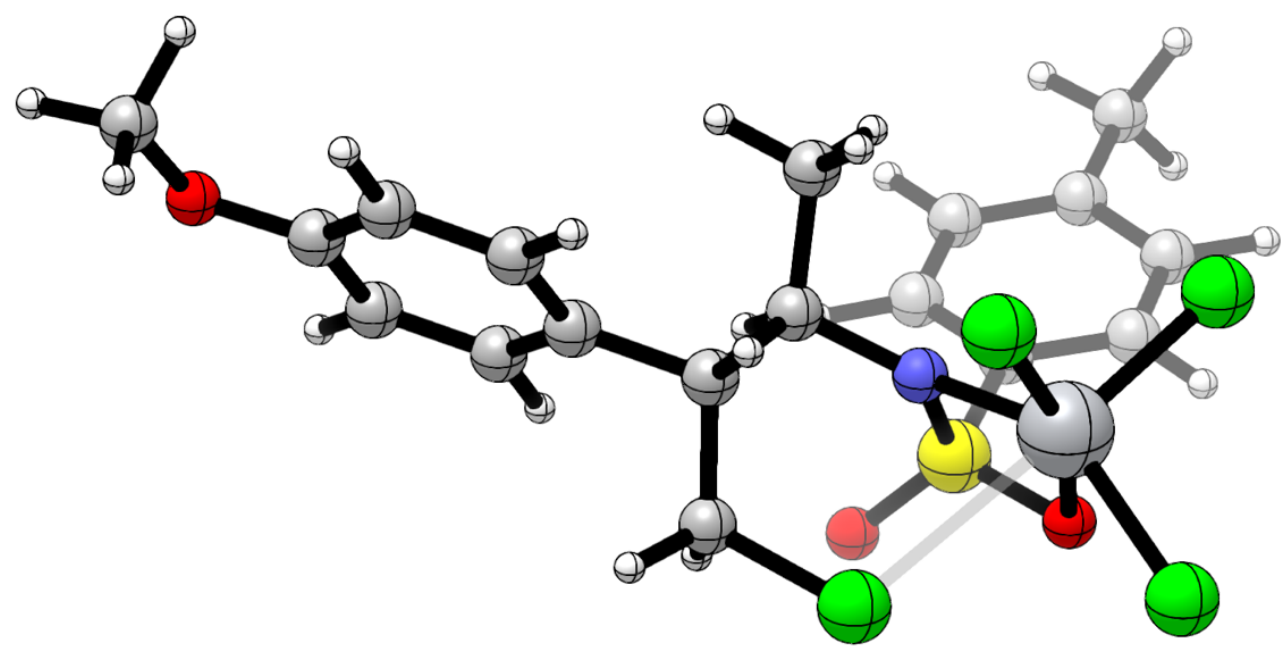

Sum of electronic and thermal free energies: -3273.986559 a.u.

$E_{s P C}\left[\left(I E F-P C M: \mathrm{CH}_{2} \mathrm{Cl}_{2}, \mathrm{SMD}\right) / \mathrm{MN15} / \mathrm{def2}-\mathrm{QZVPP}\right]=-4067.504362$ a.u.

$E_{\text {sPC(corrected) }}$ [(IEF-PCM: $\left.\mathrm{CH}_{2} \mathrm{Cl}_{2}, \mathrm{SMD}\right) / \mathrm{MN} 15 /$ def2-QZVPP, $\left.25^{\circ} \mathrm{C}, 1.0 \mathrm{M}\right]=-4067.189532$ a.u. Number of imaginary frequencies: 0

\begin{tabular}{|c|c|c|c|c|c|}
\hline \multirow{2}{*}{$\begin{array}{l}\text { Center } \\
\text { Number }\end{array}$} & \multirow{2}{*}{$\begin{array}{l}\text { Atomic } \\
\text { Number }\end{array}$} & \multirow{2}{*}{$\begin{array}{l}\text { Atomic } \\
\text { Type }\end{array}$} & \multicolumn{3}{|c|}{ Coordinates (Ångstroms) } \\
\hline & & & $\mathrm{X}$ & $\mathrm{Y}$ & $\mathrm{Z}$ \\
\hline 1 & 6 & 0 & -5.43760 & 0.03810 & 0.90680 \\
\hline 2 & 6 & 0 & -4.16250 & -0.53810 & 0.85490 \\
\hline 3 & 6 & 0 & -3.20260 & -0.11070 & -0.06530 \\
\hline 4 & 6 & 0 & -3.54830 & 0.92520 & -0.95210 \\
\hline 5 & 6 & 0 & -4.81120 & 1.50570 & -0.91810 \\
\hline 6 & 6 & 0 & -5.76510 & 1.06590 & 0.01420 \\
\hline 7 & 8 & 0 & -6.97150 & 1.68960 & -0.02110 \\
\hline 8 & 6 & 0 & -7.97030 & 1.27270 & 0.89640 \\
\hline 9 & 6 & 0 & -1.61920 & -1.34290 & -1.50600 \\
\hline 10 & 6 & 0 & -1.82710 & -0.75400 & -0.10790 \\
\hline 11 & 6 & 0 & -0.74840 & 0.28370 & 0.28060 \\
\hline 12 & 7 & 0 & 0.60370 & -0.23440 & 0.00240 \\
\hline 13 & 6 & 0 & -0.84170 & 0.73070 & 1.73400 \\
\hline 14 & 16 & 0 & 1.49530 & 0.41450 & -1.17790 \\
\hline 15 & 8 & 0 & 2.50720 & -0.71210 & -1.21940 \\
\hline 16 & 8 & 0 & 0.76170 & 0.72820 & -2.39100 \\
\hline 17 & 6 & 0 & 2.31220 & 1.85580 & -0.61230 \\
\hline 18 & 6 & 0 & 1.60440 & 3.06180 & -0.64450 \\
\hline
\end{tabular}




\begin{tabular}{|c|c|c|c|c|c|}
\hline 19 & 6 & 0 & 2.22900 & 4.21030 & -0.16310 \\
\hline 20 & 6 & 0 & 3.54360 & 4.16760 & 0.32810 \\
\hline 21 & 6 & 0 & 4.22890 & 2.94100 & 0.33040 \\
\hline 22 & 6 & 0 & 3.62360 & 1.77590 & -0.13230 \\
\hline 23 & 6 & 0 & 4.22120 & 5.41220 & 0.83460 \\
\hline 24 & 1 & 0 & -6.15590 & -0.32030 & 1.63860 \\
\hline 25 & 1 & 0 & -3.91450 & -1.33830 & 1.55170 \\
\hline 26 & 1 & 0 & -2.82200 & 1.28300 & -1.68260 \\
\hline 27 & 1 & 0 & -5.08000 & 2.30470 & -1.60570 \\
\hline 28 & 1 & 0 & -7.64410 & 1.42670 & 1.93350 \\
\hline 29 & 1 & 0 & -8.84760 & 1.89150 & 0.69710 \\
\hline 30 & 1 & 0 & -8.22850 & 0.21600 & 0.74670 \\
\hline 31 & 1 & 0 & -2.51250 & -1.87790 & -1.83380 \\
\hline 32 & 1 & 0 & -1.35660 & -0.57780 & -2.24210 \\
\hline 33 & 1 & 0 & -1.81460 & -1.56970 & 0.62620 \\
\hline 34 & 1 & 0 & -0.89350 & 1.15740 & -0.37610 \\
\hline 35 & 1 & 0 & -0.73500 & -0.12700 & 2.41020 \\
\hline 36 & 1 & 0 & -0.04480 & 1.45210 & 1.95380 \\
\hline 37 & 1 & 0 & -1.80930 & 1.20830 & 1.92690 \\
\hline 38 & 1 & 0 & 0.59180 & 3.10400 & -1.04130 \\
\hline 39 & 1 & 0 & 1.69320 & 5.15720 & -0.17700 \\
\hline 40 & 1 & 0 & 5.25300 & 2.90110 & 0.69670 \\
\hline 41 & 1 & 0 & 4.15860 & 0.83040 & -0.13860 \\
\hline 42 & 1 & 0 & 4.56130 & 5.27630 & 1.86880 \\
\hline 43 & 1 & 0 & 5.10720 & 5.64430 & 0.22990 \\
\hline 44 & 1 & 0 & 3.54650 & 6.27380 & 0.80170 \\
\hline 45 & 22 & 0 & 1.69080 & -1.83520 & 0.36040 \\
\hline 46 & 17 & 0 & 3.16650 & -1.12120 & 1.82860 \\
\hline 47 & 17 & 0 & 2.74210 & -3.60670 & -0.52570 \\
\hline 48 & 17 & 0 & 0.28210 & -2.77230 & 1.79140 \\
\hline 49 & 17 & 0 & -0.29370 & -2.59300 & -1.59440 \\
\hline
\end{tabular}




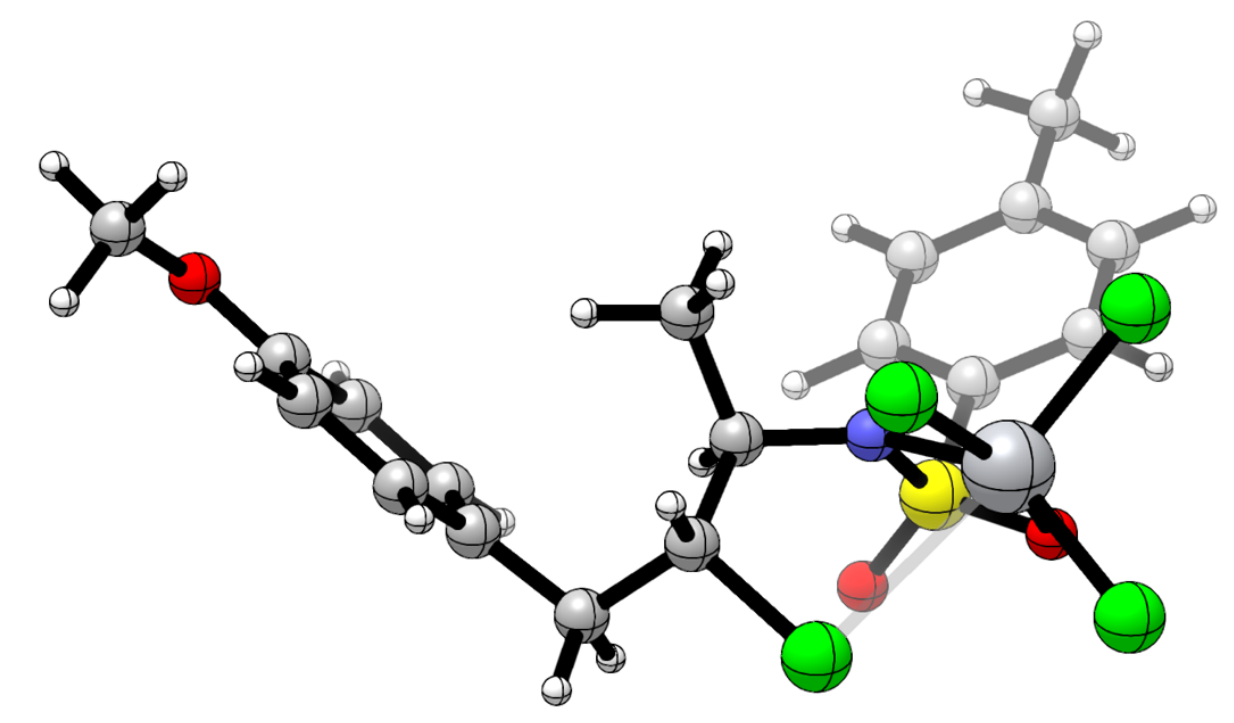

Sum of electronic and thermal free energies: -3273.983385 a.u.

$E_{\text {SPC }}\left[\left(I E F-P C M: \mathrm{CH}_{2} \mathrm{Cl}_{2}, \mathrm{SMD}\right) / \mathrm{MN15} /\right.$ def2-QZVPP] $=-4067.500657$ a.u.

$\mathrm{E}_{\mathrm{SPC}(\text { corrected) }}\left[\left(\mathrm{IEF}-\mathrm{PCM}: \mathrm{CH}_{2} \mathrm{Cl}_{2}, \mathrm{SMD}\right) / \mathrm{MN} 15 /\right.$ def2-QZVPP, $\left.25^{\circ} \mathrm{C}, 1.0 \mathrm{M}\right]=-4067.185763$ a.u.

Number of imaginary frequencies: 0

\begin{tabular}{|c|c|c|c|c|c|}
\hline \multirow{2}{*}{$\begin{array}{l}\text { Center } \\
\text { Number }\end{array}$} & \multirow{2}{*}{$\begin{array}{l}\text { Atomic } \\
\text { Number }\end{array}$} & \multirow{2}{*}{$\begin{array}{c}\text { Atomic } \\
\text { Type }\end{array}$} & \multicolumn{3}{|c|}{ Coordinates (Ångstroms) } \\
\hline & & & $X$ & $\mathrm{Y}$ & $\mathrm{Z}$ \\
\hline 1 & 6 & 0 & -5.52040 & -0.32720 & 0.50600 \\
\hline 2 & 6 & 0 & -4.52460 & -1.07350 & -0.13290 \\
\hline 3 & 6 & 0 & -3.63610 & -0.48600 & -1.03910 \\
\hline 4 & 6 & 0 & -3.77530 & 0.88430 & -1.30970 \\
\hline 5 & 6 & 0 & -4.76040 & 1.64400 & -0.68530 \\
\hline 6 & 6 & 0 & -5.63530 & 1.04310 & 0.23300 \\
\hline 7 & 8 & 0 & -6.56430 & 1.85630 & 0.80070 \\
\hline 8 & 6 & 0 & -7.47400 & 1.29050 & 1.73100 \\
\hline 9 & 6 & 0 & -2.50380 & -1.28860 & -1.63570 \\
\hline 10 & 6 & 0 & -1.39150 & -1.51650 & -0.60680 \\
\hline 11 & 6 & 0 & -0.77570 & -0.18810 & -0.07070 \\
\hline 12 & 7 & 0 & 0.68710 & -0.27410 & 0.02650 \\
\hline 13 & 6 & 0 & -1.36630 & 0.22240 & 1.27580 \\
\hline 14 & 16 & 0 & 1.57540 & 0.42110 & -1.13950 \\
\hline 15 & 8 & 0 & 2.71890 & -0.57130 & -1.06020 \\
\hline 16 & 8 & 0 & 0.88530 & 0.58460 & -2.40520 \\
\hline
\end{tabular}




\begin{tabular}{|c|c|c|c|c|c|}
\hline 17 & 6 & 0 & 2.18670 & 1.97090 & -0.60450 \\
\hline 18 & 6 & 0 & 1.35000 & 3.08230 & -0.74740 \\
\hline 19 & 6 & 0 & 1.80780 & 4.31840 & -0.29770 \\
\hline 20 & 6 & 0 & 3.08400 & 4.45390 & 0.27230 \\
\hline 21 & 6 & 0 & 3.90170 & 3.31720 & 0.38720 \\
\hline 22 & 6 & 0 & 3.46450 & 2.06800 & -0.04490 \\
\hline 23 & 6 & 0 & 3.58170 & 5.79320 & 0.74510 \\
\hline 24 & 1 & 0 & -6.19250 & -0.81760 & 1.20450 \\
\hline 25 & 1 & 0 & -4.43550 & -2.13690 & 0.09070 \\
\hline 26 & 1 & 0 & -3.09660 & 1.36170 & -2.01660 \\
\hline 27 & 1 & 0 & -4.86940 & 2.70590 & -0.89520 \\
\hline 28 & 1 & 0 & -6.94500 & 0.87140 & 2.59710 \\
\hline 29 & 1 & 0 & -8.12130 & 2.10530 & 2.06170 \\
\hline 30 & 1 & 0 & -8.08500 & 0.50830 & 1.26120 \\
\hline 31 & 1 & 0 & -2.86300 & -2.26600 & -1.98010 \\
\hline 32 & 1 & 0 & -2.07440 & -0.75900 & -2.49730 \\
\hline 33 & 1 & 0 & -1.74940 & -2.12730 & 0.22980 \\
\hline 34 & 1 & 0 & -1.00830 & 0.59190 & -0.81180 \\
\hline 35 & 1 & 0 & -1.14710 & -0.52840 & 2.04320 \\
\hline 36 & 1 & 0 & -0.92810 & 1.17960 & 1.58220 \\
\hline 37 & 1 & 0 & -2.45360 & 0.34170 & 1.20570 \\
\hline 38 & 1 & 0 & 0.36540 & 2.98410 & -1.20090 \\
\hline 39 & 1 & 0 & 1.17030 & 5.19440 & -0.39740 \\
\hline 40 & 1 & 0 & 4.89700 & 3.41540 & 0.81670 \\
\hline 41 & 1 & 0 & 4.10180 & 1.19170 & 0.03510 \\
\hline 42 & 1 & 0 & 4.46800 & 6.09970 & 0.17500 \\
\hline 43 & 1 & 0 & 2.81450 & 6.56580 & 0.63040 \\
\hline 44 & 1 & 0 & 3.87520 & 5.74830 & 1.80120 \\
\hline 45 & 22 & 0 & 1.93340 & -1.75640 & 0.47540 \\
\hline 46 & 17 & 0 & 3.32270 & -0.92100 & 1.95430 \\
\hline 47 & 17 & 0 & 3.05010 & -3.48400 & -0.37530 \\
\hline 48 & 17 & 0 & 0.52860 & -2.72960 & 1.89330 \\
\hline 49 & 17 & 0 & -0.11740 & -2.54870 & -1.40670 \\
\hline
\end{tabular}

